\title{
Development of the Table of Initial Isolation and Protective Action Distances for the 2004 Emergency Response Guidebook
}

Decision and Information Sciences Division Argonne National Laboratory 



\title{
Development of the Table of Initial Isolation and Protective Action Distances for the 2004 Emergency Responce Guidebook
}

\author{
by \\ D.F.Brown \\ Decision and Information Sciences Division, Argonne National Laboratory \\ and \\ W.A. Freeman, R.A. Carhart, and M. Krumpolc \\ University of Chicago
}

May 2005

Work sponsored by U.S. Department of Transportation, Research and Special Programs Administration,

Office of Hazardous Materials Technology
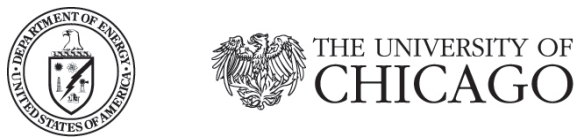

Argonne National Laboratory is managed by

The University of Chicago for the U.S. Department of Energy 


\begin{abstract}
About Argonne National Laboratory
Argonne is managed by The University of Chicago for the U.S. Department of Energy under contract W-31-109-Eng-38. The Laboratory's main facility is outside Chicago, at 9700 South Cass Avenue, Argonne, Illinois 60439. For information about Argonne and its pioneering science and technology programs, see www.anl.gov.
\end{abstract}

\title{
Availability of This Report
}

This report is available, at no cost, at http://www.osti.gov/bridge. It is also available on paper to U.S. Department of Energy and its contractors, for a processing fee, from:

U.S. Department of Energy

Office of Scientific and Technical Information

P.O. Box 62

Oak Ridge, TN 37831-0062

phone (865) 576-8401

fax (865) 576-5728

reports@adonis.osti.gov

\begin{abstract}
Disclaimer
This report was prepared as an account of work sponsored by an agency of the United States Government. Neither the United States Government nor any agency thereof, nor The University of Chicago, nor any of their employees or officers, makes any warranty, express or implied, or assumes any legal liability or responsibility for the accuracy, completeness, or usefulness of any information, apparatus, product, or process disclosed, or represents that its use would not infringe privately owned rights. Reference herein to any specific commercial product, process, or service by trade name, trademark, manufacturer, or otherwise, does not necessarily constitute or imply its endorsement, recommendation, or favoring by the United States Government or any agency thereof. The views and opinions of document authors expressed herein do not necessarily state or reflect those of the United States Government or any agency thereof, Argonne National Laboratory, or The University of Chicago.
\end{abstract}




\section{CONTENTS}

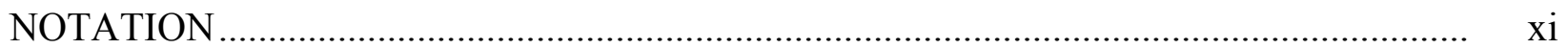

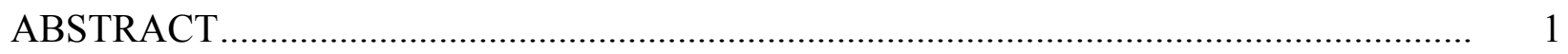

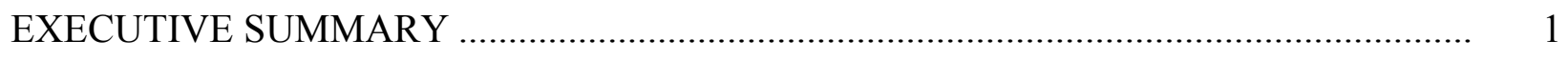

1 INTRODUCTION ...................................................................................... 5

1.1 Overview of the Emergency Response Guidebook .......................................... 5

1.2 Organization of this Report....................................................................... 7

2 GENERAL METHODOLOGY ....................................................................... 9

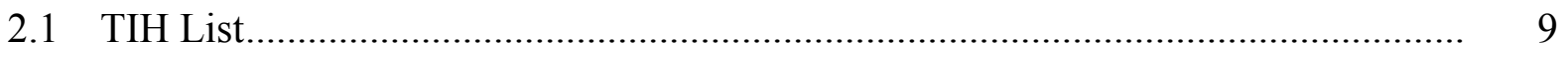

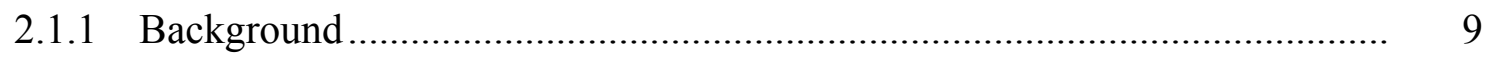

2.1.2 Changes in the TIH List for the ERG2004 ......................................... 11

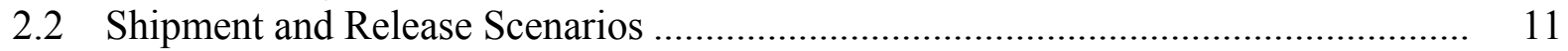

2.2.1 Shipment Profiles .......................................................................... 13

2.2.2 Treatment of Chemical Agents ........................................................... 14

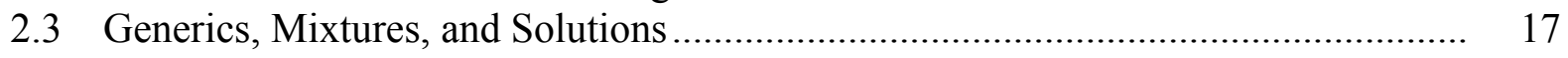

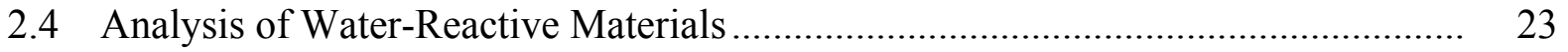

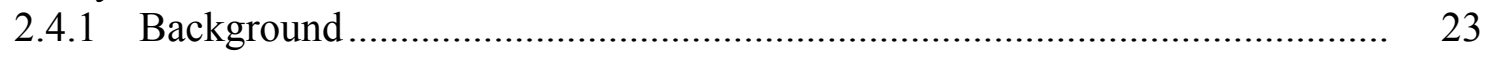

2.4.2 Identification and Evaluation of Candidates............................................ 24

2.4.3 Experimental Program ............................................................................ 25

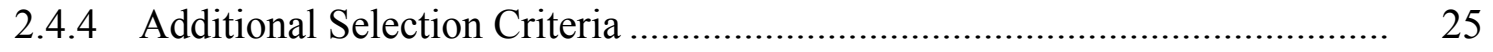

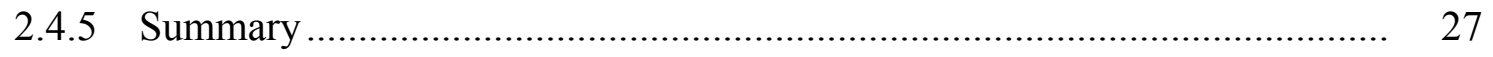

2.5 Determination of Initial Isolation Distances ................................................ 27

3 STATISTICAL ACCIDENT SCENARIO ANALYSIS AND CASRAM...................... 29

3.1 Statistical Scenario Analysis ........................................................................ 30

3.1.1 Overview of Analysis Steps............................................................... 30

3.1.2 The HMIS database...................................................................... 31

3.1.3 Geographic Incident Distributions ...................................................... 31

3.1.4 Temporal Incident Distributions ......................................................... 32

3.1.5 Discharge Fraction Distributions ........................................................... 32

3.2 Meteorological Database Used to Prepare the Guidebook .................................. 41

3.2.1 Meteorological and Site Data............................................................ 43

3.2.2 Meteorological Preprocessor ................................................................. 43

3.2.3 Water Temperature for TIHWR Reaction Rates .................................... 47

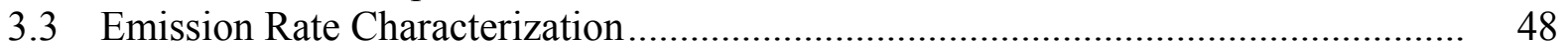

3.3.1 Overview of Release Types ............................................................... 48

3.3.2 CASRAM Emission Model and Its Application to the ERG2004 Analysis.. 50 


\section{CONTENTS (Cont.)}

3.4 Analysis of Atmospheric Dispersion .................................................................... 57

3.4.1 Overview of Atmospheric Dispersion ........................................................ 58

3.4.2 CASRAM Dispersion Model................................................................ 62

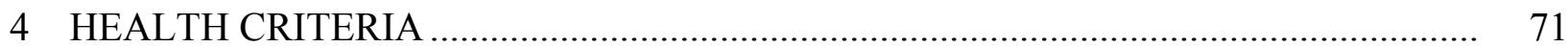

4.1 Review of Past Development Efforts.................................................................. 72

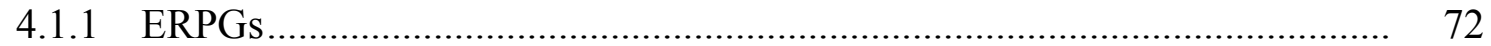

4.1.2 Comparisons of ERPGs with other Health Criteria ...................................... 72

4.1.3 Expert Panel Review............................................................................ 74

4.2 Procedure Used to Develop Health Criteria............................................................... 75

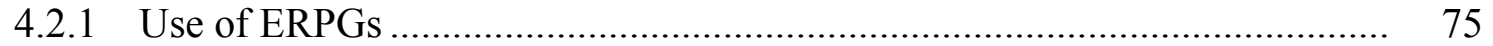

4.2.2 Use of Acute Inhalation Lethality Data in Animals..................................... 75

4.2.3 Use of Alternative Health Criteria .............................................................. 78

4.2.4 Summary ....................................................................................... 78

4.3 Comparison to Other Procedures ......................................................................... 79

4.4 Future Modifications........................................................................................ 80

4.4.1 Additions of New ERPG Values.......................................................... 80

4.4.2 Acute Exposure Guidance Levels ............................................................. 81

4.5 Glossary for Section 4 ............................................................................. 82

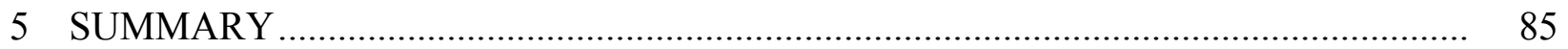

5.1 Safe Distance Distributions and Protective Action Distances ................................... 85

5.2 Presentation of the Table in the Guidebook ........................................................... 91

5.3 Extensions of the ERG Analysis........................................................................... 92

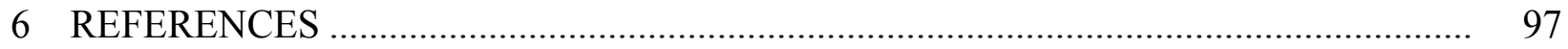

APPENDIX A Table of Initial Isolation and Protective Action Distances in the 2004 Emergency Response Guidebook ............................................ 103

APPENDIX B: Chemicals Analyzed for the ERG2004 …................................................. 127

APPENDIX C: Experimental Program Conducted to Support the Water Reactivity Analysis....................................................................... 135

APPENDIX D: Additional Details on Water-Reactive Materials ........................................ 193 


\section{FIGURES}

1.1 Illustration Showing How the ERG2004 Defines the Initial Isolation

and Protective Action Zones for Use by a First Responder....

2.1 Schematic of the Methodology Used to Prepare the ERG2004 Table

of Initial Isolation and Protective Action Distances

3.1 Hourly Distributions of Rail and Highway Transportation-Related Hazardous Material Releases Occurring During Accident-Related and En Route/Nonaccident Incidents from the HMIS Database, 1990-2002

3.2 Monthly Distributions of Rail and Highway Transportation-Related Hazardous Material Releases Occurring During Accident-Related and

En Route/Nonaccident Incidents from the HMIS Database, 1990-2002

3.3 Discharge Fraction Cumulative Probability for Bulk Nonpressurized and Low-Pressure Containers as Derived from the Analysis of the HMIS Database for the ERG2004 Analysis

3.4 Discharge Fraction Cumulative Probability for Bulk Pressurized Containers as Derived from the Analysis of the HMIS Database for the ERG2004 Analysis .......

3.5 Locations of the 105 National Weather Service Stations Used

for Statistical Meteorological Characterization

3.6 Water Quality Network Stations Used to Determine Variations

of Average Water Temperature by Julian Day and Location

3.7 Important Source Types for Inhalation Risk Considered in the ERG2004 Analysis.

3.8 Typical Daytime Convective Boundary Layer Showing an Instantaneous Wind Field and an Instantaneous Material Concentration Field from

a Ground-Level Release.

3.9 Typical Nighttime Stable Boundary Layer Showing an Instantaneous Wind Field and an Instantaneous Material Concentration Field from a Ground-Level Release.

5.1 Frequency of Safe Distances for Small Nighttime Chlorine Spills as Determined in the ERG2004 Analysis

5.2 Frequency of Safe Distances for Large Nighttime Chlorine Spills as Determined in the ERG2004 Analysis 


\section{FIGURES (Cont.)}

5.3 Frequency of Safe Distances for Small Daytime Chlorine Spills

as Determined in the ERG2004 Analysis

5.4 Frequency of Safe Distances for Large Daytime Chlorine Spills

as Determined in the ERG2004 Analysis

5.5 Frequency of Safe Distances for Small Nighttime Trichlorosilane Spills into Water as Determined in the ERG2004 Analysis

5.6 Frequency of Safe Distances for Large Nighttime Trichlorosilane Spills into Water as Determined in the ERG2004 Analysis

5.7 Frequency of Safe Distances for Small Daytime Trichlorosilane Spills into Water as Determined in the ERG2004 Analysis

5.8 Frequency of Safe Distances for Large Daytime Trichlorosilane Spills into Water as Determined in the ERG2004 Analysis

5.9 Frequency of Safe Distances for Large Daytime Chlorine Spills Occurring as a Result of Vehicular Accidents and Tank Car Derailments

5.10 Frequency of Safe Distances for Large Daytime Chlorine Spills for Different Wind Speed Ranges.

5.11 Frequency of Safe Distances for Highway Accidents Involving Fuming Nitric Acid 4,000-gal Cargo Tanks for Summer and Winter Conditions, Illustrating the Effects of Seasonal Climatology

5.12 Frequency of Safe Distances for a Chlorine Tank Car Release Greater than 1,000 gal, Occurring from 11 a.m. to 2 p.m. on a Sunny Day with the Wind Between 4 and $6 \mathrm{~m} / \mathrm{s}$, Showing the Effect on the Safe-distance Distribution as Each Constraint Is Imposed.

C.1 Apparatus Used to Measure the Amounts and Rates of Evolution of Gases When Substances React with Water

D.1 Example of a Simple First-Order Process from the Experimental Series Together with the Derived Parameters for Eq. D.1

D.2 Example of a First-Order Process from the Experimental Series where Dissolution of the TIH Product Competes with the Evolution 


\section{FIGURES (Cont.)}

D.3 Example of a First-Order Rate Process Competing with Dissolution

of the TIH Gas for which only Two Rising Points were Measured.

D.4 Example of a First-Order Rate Process Competing with Dissolution

of the TIH Gas for which only One Rising Point was Measured

D.5 Example of an Autocatalytic Reaction with an Initial By-Product

Greatly Reducing Availability of Water for Subsequent Reaction

\section{TABLES}

2.1 Ratio of En Route/Nonaccident Releases to Accident-Related Releases for Various Container Types as Derived from the HMIS Database for 1990-2002.

2.2 Mixed Rail and Highway Shipment Profile Data for Liquids

2.3 Mixed Rail and Highway Shipment Profile Data for Gases

2.4 Chemical Warfare Agents listed in the ERG2004 Table of Initial Isolation and Protective Action Distances

2.5 Chemicals Used to Determine Initial Isolation and Protective Action

Distances for the Generic Material Described as Liquefied Gas,

Poisonous, Flammable, n.o.s

2.6 Summary of Generic Compounds on DOT TIH List and Corresponding

Surrogates Employed for the ERG2004

2.7 Summary of Mixtures and Solutions on DOT TIH List and How They

Were Treated for the ERG2004

3.1 Geographic Distribution of Highway and Rail Transportation Incidents

Modeled in the ERG2004 Analysis, by Percentage

3.2 Coefficients for Calculating Discharge Fraction Cumulative Probability

Distributions for Accident-related Releases for a Variety of Containers.

3.3 Coefficients for Calculating Discharge Fraction Cumulative Probability

Distributions for En Route/Nonaccident Releases for a Variety of Containers. 


\section{TABLES (Cont.)}

3.4 Incident Counts Involving Drums in Accident-related Releases in the HMIS Database Shown with the Number of Which Involved Over $90 \%$ of the Total Shipment Amount Being Released

3.5 Incident Counts Involving Cylinders in Accident-related Releases in the HMIS Database Shown with the Number of Which Involved Over $90 \%$ of the Total Shipment Amount Being Released

3.6 Coefficients for Calculating Discharge Fraction Cumulative Probability Distributions in Eq. 3.3 for Accident-related Releases Involving Drums and Cylinders

3.7 Incident Counts Involving Drums In En Route/Nonaccident Releases in the HMIS Database Shown with the Number that Involved Only One Container and the Number with Over $90 \%$ of the Total Shipment Amount Being Released

3.8 Incident Counts Involving Cylinders in En Route/Nonaccident Releases in the HMIS Database Shown with the Number that Involved Only One Container and the Number with Over $90 \%$ of the Total Shipment Amount Being Released .......

3.9 Coefficients for Calculating Discharge Fraction Cumulative Probability Distributions in Eq. 3.3 for En Route/Nonaccident Releases Involving Drums and Cylinders

4.1 Order of Acute Lethality Data for Estimating Health Criteria ............................... 76

4.2 Summary of the Basis for Health Criteria Used to Prepare the ERG2004 ................ 79

4.3 Comparison of ERPG-2 Values with Final AEGL-2 Values for 8 TIH Materials and the \% Change in Large Spill Nighttime Distance if the AEGL-2 Value Replaced the ERPG-2 Value in the ERG Analysis

5.1 Safe Distances at Several Percentiles for Chlorine Releases as Determined in the ERG2004 Analysis.

5.2 Safe Distances at Several Percentiles for Trichlorosilane Releases into Water as Determined in the ERG2004 Analysis .

5.3 Safe Distances at Several Percentiles for Land-based Phosphorous

Trichloride Releases as Determined in the ERG2004 Analysis 


\section{TABLES (Cont.)}

5.4 Safe Distances at Several Percentiles for Ethylene Oxide Releases as Determined in the ERG2004 Analysis

A.1 Table of Initial Isolation and Protective Action Distances ................................... 106

B.1 Chemicals Analyzed for the ERG2004 ...................................................... 130

D.1 Supplementary Information on Water-Reactive Materials in the ERG2004 for Materials Modeled by a Simple First-order Process

D.2 Supplementary Information on Water-Reactive Materials in the ERG2004 for 5 Liquid Chlorosilanes Modeled Using an Initial Induction Period Followed by a Simple First-order Process

D.3 Chemicals Reported to Be Water-Reactive in the Scientific Literature 


\section{NOTATION}

The following is a list of the acronyms, initialisms, and abbreviations (including units of measure) used in this document.

\section{ACRONYMS AND ABBREVIATIONS}

$\begin{array}{ll}\text { ABL } & \text { atmospheric boundary layer } \\ \text { ACGIH } & \text { American Conference of Governmental Industrial Hygienists } \\ \text { AEGL } & \text { Acute Exposure Guidance Level (National Advisory Committee) } \\ \text { AIHA } & \text { American Industrial Hygiene Association } \\ \text { CAS } & \text { Chemical Abstracts Services } \\ \text { CASRAM } & \text { Chemical Accident Statistical Risk Assessment Model } \\ \text { CBL } & \text { convective boundary layer } \\ \text { CFR } & \text { Code of Federal Regulations } \\ \text { CWIC } & \text { crosswind-integrated concentration } \\ \text { DOT } & \text { U.S. Department of Transportation } \\ \text { EEGL } & \text { Emergency Exposure Guidance Level (National Research Council) } \\ \text { EEL } & \text { Emergency Exposure Level (AIHA Journal) } \\ \text { EPA } & \text { U.S. Environmental Protection Agency } \\ \text { ERG } & \text { Emergency Response Guidebook } \\ \text { ERPG } & \text { Emergency Response Planning Guideline (AIHA) } \\ \text { ERPG-1 } & \text { ERPG Level 1 } \\ \text { ERPG-2 } & \text { ERPG Level 2 } \\ \text { ERPG-3 } & \text { ERPG Level 3 } \\ \text { GDP } & \text { gross domestic product } \\ \text { GLB } & \text { Great Lakes Buoy } \\ \text { HCl } & \text { hydrogen chloride } \\ \text { HF } & \text { hydrogen fluoride } \\ \text { HMIS } & \text { Hazardous Materials Information System } \\ \text { HSE } & \text { Health and Safety Executive } \\ \text { IDLH } & \text { immediately dangerous to life and health (NIOSH level) } \\ \text { LC50 } & \text { median lethal concentration; concentration lethal to } \\ & \text { 50\% of an exposed population } \\ \text { LC } \text { LO } & \text { lowest reported lethal concentration } \\ \text { LOC } & \text { level of concern (EPA) } \\ \text { NIOSH } & \text { National Institute for Occupational Safety and Health } \\ \text { n.o.s. } & \text { not otherwise specified } \\ \text { OSHA } & \text { Occupational Safety and Health Administration } \\ \text { PAD } & \text { Protective Action Distance } \\ \text { PEL } & \text { permissible exposure limit (OSHA) } \\ \text { RD } 50 & \text { concentration producing a 50\% respiratory rate decrease in test animals; } \\ & \text { used to estimate severe respiratory irritation } \\ \text { SBL } & \text { stratified boundary layer } \\ \text { SEB } & \text { surface energy budget } \\ & \end{array}$


SEBMET Surface Energy Budget Meteorological (model)

SLOT specified level of toxicity (HSE)

STEL short-term exposure limit (ACGIH)

TIH toxic by inhalation

TIHWR toxic by inhalation by water reactivity

TLV threshold limit value (ACGIH)

TLV-C ceiling TLV

TLV-TWA time-weighted average TLV

UN United Nations

USGS U.S. Geological Survey

WQN Water Quality Network

\section{UNITS OF MEASURE}

$\begin{array}{ll}{ }^{\mathrm{atm}} \mathrm{C} & \text { atmosphere }\left(1.01 \times 10^{5} \mathrm{~Pa}\right) \\ \mathrm{cm} & \text { degree(s) Celsius } \\ \mathrm{cm} & \text { centimeter(s) } \\ \mathrm{ft} & \text { foot (feet) } \\ \mathrm{g} & \text { gram(s) } \\ \mathrm{gal} & \text { gallon(s) } \\ \mathrm{h} & \text { hour(s) } \\ \mathrm{K} & \text { degree(s) Kelvin } \\ \mathrm{kg} & \text { kilogram(s) } \\ \mathrm{kPa} & \text { kilopascal(s) } \\ \mathrm{m} & \text { meter(s) } \\ \mathrm{m} & \text { square meter(s) } \\ \mathrm{m} & \text { cubic meter(s) } \\ \mu \mathrm{m} & \text { micrometer(s) } \\ \mathrm{mi} & \text { mile(s) } \\ \mathrm{min} & \text { minute(s) } \\ \mathrm{mL} & \text { milliliter(s) } \\ \mathrm{mmol} & \text { millimole(s) } \\ \mathrm{Pa} & \text { Pascal(s) } \\ \mathrm{ppm} & \text { part(s) per million } \\ \mathrm{s} & \text { second(s) } \\ & \end{array}$




\title{
DEVELOPMENT OF THE TABLE OF INITIAL ISOLATION AND PROTECTIVE ACTION DISTANCES FOR THE 2004 EMERGENCY RESPONSE GUIDEBOOK
}

\author{
by \\ D.F. Brown, W.A. Freeman, ${ }^{*}$ R.A. Carhart, ${ }^{*}$ M. Krumpolc*
}

\begin{abstract}
This report provides technical documentation for values in the Table of Initial Isolation and Protective Action Distances (PADs) in the 2004 Emergency Response Guidebook (ERG2004). The objective for choosing the PADs specified in the ERG2004 is to balance the need to adequately protect the public from exposure to potentially harmful substances against the risks and expenses that could result from overreacting to a spill. To quantify this balance, a statistical approach is adopted, whereby the best available information is used to conduct an accident scenario analysis and develop a set of up to 1,000,000 hypothetical incidents. The set accounts for differences in containers types, incident types, accident severity (i.e., amounts released), locations, times of day, times of year, and meteorological conditions. Each scenario is analyzed using detailed emission rate and atmospheric dispersion models to calculate the downwind chemical concentrations from which a "safe distance" is determined. The safe distance is defined as the distance downwind from the source at which the chemical concentration falls below health protection criteria. The American Industrial Hygiene Association's Emergency Response Planning Guideline Level 2 (ERPG-2) or equivalent is the health criteria used. The statistical sample of safe distance values for all incidents considered in the analysis are separated into four categories: small spill/daytime release, small spill/nighttime release, large spill/daytime release, and large spill/nighttime release. The $90^{\text {th }}$-percentile safe distance values for each of these groups became the PADs that appear in the ERG2004.
\end{abstract}

\section{EXECUTIVE SUMMARY}

Chemical spills resulting from the transport of toxic materials can pose substantial hazards to the general public and to first responders. To address these hazards, first responders can consult the Emergency Response Guidebook (ERG), which helps them determine whether a spill is potentially hazardous and what actions to take. The 2004 edition of this guidebook, entitled 2004 Emergency Response Guidebook (i.e., ERG2004), was prepared by the

* University of Illinois at Chicago. 
U.S. Department of Transportation (DOT), Transport Canada, and the Secretariat of Transport and Communications of Mexico.

The ERG provides information on fire-fighting and mitigation strategies as well as on potential health effects associated with various chemicals, which it lists under their proper shipping names and four-digit United Nations (UN) identification numbers. The ERG also provides Initial Isolation Distances and Protective Action Distances (PADs) for a subset of the chemicals that are toxic by inhalation $(\mathrm{TIH})$ or that react with water to produce TIH gases (TIHWR). The Initial Isolation Distance identifies the radius of a zone around the release from which all people not directly involved in emergency response are to be kept away. The PAD is the downwind distance from the release that defines a zone in which persons should be either evacuated or sheltered in place, depending on the nature of the population at risk (e.g., density, age, health) and the severity of the incident.

This report provides the technical documentation for the values in the ERG2004 Table of Initial Isolation and Protective Action Distances (hereafter referred to as the Table). The objective for choosing the PADs specified in the ERG2004 was to balance the need to adequately protect the public from exposure to potentially harmful substances against the risks and expenses that could result from overreacting to a spill. To quantify this balance, a "level of protection" is defined. This level represents the probability that the listed PAD will allow sufficient protection of the public. A 90\% level of protection was selected for the ERG2004.

Quantitative analysis of the level of protection requires a statistical approach to specify the PAD. To achieve this objective, we use the best available information to conduct accident scenario analyses, resulting in a set of up to 1,000,000 hypothetical incidents for each material. The set accounts for differences in the container types, incident types, accident severities (i.e., amounts released), accident locations, times of day, times of year, and meteorological conditions involved. Each scenario is analyzed using detailed emission rate and atmospheric dispersion models to calculate the downwind chemical concentrations from which a "safe distance" is determined. The safe distance is defined as the distance downwind from the source at which the chemical concentration falls below the health protection criteria. The health criteria used are the American Industrial Hygiene Association's Emergency Response Planning Guideline Level 2 (ERPG-2) or equivalent. The ERPG-2 criteria denote the highest chemical concentration at which persons will suffer no irreversible or other serious health effects that could impair their ability to take protective action. The statistical sample of safe distance values for all incidents for each material is separated into four categories: small spill/daytime release, small spill/nighttime release, large spill/daytime release, and large spill/nighttime release. The $90^{\text {th }}$-percentile safe distance values for each of these groups are the PADs that appear in the Table.

This report presents the entire methodology used to produce the Table, much of which is unchanged from the ERG2000 and companion report. Specific improvements and changes in the methodology from the ERG2000 are noted below.

1. The experimental program for evaluating water reactive materials initiated for the ERG2000 were continued for the ERG2004. These experiments covered 35 materials in addition to the 21 evaluated for the ERG2000. As a result of 
the experiments, 14 new materials were added to the TIHWR list and 5 were deleted.

2. We reanalyzed the Hazardous Materials Information System (HMIS) database to incorporate accident records from recent years. The biggest change resulting from this reanalysis was development of a different method for treating shipments with multiple package freight containers (e.g., 25 cylinders, etc.) that is a more detailed and consistent system as compared to the previous approach. The overall result of this is a reduction of the predicted frequency of incidents where large numbers of package freight containers fail simultaneously. This had the net effect of causing the large spill distance estimates to increase slightly, since fewer package freight incidents had releases large enough to fall into the large spill category. Our new system for treating package freight also increased large spill distances for the Hazard Zone A gases. Changes in discharge fractions for bulk containers were fairly minor.

3. We developed several new shipment profiles for the ERG2004 tailored specifically to individual chemicals based on our analysis of HMIS and American Association of Railroads rail waybill data. Some adjustment was also made to existing profiles, most notably those for Hazard Zone A gases. (The shipment profiles specified the bulk and package freight containers typically used to transport the material, as well as the relative frequency at which each type of container is involved in incidents.) 


\section{INTRODUCTION}

Transportation of hazardous materials creates numerous opportunities for the release of toxic substances into the environment due to traffic accidents, train derailments, equipment failures, and human error. Such releases can pose serious acute hazards to the general public and to emergency response personnel who are the first to arrive at the scene. To help first responders determine whether a shipment is potentially hazardous and decide what actions should be taken if a toxic spill does occur, the Emergency Response Guidebook (ERG) is published by the U.S. Department of Transportation (DOT), Transport Canada, and Secretariat of Transport and Communications of Mexico. The most recent version is the 2004 edition of the ERG (DOT et al. 2004), titled 2004 Emergency Response Guidebook (hereafter referred to as ERG2004). The ERG provides essential information on fire-fighting, spill response, and potential public health effects. For chemicals that are toxic by inhalation (TIH) and chemicals that produce TIH gases upon reaction with water (TIHWR), the ERG provides Initial Isolation Distances and Protective Action Distances. The Initial Isolation Distance defines the radius of the zone around the spill that should be accessed solely by people who are directly involved in emergency response. The protective action distance (PAD) is the distance downwind from the source of the release to which persons should be either evacuated or sheltered-in-place, depending on the severity of the incident and the nature of the population (e.g., density, age, health).

This report was prepared to document the methodology used to prepare the ERG2004 Table of Initial Isolation and Protective Action Distances (hereafter referred to as "the Table"). The PADs in the Table were calculated to balance the need to adequately protect the public from exposure to potentially harmful substances against the risks and expenses that could result from overreacting to a spill. In determining the PADs, this balance was quantified in terms of a level of protection; namely, the probability that the listed PAD will allow sufficient protection of the public. The level of protection adopted for the ERG was $90 \%$. Clearly, a quantitative analysis of the level of protection requires a statistical approach, the underlying technical basis of which is described in this report.

\subsection{OVERVIEW OF THE EMERGENCY RESPONSE GUIDEBOOK}

The ERG is designed for use by first responders to determine the appropriate level of action during the initial stages of a hazardous materials transportation incident. Although knowledgeable in the field of law enforcement and public protection, the first responder is usually not an expert on hazardous materials. Thus, the ERG provides a compact source of essential information on which to base reasonable decisions under often difficult conditions. 
As noted, for TIH materials the ERG provides the Table of Initial Isolation and Protective Action Distances. Figure 1.1 illustrates the basic information presented in the Table and its application. To properly use the Table, a responder must first determine the following:

- United Nations (UN) identification number and/or proper shipping name of the material being transported,

- Direction of the prevailing wind,

- Whether the spill is small (60 gal or less) or large (more than 60 gal),

- Whether it is day or night, and

- Any special conditions that could preclude the use of the values given in the Table (e.g., releases of multiple tank cars, topographical anomalies, etc.).

As depicted in Figure 1.1, releases of TIH materials result in downwind concentrations of vapor that decrease with distance from the release point (assuming that the release is not buoyant). At some downwind distance, the concentration decreases to a level below which no protective action is necessary. This distance is the Protective Action Distance (PAD). The Protective Action Zone (PAZ) is defined as a square region having a side dimension equal to the PAD and lying downwind and symmetrically about the accident location. Note that since the PAZ lies downwind of the source, the first responder must first ascertain the wind direction to correctly use the information in the ERG. Since wind direction knowledge is inherent in the PAZ definition, the PAZ does not need to be circular.

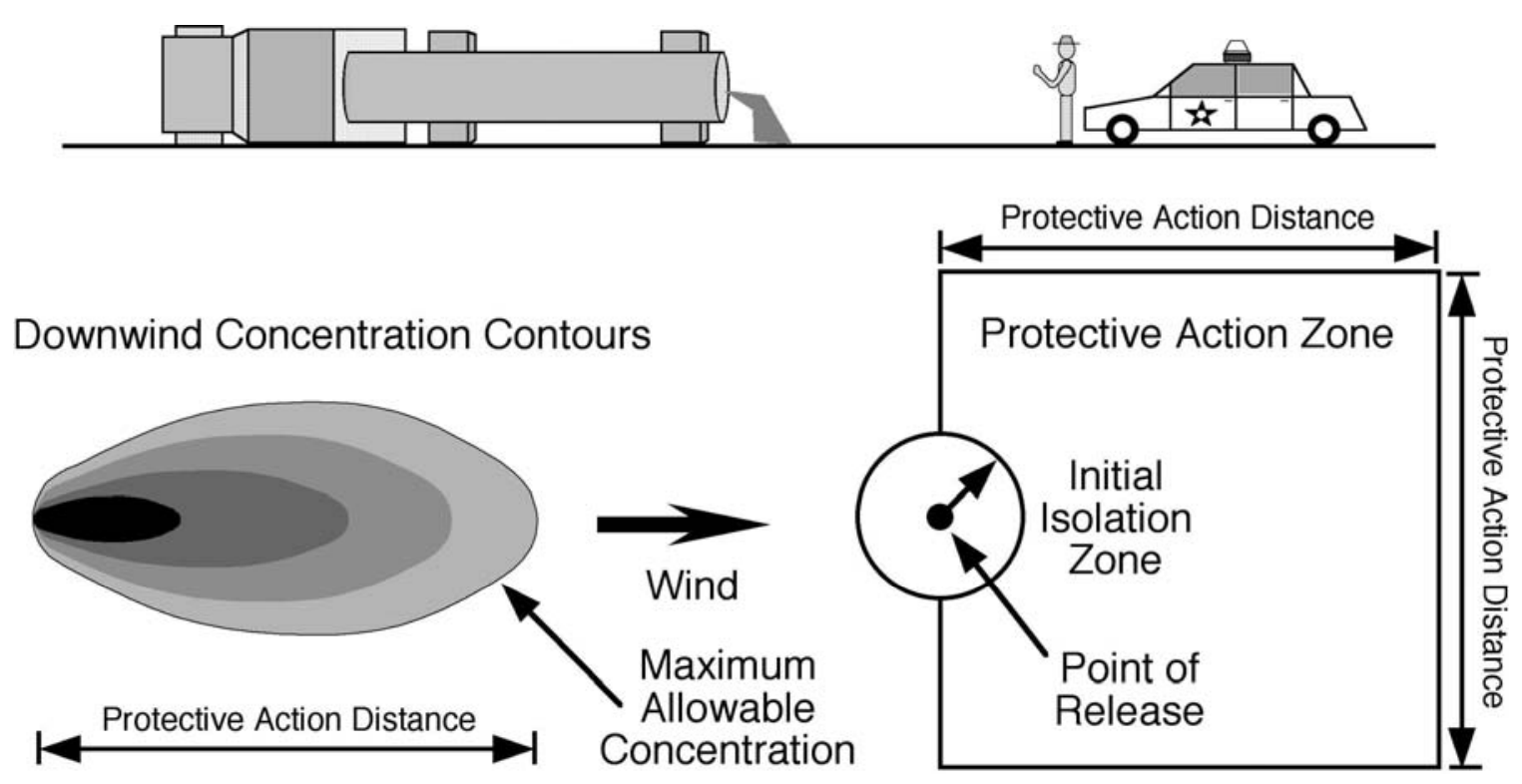

FIGURE 1.1 Illustration Showing How the ERG2004 Defines the Initial Isolation and Protective Action Zones for Use by a First Responder 
The Initial Isolation Distance specifies a circular zone surrounding the accident site. Persons not involved with the response should be kept clear of this zone. Persons in the Initial Isolation Zone may be exposed to potentially life-threatening health effects downwind of the accident site and dangerous concentrations upwind due to wind direction variability. Specification of the Initial Isolation Distances is treated in a method analogous to that for the PADs.

The range of cases for which the ERG could be used is extreme, covering everything from a minor leak from a compressed gas cylinder to a catastrophically ruptured tank car. However, the space requirements necessary to address a wide range of cases for all the TIH materials in the ERG would be prohibitive. To narrow the range of cases for which a particular PAD is employed and keep the number entries listed in the ERG reasonable, four PAD values are provided for each material that address (1) whether the incident involves a small spill or a large spill and (2) whether it occurs during the day or night. Here, a small spill is defined as the spillage of a single drum or cylinder, or a small leak from a bulk container corresponding to the limits defined previously. Whether the spill occurs during the day or night is very important in considering downwind dispersion of the released chemical as discussed in Section 3. Of course, a multitude of other weather and spill variables such as wind speed, cloud cover, and time (apart from just day or night) can greatly affect the necessary PAD for a specific incident. For this reason, we have adopted a statistical approach to determine the percentage of time a PAD will be sufficient in actual accidents.

\subsection{ORGANIZATION OF THIS REPORT}

Section 2 presents a detailed overview of the methodology used to calculate the Initial Isolation Distances and PADs. Included in this discussion is an examination of issues related to the TIH list; treatment of generic compounds, mixtures, and solutions; treatment of chemical warfare agents; and treatment of water-reactive materials. Section 3 provides details on the statistical scenario analysis applied to materials in the Table, as well as technical details on the consequence models used. Section 4 documents the health criteria, or threshold chemical concentrations, used to specify the Initial Isolation and PADs. Section 5 discusses the safe distance distributions developed as a result of the analysis and describes how the PADs were determined from these distributions. Appendix A presents the Table (DOT et al. 2004). Appendixes B-D contain chemical data and details of the experiments conducted to identify and quantify $\mathrm{TIH}$ gas emission rates from water-reactive materials. 


\section{GENERAL METHODOLOGY}

The methodology used in preparing the Table for the ERG2004 is illustrated schematically in Figure 2.1. The starting point for the analysis is the list of TIH materials developed by DOT and Transport Canada (see discussion in Section 2.1). This list contains a few additions to and deletions from the ERG2000 list, with most additions being water reactive materials. For each material in the list, the authorized mode of shipping, as outlined in the Code of Federal Regulations (CFR), is combined with commodity flow information and historical incident data to develop a shipment profile. Shipment profiles, which are discussed in Section 2.2.1, are used in the analysis to determine the types of transportation incidents that could occur for particular materials or classes of materials.

The shipment profiles are then used to conduct a statistical analysis of accident scenarios. The result of this analysis is a set of up to 1,000,000 hypothetical incidents based on the best available statistical information, which takes into account variability in container type, incident type, accident severity (i.e., release amount), location, time of day, time of year, and meteorology. Several of the important release parameters are selected from statistical distributions from transportation-related hazardous materials releases cataloged in the Hazardous Materials Information System (HMIS) database maintained by the DOT Research and Special Programs Administration.

Each scenario is then analyzed using detailed emission rate and atmospheric dispersion models to calculate an airborne chemical concentration footprint. The safe distance for a specific scenario is then chosen as the greatest downwind distance where the concentration exceeds the health criteria for the chemical involved in the incident. The health criteria, which are exposuretime-dependent threshold concentrations, are based on American Industrial Hygiene Association (AIHA) Emergency Response Planning Guideline Level 2 (ERPG-2) or an equivalent guideline as detailed in Section 4. The safe distance estimates for the entire set of hypothetical incidents considered in the analysis provide a distribution of safe distances that correspond to the many transportation-related releases that could occur. Incidents are then categorized by time (day, night) and spill size ( 60 gal or less $=$ small, more than 60 gal $=$ large $)$. The PADs appearing in the ERG2004 are then selected as the $90^{\text {th }}$ percentile values for these individual categories. The Initial Isolation Zones are calculated in a similar manner on the basis of health criteria for lifethreatening effects.

\subsection{TIH LIST}

\subsubsection{Background}

For the purposes of our analysis, we classify materials on this TIH list into several different categories as follows: (1) pure chemicals, (2) mixtures, (3) solutions, and (4) generics (e.g., UN 1953: liquefied gas, toxic, flammable, n.o.s.). As is explained later, generic 


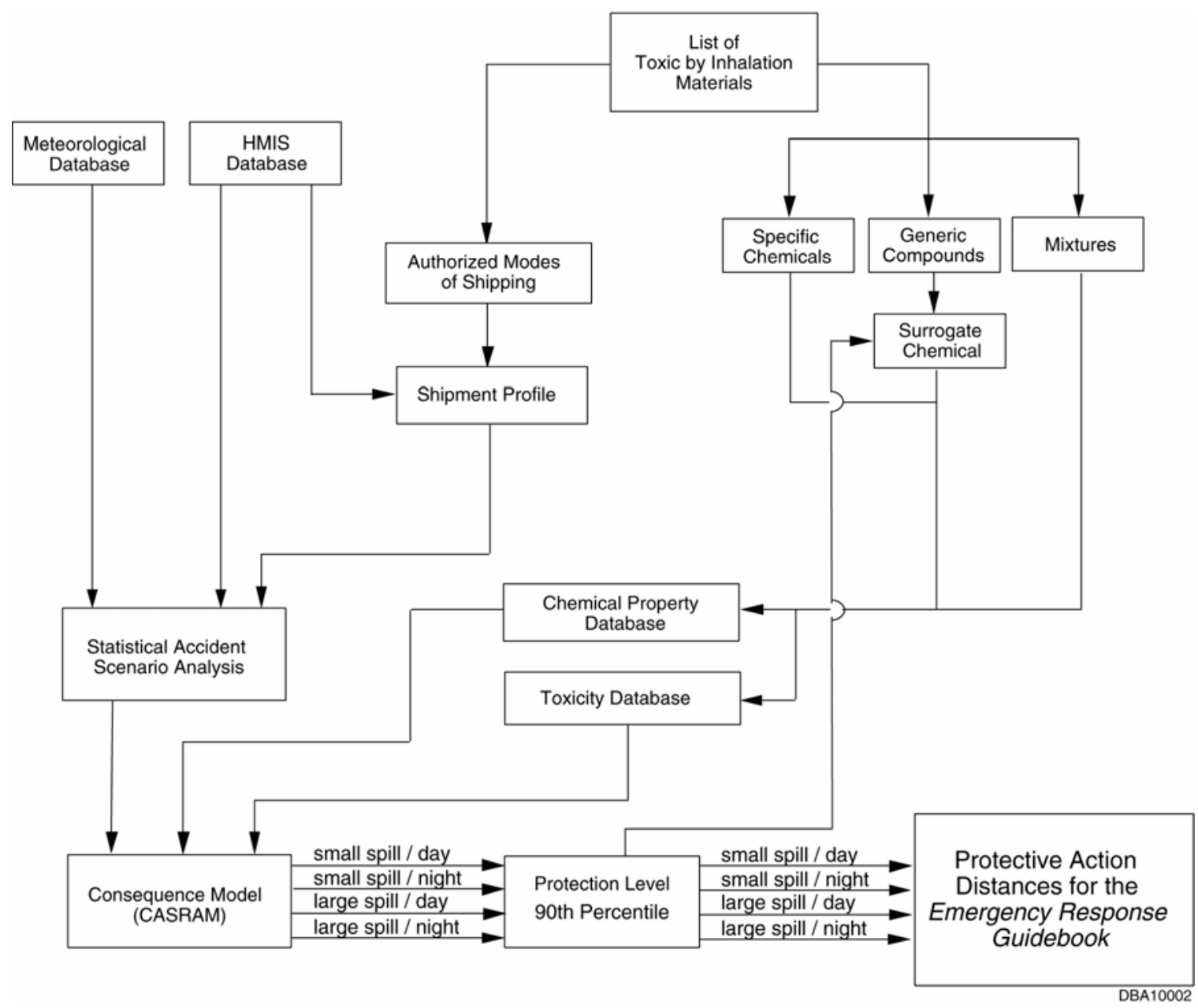

FIGURE 2.1 Schematic of the Methodology Used to Prepare the ERG2004 Table of Initial Isolation and Protective Action Distances

compounds are further divided into subcategories on the basis of Hazard Zone designation, as defined in Title 49, Part 173, Section 133 of the CFR (49 CFR 173.133). TIH materials fall into 4 Hazard Zones for gases (A, B, C, and D) and 2 for liquids (A and B). The ERG2004 lists PADs for 156 single compounds, 15 mixtures, and 26 generics. In addition, 70 water-reactive materials, defined here as materials that emit a TIH gas upon contact with water, are also included in the $\mathrm{TIH}$ list. Of the 70 water-reactive materials in the Table, several are also TIH compounds that produce a secondary, sometimes more toxic TIH gas upon reaction with water.

Each category is handled individually. Single chemicals are specified according to a Chemical Abstracts Services (CAS) number, which is a unique numerical identifier for each chemical compound. A unique identifier is necessary to avoid problems with chemical name synonyms. It is important to note that the UN number is not a unique identifier, in that two or more chemicals may be associated with a particular UN number. One example is UN 1076, which is used for both phosgene (CAS 75-44-5) and diphosgene (503-38-8), materials with structural similarities but markedly different physical properties. Mixtures are treated by 
considering the individual components in the mixture. Generics are modeled by using a surrogate compound, with the surrogate being the worst case of the materials considered for the particular generic description and hazard zone. Mixtures and generics are discussed in Section 2.3. Waterreactive materials are treated in a manner similar to that for regular TIH materials with modifications to the physical models for determining source emission rate. The treatment of water reactivity is discussed in Section 2.4 and Appendixes C and D.

\subsubsection{Changes in the TIH List for the ERG2004}

In contrast to the numerous changes in the TIH list that occurred between 1996 and 2000 , there were relatively few substantive changes made in the TIH list for the 2004 Table, with the exception of (1) the addition of several new TIHWR materials and (2) the removal of all nerve and blister warfare agents that are Hazard Zone A and B liquids ${ }^{1}$ for transportation-related releases. Considering water-reactive materials, 14 new materials were added to the TIHWR list, and 5 materials were removed. The additions and removals were based on experimental studies conducted for the ERG2004 analysis, which built on the experimental program initiated for the ERG2000. The treatment of water-reactive materials is discussed in depth in Section 2.4 and Appendixes $\mathrm{C}$ and $\mathrm{D}$. The nerve and blister agents were removed because these materials do not appear in 49 CFR 172.101 (Hazardous Materials Table). Note that all these materials were retained for weapons-related releases, as discussed in Section 2.2.2. Wording revisions and synonym entry additions and deletions resulted in the addition of about 25 entries and removal of 6 , thus bringing the names in the Table into agreement with current UN naming conventions.

For future editions of the ERG, we recommend deletion of coal gas (UN 1023) and oil gas (UN 1071). These are officially TIH materials; however, our analysis of the mixture constituents for these materials indicate the median lethal concentration $\left(\mathrm{LC}_{50}\right)$ is well above $10,000 \mathrm{ppm}$. We also recommend the addition of acrylonitrile (UN 1093) and methylamine (UN 1061). Acrylonitrile is a liquid with a vapor pressure of $11.9 \mathrm{kPa}$ at $20^{\circ} \mathrm{C}$, so in order to fit the criteria as a Hazard Zone B liquid, the $\mathrm{LC}_{50}$ would need to be below 1,000 ppm per 49 CFR 173.333. Several references cited in development of National Institute for Occupational Safety and Health (NIOSH) immediately dangerous to life and health (IDLH) values indicate the 1-h LC 50 may be below 1,000 ppm (see www.cdc.gov/niosh/idlh). Methylamine is a gas. The reference used to set the IDLH value indicates the $1-\mathrm{h} \mathrm{LC}_{50}$ is below 5,000 ppm, the necessary criterion to become a Hazard Zone D gas. For reference, if acrylonitrile and methylamine were included in the Table, the large night spill protective action distances for them would be 0.37 and $1.45 \mathrm{mi}$, respectively.

\subsection{SHIPMENT AND RELEASE SCENARIOS}

To specify a level of protection as defined previously, we constructed a set of representative accident scenarios for each material on the TIH list. The first step in this process

1 Prior to this decision, Tris-(2-chloroethyl) amine (the warfare agent HN-3; UN 2810) was removed since investigation of the vapor pressure and $\mathrm{LC}_{50}$ values for this material showed it to be a Hazard Zone $\mathrm{C}$ liquid. 
was to segregate the total transportation of the particular $\mathrm{TIH}$ material into a set of discrete shipments using shipment profiles. Shipment profiles specify bulk and package freight containers typically used in transporting the material as well as the relative frequency that each container is involved in an incident. The goal of this analysis was to assign each chemical a set of representative shipments that reflect its transportation in the United States, Canada, and Mexico. Specification of shipment profiles is discussed in Section 2.2.1. These shipment profiles were used with the Chemical Accident Statistical Risk Assessment Model (CASRAM) to simulate tens of thousands of accidents for each chemical in a fashion similar to that used by Brown et al. (2001).

For each shipment, analyses are conducted for two types of releases: those occurring (1) during a traffic accident or a train derailment and (2) while en route from the origin to the destination but not during an accident or derailment. These release types are referred to as accident-related and en route/nonaccident releases, respectively. The latter category includes releases occurring as a result of cargo shifts, valve failures, corrosion-induced container failure, etc. Such releases are much more common than accident-related releases, as shown by the HMIS incident data for many container types (several of which are not used to transport $\mathrm{TIH}$ materials) summarized in Table 2.1. However, most en route/nonaccident releases are minor.

A special case within the shipment profile concept is the treatment of chemical warfare agents. These materials were first considered in the ERG2000. In 2000, entries were given for two release scenarios: (1) transportation-related release scenarios and (2) weapons-related release scenarios whereby the material is released in a criminal or terrorist act. However, the transportation-related release scenario was removed in 2004 as discussed in Section 2.1. The treatment of chemical warfare agents is outlined in Section 2.2.2.

TABLE 2.1 Ratio of En Route/Nonaccident Releases to Accident-Related Releases for Various Container Types as Derived from the HMIS Database for 1990-2002

\begin{tabular}{lc}
\hline Container Type & $\begin{array}{c}\text { Ratio of En Route/Nonaccident } \\
\text { Releases to Accident-Related } \\
\text { Releases }\end{array}$ \\
\hline 111AW tank car & 13 \\
112JW and 105A tank car & $23^{\mathrm{a}}$ \\
MC306 cargo tank & 0.2 \\
MC307 cargo tank & 2.2 \\
MC312 cargo tank & 3.3 \\
MC330/331 cargo tank & 0.5 \\
Small and medium drums & 20 \\
Large drums and portable tanks & 7 \\
Package freight cylinders & 5 \\
\hline & \\
a For the ERG2004 analysis, the ratio for 111A tank cars is used
\end{tabular}




\subsubsection{Shipment Profiles}

The starting point for the development of shipment profiles is 49 CFR 172.101 and associated subsections that specify the authorized shipping modes and packaging for hazardous materials. These specifications substantially influence the amount of material that could be released in a transport-related accident and other important factors that govern the release hazard, such as the relative frequencies of each container type being involved in a release and the discharge fractions resulting from releases (the amount of material released in an incident relative to the container capacity). For example, most Division 2.3 gases under Hazard Zone A cannot be transported in bulk form. Consequently, such materials are shipped only in package freight containers (drums, cylinders). The resultant total shipping volumes are thus much less than those associated with a typical bulk shipment even though there can be many package freight containers in a single shipment.

The shipment profiles for most chemicals fall into one of the following nine general classes:

1. Gases dominated by rail transportation,

2. Gases with mixed rail and highway transportation,

3. Gases (Hazard Zone B) dominated by highway transportation,

4. Liquids dominated by rail transportation,

5. Liquids with mixed rail and highway transportation,

6. Liquids dominated by highway transportation (unused),

7. Bulk-forbidden gases and liquids authorized under 49 CFR 173.192,

8. Bulk-forbidden gases and liquids authorized under 49 CFR 173.302, and

9. Organophosphates authorized under 49 CFR 173.334.

Chemicals with a 49 CFR 173.245 authorization for bulk transportation are included in the bulk-forbidden classes. For these chemicals (and the ones for which bulk is forbidden), the PADs for large spills were estimated from shipments containing up to 25 cylinders or drums. A release from this number of package freight containers is equivalent to a release from a small bulk cargo tank (e.g., 1,500 kg). Larger containers were not included in this analysis because it was believed that they would add an unrealistic degree of conservatism to the results, since the probability of these materials being shipped in true bulk form appears to be very low.

Generally, a shipment was designated as being either rail- or highway-dominated on the basis of available commodity flow data (these data are for several widely shipped chemicals) and a survey of incidents from the HMIS database. If no information other than data on packing 
authorizations within 49 CFR was known and if the material was authorized for bulk transport, the mixed rail and highway shipment profile was used.

For 2004, we developed several new shipment profiles tailored specifically to several high-volume chemicals, such as chlorine and ammonia, based on our analysis of HMIS, rail waybill data from the American Association of Railroads, and other commodity flow information together with 49 CFR information. In addition, we created several additional profiles for materials called out for specific treatment in 49 CFR, such as diborane, fluorine, methyl mercaptin, and tetranitromethane. Some adjustment was made to existing profiles, most notably those for Hazard Zone A gases based on discussions with Compressed Gas Association members and analysis of HMIS incidents.

Examples of mixed rail and highway shipment profiles are provided in Tables 2.2 and 2.3 for liquids and gases, respectively. Three classes of shipments are listed for each profile: bulk rail transportation, bulk highway transportation, and package freight transportation. To provide some perspective on the relative influence of the shipment classes and release types on releases modeled in the ERG2004 analysis, the percentage of total releases represented by each type is listed for all releases, releases of 5-60 gal, and releases of more than 60 gal. When all releases are considered, en route/nonaccident incidents make up the majority of releases modeled for the shipment profiles given in Tables 2.2 and 2.3. However, in considering releases of more than 60 gal (i.e., "large spills" in the Table), accident-related incidents make up the majority of cases. For such releases, package freight incidents were the most common and bulk highway incidents the least. Because PADs are set by the $90^{\text {th }}$-percentile value, incidents involving bulk containers had a far greater influence on PAD values than did incidents involving package freight containers, since bulk containers usually involved larger release amounts.

\subsubsection{Treatment of Chemical Agents}

The 1995 Tokyo subway sarin attack and the events on and following September 11, 2001, have made the first-response community more aware of the threat from the malicious use of chemical and biological agents. For this reason, the ERG includes Initial Isolation Distances and PADs for various chemical agents that could be used as weapons. In addition, a separate section entitled Criminal/Terrorist Use of Chemical/Biological/Radiological Agents (page 354 of the ERG2004) provides information on identification, response, and decontamination strategies for personnel who must respond to a suspected release of such materials.

Table 2.4 lists 26 chemical compounds for which Initial Isolation and Protective Action Distances were calculated for cases in which they would be used as a weapon. (The table shows 36 chemical warfare agent names for the 26 compounds.) Accordingly, entries in the ERG2004 Table for these materials include the statement "when used as a weapon." Several of these materials are also industrial chemicals that appear separately in ERG2004 as transportationrelated releases. Entries for weapons-related use of chemical agents, however, are listed under the common or military name for the compound, not the chemical name. For example, for 
TABLE 2.2 Mixed Rail and Highway Shipment Profile Data for Liquids ${ }^{a}$

\begin{tabular}{|c|c|c|c|c|c|c|c|}
\hline \multirow[b]{2}{*}{ Shipment } & \multirow[b]{2}{*}{$\begin{array}{l}\text { Transport } \\
\text { Mode }\end{array}$} & \multirow[b]{2}{*}{ Container } & \multirow[b]{2}{*}{$\begin{array}{l}\text { Shipment } \\
\text { Amount }\end{array}$} & \multirow[b]{2}{*}{$\begin{array}{c}\text { Release } \\
\text { Type }\end{array}$} & \multicolumn{3}{|c|}{$\begin{array}{l}\text { Percent of Total Releases by } \\
\text { Type, Listed by Release Amount }\end{array}$} \\
\hline & & & & & $\begin{array}{c}\text { All } \\
\text { Releases }\end{array}$ & $5-60 \mathrm{gal}^{\mathrm{b}}$ & $>60 \mathrm{gal}^{\mathrm{b}}$ \\
\hline 1 & Rail & $\begin{array}{l}\text { DOT Class } \\
112 \text { tank car }\end{array}$ & $80,000 \mathrm{~kg}$ & $\begin{array}{l}\mathrm{A} \\
\mathrm{E}\end{array}$ & $\begin{array}{r}3.2 \\
41.6\end{array}$ & $\begin{array}{r}1.1 \\
23.0\end{array}$ & $\begin{array}{l}24.9 \\
21.7\end{array}$ \\
\hline 2 & Highway & $\begin{array}{l}\text { DOT } 312 \\
\text { cargo tank }\end{array}$ & $20,000 \mathrm{~kg}$ & $\begin{array}{l}\mathrm{A} \\
\mathrm{E}\end{array}$ & $\begin{array}{l}1.6 \\
5.0\end{array}$ & $\begin{array}{l}0.7 \\
6.4\end{array}$ & $\begin{array}{r}13.6 \\
6.2\end{array}$ \\
\hline 3 & Highway & $\begin{array}{l}\text { Ten } 55 \text {-gal } \\
5 \mathrm{C} \text { drums }\end{array}$ & $550 \mathrm{gal}$ & $\begin{array}{l}\mathrm{A} \\
\mathrm{E}\end{array}$ & $\begin{array}{r}2.3 \\
46.2 \\
\end{array}$ & $\begin{array}{r}3.0 \\
65.8 \\
\end{array}$ & $\begin{array}{l}14.0 \\
19.6\end{array}$ \\
\hline
\end{tabular}

a This profile covers three shipment classes and two release types, accident-related (A) and en route/nonaccident (E). Percentages are provided for the total number of incidents that occurred in the various shipment classes and release types. Percentages are given for all releases, releases of 5$60 \mathrm{gal}$, and releases of more than $60 \mathrm{gal}$.

b Data provided for methyl hydrazine (UN 1244). Other materials with this profile would have similar results.

TABLE 2.3 Mixed Rail and Highway Shipment Profile Data for Gases ${ }^{a}$

\begin{tabular}{|c|c|c|c|c|c|c|c|}
\hline \multirow[b]{2}{*}{ Shipment } & \multirow[b]{2}{*}{$\begin{array}{l}\text { Transport } \\
\text { Mode }\end{array}$} & \multirow[b]{2}{*}{ Container } & \multirow[b]{2}{*}{$\begin{array}{c}\text { Shipment } \\
\text { Amount }\end{array}$} & \multirow{2}{*}{$\begin{array}{l}\text { Re- } \\
\text { lease } \\
\text { Type }\end{array}$} & \multicolumn{3}{|c|}{$\begin{array}{l}\text { Percent of Total Releases by } \\
\text { Type, Listed by Release Amount }\end{array}$} \\
\hline & & & & & $\begin{array}{c}\text { All } \\
\text { Releases } \\
\end{array}$ & $5-60 \mathrm{gal}^{\mathrm{b}}$ & $>60 \mathrm{gal}^{\mathrm{b}}$ \\
\hline 1 & Rail & $\begin{array}{l}\text { DOT Class } 105, \\
112 \text { tank car }\end{array}$ & $80,000 \mathrm{~kg}$ & $\begin{array}{l}\mathrm{A} \\
\mathrm{E}\end{array}$ & $\begin{array}{r}2.5 \\
56.5\end{array}$ & $\begin{array}{r}0.8 \\
13.6\end{array}$ & $\begin{array}{l}27.9 \\
18.2\end{array}$ \\
\hline 2 & Highway & $\begin{array}{l}\text { MC331 cargo } \\
\text { tank }\end{array}$ & $20,000 \mathrm{~kg}$ & $\begin{array}{l}\mathrm{A} \\
\mathrm{E}\end{array}$ & $\begin{array}{l}1.2 \\
0.6\end{array}$ & $\begin{array}{l}1.2 \\
0.5\end{array}$ & $\begin{array}{l}9.9 \\
3.7\end{array}$ \\
\hline 3 & Highway & $\begin{array}{l}\text { Fifteen } 19 \text {-gal } 3 \mathrm{~A} \\
\text { or } 4 \mathrm{~A} \text { cylinders }\end{array}$ & $285 \mathrm{gal}$ & $\begin{array}{l}\mathrm{A} \\
\mathrm{E}\end{array}$ & $\begin{array}{r}4.9 \\
34.4\end{array}$ & $\begin{array}{r}9.0 \\
74.9\end{array}$ & $\begin{array}{l}27.4 \\
13.0\end{array}$ \\
\hline
\end{tabular}

a This profile covers three shipment classes and two release types, accident-related (A) and en route/nonaccident (E). Percentages are provided for the total number of incidents that occurred in the various shipment classes and release types. Percentages are given for all releases, releases of 5$60 \mathrm{gal}$, and releases of more than $60 \mathrm{gal}$.

b Data provided for chlorine trifluoride (UN 1749). Other materials with this profile would have similar results. 
TABLE 2.4 Chemical Warfare Agents listed in the ERG2004 Table of Initial Isolation and Protective Action Distances

\begin{tabular}{|c|c|c|c|c|c|}
\hline $\begin{array}{l}\text { UN } \\
\text { No. }\end{array}$ & $\begin{array}{c}\text { Chemical Warfare } \\
\text { Agent Name }\end{array}$ & Chemical Compound Name & $\begin{array}{c}\text { Small } \\
(\mathrm{kg})\end{array}$ & $\begin{array}{l}\text { Large } \\
(\mathrm{kg})\end{array}$ & Type $^{\mathrm{a}}$ \\
\hline 1051 & $\mathrm{AC}$ & Hydrogen cyanide & 60 & 30,000 & $\mathrm{SH}$ \\
\hline 1076 & DP & Diphosgene & 30 & 500 & $\mathrm{AL}$ \\
\hline 1076 & CG & Phosgene & 60 & 1,500 & $\mathrm{SH}$ \\
\hline 1556 & MD & Methyldichloroarsine & 30 & 500 & $\mathrm{AL}$ \\
\hline 1556 & PD & Phenyldichloroarsine & 30 & 500 & AL \\
\hline 1589 & $\mathrm{CK}$ & Cyanogen chloride & 30 & 500 & $\mathrm{AL}$ \\
\hline 1694 & $\mathrm{CA}$ & Bromobenzyl cyanides & 10 & 500 & AS \\
\hline 1697 & $\mathrm{CN}$ & Chloroacetophenone & 10 & 500 & AS \\
\hline 1698 & DM & \multirow{2}{*}{$\begin{array}{l}\text { Diphenylaminechloroarsine (10-Chloro-5,10- } \\
\text { dihydrophenarsazine) }\end{array}$} & \multirow{2}{*}{10} & \multirow{2}{*}{500} & \multirow{2}{*}{ AS } \\
\hline 1698 & Adamsite & & & & \\
\hline 1699 & DA & Diphenylchloroarsine & 10 & 500 & AS \\
\hline 1892 & ED & Ethyldichloroarsine & 30 & 500 & $\mathrm{AL}$ \\
\hline 2188 & SA & Arsine & 60 & 1,500 & $\mathrm{SH}$ \\
\hline 2810 & $\mathrm{H}$ & \multirow{3}{*}{ Bis-(2-chloroethyl) sulfide } & \multirow{3}{*}{2} & \multirow{3}{*}{100} & \multirow{3}{*}{ SP } \\
\hline 2810 & HD & & & & \\
\hline 2810 & Mustard & & & & \\
\hline 2810 & $\mathrm{HN}-1$ & Bis-(2-chloroethyl) ethylamine & 2 & 100 & SP \\
\hline 2810 & $\mathrm{HN}-2$ & Bis-(2-chloroethyl) methylamine & 2 & 100 & $\mathrm{SP}$ \\
\hline 2810 & $\mathrm{HN}-3$ & Tris-(2-chloroethyl) amine & 2 & 100 & $\mathrm{SP}$ \\
\hline 2810 & $\mathrm{~L}$ & \multirow{4}{*}{ Dichloro-(2-chlorovinyl) arsine } & \multirow{4}{*}{2} & \multirow{4}{*}{100} & \multirow{4}{*}{ SP } \\
\hline 2810 & Lewisite & & & & \\
\hline 2810 & $\mathrm{HL}^{\mathrm{b}}$ & & & & \\
\hline 2810 & Mustard lewisite ${ }^{b}$ & & & & \\
\hline 2810 & $\mathrm{BZ}$ & \multirow{2}{*}{ 3-Quinuclidinyl benzilate } & \multirow{2}{*}{10} & \multirow{2}{*}{500} & \multirow{2}{*}{ AS } \\
\hline 2810 & Buzz & & & & \\
\hline 2810 & $\mathrm{CS}$ & o-Chlorobenzylidene malononitrile & 10 & 500 & AS \\
\hline 2810 & $\mathrm{DC}$ & Diphenylcyanoarsine & 10 & 500 & AS \\
\hline 2810 & GA & \multirow{2}{*}{ Ethyl N,N-dimethylphosphoramidocyanidate } & \multirow{2}{*}{2} & \multirow{2}{*}{100} & \multirow{2}{*}{ SP } \\
\hline 2810 & Tabun & & & & \\
\hline 2810 & GB & \multirow{2}{*}{ Isopropyl methylphosphonofluoridate } & \multirow{2}{*}{2} & \multirow{2}{*}{100} & \multirow{2}{*}{ SP } \\
\hline 2810 & Sarin & & & & \\
\hline 2810 & GD & \multirow{2}{*}{ Pinacolyl methylphosphonofluoridate } & \multirow{2}{*}{2} & \multirow{2}{*}{100} & \multirow{2}{*}{ SP } \\
\hline 2810 & Soman & & & & \\
\hline 2810 & GF & Cyclohexyl methylphosphonofluoridate & 2 & 100 & $\mathrm{SP}$ \\
\hline 2810 & VX & $\begin{array}{l}\text { O-Ethyl S-(2-diisopropylaminoethyl) } \\
\text { methylphosphonothiolate }\end{array}$ & 2 & 100 & SP \\
\hline 2811 & $\mathrm{CX}$ & Phosgene oxime & 10 & 500 & AS \\
\hline
\end{tabular}

a $\mathrm{SH}=$ releases by shipment sabotage; $\mathrm{AL}=$ aerosolized liquid; $\mathrm{AS}=$ aerosolized solid; $\mathrm{SP}=$ spray or explosive release.

b Because of uncertainties in defining the composition, $\mathrm{HL}$ and mustard lewisite were treated as standard lewisite (L). 
weapons-related entries, the first compound in Table 2.4 is listed as AC, whereas for transportation-related entries, this same compound is listed as hydrogen cyanide (UN 1051). These two terms refer to the same compound but with very different release scenarios.

The release scenarios for weapons-related incidents differ from those for transportationrelated incidents because they involve deliberate releases. Two maximum release sizes were used for each material considered in the analysis. In the statistical analysis, release amounts were uniformly distributed between 50 and $100 \%$ of these maximum release amounts. Various release types were modeled, depending on the material being released. The release types are denoted in Table 2.4 by two-letter codes. The codes AL and AS refer to aerosolized liquid and aerosolized solid release mechanisms, respectively. It is assumed that AL and AS would be dispersed in aerosolized form with a 2 - to $5-\mu \mathrm{m}$ particle size. This release mechanism is used for solid materials or for materials that have very slow evaporation rates; thus, aerosolization is the only way to disseminate them effectively. The code SP refers to a spray or explosive release. This release mechanism is considered the most likely one to be used to disperse nerve and blister agents, since they are typically thick liquids not readily amenable to direct aerosolization. In the scenario used in this analysis, the spray quickly settles on the ground to a depth up to $0.25 \mathrm{~mm}$ and then evaporates. The evaporation rate for these materials is limited by their low vapor pressures. The code $\mathrm{SH}$ refers to releases by shipment sabotage. This release mechanism is used for volatile TIH materials. For example, the large release scenario for hydrogen cyanide involves the sabotage of a large bulk container such as a small rail car. Small release amounts for these materials correspond to the release of a standard gas cylinder.

\subsection{GENERICS, MIXTURES, AND SOLUTIONS}

The Table lists a variety of compounds that are generic in nature. Two examples are liquefied gas, flammable, poisonous, n.o.s. (Inhalation Hazard Zone B) (UN 3160) and toxic liquid, corrosive, organic, n.o.s. (UN 2927). (The abbreviation n.o.s. stands for not otherwise specified.) Each generic compound can represent many independent chemicals that fit that description but are not individually listed in the ERG2004 or the Table. In the ERG2004, Initial Isolation and Protective Action Distances for generic compounds are based on the worst-case compound from the pool of chemicals in the overall analysis that matches that generic description. As an example of this process, the generic compound described as liquefied gas, flammable, poisonous, n.o.s. (Inhalation Hazard Zone B) (UN 1953), which is also listed in the Table as liquefied gas, toxic, flammable, n.o.s. (Inhalation Hazard Zone B) (UN 3160), is considered below. The six chemicals analyzed for the ERG2004 that are included in this category are listed in Table 2.5. Phosphorus pentafluoride (UN 2198) yields the maximum distances for large spills, whereas dichlorosilane (UN 2189) yields the maximum distances for small spills (shown in bold in Table 2.5). Also provided are eight entries for this generic category that reflect the variations in wording in the Table. These entries are rounded up to the nearest $0.1 \mathrm{mi} \mathrm{(100} \mathrm{ft} \mathrm{for} \mathrm{Initial} \mathrm{Isolation} \mathrm{Zones)} \mathrm{to} \mathrm{reflect} \mathrm{their} \mathrm{appearance} \mathrm{in} \mathrm{the} \mathrm{Table.}$

Table 2.6 lists all the generic compounds included in the Table and provides the subset of

chemicals from which their distances were calculated. Synonyms are not listed in Table 2.6, so 
TABLE 2.5 Chemicals Used to Determine Initial Isolation and Protective Action Distances for the Generic Material Described as Liquefied Gas, Poisonous, Flammable, n.o.s. (Inhalation Hazard Zone B) (UN 1953, UN 3160) ${ }^{a}$

\begin{tabular}{|c|c|c|c|c|c|c|c|}
\hline \multirow[b]{3}{*}{ UN No. } & \multirow[b]{3}{*}{ Name of Material } & \multicolumn{3}{|c|}{ Small Spills } & \multicolumn{3}{|c|}{ Large Spills } \\
\hline & & \multirow{2}{*}{$\begin{array}{l}\text { First Isolate } \\
\text { in All } \\
\text { Directions } \\
(\mathrm{ft}) \\
\end{array}$} & \multicolumn{2}{|c|}{$\begin{array}{l}\text { Then Protect } \\
\text { Persons } \\
\text { Downwind } \\
\text { during } \\
\end{array}$} & \multirow{2}{*}{$\begin{array}{l}\text { First Isolate } \\
\text { in All } \\
\text { Directions } \\
(\mathrm{ft}) \\
\end{array}$} & \multicolumn{2}{|c|}{$\begin{array}{l}\text { Then Protect } \\
\text { Persons } \\
\text { Downwind } \\
\text { during } \\
\end{array}$} \\
\hline & & & $\begin{array}{l}\text { Day } \\
(\mathrm{mi})\end{array}$ & $\begin{array}{c}\text { Night } \\
(\mathrm{mi})\end{array}$ & & $\begin{array}{l}\text { Day } \\
(\mathrm{mi})\end{array}$ & $\begin{array}{c}\text { Night } \\
(\mathrm{mi})\end{array}$ \\
\hline \multicolumn{8}{|c|}{ Potential Surrogate Compounds } \\
\hline 1026 & Cyanogen & 58 & 0.11 & 0.71 & 348 & 0.66 & 2.63 \\
\hline 1053 & Hydrogen sulfide & 16 & 0.03 & 0.17 & 681 & 1.29 & 3.84 \\
\hline 2189 & Dichlorosilane & 53 & 0.1 & 0.56 & 1,309 & 2.48 & 6.66 \\
\hline 2192 & Germane & 42 & 0.08 & 0.58 & 232 & 0.44 & 1.86 \\
\hline 2198 & Phosphorus pentafluoride & 79 & 0.15 & 0.99 & 507 & 0.96 & 2.84 \\
\hline 2204 & Carbonyl sulfide & 32 & 0.06 & 0.32 & 977 & 1.85 & 4.99 \\
\hline \multicolumn{8}{|c|}{ Synonymous Entries for Generic Compound Categories } \\
\hline 1953 & $\begin{array}{l}\text { Compressed gas, flammable, } \\
\text { poisonous, n.o.s. (Inhalation } \\
\text { Hazard Zone B) }\end{array}$ & 100 & 0.2 & 0.8 & 1,400 & 2.5 & 6.7 \\
\hline 1953 & $\begin{array}{l}\text { Compressed gas, flammable, toxic, } \\
\text { n.o.s. (Inhalation Hazard Zone B) }\end{array}$ & 100 & 0.2 & 0.8 & 1,400 & 2.5 & 6.7 \\
\hline 1953 & $\begin{array}{l}\text { Compressed gas, poisonous, } \\
\text { flammable, n.o.s. (Inhalation } \\
\text { Hazard Zone B) }\end{array}$ & 100 & 0.2 & 0.8 & 1,400 & 2.5 & 6.7 \\
\hline 1953 & $\begin{array}{l}\text { Compressed gas, toxic, flammable, } \\
\text { n.o.s. (Inhalation Hazard Zone B) }\end{array}$ & 100 & 0.2 & 0.8 & 1,400 & 2.5 & 6.7 \\
\hline 1953 & $\begin{array}{l}\text { Liquefied gas, flammable, } \\
\text { poisonous, n.o.s. (Inhalation } \\
\text { Hazard Zone B) }\end{array}$ & 100 & 0.2 & 0.8 & 1,400 & 2.5 & 6.7 \\
\hline 1953 & $\begin{array}{l}\text { Liquefied gas, flammable, toxic, } \\
\text { n.o.s. (Inhalation Hazard Zone B) }\end{array}$ & 100 & 0.2 & 0.8 & 1,400 & 2.5 & 6.7 \\
\hline 3160 & $\begin{array}{l}\text { Liquefied gas, poisonous, } \\
\text { flammable, n.o.s. (Inhalation } \\
\text { Hazard Zone B) }\end{array}$ & 100 & 0.2 & 0.8 & 1,400 & 2.5 & 6.7 \\
\hline 3160 & $\begin{array}{l}\text { Liquefied gas, toxic, flammable, } \\
\text { n.o.s. (Inhalation Hazard Zone B) }\end{array}$ & 100 & 0.2 & 0.8 & 1,400 & 2.5 & 6.7 \\
\hline
\end{tabular}

a This table provides distance estimates for all applicable entries in the ERG2004 Table of Initial Isolation and Protective Action Distances (DOT et al. 2004). The worst-case (maximum) distances for small spills and large spills for each column are shown in bold. 
TABLE 2.6 Summary of Generic Compounds on DOT TIH List and Corresponding Surrogates Employed for the ERG2004. Note that a different surrogate material may by used for each release category.

\begin{tabular}{|c|c|c|}
\hline $\begin{array}{c}\text { UN } \\
\text { Number }\end{array}$ & Proper Shipping Name & Surrogate \\
\hline 1953 & Liquefied gas, poisonous, flammable, n.o.s. & Worst cases among all TIH, flammable gases \\
\hline 1953 & Liquefied gas, poisonous, flammable, n.o.s. (Inhalation Hazard Zone A) & Worst cases among all TIH, flammable Hazard Zone A gases \\
\hline 1953 & Liquefied gas, poisonous, flammable, n.o.s. (Inhalation Hazard Zone B) & Worst cases among all TIH, flammable Hazard Zone B gases \\
\hline 1953 & Liquefied gas, poisonous, flammable, n.o.s. (Inhalation Hazard Zone C) & Worst cases among all TIH, flammable Hazard Zone C gases \\
\hline 1953 & Liquefied gas, poisonous, flammable, n.o.s. (Inhalation Hazard Zone D) & Worst cases among all TIH, Hazard Zone D gases \\
\hline 1955 & Liquefied gas, poisonous, n.o.s. & Worst cases among all TIH gases \\
\hline 1955 & Liquefied gas, poisonous, n.o.s. (Inhalation Hazard Zone A) & Worst cases among all TIH, Hazard Zone A gases \\
\hline 1955 & Liquefied gas, poisonous, n.o.s. (Inhalation Hazard Zone B) & Worst cases among all TIH, Hazard Zone B gases \\
\hline 1955 & Liquefied gas, poisonous, n.o.s. (Inhalation Hazard Zone C) & Worst cases among all TIH, Hazard Zone C gases \\
\hline 1955 & Liquefied gas, poisonous, n.o.s. (Inhalation Hazard Zone D) & Worst cases among all TIH, Hazard Zone D gases \\
\hline 1955 & Organic phosphate mixed with compressed gas & Parathion and compressed gas mixture \\
\hline 1967 & Insecticide gas, poisonous, n.o.s. & Parathion and compressed gas mixture \\
\hline 2810 & Poisonous liquid, n.o.s. & Worst cases among all TIH liquids \\
\hline 2810 & Poisonous liquid, n.o.s. (Inhalation Hazard Zone A) & Worst cases among all TIH Hazard Zone A liquids \\
\hline 2810 & Poisonous liquid, n.o.s. (Inhalation Hazard Zone B) & Worst cases among all TIH Hazard Zone B liquids \\
\hline 2810 & Poisonous liquid, organic, n.o.s. & Worst cases among all TIH organic liquids \\
\hline 2810 & Poisonous liquid, organic, n.o.s. (Inhalation Hazard Zone A) & Worst cases among all TIH, organic, Hazard Zone A liquids \\
\hline 2810 & Poisonous liquid, organic, n.o.s. (Inhalation Hazard Zone B) & Worst cases among all TIH, organic, Hazard Zone B liquids \\
\hline 2927 & Poisonous liquid, corrosive, n.o.s. & Worst cases among all TIH corrosive liquids \\
\hline 2927 & Poisonous liquid, corrosive, n.o.s. (Inhalation Hazard Zone A) & Worst cases among all TIH, corrosive, Hazard Zone A liquids \\
\hline 2927 & Poisonous liquid, corrosive, n.o.s. (Inhalation Hazard Zone B) & Worst cases among all TIH, corrosive, Hazard Zone B liquids \\
\hline 2927 & Toxic liquid, corrosive, organic, n.o.s. & Worst cases among all TIH corrosive, organic liquids \\
\hline 2927 & Toxic liquid, corrosive, organic, n.o.s. (Inhalation Hazard Zone A) & Worst cases among all TIH, corrosive, organic Hazard Zone A liquids \\
\hline 2927 & Toxic liquid, corrosive, organic, n.o.s. (Inhalation Hazard Zone B) & Worst cases among all TIH, corrosive, organic, Hazard Zone B liquids \\
\hline 2929 & Poisonous liquid, flammable, n.o.s. & Worst cases among all TIH flammable liquids \\
\hline 2929 & Poisonous liquid, flammable, n.o.s. (Inhalation Hazard Zone A) & Worst cases among all TIH, flammable, Hazard Zone A liquids \\
\hline 2929 & Poisonous liquid, flammable, n.o.s. (Inhalation Hazard Zone B) & Worst cases among all TIH, flammable, Hazard Zone B liquids \\
\hline 2929 & Poisonous liquid, flammable, organic, n.o.s. & Worst cases among all TIH flammable, organic, liquids \\
\hline 2929 & Poisonous liquid, flammable, organic, n.o.s. (Inhalation Hazard Zone A) & Worst cases among all TIH, flammable, organic, Hazard Zone A liquids \\
\hline 2929 & Poisonous liquid, flammable, organic, n.o.s. (Inhalation Hazard Zone B) & Worst cases among all TIH, flammable, organic, Hazard Zone B liquids \\
\hline 3122 & Poisonous liquid, oxidizing, n.o.s. & Worst cases among all TIH, flammable, liquids \\
\hline 3122 & Poisonous liquids, oxidizing, n.o.s. (Inhalation Hazard Zone A) & Worst cases among all TIH, flammable, Hazard Zone A liquids \\
\hline 3122 & Poisonous liquids, oxidizing, n.o.s. (Inhalation Hazard Zone B) & Worst cases among all TIH, oxidizing, Hazard Zone B liquids \\
\hline 3123 & Poisonous liquid, water-reactive, n.o.s. & Worst cases among all TIH liquids \\
\hline 3123 & Poisonous liquid, water-reactive, n.o.s. (Inhalation Hazard Zone A) & Worst cases among all TIH Hazard Zone A liquids \\
\hline 3123 & Poisonous liquid, water-reactive, n.o.s. (Inhalation Hazard Zone B) & Worst cases among all TIH Hazard Zone B liquids \\
\hline 3123 & Poisonous liquid, which in contact with water emits flammable gases, n.o.s. & Worst cases among all TIH flammable liquids \\
\hline
\end{tabular}


TABLE 2.6 (Cont.)

$\mathrm{UN}$

Number

Proper Shipping Name

Surrogate

3123 Poisonous liquid, which in contact with water emits flammable gases, n.o.s (Inhalation Hazard Zone A)

3123 Poisonous liquid, which in contact with water emits flammable gases, n.o.s. (Inhalation Hazard Zone B)

3160 Liquefied gas, poisonous, flammable, n.o.s.

3160 Liquefied gas, poisonous, flammable, n.o.s. (Inhalation Hazard Zone A)

3160 Liquefied gas, poisonous, flammable, n.o.s. (Inhalation Hazard Zone B)

3160 Liquefied gas, poisonous, flammable, n.o.s. (Inhalation Hazard Zone C)

3160 Liquefied gas, poisonous, flammable, n.o.s. (Inhalation Hazard Zone D)

3162 Liquefied gas, poisonous, n.o.s.

3162 Liquefied gas, poisonous, n.o.s. (Inhalation Hazard Zone A)

3162 Liquefied gas, poisonous, n.o.s. (Inhalation Hazard Zone B)

3162 Liquefied gas, poisonous, n.o.s. (Inhalation Hazard Zone C)

3162 Liquefied gas, poisonous, n.o.s. (Inhalation Hazard Zone D)

3275 Nitriles, poisonous, flammable, n.o.s.

$3276 \quad$ Nitriles, poisonous, n.o.s.

$3278 \quad$ Organophosphorus compound, poisonous, n.o.s.

3279 Organophosphorus compound, poisonous, flammable, n.o.s.

$3280 \quad$ Organoarsenic compound, n.o.s.

$3281 \quad$ Metal carbonyls, n.o.s.

3287 Poisonous liquid, inorganic, n.o.s.

3287 Poisonous liquid, inorganic, n.o.s. (Inhalation Hazard Zone A)

3287 Poisonous liquid, inorganic, n.o.s. (Inhalation Hazard Zone B)

3289 Poisonous liquid, corrosive, inorganic, n.o.s.

3289 Poisonous liquid, corrosive, inorganic, n.o.s. (Inhalation Hazard Zone A)

3289 Poisonous liquid, corrosive, inorganic, n.o.s. (Inhalation Hazard Zone B)

3303 Compressed gas, poisonous, oxidizing, n.o.s.

3303 Compressed gas, poisonous, oxidizing, n.o.s. (Inhalation Hazard Zone A)

3303 Compressed gas, poisonous, oxidizing, n.o.s. (Inhalation Hazard Zone B)

3303 Compressed gas, poisonous, oxidizing, n.o.s. (Inhalation Hazard Zone C)

3303 Compressed gas, poisonous, oxidizing, n.o.s. (Inhalation Hazard Zone D)

3304 Compressed gas, poisonous, corrosive, n.o.s.

3304 Compressed gas, poisonous, corrosive, n.o.s. (Inhalation Hazard Zone A)

3304

3304

3305

3305

(

Compressed gas, poisonous, corrosive, n.o.s. (Inhalation Hazard Zone C)

Compressed gas, poisonous, corrosive, n.o.s. (Inhalation Hazard Zone D)

Compressed gas, poisonous, flammable, corrosive, n.o.s.

Compressed gas, poisonous, flammable, corrosive, n.o.s. (Inh. Hazard Zone A)
Worst cases among all TIH, flammable, Hazard Zone A liquids

Worst cases among all TIH, flammable, Hazard Zone B liquids

Worst cases among all $\mathrm{TIH}$, flammable gases

Worst cases among all TIH, flammable Hazard Zone A gases

Worst cases among all TIH, flammable Hazard Zone B gases

Worst cases among all TIH, flammable Hazard Zone C gases

Worst cases among all TIH, flammable Hazard Zone D gases

Worst cases among all TIH gases

Worst cases among all TIH Hazard Zone A gases

Worst cases among all TIH Hazard Zone B gases

Worst cases among all TIH Hazard Zone C gases

Worst cases among all TIH Hazard Zone D gases

Methacrylonitrile

Methacrylonitrile

Methyl phosphonous dichloride

Methyl phosphonous dichloride

Tert-butylarsine

Nickel carbonyl

Worst cases among all TIH inorganic liquids

Worst cases among all TIH, inorganic, Hazard Zone A liquids

Worst cases among all TIH, inorganic, Hazard Zone B liquids

Worst cases among all $\mathrm{TIH}$, corrosive, inorganic liquids

Worst cases among all TIH, corrosive, inorganic, Hazard Zone A liquids

Worst cases among all TIH, corrosive, inorganic, Hazard Zone B liquids

Worst cases among all $\mathrm{TIH}$, oxidizing gases

Worst cases among all TIH, Hazard Zone A, oxidizing gases

Worst cases among all TIH, Hazard Zone B, oxidizing gases

Worst cases among all TIH, Hazard Zone C gases

Worst cases among all TIH, Hazard Zone D gases

Worst cases among all TIH, corrosive gases

Worst cases among all TIH, corrosive, Hazard Zone A gases

Worst cases among all TIH, corrosive, Hazard Zone B gases

Worst cases among all TIH, corrosive, Hazard Zone C gases

Worst cases among all TIH, corrosive, Hazard Zone D gases

Worst cases among all $\mathrm{TIH}$, corrosive gases

Worst cases among all TIH, corrosive, Hazard Zone A gases 
TABLE 2.6 (Cont.)

\begin{tabular}{|c|c|c|}
\hline $\begin{array}{c}\text { UN } \\
\text { Number } \\
\end{array}$ & Proper Shipping Name & Surrogate \\
\hline 3305 & Compressed gas, poisonous, flammable, corrosive, n.o.s. (Inh. Hazard Zone B) & Worst cases among all TIH, corrosive, Hazard Zone B gases \\
\hline 3305 & Compressed gas, poisonous, flammable, corrosive, n.o.s. (Inh. Hazard Zone C) & Worst cases among all TIH, corrosive, Hazard Zone C gases \\
\hline 3305 & Compressed gas, poisonous, flammable, corrosive, n.o.s. (Inh. Hazard Zone D) & Worst cases among all TIH, corrosive, Hazard Zone D gases \\
\hline 3306 & Compressed gas, poisonous, oxidizing, corrosive, n.o.s. & Worst cases among all $\mathrm{TIH}$, oxidizing, corrosive gases \\
\hline 3306 & Compressed gas, poisonous, oxidizing, corrosive, n.o.s. (Inh. Hazard Zone A) & Worst cases among all TIH, oxidizing, corrosive, Hazard Zone A gases \\
\hline 3306 & Compressed gas, poisonous, oxidizing, corrosive, n.o.s. (Inh. Hazard Zone B) & Worst cases among all TIH, oxidizing, corrosive, Hazard Zone B gases \\
\hline 3306 & Compressed gas, poisonous, oxidizing, corrosive, n.o.s. (Inh. Hazard Zone C) & Worst cases among all $\mathrm{TIH}$, corrosive, Hazard Zone $\mathrm{C}$ gases \\
\hline 3306 & Compressed gas, poisonous, oxidizing, corrosive, n.o.s. (Inh. Hazard Zone D) & Worst cases among all TIH, corrosive, Hazard Zone D gases \\
\hline 3307 & Liquefied gas, poisonous, oxidizing, n.o.s. & Worst cases among all $\mathrm{TIH}$, oxidizing gases \\
\hline 3307 & Liquefied gas, poisonous, oxidizing, n.o.s. (Inhalation Hazard Zone A) & Worst cases among all TIH, Hazard Zone A, oxidizing gases \\
\hline 3307 & Liquefied gas, poisonous, oxidizing, n.o.s. (Inhalation Hazard Zone B) & Worst cases among all TIH, Hazard Zone B, oxidizing gases \\
\hline 3307 & Liquefied gas, poisonous, oxidizing, n.o.s. (Inhalation Hazard Zone C) & Worst cases among all $\mathrm{TIH}$, Hazard Zone $\mathrm{C}$, oxidizing gases \\
\hline 3307 & Liquefied gas, poisonous, oxidizing, n.o.s. (Inhalation Hazard Zone D) & Worst cases among all TIH, Hazard Zone D, oxidizing gases \\
\hline 3308 & Liquefied gas, poisonous, corrosive, n.o.s. & Worst cases among all $\mathrm{TIH}$, corrosive gases \\
\hline 3308 & Liquefied gas, poisonous, corrosive, n.o.s. (Inhalation Hazard Zone A) & Worst cases among all TIH, corrosive, Hazard Zone A gases \\
\hline 3308 & Liquefied gas, poisonous, corrosive, n.o.s. (Inhalation Hazard Zone B) & Worst cases among all TIH, corrosive, Hazard Zone B gases \\
\hline 3308 & Liquefied gas, poisonous, corrosive, n.o.s. (Inhalation Hazard Zone C) & Worst cases among all TIH, corrosive, Hazard Zone C gases \\
\hline 3308 & Liquefied gas, poisonous, corrosive, n.o.s. (Inhalation Hazard Zone D) & Worst cases among all TIH, corrosive, Hazard Zone D gases \\
\hline 3309 & Liquefied gas, poisonous, flammable, corrosive, n.o.s. & Worst cases among all $\mathrm{TIH}$, corrosive gases \\
\hline 3309 & Liquefied gas, poisonous, flammable, corrosive, n.o.s. (Inhalation Hazard Zone A) & Worst cases among all TIH, corrosive, Hazard Zone A gases \\
\hline 3309 & Liquefied gas, poisonous, flammable, corrosive, n.o.s. (Inhalation Hazard Zone B) & Worst cases among all TIH, corrosive, Hazard Zone B gases \\
\hline 3309 & Liquefied gas, poisonous, flammable, corrosive, n.o.s. (Inhalation Hazard Zone C) & Worst cases among all $\mathrm{TIH}$, corrosive, Hazard Zone C gases \\
\hline 3309 & Liquefied gas, poisonous, flammable, corrosive, n.o.s. (Inhalation Hazard Zone D) & Worst cases among all TIH, corrosive, Hazard Zone D gases \\
\hline 3310 & Liquefied gas, poisonous, oxidizing, corrosive, n.o.s. & Worst cases among all $\mathrm{TIH}$, oxidizing, corrosive gases \\
\hline 3310 & Liquefied gas, poisonous, oxidizing, corrosive, n.o.s. (Inhalation Hazard Zone A) & Worst cases among all TIH, oxidizing, corrosive, Hazard Zone A gases \\
\hline 3310 & Liquefied gas, poisonous, oxidizing, corrosive, n.o.s. (Inhalation Hazard Zone B) & Worst cases among all TIH, oxidizing, corrosive, Hazard Zone B gases \\
\hline 3310 & Liquefied gas, poisonous, oxidizing, corrosive, n.o.s. (Inhalation Hazard Zone C) & Worst cases among all TIH, corrosive, Hazard Zone $\mathrm{C}$ gases \\
\hline 3310 & Liquefied gas, poisonous, oxidizing, corrosive, n.o.s. (Inhalation Hazard Zone D) & Worst cases among all TIH, corrosive, Hazard Zone D gases \\
\hline 3355 & Insecticide gas, poisonous, flammable, n.o.s & Worst cases among all flammable, insecticide gases \\
\hline 3355 & Insecticide gas, poisonous, flammable, n.o.s. (Inhalation Hazard Zone A) & Worst cases among all flammable, insecticide, Hazard Zone A gases \\
\hline 3355 & Insecticide gas, poisonous, flammable, n.o.s. (Inhalation Hazard Zone B) & Worst cases among all flammable, insecticide, Hazard Zone B gases \\
\hline 3355 & Insecticide gas, poisonous, flammable, n.o.s. (Inhalation Hazard Zone C) & Worst cases among all flammable, insecticide, Hazard Zone C gases \\
\hline 3355 & Insecticide gas, poisonous, flammable, n.o.s. (Inhalation Hazard Zone D) & Worst cases among all flammable, insecticide, Hazard Zone D gases \\
\hline
\end{tabular}


TABLE 2.7 Summary of Mixtures and Solutions on DOT TIH List and How They Were Treated for the ERG2004

$\mathrm{UN}$

\begin{tabular}{|c|c|c|c|}
\hline Number & Category & Proper Shipping Name & Modeled as \\
\hline \multicolumn{4}{|c|}{ Mixtures and Solutions Modeled as Single Toxic Species } \\
\hline 1040 & Mixture & Ethylene oxide with nitrogen & $100 \%$ ethylene oxide \\
\hline 1051 & Solution & Hydrocyanic acid, aqueous solutions, with more than $20 \%$ hydrogen cyanide & $100 \%$ hydrogen cyanide \\
\hline 1911 & Mixture & Diborane & $7 \%$ diborane \\
\hline 1955 & Mixture & Methyl bromide and nonflammable, nonliquified compressed gas mixtures & $100 \%$ methyl bromide \\
\hline 1612 & Mixture & Hexaethyltetraphosphate and compressed gas mixtures & $20 \%$ hexaethyltetraphosphate \\
\hline 1613 & Solution & Hydrocyanic acid, aqueous solution, with not more than $20 \%$ hydrogen cyanide & $20 \%$ hydrogen cyanide solution in water \\
\hline 1703 & Mixture & Tetraethyl dithiopyrophosphate and gases, in solution & $20 \%$ tetraethyl dithiopyrophosphate \\
\hline 1705 & Mixture & Tetraethyl pyrophosphate and compressed gas mixtures & $20 \%$ tetraethyl pyrophosphate \\
\hline 1744 & Solution & Bromine solutions & $100 \%$ bromine \\
\hline 1754 & Mixture & Chlorosulfonic acid and sulfur trioxide mixture & $100 \%$ sulfur trioxide \\
\hline 1967 & Mixture & Parathion and compressed gas mixtures & $20 \%$ parathion \\
\hline 1975 & Mixture & Nitric oxide and dinitrogen tetroxide mixtures & $100 \%$ nitric oxide \\
\hline 2600 & Mixture & Carbon monoxide and hydrogen mixtures & $100 \%$ carbon monoxide \\
\hline 3294 & Solution & Hydrogen cyanide, solution in alcohol, with not more than $45 \%$ hydrogen cyanide & $45 \%$ hydrogen cyanide solution in alcohol \\
\hline 3300 & Mixture & Carbon dioxide and ethylene oxide mixture, with more than $87 \%$ ethylene oxide & $100 \%$ ethylene oxide \\
\hline 3318 & Solution & Ammonia solution, with more than $50 \%$ ammonia & $100 \%$ ammonia \\
\hline \multicolumn{4}{|c|}{ Mixtures Modeled with Multiple Toxic Chemical Species } \\
\hline 1581 & Mixture & Chloropicrin and methyl bromide mixtures & (i) $5 \%$ chloropicrin, and (ii) $95 \%$ methyl bromide \\
\hline 1582 & Mixture & Chloropicrin and methyl chloride mixtures & (i) $2 \%$ chloropicrin, and (ii) $98 \%$ methyl chloride \\
\hline 1023 & Mixture & Coal gas & $\begin{array}{l}48 \% \text { hydrogen, (ii) } 27 \% \text { methane, (iii) } 10 \% \text { carbon monoxide, } \\
\text { (iv) } 5 \% \text { carbon dioxide, and (v) } 6 \% \text { nitrogen, }\end{array}$ \\
\hline 1071 & Mixture & Oil gas & $\begin{array}{l}\text { (i) } 55 \% \text { hydrogen, (ii) } 25 \% \text { methane, (iii) } 11 \% \text { carbon monoxide, } \\
\text { (iv) } 3 \% \text { carbon dioxide, (v) } 2 \% \text { ethylene, and (vi) } 2 \% \text { benzene }\end{array}$ \\
\hline
\end{tabular}


each entry may have several corresponding entries in the Table. For categories that had fewer than three chemicals from which to pick the worst-case example, the selection pool was enlarged to include materials from the next less restrictive designation. For instance, the pool for the generic category described as compressed gas, poisonous, oxidizing, corrosive, n.o.s. (Inhalation Hazard Zones C and D) included all corrosive gases in Hazard Zones C and D, respectively, because no corrosive, oxidizing gases with those hazard zone designations were identified in the analysis pool.

Table 2.7 lists the mixtures and solutions treated in the ERG2004 analysis and indicates how they were modeled. In general, the distances for mixtures were determined by (1) selecting a surrogate compound, (2) considering the toxic effects of a single hazardous constituent, or (3) considering the toxic effects of multiple hazardous constituents. Surrogates were chosen when the mixture composition was not specified. For these cases, the surrogate was chosen as the worst-case chemical or potential composition in that mixture. As an example, consider chlorosulfonic acid and sulfur trioxide mixtures (UN 1754). In these mixtures, sulfur trioxide is the more hazardous component, primarily because of its higher vapor pressure. The addition of chlorosulfonic acid will act to lower the vapor pressure, so a $100 \%$ sulfur trioxide mixture is the worst case and was chosen for analysis. A single hazardous constituent was modeled for several cases involving mixtures in compressed gases and solutions (e.g., diborane, tetraethyl pyrophosphate, hydrocyanic acid solutions). For each case, the worst case as specified in 49 CFR or by the description was modeled. The third class of mixtures involves compounds with more than one hazardous component. For instance, in chloropicrin and methyl bromide mixtures (UN 1581), chloropicrin is dissolved in methyl bromide up to $5 \%$ by volume. Therefore, toxic effects of both constituents are taken into account. The result is a mixture that behaves almost identically to pure methyl bromide in terms of release rate and dispersion. However, the mixture is much more toxic than pure methyl bromide as a result of the high toxicity of chloropicrin, so the PAD is longer than that for pure methyl bromide.

\subsection{ANALYSIS OF WATER-REACTIVE MATERIALS}

\subsubsection{Background}

Trucks and rail cars that transport hazardous chemicals can have accidents in which their solid or liquid cargo spills into a water-filled roadside ditch, stream, river, lake, or estuary. This presents the possibility that a material that otherwise does not pose an inhalation hazard might react with the water to produce toxic gaseous byproducts. In the following discussions, materials that generate substantial quantities of toxic gases fairly rapidly after a spill into water are referred to as toxic inhalation hazards by water-reaction, or TIHWR ${ }^{2}$. An example of a TIHWR material is silicon tetrachloride, which is not a TIH material but produces airborne hydrogen chloride $(\mathrm{HCl})$ and $\mathrm{HCl}$ mist upon exposure to water. Even heavy rainfall at the time of an accident or airborne water vapor can cause the emission of $\mathrm{TIH}$ gases from this material. The well-known

2 Note that we explicitly exclude from our definition and discussions that follow materials that evolve flammable gases that do not otherwise pose a toxic hazard. 
Chicago spill of silicon tetrachloride from a storage tank in 1976 is an example. At one point in the eight-day episode, heavy rainfall led to a significant increase in emissions and sudden damage to the surroundings because additional $\mathrm{HCl}$ was released into the atmosphere. The same problem, though of less magnitude (due to a smaller release amount), would occur if a truck or tank car spilled silicon tetrachloride into water or had an accident during a rainstorm.

Until recently, little attention was directed to materials that emit gases into the atmosphere when accidentally released into water. Kapias and Griffiths (1999) presented a limited discussion of water-reactive chemicals and the modeling of accidental releases. They used the example of silicon tetrachloride, which is a strong $\mathrm{HCl}$ emitter, as a result of either reacting with liquid water or scavenging atmospheric water vapor. Over the last decade, however, we have identified numerous such materials and recommended them for inclusion in the ERG as part of a long-term program for identifying TIHWR materials and quantifying their emissions.

This section provides a description of our program for identifying and classifying TIHWR materials, as well as a companion experimental program instituted to provide a quantitative basis for the TIHWR analysis. The technical aspects of how the TIH emission rates from these materials were modeled and how they were treated in the statistical analysis as part of the ERG2004 study are detailed in Section 3.3.2.3, and additional detail on the experiments and the parameters employed in the TIHWR modeling appear in Appendixes C and D.

\subsubsection{Identification and Evaluation of Candidates}

To compile the list of water-reactive materials for the Guidebook from the large pool of potential $\mathrm{TIH}$ emitters, we first screened materials by applying general principles of chemical reactivity, seeking specific reactivity information from the chemical literature and comparing such information among related compounds. Screening started with the 208 chemically distinct materials previously found by Carhart et al. (1996) to be capable of producing TIH gases if spilled into a river, lake, or other body of surface water. The 37 materials that make up the 1996 North American ERG "List of Dangerous Water-Reactive Materials" are a subset of these 208. In the analysis for the ERG2000, 16 more materials were added to this list based on review of other water-reactive lists and recommendations by DOT.

The final initial list of 224 candidate materials was carefully evaluated to determine which ones provided sufficient hazards to warrant their inclusion in the Table. The evaluation process consisted of the following steps:

1. Consideration of general patterns of reactivity.

2. Examination of the primary literature.

3. Examination of standard secondary sources such as Kroschwitz (1991-1996) and Lewis (1966). 
4. Experimental tests on compounds to estimate the yield and rate of production of toxic gases when mixed with water. These experiments both confirmed water-reactivity and provided a quantitative basis for TIHWR hazard estimates.

Steps 1 through 3 were used to generate the 1996 TIHWR list. For the ERG2000, the experimental program outlined in Step 4 was initiated. This program was expanded for the ERG2004 to include over half of the TIHWR list. The need for such experimental data is underscored by the fact that quantitative observations of TIH gas evolution from hazardous chemicals added to water do not exist in the chemical literature. The experimental program is outlined below.

\subsubsection{Experimental Program}

The experimental program consisted of a series of small-scale experiments with candidate materials. The materials were tested for the generation of gases by mixing small amounts (about 1 millimole [mmol]) with water in a closed system. The release of gas was measured over time by observing the displacement of a suitable liquid in a manometer. Experiments were conducted using two different amounts of water (the stoichiometric equivalent amount and fivefold molar excess) for each material considered. The experimental procedure and resulting data and analysis are fully described in Appendixes C and D.

Experiments were conducted for 21 candidate materials for the ERG2000 or about onequarter of the total TIHWR list. Heavy emphasis was placed on members of the large class of $\mathrm{HCl}$ emitters. An additional 35 materials were considered for the ERG2004 analysis, bringing the proportion of materials on the TIHWR list for which we have experimental data to over $50 \%$.

The experimental program not only gave useful quantitative information on the rates of evolution of TIH gases, but it also allowed us to delete several chemicals from the TIHWR list because no evolution of gas was observed. In a few such cases, a reaction did actually occur, but the gas that was produced was rapidly dissolved when water was in excess. This effect might not occur when larger quantities are involved, and additional experiments will be required to establish whether significant amounts of TIH gases would escape under such conditions.

\subsubsection{Additional Selection Criteria}

There are a few additional issues associated with the evaluation process that deserve special attention:

- Some potential TIHWR materials, especially generic materials, are not sufficiently described chemically to allow a complete assessment of their behavior when spilled into water. Such materials are not included on the TIHWR list unless there is a positive indication that a TIH gas might develop in a spill into water. Examples of materials that are not included on the 
TIHWR list at this time include: pyrophoric organometallic compound n.o.s. (UN 3203); organometallic compound solution water-reactive, flammable, n.o.s (UN 3207); water-reactive substances, liquid, corrosive, n.o.s. (UN 3129); water-reactive solid, corrosive, n.o.s. (UN 3131); water-reactive substances, liquid, n.o.s, (UN 3148); water-reactive liquid, toxic, n.o.s. (UN 3130); and substances which in contact with water emit flammable gases, solid, poisonous, n.o.s. (UN 3134).

- On the other hand, generic alkyl halides and chlorosilanes might generate sufficient gaseous $\mathrm{HCl}$ or another hydrogen halide to qualify as TIHWR materials. For this reason, aluminum alkyl halides (UN 3052); metal alkyl halides, n.o.s. (UN 3049); chlorosilanes, n.o.s. (UN 2985); chlorosilanes, flammable, corrosive, n.o.s. (UN 2986); and chlorosilanes, corrosive, n.o.s. (UN 2987), are included.

- Materials that are gaseous at ordinary temperatures (with boiling points below $0^{\circ} \mathrm{C}$ at atmospheric pressure) were uniformly not recommended for inclusion. An example is trifluoroacetyl chloride, which boils at $-18^{\circ} \mathrm{C}$. Such compounds would probably boil away too rapidly to allow a significant reaction with surface water under most atmospheric conditions.

- Liquids boiling in the range of ordinary environmental temperature $\left(0-20^{\circ} \mathrm{C}\right)$ presented problems, because they occur as a liquid or a gas depending on water temperature. Cyanogen chloride was rejected because it is a gas at temperatures above most of this range (boiling point $13.1^{\circ} \mathrm{C}$ ) and because it does not require a spill into water to pose a TIH hazard. Chlorine trifluoride (boiling point $11.8^{\circ} \mathrm{C}$ ) was rejected on similar grounds. On the other hand, $\mathrm{BCl}_{3}$ is included despite being a gas at $20^{\circ} \mathrm{C}$, because the reaction of the spilled chemical with water rapidly generates $\mathrm{HCl}$ gas, which is more toxic than the parent compound. Also, since its boiling point is $12.5^{\circ} \mathrm{C}$, the material can remain in the liquid state when spilled into cold water. In addition, $\mathrm{BCl}_{3}$ is frequently purveyed in solution in an organic solvent.

- Materials that undergo highly exothermic reactions with water sometimes generate acidic mists depending on the way that they are mixed with water. This happens when water at hot spots of reactivity boil violently. The resulting seething and bumping kicks colloidal particles of hydrated or partially hydrated material into the air. For example, oleum (UN 1831) quite often raises a fume containing $\mathrm{SO}_{3} \cdot \mathrm{H}_{2} \mathrm{O}, \mathrm{H}_{2} \mathrm{SO}_{4}$, and related acidic species when it is mixed with water. Such a mist presents an obvious inhalation hazard. Similar mists can form when sulfur trioxide (UN 1829) and sulfuryl chloride (UN 1834) mix with water. Materials this category were treated individually. Oleum and $\mathrm{SO}_{3}$ were not included as TIHWR materials because spills into water would likely not lead to protective action distances in excess of those already listed for land-based spills. Sulfuryl chloride was listed as a TIHWR material based on its co-generation of gaseous HCl. Phosphorus 
pentaoxide (UN 1807) might raise an acidic mist (of oxo-acids of phosphorus) in a spill into water. Although mists present a clear toxic inhalation hazard in certain release scenarios, the generation of mists alone is not currently a sufficient criterion for inclusion in the TIHWR table.

- Certain materials dissolve smoothly in water and without generation of gases under most circumstances but can decompose to evolve TIH gases if the mixing with water occurs under conditions that prevent the loss of the heat of dissolution and allow a sufficient rise in temperature. Such conditions were not attained in the water-reactivity experiments (Appendix C).

- Thermal decomposition of calcium hypochlorite (UN 1748) and lithium hypochlorite (UN 1471) generates chlorine and/or $\mathrm{HCl}$ (Lewis 1966, p. 707), which are TIH gases. These two hypochlorites appeared as TIHWR materials in the ERG1996 but were removed as TIHWR materials in the ERG2000 and ERG2004 because the auto-heating scenario appeared too unlikely.

- A similar decomposition of sodium hydrosulfite (UN 1384), calcium hydrosulfite (UN 1923), and zinc hydrosulfite (UN 1931) can occur with water, generating sulfur dioxide and possibly hydrogen sulfide, which are TIH gases. These three hydrosulfites appear as TIHWR in ERG2004 on the basis of the behavior of sodium hydrosulfite in a serious plant accident in New Jersey April 21, 1995 (EPA and OHSA 1997).

\subsubsection{Summary}

As a result of the steps and considerations detailed above, 37 new materials were proposed for inclusion in the ERG2000 and 14 new materials were added to the TIHWR list for 2004, while 5 materials were removed for 2004. The full list of TIHWR materials considered in the ERG2004 is presented in Appendix D, along with a brief summary of reasons for the inclusion of each material. Appendix D also provides the parameters necessary to model TIH evolution such as (1) shipment state, (2) TIH gas(es) evolved, (3) density of the material, (4) stoichiometric yield, (5) overall efficiency factor, and (6) rate constants.

\subsection{DETERMINATION OF INITIAL ISOLATION DISTANCES}

The Initial Isolation Distance is the length of the radius of a circular Initial Isolation Zone around the accident site from which people are to be kept away. Establishment of an Initial Isolation Zone serves two purposes. First, it provides a protective barrier upwind to protect against exposures due to wind direction variations. Second, it defines a zone downwind where life-threatening effects might be expected. The latter is generally a more stringent requirement, so that purpose is used to define the Initial Isolation Distance. 
The Initial Isolation Distance is calculated in an identical manner to the PAD except that lethality is the health end point. As such, distances are evaluated using the 1-h LC 50 . The 1-h value is used in all cases (no time adjustment is made) even though exposure times will be generally be much shorter, since plume meander is a very transient phenomenon and people would not remain in this zone unless they became incapacitated. Earlier efforts using a 5-min exposure time together with the ERPG-3 (or surrogate) yielded slightly longer distances, although the methods are comparable for most materials. We use $\mathrm{LC}_{50}$ values because they are experimentally derived and available for nearly all materials on the TIH list.

It is important to note that many $\mathrm{TIH}$ materials are also flammable or oxidizers. The Initial Isolation Distance does not account for the explosive or flammable nature of the material, so there may be large differences between the Initial Isolation Distance and corresponding isolation distance guidelines in the orange guide pages in the ERG2004. In some cases, the Initial Isolation Distance can be less than the suggested evacuation distance based on flammability or explosion concerns. 


\section{STATISTICAL ACCIDENT SCENARIO ANALYSIS AND CASRAM}

CASRAM (Chemical Accident Statistical Risk Assessment Model) lies at the core of the analysis for PAD determination for the ERG2004 and is the key tool we employ for both constructing accident scenarios and executing the consequence models. CASRAM predicts hazard-zone distributions (i.e., areas in which a threshold chemical concentration is exceeded) and/or affected populations resulting from releases associated with hazardous materials transportation or storage. The model utilizes a variety of statistical data for hazardous materials releases and an extensive meteorological database to statistically generate and analyze release scenarios. For a given health effect (injury, fatality, etc.), hazard-zone distributions are generated stochastically through Monte Carlo sampling of accident scenario parameters (time, location, release amount, meteorology, etc.) and detailed consequence modeling of the hypothetical releases. CASRAM is specifically designed for the statistical analysis of hazardous material release problems. It is this feature, in particular, that separates CASRAM from many other hazardous material release models, such as ALOHA (Reynolds 1992) and HPAC/SCIPUFF (Sykes et al. 1998). Rather than specifying a deterministic measure of risk, CASRAM determines the distribution of possible outcomes, thus allowing identification of the probability of a particular consequence within the limits of the statistical data.

CASRAM is primarily a routing-based risk assessment model that requires shipment attributes (e.g., materials, containers) and shipment routes as inputs. It provides distributions of affected persons as outputs (e.g., Brown et al. 2001). However, it is equally applicable to a geographically based incident distribution system. A geographically based system is required for the ERG analysis because the Table must reflect releases that occur anywhere in North America. The statistical accident scenario analysis (see Figure 2.1) combines the shipment profile information discussed in Section 2.2, meteorological observations from a preprocessed meteorological database, and statistical information from the HMIS database to provide a large distribution of incidents. These incidents are then modeled using the consequence models within CASRAM. The overall goal of this analysis is to identify the distributions of safe distance (i.e., hazard zone length) associated with the transport within North America of all materials that are given in the Table. The Protective Action and Initial Isolation Distances in the Table are the $90^{\text {th }}$-percentile values of these safe-distance distributions.

This section first discusses the statistical scenario analysis and then the meteorological database used in the ERG2004 study. The emission rate and dispersion modeling within CASRAM, which makes up the consequence modeling effort to determine PADs, is then outlined. 


\subsection{STATISTICAL SCENARIO ANALYSIS}

\subsubsection{Overview of Analysis Steps}

For each material in the Table, we use CASRAM to model between 200,000 and $1,000,000$ separate incidents distributed between highway and rail transportation, relevant container types (e.g., DOT class 105 tank car, MC 330 cargo tank, $1 \mathrm{~A} 1$ drums, etc), and release types (i.e., accident-related and en route/nonaccident). The distribution of the incidents within the categories above are specified in the shipment profiles discussed in Section 2.2. Incidents are also distributed geographically and temporally on the basis of transportation mode and release type. Geographic and temporal effects have a large influence on meteorology, which in turn directly affects the safe distance calculation. The location of the incident affects the general climate and land use (e.g., dry desert, temperate farmland), whereas the time of day and month affect the weather at that locale.

Each release modeled in the analysis is assigned a random date, time, and location. The locations for U.S.-based accidents are chosen probabilistically on the basis of state distributions of accidents in the HMIS database. Separate distributions are used for accident-related and en route/nonaccident releases for both highway and rail. Locations for Canada and Mexico are based on population density. Date and time are assigned on the basis of month-of-year and timeof-day distributions for incidents in the HMIS database as outlined in Section 3.1.4, where the year is assigned in a five-year window that corresponds to observations in the meteorological database $\left(1985-1989^{3}\right)$.

The emission rate model in CASRAM uses the shipment information and meteorology as specified above to determine the rate at which specific materials are released into the atmosphere. The first step is to estimate the discharge fraction on the basis of historical statistical distributions generated from analysis of incidents in the HMIS database. The emission rates of the material to the atmosphere are then calculated using physical models for discharge, flashing, and evaporation applicable to that release. Within the emission rate model, the total amount of material spilled (discharge fraction) and pool size are treated stochastically.

Using the emission rates for the chemical(s) involved and the ambient meteorology, the dispersion model within CASRAM then determines the affected areas. We view the dispersion calculation as a two-step process. Step 1 involves characterizing the meteorology from the available surface observations, and step 2 involves estimating the transport and dispersion from the applicable meteorological parameters. Step 1 is accomplished with a meteorological preprocessor, which is a series of algorithms that take raw meteorological data (e.g., wind speed, temperature, humidity, cloud cover) and site information (e.g., land cover type, roughness length) and calculate parameters necessary for estimating dispersion. While this analysis is usually closely associated with dispersion modeling, the atmospheric parameters calculated are

3 More current meteorological data are not employed since the 1985-1989 window is the last 5-year period in which manual observations are available. Current NWS observations are made with automated equipment which has difficulty discerning cloud amounts and precipitation type. 
also used in estimating source emission rates. Often, these parameters are represented as stability classes; however, in this analysis, the turbulence and structure of the atmospheric boundary layer were represented by more fundamental turbulence measures, such as friction velocity, surface heat flux, and inversion height. Step 2 is accomplished in CASRAM with a Lagrangian integral dispersion model for passive releases, coupled with a dense gas dispersion model to address large releases of liquefied gases where heavier-than-air plumes form. The physical relationships that make up the emission rate and dispersion models are outlined in Sections 3.3 and 3.4, respectively.

\subsubsection{The HMIS database}

The Hazardous Materials Information System (HMIS) database, maintained by DOT, catalogues transportation-related incidents involving the release of hazardous materials. In latter years, about 15,000 incidents per year are catalogued for highway, rail, air, and waterway transportation. In general, $80-85 \%$ of the incidents are highway-related, and about $11-14 \%$ are rail-related. Air accidents (mainly luggage-related) comprise almost all of the rest. Incidents in the database can occur (1) during an accident (i.e., during a vehicular mishap or a train derailment), (2) en route but not during an accident (e.g., due to a cargo shift or valve failure), or (3) during loading or unloading operations. For highway transportation, about $80 \%$ of the incidents in the HMIS database occur during loading and unloading; for rail, about $85 \%$ are en route/nonaccident releases.

For each incident catalogued in the HMIS database, information pertaining directly to the hazardous material release is provided, including: (1) name of chemical shipped, (2) container type and capacity, (3) number of containers shipped, (4) number of containers that fail, and (5) amount of material released. Multiple chemicals released during the same incident are recorded in the database as separate records. The database also contains information concerning the occurrence of fire, explosion, water immersion, and environmental damage as well as the number of deaths, major and minor injuries, and persons evacuated. Death and injury statistics pertain only to the consequence of the hazardous material release and not to physical trauma due to the accident itself. Since this database is comprised of data from actual hazardous materials incidents, it is an invaluable tool in statistical analyses of hazardous material transportation incidents and the best publicly available source of information concerning container failure statistics and release amount. For the ERG2004 analysis, as well as other risk assessments, we use HMIS data to specify geographical incident distributions, temporal incident distributions, and discharge fraction distributions as described in the subsections below. Further information on the HMIS database and our previous analyses can be found in (Brown et al. 2001).

\subsubsection{Geographic Incident Distributions}

In the ERG2004 analysis, accidents are distributed across all 50 states in the United States, the southern Canadian provinces, and Mexico. Within the United States, the accident distribution is based on incident distributions in the HMIS database for 1985-2002, and separate geographic distributions are used for highway and rail. The distribution of accidents 
between U.S., Canada, and Mexico is based on the relative Gross Domestic Products (GDP) for 2002. GDP was selected as a measure for hazard materials incidents because of the unavailability of detailed hazardous materials incident data for Mexico. For the United States and Canada, the relative GDP for chemical and chemical products is similar to the total GDP, but because the industry-specific GDP was not available for Mexico, and because Canadian and U.S. chemicalspecific GDPs are calculated slightly differently, the total GDP was considered to be the more robust indicator. On the basis of this breakdown, 89\% of incidents modeled in the ERG2004 analysis occurred in the United States, 7\% occurred in Canada, and 4\% occurred in Mexico. Within Canada and Mexico, incidents were distributed into regions on the basis of population. Canada was divided by province. In this process, New Brunswick, Newfoundland, Nova Scotia, and Prince Edward Island were combined as the "Atlantic Provinces," and the Yukon and Northwest Territories were excluded because of their small populations (less than $0.5 \%$ of total Canadian population). Mexico was divided into three regions: Northern (above $22^{\circ} \mathrm{N}$ latitude), Central (between 18 and $22^{\circ} \mathrm{N}$ latitude), and Southern (below $18^{\circ} \mathrm{N}$ latitude, including the Yucatan Peninsula). A breakdown of the geographic distribution of incidents in the ERG2004 analysis for highway and rail transportation is provided in Table 3.1.

\subsubsection{Temporal Incident Distributions}

Temporal release distributions are important to specify appropriate meteorology. In some risk assessment studies, temporal variables also influence the population at risk since (1) population density of a particular location can change throughout the day and (2) a greater fraction of people are outside during the daytime. Two temporal variables are defined in our analysis: hour of day and month of year. Concerning meteorology, hour of day is critical due to the diurnal cycle of the atmospheric boundary layer and month of year is important due to climatological effects on temperature, wind speed, and daytime mixing height. Note that the wind speed and temperature affect not only dispersion but also the evaporation rate of spilled liquids to the atmosphere.

Temporal incident statistics in CASRAM and used in the ERG2004 analysis are based on HMIS database records for 1990-2002 and are updated from the distributions used for the ERG2000. These are shown in Figures 3.1 and 3.2. The figures show the percent of incidents broken into time of day as shown in Figure 3.1 and month of year Figure 3.2. Data are shown for

accident-related and en route/nonaccident releases for both highway and rail. The number of incidents in the statistical samples is provided in the figure legends. The incident sample is slightly different between the figures, due to missing data fields (i.e., hour or month) in a small percentage of HMIS incident records.

\subsubsection{Discharge Fraction Distributions}

In the CASRAM emission model, the discharge fraction is estimated using statistical distributions developed from an analysis of the HMIS database. HMIS records list container type, number of containers shipped, number of containers that fail, and the amount of material 
TABLE 3.1 Geographic Distribution of Highway and Rail Transportation Incidents Modeled in the ERG2004 Analysis, by Percentage

\begin{tabular}{llllll}
\hline \multicolumn{1}{c}{ State } & Rail & Highway & \multicolumn{1}{c}{ State/Province } & Rail & Highway \\
\hline Alabama & & & & & \\
Alaska & 2.49 & 2.13 & New Mexico & 1.06 & 1.26 \\
Arizona & 0.18 & 0.10 & New York & 0.86 & 2.13 \\
Arkansas & 1.34 & 1.37 & North Carolina & 2.23 & 2.71 \\
California & 2.57 & 2.87 & North Dakota & 0.17 & 0.18 \\
Colorado & 10.63 & 4.25 & Ohio & 4.37 & 5.53 \\
Connecticut & 1.54 & 1.49 & Oklahoma & 0.76 & 1.63 \\
District of Columbia & 0.06 & 0.79 & Oregon & 1.49 & 0.93 \\
Delaware & 0.00 & 0.03 & Pennsylvania & 1.54 & 5.10 \\
Florida & 0.12 & 0.20 & Rhode Island & 0.02 & 0.15 \\
Georgia & 1.54 & 2.36 & South Carolina & 0.71 & 1.58 \\
Hawaii & 2.42 & 2.65 & South Dakota & 0.02 & 0.20 \\
Idaho & 0.00 & 0.05 & Tennessee & 2.35 & 3.29 \\
Illinois & 0.73 & 0.39 & Texas & 12.18 & 7.89 \\
Indiana & 5.06 & 4.98 & Utah & 2.40 & 1.24 \\
Iowa & 1.57 & 2.70 & Vermont & 0.01 & 0.14 \\
Kansas & 0.77 & 1.15 & Virginia & 1.26 & 1.83 \\
Kentucky & 1.96 & 1.87 & Washington & 1.44 & 1.22 \\
Louisiana & 1.68 & 2.20 & West Virginia & 0.61 & 0.90 \\
Massachusetts & 6.05 & 3.22 & Wisconsin & 0.29 & 1.50 \\
Maryland & 0.23 & 1.20 & Wyoming & 2.59 & 0.54 \\
Maine & 0.63 & 1.40 & Canada and Mexico & & \\
\cline { 3 - 3 } & 0.12 & 0.27 & British Columbia, Can. & 0.82 & 0.82 \\
Minnesota & 3.14 & 1.91 & Alberta & 0.63 & 0.63 \\
Mississippi & 0.50 & 1.45 & Saskatchewan & 0.25 & 0.25 \\
Missouri & 0.86 & 1.60 & Manitoba & 0.27 & 0.27 \\
Montana & 2.37 & 3.04 & Ontario & 2.51 & 2.51 \\
Nebraska & 0.54 & 0.30 & Quebec & 1.71 & 1.71 \\
Nevada & 1.65 & 0.57 & Atlantic Provinces & 0.58 & 0.58 \\
New Hampshire & 0.97 & 0.40 & Northern Mexico & 1.03 & 1.03 \\
New Jersey & 0.01 & 0.23 & Central Mexico & 2.69 & 2.69 \\
\hline & 0.68 & 1.67 & Southern Mexico & 0.76 & 0.76 \\
\hline
\end{tabular}

released, allowing us to statistically characterize the release amount for containers of various types and sizes. In this process, we found that the fraction of the container capacity that is released proved a robust statistic in terms of both collapsing data from a wide variety of containers and ease of use in subsequent risk assessment studies. This fraction of the total container capacity that is released is defined here as the discharge fraction. Since the amount of material actually shipped is not currently provided in the HMIS database, the container capacity provides the best estimate of the amount shipped. As an example, consider a vehicular accident that involves the shipment of chlorine in a 17,000-gal Type $105 \mathrm{~A} 300 \mathrm{~W}$ rail car and in which $240 \mathrm{gal}$ is released. The discharge fraction would be $240 / 17,000$ or 0.014 . As is discussed later, this incident would be grouped with all other incidents involving releases from pressurized rail cars (Types 105 and 112) to construct the discharge fraction distributions for accident-related releases. 


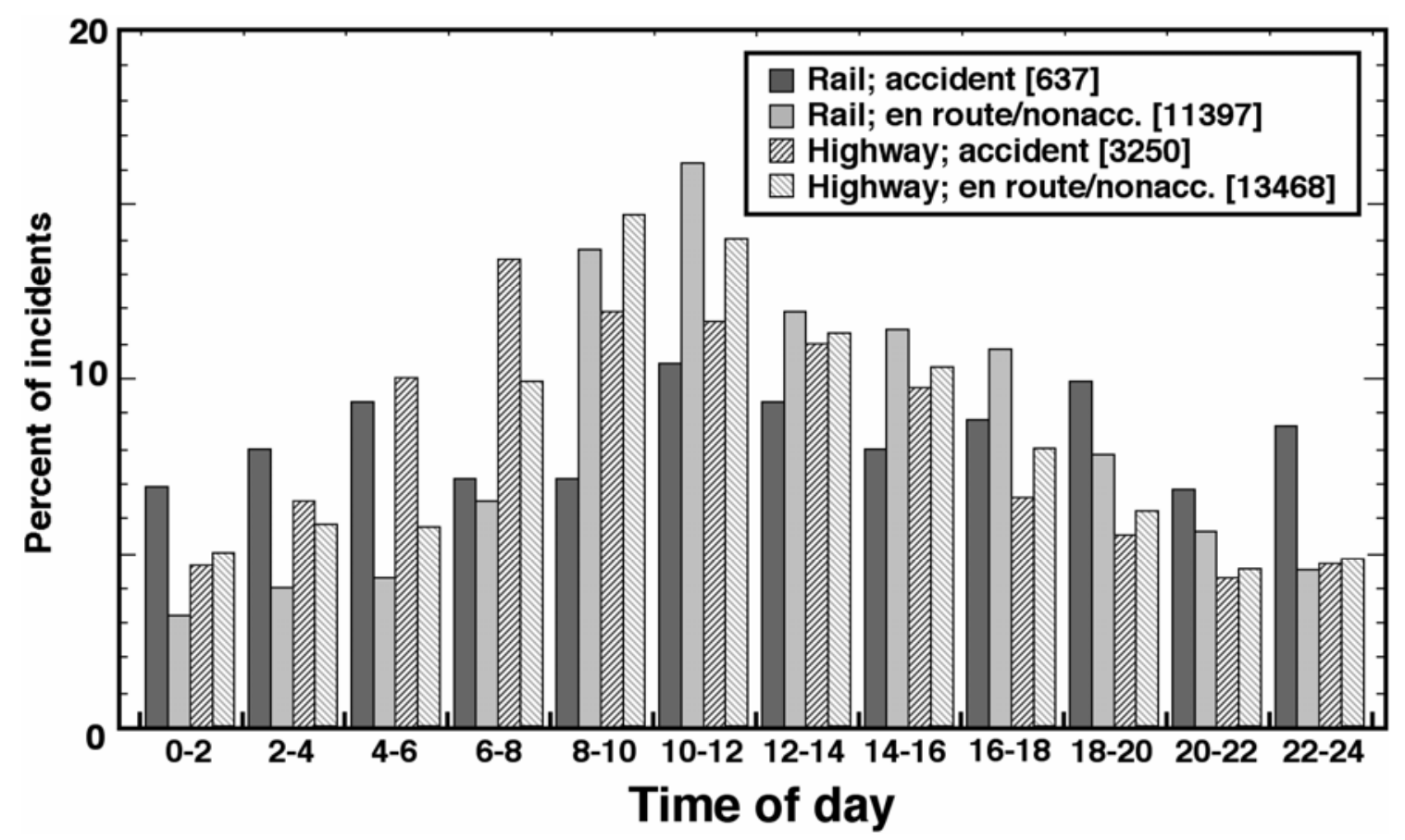

FIGURE 3.1 Hourly Distributions of Rail and Highway Transportation-Related Hazardous Material Releases Occurring During Accident-Related and En Route/Nonaccident Incidents from the HMIS Database, 1990-2002

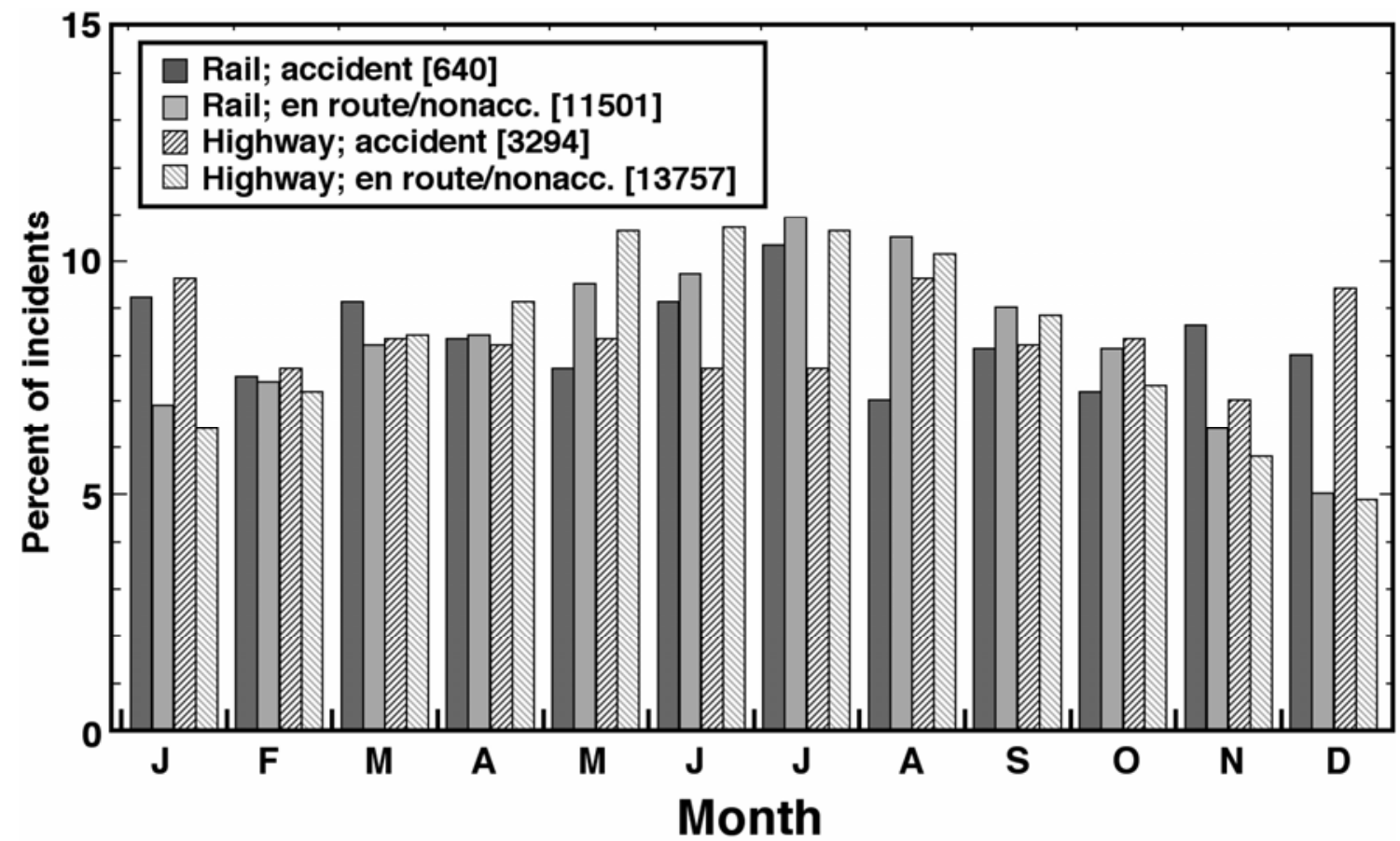

FIGURE 3.2 Monthly Distributions of Rail and Highway Transportation-Related Hazardous Material Releases Occurring During Accident-Related and En Route/Nonaccident Incidents from the HMIS Database, 1990-2002 
In the CASRAM source model, discharge fraction statistics are segregated according to container type. In general, these container types can be classified according to whether they are used for bulk or package freight. As part of the analysis for the ERG2004, we re-evaluated our previously published discharge-fraction distributions (Brown et al. 2001) using HMIS data through 2002. Our basic methodology for organizing bulk container discharge fractions is unchanged our from ERG2000 analysis, although the distributions are slightly different due to the longer window of HMIS data employed, and the distributions are now represented with continuous equations. Substantial changes have been made in the organization of package freight statistics, however, which have had a moderate impact on PAD estimates for some materials, especially those authorized only for package freight transportation. We shall discuss bulk and package freight separately below.

\subsubsection{Bulk Containers}

Discharge fraction statistics for bulk containers are shown in Figures 3.3 and 3.4. Figure 3.3 shows discharge fraction distributions for containers that are used for nonpressurized and lowpressure materials. Figure 3.4 shows the distributions for containers that are used for highpressure materials. Note that not all container types shown are authorized for transport of TIH materials; these are provided for comparison and for use in risk assessments involving other types of hazardous materials. Discharge fraction distributions are provided for the two transportation-related phases considered in the ERG2004 analysis: accident-related and en route/nonaccident releases. Discharge fraction statistics for bulk package freight were developed for a specific container type (Type 111A tank cars, MC/DOT 312 tanks, etc.) when sufficient statistical data were available (i.e., data on at least 25 incidents). In practice, discharge fractions for other container types for which data are insufficient could be estimated using a surrogate for a similar container type for which data do exist. For example, the surrogate discharge fraction distribution for Type 103 tank cars would be that for Type 111A tank cars, and the surrogate for MC/DOT 338 cargo tanks would be MC/DOT 331 cargo tanks.

To facilitate their use in computational models, we developed mathematical expressions for discharge fraction that can be employed in Monte Carlo analyses. We believe use of these functional forms are preferable to using the raw discrete distributions as had been done previously since they eliminate discretization problems that sometimes occur in the raw distributions. Distributions for bulk containers were fit to the following functional form:

$$
F(\gamma)=\min \left(1, e^{\zeta(\gamma)}\right)
$$

where

$$
\zeta(\gamma)=\sum_{n=1}^{N} a_{i} \gamma^{n b}
$$



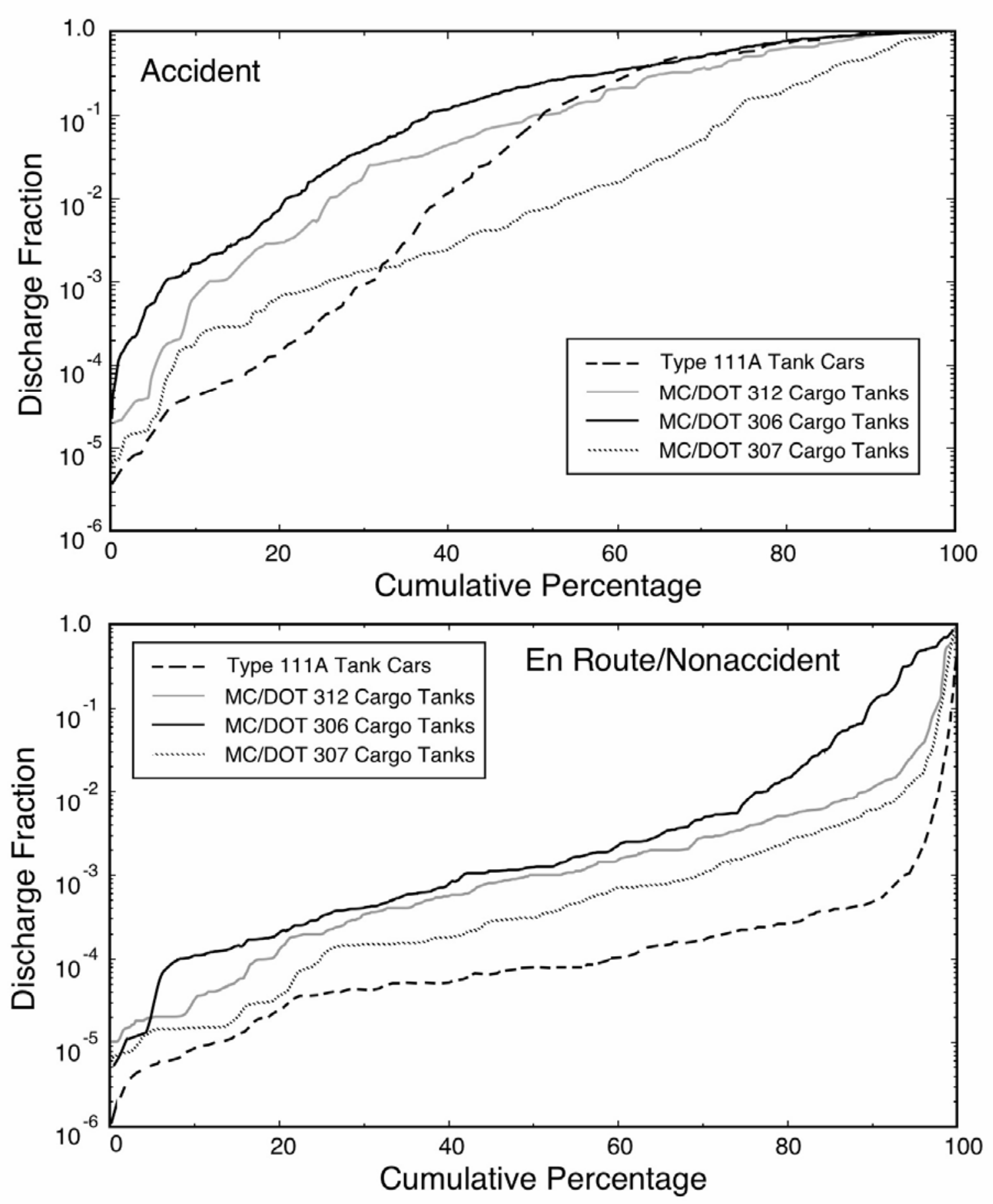

FIGURE 3.3 Discharge Fraction Cumulative Probability for Bulk Nonpressurized and Low-Pressure Containers as Derived from the Analysis of the HMIS Database (1990-2002) for the ERG2004 Analysis. Results are shown for accident-related releases (upper) and en route/nonaccident releases (lower). 

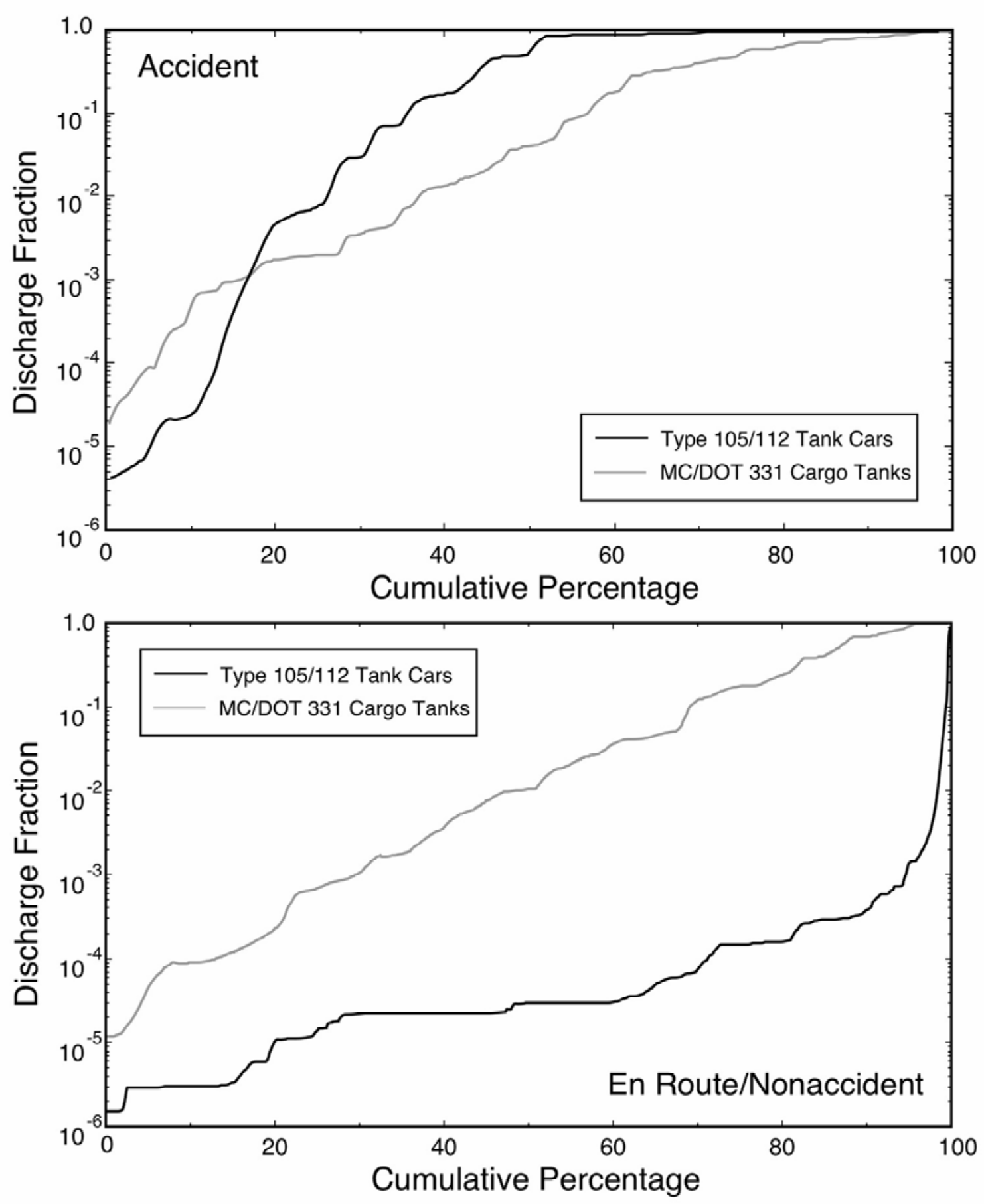

FIGURE 3.4 Discharge Fraction Cumulative Probability for Bulk Pressurized Containers as Derived from the Analysis of the HMIS Database (1990-2002) for the ERG2004 Analysis. Results are shown for accident-related releases (upper) and en route/nonaccident releases (lower). 
Here, $\mathrm{F}$ is the discharge fraction, $\gamma$ is a uniform random deviate from 0 to 1 , and $a_{i}$ and $b$ are coefficients that depend on container type and incident type. $N$, the number of coefficients $a_{i}$ $(i=1, N)$ necessary is either 6 or 8 , depending on the complexities of the distribution, particularly near the ends. The coefficient $b$ serves to stretch the very low probability end of the distribution and is necessary to accurately capture the frequency of rare en route/nonaccident events where a large fraction of the container capacity is released while correctly representing the remainder of the distribution. For all accident-related events, $b=1$, and for en route/nonaccident events, $b<0.5$. The coefficients for Eq. 3.1 for accident and en route/nonaccident events are listed in Tables 3.2 and 3.3, respectively.

\subsubsection{Package Freight Containers}

For package freight containers, we previously developed discharge fraction distributions for accident-related releases that specified the total amount released as a fraction of the total container capacity (i.e., capacity of all containers combined). Discharge fraction distributions for en route/nonaccident releases were represented as the fraction of the capacity of an individual container in the shipment that is released (see Brown et al. 2001). One drawback of this release fraction normalization was that the distributions did not properly represent shipments containing only a few containers. For the ERG2004 analysis, we revised this framework for drums and cylinders based on our re-analysis of HMIS data through 2002 to specify both (1) the percentage of containers that leaked or failed and (2) the discharge fraction distribution applicable to each failed container. In addition, for accident-related incidents, we specified a probability that the entire shipment was released. Since almost all cases of practical interest in evaluating inhalation hazards from package freight shipments involve drums or cylinders, we limited our discharge fraction framework to these two classes of containers.

We divide our discussion below into accident-related and en route/nonaccident releases since the methods employed to treat these cases are substantially different.

\section{Accident-Related Releases}

The first step in the analysis framework is to determine the percentage of cases in which the entire shipment amount is released. Typically, only a few containers of a multiple-container shipment are compromised in a traffic accident. However, in more serious accident-related events, the entire shipment can be released. A complicating factor, though, is that the probability of the entire shipment being released decreases as the number of containers in the shipment increases. Table 3.4 shows the number of accident-related incidents cataloged in HMIS from 1990-2002 involving metal drums and shows the number of these cases in which over $90 \%$ of the total shipment amount was released. Over half of the incidents involving a single metal drum involved the release of the entire shipment contents, whereas for shipments containing multiple drums, the percentage of such incidents decreases to $10 \%$. Table 3.5 shows the identical information for cylinders indicating a similar trend. Note that the total incident counts are rather low, especially for cylinders, so there is significant statistical noise in these data. 
TABLE 3.2 Coefficients for Calculating Discharge Fraction Cumulative Probability Distributions for Accident-related Releases for a Variety of Containers. Coefficients were Developed from the analysis of the HMIS Database (1990-2002) for the ERG2004 Analysis.

\begin{tabular}{|c|c|c|c|c|c|c|c|c|c|}
\hline Container & $\mathrm{b}$ & $\mathrm{a}_{1}$ & $a_{2}$ & $a_{3}$ & $\mathrm{a}_{4}$ & $a_{5}$ & $a_{6}$ & $a_{7}$ & $\mathrm{a}_{8}$ \\
\hline $\begin{array}{l}\text { Type 111A } \\
\text { Tank Cars }\end{array}$ & 1 & -12.2825 & 25.2135 & -97.7815 & 382.86 & -468.648 & -106.407 & 513.26 & -235.777 \\
\hline $\begin{array}{l}\text { Type } 105 / 112 \\
\text { Tank Cars }\end{array}$ & 1 & -13.5011 & 29.0148 & 64.2486 & -235.971 & 223.212 & -65.2201 & 0 & 0 \\
\hline $\begin{array}{l}\text { MC/DOT } 306 \\
\text { Cargo Tanks }\end{array}$ & 1 & -8.02475 & 6.20446 & 104.348 & -325.183 & 284.727 & 149.822 & -358.121 & 146.299 \\
\hline $\begin{array}{l}\text { MC/DOT } 307 \\
\text { Cargo Tanks }\end{array}$ & 1 & -12.5422 & 50.5658 & -169.235 & 257.71 & -84.3257 & -130.654 & 108.216 & -19.6658 \\
\hline $\begin{array}{l}\text { MC/DOT } 312 \\
\text { Cargo Tanks }\end{array}$ & 1 & -11.3375 & 43.4848 & -101.806 & 149.477 & -112.577 & 32.859 & 0 & 0 \\
\hline $\begin{array}{l}\text { MC/DOT } 331 \\
\text { Cargo Tanks }\end{array}$ & 1 & -10.8601 & 43.6839 & -181.754 & 440.336 & -466.95 & 175.992 & 0 & 0 \\
\hline
\end{tabular}

TABLE 3.3 Coefficients for Calculating Discharge Fraction Cumulative Probability Distributions for En Route/Nonaccident Releases for a Variety of Containers. Coefficients were Developed from the analysis of the HMIS Database (1990-2002) for the ERG2004 Analysis.

\begin{tabular}{|c|c|c|c|c|c|c|c|c|c|}
\hline Container & $\mathrm{b}$ & $a_{1}$ & $a_{2}$ & $a_{3}$ & $\mathrm{a}_{4}$ & $a_{5}$ & $a_{6}$ & $a_{7}$ & $a_{8}$ \\
\hline $\begin{array}{l}\text { Type 111A } \\
\text { Tank Cars }\end{array}$ & 0.5 & 0.624664 & -44.0097 & 39.2814 & 150.665 & -334.474 & 50.9987 & 318.078 & -194.708 \\
\hline $\begin{array}{l}\text { Type } 105 / 112 \\
\text { Tank Cars }\end{array}$ & 0.33 & 20.2316 & -171.317 & 379.325 & -285.744 & -97.0904 & 46.2262 & 278.699 & -184.156 \\
\hline $\begin{array}{l}\text { MC/DOT } 306 \\
\text { Cargo Tanks }\end{array}$ & 0.33 & 4.67339 & -71.6677 & 378.743 & -859.395 & 610.244 & 368.827 & -653.445 & 210.363 \\
\hline $\begin{array}{l}\text { MC/DOT } 307 \\
\text { Cargo Tanks }\end{array}$ & 0.36 & 4.46934 & -41.2228 & 51.6474 & -6.432 & -32.2012 & 5.55689 & 11.7468 & -5.51775 \\
\hline $\begin{array}{l}\text { MC/DOT } 312 \\
\text { Cargo Tanks }\end{array}$ & 0.4 & 4.15943 & -39.2797 & 54.9507 & -21.9882 & -17.3163 & 5.09395 & 16.6091 & -13.8431 \\
\hline $\begin{array}{l}\text { MC/DOT } 331 \\
\text { Cargo Tanks }\end{array}$ & 0.5 & 0.338613 & 0.285349 & -6.47588 & -5.23065 & 0 & 0 & 0 & 0 \\
\hline
\end{tabular}


TABLE 3.4 Incident Counts Involving

Drums in Accident-related Releases in the HMIS Database (1990-2002) Shown with the Number of Which Involved Over 90\% of the Total Shipment Amount

Being Released

\begin{tabular}{ccc}
\hline $\begin{array}{c}\text { Number of } \\
\text { Drums in } \\
\text { Shipment }\end{array}$ & $\begin{array}{c}\text { Total Number } \\
\text { of Incidents }\end{array}$ & $\begin{array}{c}\text { Number with } \\
>90 \% \text { Release }\end{array}$ \\
\hline & & \\
1 & 25 & 14 \\
$2-5$ & 26 & 3 \\
$6-10$ & 36 & 3 \\
$10-20$ & 42 & 3 \\
$20-50$ & 27 & 1 \\
$>50$ & 37 & 4 \\
\hline
\end{tabular}

TABLE 3.5 Incident Counts Involving Cylinders in Accident-related Releases in the HMIS Database (1985-2002) Shown with the Number of Which Involved Over $\mathbf{9 0 \%}$ of the Total Shipment Amount Being Released

\begin{tabular}{ccc}
\hline $\begin{array}{c}\text { Number of } \\
\text { Drums in } \\
\text { Shipment }\end{array}$ & $\begin{array}{c}\text { Total Number } \\
\text { of Incidents }\end{array}$ & $\begin{array}{c}\text { Number with } \\
>90 \% \text { Release }\end{array}$ \\
\hline & & \\
1 & 22 & 12 \\
$2-5$ & 14 & 2 \\
$>5$ & 13 & 3 \\
\hline
\end{tabular}

To account for these maximum release events in a robust manner, we first select a fraction of the incidents for which the entire shipment is released. The percentages of such incidents are $10 \%$ and $25 \%$ for shipments of drums and cylinders, respectively. In practice, these are the limiting cases as the number of containers becomes large. The increase in the percentage of total releases where over $90 \%$ of the shipment is released that occurs for shipments involving small numbers of containers is accounted for in the second analysis step detailed below.

For the remainder of the shipments, we then specify the number of failed containers $N_{f}$ as

$$
N_{f}=N_{s} \gamma^{N_{s}^{a}}
$$


where $N_{S}$ is the number of containers shipped, $\gamma$ is a uniform random deviate from 0 to 1 , and a is a coefficient. In this expression, $N_{f}$ is an integer that is rounded up from the value on the right hand side.

The total amount of material released is then written as

$$
F=\min \left(1, \sum_{i=1}^{N_{f}}\left[c_{1}+c_{2} \gamma_{i}+c_{3} \gamma_{i}^{d_{3}}\right]\right),
$$

where $\gamma_{\mathrm{i}}$ is a set of independent (i.e., uncorrelated) uniform random deviates from $i=1, \mathrm{~N}_{\mathrm{f}}$, and $c_{1}, c_{2}, c_{3}$, and $d_{3}$ are constants, the values of which along with $a$ in Eq. 3.2 above are provided in Table 3.6. Note that each failed container is treated independently in Eq. 3.3.

\section{En Route/Nonaccident Releases}

From a statistical perspective, en route/nonaccident events are fundamentally different from accident-related events. Like bulk transportation, the total release amounts are typically very small compared with accident-related releases. En route/nonaccident events are also much more common, as evidenced from HMIS statistics presented previously in Table 2.1. Usually, only one container is involved, even in shipments with large numbers of containers. Tables 3.7 and 3.8 provide incident counts involving drums and cylinders, respectively, from en route/nonaccident releases in the HMIS Database (1990-2002) together with the number that involved only one container and, for comparison with Tables 3.4 and 3.5, the number in which over $90 \%$ of the total shipment amount was released.

To treat the discharge fraction estimation problem for these events, we take a similar approach as we did for accident-related events. We first split out those events where only one container is involved. For both drums and cylinders, we set this as $75 \%$ of incidents. For the other $25 \%$ of incidents, we calculate the number of failed containers with Eq. 3.2 with a different values for the coefficient $a$. Then for all incidents, we use Eq. 3.3 again with different values for the coefficients. The coefficients for Eqs. 3.2 and 3.3 for en route/nonaccident releases are provided in Table 3.9.

\subsection{METEOROLOGICAL DATABASE USED TO PREPARE THE GUIDEBOOK}

The meteorological database is a critical component of the ERG2004 analysis since it provides the historical meteorological data necessary to model hazardous material incidents anywhere in the United States, Canada, and Mexico. This database was generated directly from five years of observational data from 105 cities in the United States and with supplemental data from several stations in Canada and Mexico. The data were first preprocessed with the Surface Energy Budget Meteorological (SEBMET) model (Brown 1997; Brown and Dunn 1998). This meteorological preprocessor was designed for use with statistical dispersion studies such as the 
TABLE 3.6 Coefficients for Calculating Discharge Fraction Cumulative Probability Distributions in Eq. 3.3 for Accident-related Releases Involving Drums and Cylinders. Coefficients were developed from the analysis of the HMIS Database (1990-2002) for the ERG2004 analysis.

\begin{tabular}{lccccc}
\hline & & & & & \\
Container Type & $\mathrm{a}$ & $\mathrm{c}_{1}$ & \multicolumn{1}{c}{$\mathrm{c}_{2}$} & $\mathrm{c}_{3}$ & $\mathrm{~d}_{3}$ \\
\hline & & & & & \\
Drums & 0.5 & 0 & 0 & 2.0 & 1.5 \\
Cylinders & 0.65 & 0 & 0.167 & 3.1 & 3.5 \\
\hline
\end{tabular}

TABLE 3.7 Incident Counts Involving Drums In En Route/Nonaccident Releases in the HMIS Database (1990-2002) Shown with the Number that Involved Only One Container and the Number with Over $90 \%$ of the Total Shipment Amount Being Released (for comparison with Table 3.4)

\begin{tabular}{cccc}
\hline $\begin{array}{c}\text { Number of } \\
\text { Drums in } \\
\text { Shipment }\end{array}$ & $\begin{array}{c}\text { Total } \\
\text { Number of } \\
\text { Incidents }\end{array}$ & $\begin{array}{c}\text { Number } \\
\text { Involving One } \\
\text { Container }\end{array}$ & $\begin{array}{c}\text { Number with } \\
>90 \% \\
\text { Release }\end{array}$ \\
\hline & & & \\
1 & 730 & 730 & 63 \\
2 & 335 & 291 & 3 \\
$3-5$ & 552 & 491 & 2 \\
$5-10$ & 464 & 403 & 1 \\
$10-20$ & 399 & 345 & 0 \\
$20-50$ & 437 & 337 & 0 \\
$>50$ & 705 & 472 & 0 \\
\hline
\end{tabular}

TABLE 3.8 Incident Counts Involving Cylinders in En Route/Nonaccident Releases in the HMIS Database (1990-2002) Shown with the Number that Involved Only One Container and the Number with Over $90 \%$ of the Total Shipment Amount Being Released (for comparison with Table 3.4)

\begin{tabular}{cccc}
\hline $\begin{array}{c}\text { Number of } \\
\text { Cylinders in } \\
\text { Shipment }\end{array}$ & $\begin{array}{c}\text { Total } \\
\text { Number of } \\
\text { Incidents }\end{array}$ & $\begin{array}{c}\text { Number } \\
\text { Involving } \\
\text { 1 Container }\end{array}$ & $\begin{array}{c}\text { Number with } \\
>90 \% \\
\text { Release }\end{array}$ \\
\hline 1 & 37 & 37 & 16 \\
$2-5$ & 45 & 38 & 0 \\
$>5$ & 43 & 28 & 1 \\
\hline
\end{tabular}


TABLE 3.9 Coefficients for Calculating Discharge Fraction Cumulative Probability Distributions in Eq. 3.3 for En Route/Nonaccident Releases Involving Drums and Cylinders. Coefficients were developed from analysis of the HMIS Database (1990-2002) for the ERG2004 analysis.

\begin{tabular}{lccccc}
\hline Container Type & $a$ & $c_{1}$ & $c_{2}$ & $c_{3}$ & $d_{3}$ \\
\hline & & & & & \\
Drums & 0.75 & 0.0025 & 0 & 1.23 & 4.0 \\
Cylinders & 0.75 & 0 & 0.167 & 3.5 & 4.5 \\
\hline
\end{tabular}

ERG2004 analysis. A meteorological preprocessor contains a series of algorithms that use routinely measured observations of wind speed, temperature, humidity, and cloud cover, along with upper-air temperature soundings, to calculate the key atmospheric-boundary-layer parameters necessary for modeling source thermodynamics, transport, and dispersion. The following sections first discuss the raw meteorological data used in the analysis and then provide a brief overview of SEBMET's methodology. We then describe a companion database employed to estimate water temperature for analysis of TIHWR materials.

\subsubsection{Meteorological and Site Data}

For the ERG2004 analysis, CASRAM uses a meteorological database that includes hourly meteorological parameters from 105 U.S. cities from 1985 through 1989 (as illustrated in Figure 3.5) and supplemental data from several stations in Canada and Mexico. The stations were chosen to provide a roughly uniform coverage area. Raw meteorological data included yearly surface files listing hourly values of wind speed, temperature, cloud cover (height and fraction), dew point temperature, pressure, and visibility, among other variables. In addition, upper air data from 80 stations across North America were employed to evaluate the atmospheric temperature profile up to the 7,000-m altitude necessary for determining the daytime mixing height. Site characteristics determined for each station included land cover, vegetative types, the monthly leaf area index (a measure of the canopy density) for each vegetative type, roughness length, albedo, and soil types. These site-specific parameters were determined through a climatological study of the areas surrounding each site.

\subsubsection{Meteorological Preprocessor}

The meteorological preprocessor contains two primary components: a surface energy budget (SEB) model that determines the surface-layer turbulence parameters and an integral model that determines inversion height in convective conditions. Each component is briefly outlined below. Brown (1997) and Brown and Dunn (1998) contain additional information on the meteorological preprocessor, including details on its development and validation. 


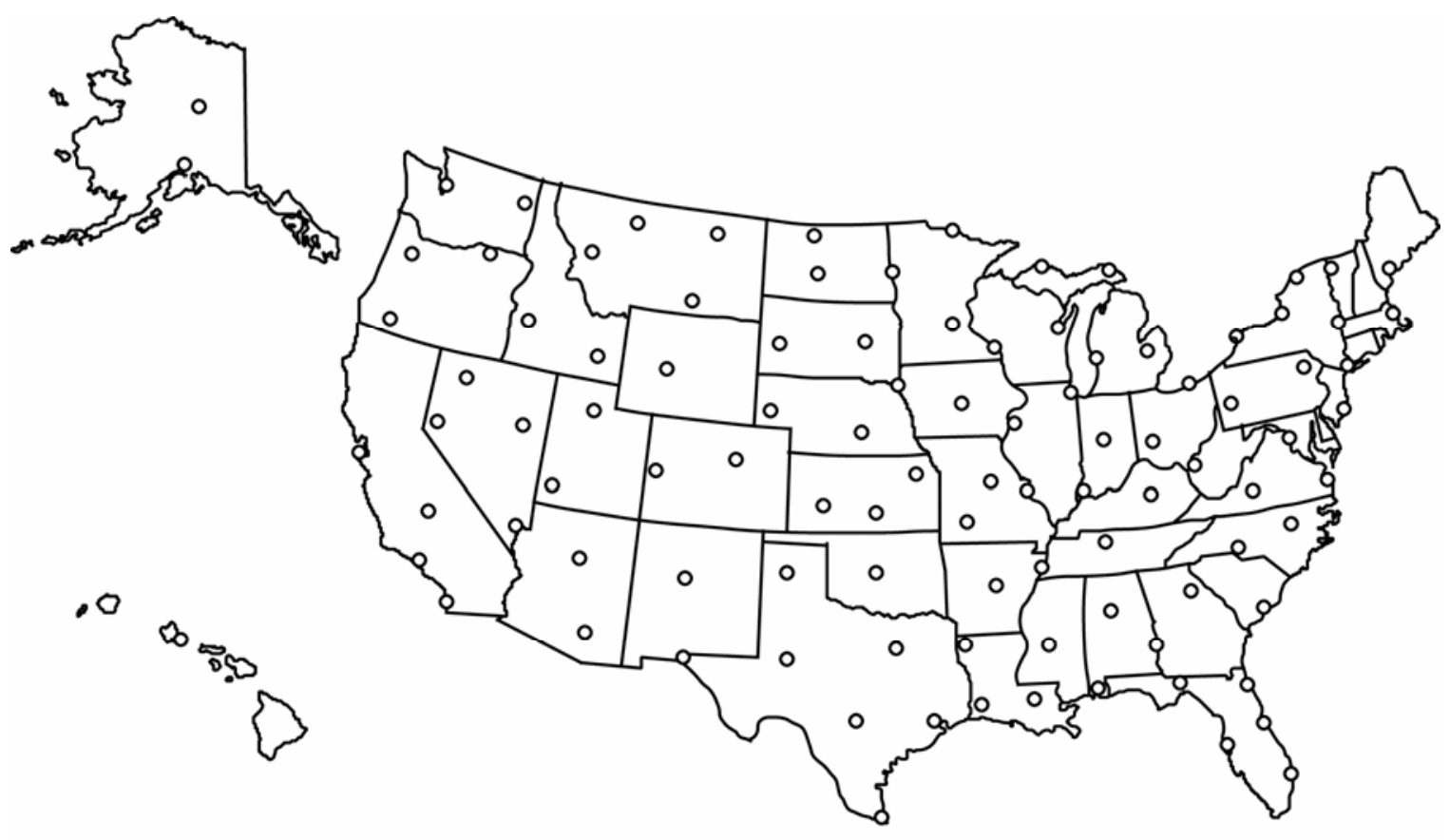

DBA 10017

FIGURE 3.5 Locations of the 105 National Weather Service Stations Used for Statistical Meteorological Characterization

\subsubsection{Surface Turbulence Parameters}

The surface-layer parameters are determined using a SEB model that consists of parameterizations of the various SEB components and well-known flux-profile relationships. The goal of this modeling approach is to isolate the sensible heat flux $H$ from the other energy budget components. The starting point for this analysis is the SEB at the ground. When advection, photosynthesis, and snow melt are neglected, the SEB is most simply represented as follows:

$$
Q^{*}=H+\lambda E_{w}+G+Q_{a},
$$

where

$$
\begin{aligned}
& Q^{*}=\text { net surface radiative heat flux, } \\
& G=\text { conductive soil heat flux, } \\
& \lambda=\text { heat of vaporization for water, }
\end{aligned}
$$


$E=$ evaporation rate (together, $\lambda E_{\mathrm{w}}$ is the latent heat flux), and

$Q_{a}=$ anthropogenic heat flux.

The net surface radiative heat flux is the residual from the absorbed solar radiation $S$, incoming long-wave radiation $L^{+}$, and outgoing long-wave radiation $\varepsilon \sigma T_{\mathrm{s}}^{4}$. It is written as follows:

$$
Q^{*}=\left(1-\alpha_{s}\right) S+L^{+}+\varepsilon_{s} \sigma T_{s}^{4},
$$

where

$$
\begin{aligned}
& \alpha_{s}=\text { surface albedo, } \\
& \varepsilon_{s}=\text { surface emissivity }, \\
& \sigma=\text { Boltzmann constant, and } \\
& T_{s}=\text { surface temperature. }
\end{aligned}
$$

At the surface, the short-wave balance is always positive, while the outgoing long-wave radiation generally exceeds incoming long-wave radiation. The model is constructed by parameterizing each of the components in the SEB in terms of routinely observed meteorological observations and site characteristics. A brief description of the SEB component parameterizations is presented below.

Incoming solar radiation at the surface is determined using a variation of the Parameterization C model (Iqbal 1983). In this model, the solar radiation incident on top of the atmosphere is determined from earth-sun relationships and then adjusted via transmittance functions to account for atmospheric absorption and scattering. Separate transmittances are specified for (1) absorption due to (a) ozone, (b) water vapor, and (c) aerosols and for (2) scattering due to (a) Rayleigh particles (molecules) and (b) aerosols. In addition, the model accounts for multiple reflections of solar radiation between the ground and the atmosphere, which significantly increase solar radiation when the surface albedo is high (e.g., cloud cover coupled with deserts or snow cover). Along with data on location and time, the model requires data on (1) local atmospheric pressure; (2) surface albedo; (3) visibility; (4) precipitable water content, which is derived from upper-air data; and (5) ozone amount, which is estimated from latitudinal-seasonal averages. Attenuation due to cloud cover is estimated from the opaque and total cloud cover and cloud height by a semi-empirical model that was developed by Brown (1997) from an analysis of the National Solar Radiation Database (NREL 1992).

Net long-wave radiation at the surface is determined from data on surface temperature, emissivity, near-surface temperature profiles, and humidity profiles. In particular, incoming long-wave radiation is estimated on the basis of a semi-empirical relationship developed from parameterizing long-wave radiation estimates from a detailed narrow-band radiation model. This relationship relies on data on the near-ground temperature profile, $10-\mathrm{m}$ water vapor pressure, 
cloud fraction, and cloud height. Outgoing long-wave radiation is represented directly using ground and foliage temperatures that are derived in the model.

At the surface, the solar radiation and incoming long-wave radiation are balanced against the (1) sensible heat transfer, (2) latent heat transfer, (3) ground conduction heat transfer, and (4) outgoing long-wave radiation. To provide a physical, unified treatment valid under both daytime and nighttime conditions, SEBMET employs a two-layer canopy model that evaluates energy transfer components from both the ground and the vegetative layer. The model is formulated by writing separate energy-balance relationships for the ground and the vegetative canopy similar to Eqs 3.4 and 3.5 and by parameterizing the energy transfer components in terms of available meteorological measurements and known vegetation and ground characteristics. The vegetative layer is characterized by the leaf area index and the bulk stomatal resistance, which is a measure of the latent heat transfer resistance. The key parameter is the bulk stomatal resistance, which embodies the physiological response of the vegetation to the ambient environment. In the SEBMET canopy model, this parameter is estimated by modeling the stomatal responses to (1) ambient temperature, (2) vapor pressure deficit, (3) solar radiation, and (4) moisture stress. Evaporation from the soil is considered by defining a moisture store in the soil layer that is reduced through evaporation and replenished by precipitation. The outgoing long-wave radiation is estimated directly from the canopy and ground temperatures and the canopy coverage percentage. Heat conduction into the ground is estimated using a numerical, finite-difference algorithm that allows the dependence of soil properties and the insulating effect of overlying vegetation and snow to be explicitly treated. Such a scheme allows the ground temperature profile to be saved for use in pool evaporation calculations in the CASRAM emission rate model.

\subsubsection{Inversion Height and Boundary Layer Height}

The inversion height in convective condition is estimated with a one-dimensional model of the atmospheric boundary layer based on the Driedonks slab model (Driedonks 1982). In the Driedonks model, as in similar models, the surface turbulence fluxes $u_{*}$ and $H$ are integrated over time so the boundary layer evolves from an initial early-morning height. The Driedonks model was chosen because of its comprehensive treatment of dynamics at the inversion, favorable comparison with field data, and ease of inclusion in the preprocessor.

In neutral and stable conditions, the boundary layer height is less well defined. Generally, the boundary layer height, at least in stable conditions, is taken to be the height at which surfaceinduced turbulence drops to a negligible value. Here, the diagnostic relations for stable

conditions defined by Nieuwstadt (1981) and provided by the neutral limit $(h=0.3 u * / f$, where $f$ is the Coriolis force) are used, following the recommendations of Hanna and Paine (1989). (Hanna and Paine recommend that the neutral relation be used when $L$ is greater than or equal to 100 and that Nieuwstadt's relation be used when $L$ is more than zero but less than 100.) 


\subsubsection{Water Temperature for TIHWR Reaction Rates}

As discussed in Section 3.3.3, the most sensitive environmental variable affecting reaction rates of spilled chemicals with water is temperature. In general, the reaction rate roughly doubles for every $10^{\circ} \mathrm{C}$ increase in temperature. To account for this effect in our statistical hazardous materials analysis, we developed a database for water temperature as a function of location and Julian day. We investigated the following three sources of water temperature data.

1. Great Lakes Buoys (GLBs). Data were available from eight buoys scattered throughout the Great Lakes, whose reported temperatures yielded a multiyear average that was parameterized as a function of Julian day (Lesht and Brandner 1992).

2. U.S. Geological Survey (USGS) Water Quality Network (WQN). The USGS gathered data on stream water temperatures for periods of up to 30 years before 1990 at about 680 water stations (the WQN data). The data were for uneven durations and taken at uneven frequencies. In the best cases, monthly temperature values were recorded for a period of 20 years or more. For most stations, the day of the month and the time of day on which measurements were recorded varied, and about one month elapsed between measurements.

3. National Oceanic and Atmospheric Administration (NOAA) Ocean Harbor Buoys. Data from a series of harbor buoys along the Atlantic and Pacific Coasts were recorded for years. Data from a representative set of locations were examined and found to substantively agree with the USGS data as a function of latitude. Therefore, the harbor buoy data were not specifically used for this study.

In the ERG2004 analysis, we added 10 more land-based (WQN) stations to bring the total to 45 land-based and $8 \mathrm{GLB}$ stations, the locations of which are shown in Figure 3.6. These stations were selected to meet several criteria. We wanted the network of stations to cover the United States evenly or, if concentrated, to represent population centers. We desired data from a period of $20 \mathrm{yr}$ to permit meaningful climatological modeling. While the target was about 240 temperature values, we chose stations that had recorded between about 150 and 450 values.

We used a special parameterization of average GLB values by Julian day published by Lesht and Brandner (1992) for this study. For each WQN station or harbor buoy, the full set of values was fitted to the following equation:

$$
T(J)=T_{\text {avg }}+(\Delta T) \sin \left(2 \pi \frac{J-J_{o}}{365}\right) .
$$

The values of $T_{a v g}, \Delta T$, and $J_{o}$ were fitted to all of the values. The GLBs were fitted with a sixparameter function given by Lesht and Brandner (1992). Because the formula has six adjustable 


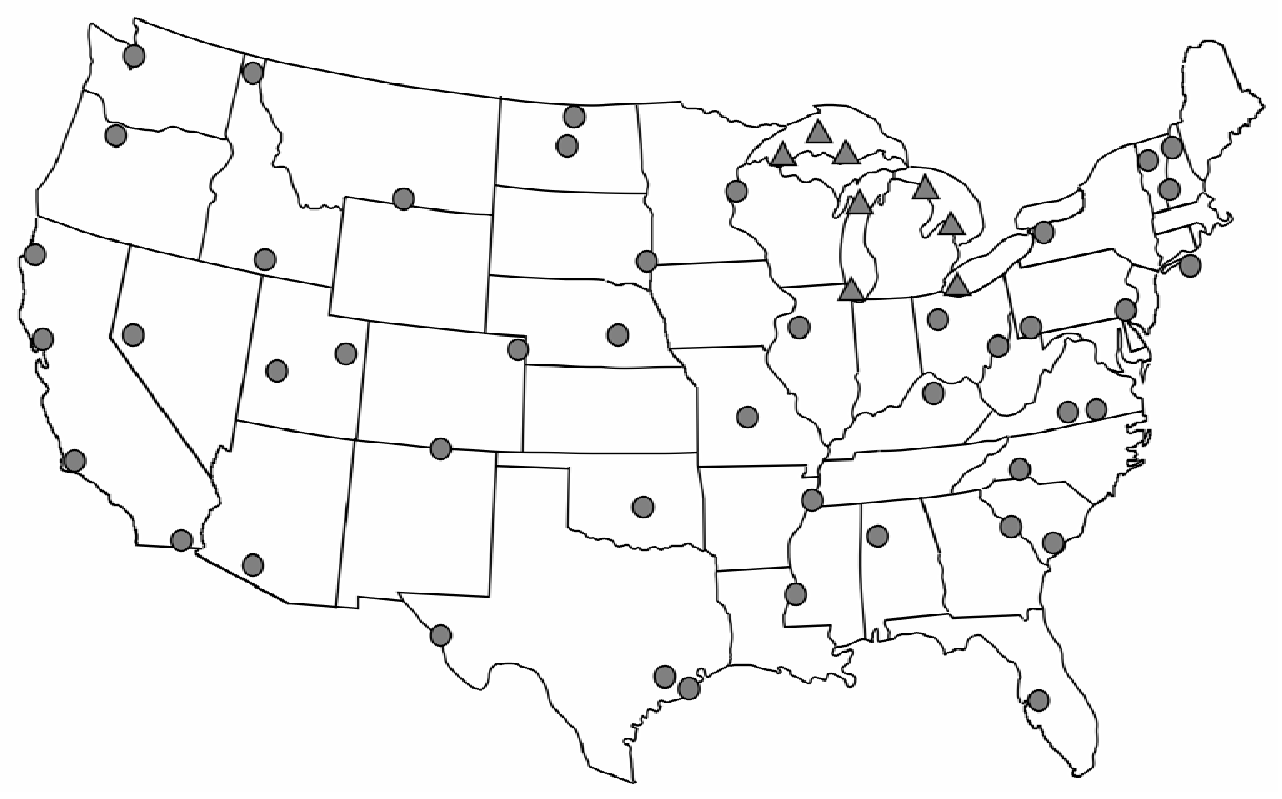

FIGURE 3.6 Water Quality Network Stations Used to Determine Variations of Average Water Temperature by Julian Day and Location (circles = 45 landbased sampling sites; triangles $=8$ GLBs)

constants and is somewhat detailed, it is not provided here. Interested readers are referred to Lesht and Brandner (1992).

\subsection{EMISSION RATE CHARACTERIZATION}

\subsubsection{Overview of Release Types}

Materials are shipped as either (1) solids, (2) ordinary liquids, (3) compressed gases, or (4) liquefied gases. The emission rate of a chemical to the atmosphere is largely dependent on the shipment state. Due to their low volatility, solids typically exhibit low emission rates. Therefore, with the exception of certain water-reactive materials, no solid materials appear on the ERG TIH list. Release mechanisms for the other shipment states (2-4 above), together with water-reactive materials, are illustrated in Figure 3.7 and discussed next.

Liquid materials are emitted to the atmosphere through pool evaporation as illustrated in Figure 3.7, panel a. As shall be discussed shortly, the pool evaporation rate is dependent on many factors, in particular the vapor pressure of the material. For volatile liquids (high vapor pressure) the evaporation rate is often limited by the available energy, whereas for low-volatility liquids, wind speed and atmospheric stability become very important. Highly volatile liquids evaporate very quickly, sometimes cooling the pool in excess of $30^{\circ} \mathrm{C}$ below the ambient temperature. For certain materials, the evaporating pool can cool so much that it freezes. 


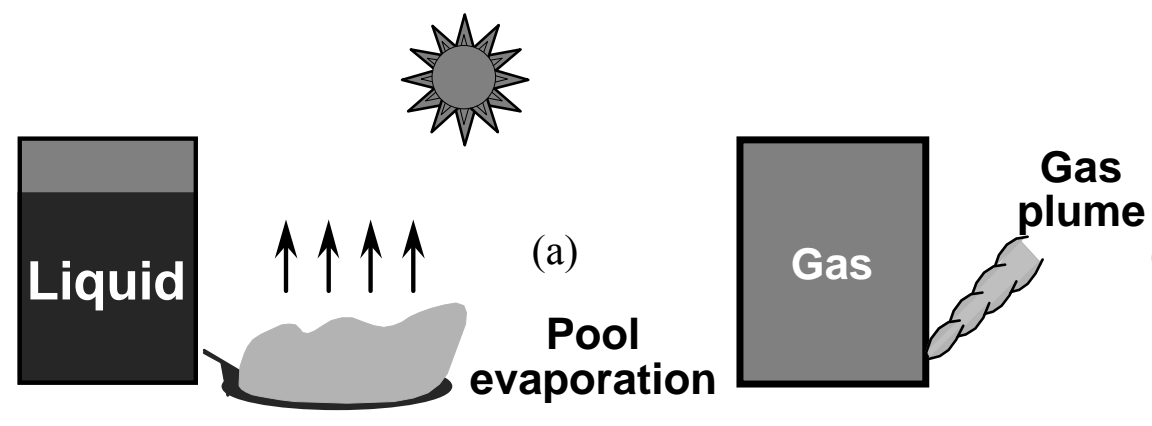

(b)

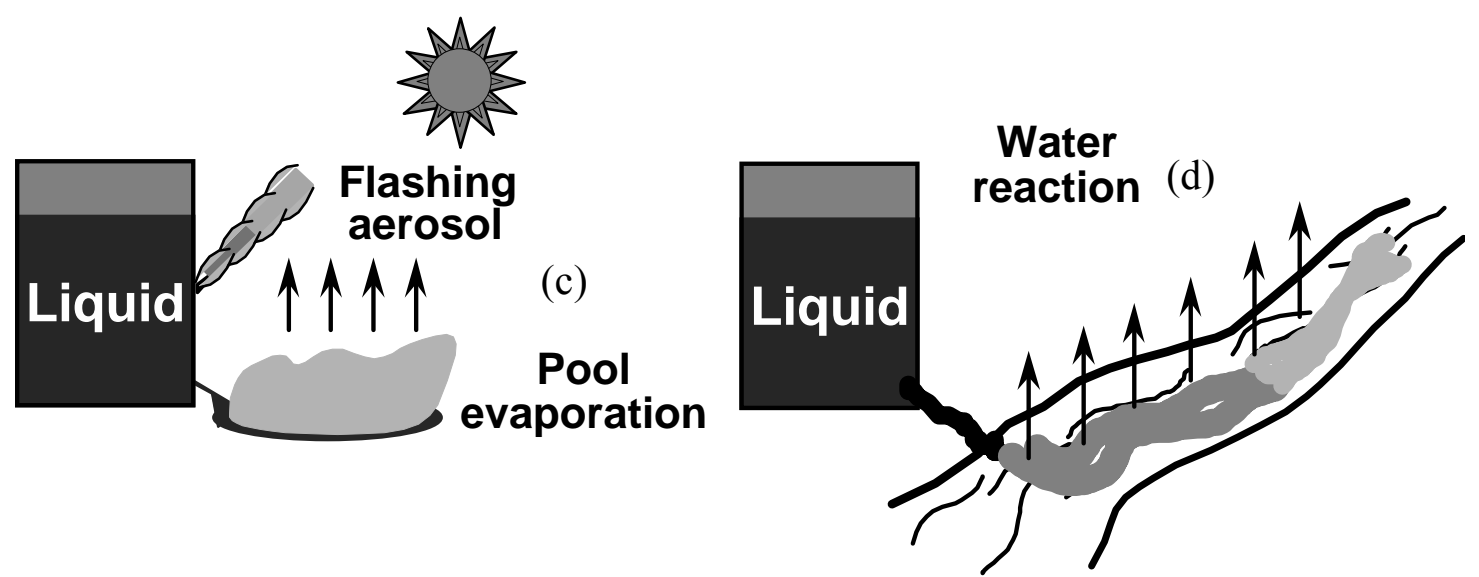

FIGURE 3.7 Important Source Types for Inhalation Risk Considered in the ERG2004 Analysis ( $a$ = ordinary liquids, $b=$ compressed gases, $\mathrm{c}=$ liquefied gases, $\mathrm{d}=$ water-reactive materials)

Compressed gases are released in a so-called "blowdown" process as illustrated in Figure 3.7, panel b. The blowdown process usually empties the container rapidly and, in the case of severe accidents, may result in a near-instantaneous release. All else being equal, release rates for compressed gases are many times higher than for ordinary liquids. Exceptions to this rule include valve leaks and minor cracks, for which release rates may be very small. Valve leaks are the most common release mechanism for en route/nonaccident releases. With the exception of hydrogen, compressed gases are usually shipped in cylinders smaller than 100 gal.

The most catastrophic releases involve liquefied gases as is illustrated in Figure 3.7, panel c. These materials are gases at atmospheric pressure, but are liquefied through pressurization and, in some cases, refrigeration. For these materials, the release is broken into two phases. In the initial phase, denoted as the flashing and entrainment phase, a fraction of the material (up to $30 \%$ of the total) is instantaneously vaporized upon exiting the vessel due to the sudden reduction of pressure. Due to the rapid expansion of the material, much of the remaining liquid exiting the vessel is broken into tiny drops forming an aerosol. The flashed vapor typically entrains substantial quantities of this aerosol, with the larger droplets quickly settling to the ground or "raining out." This aerosol usually evaporates quickly when exposed to air, cooling the vapor/air mixture to the point where the density of the mixture can become considerably heavier than air. The second phase involves evaporation of the liquid that falls to the ground. Since the 
vapor pressures for such materials are usually well above atmospheric pressure, the evaporation phase is usually short in comparison to that for materials that are liquids at atmospheric pressure. Liquefied gas releases yield the largest overall emission rates in comparison to liquid and compressed-gas releases and generally represent the greatest danger to the public.

An additional class of materials discussed throughout this report and treated in the ERG analysis is water-reactive materials (Figure 3.7, panel d). These materials release toxic gases to the atmosphere through the reaction of a parent chemical with water. Therefore, a water-way entry or an additional water exposure mechanism is required for them to become an inhalation hazard. The release rate of the toxic by-product to the atmosphere depends primarily on chemical reactivity, although many other factors can also influence the reaction rate. Key among these is water temperature, for higher water temperatures can greatly accelerate the reaction rate. Evidence also suggests water acidity may be important. Mixing rates also have a clear effect, for greater mixing will generally accelerate the reaction rate. Rivers are generally more turbulent than some other bodies of water and will enhance mixing and thus increase the TIH gas evolution rate. Also, whether the parent chemical is heavier or lighter than water (solids and some liquids) can affect the mixing rate. Finally, highly exothermic water reactions will result in faster reactions through heating and mixing and also release mists (typically acidic), which can be very corrosive and toxic.

\subsubsection{CASRAM Emission Model and Its Application to the ERG2004 Analysis}

The source component of CASRAM determines hazardous material release rates for each of the spill and/or vaporization scenarios described above. Information on the shipment, location, and meteorology are employed in the emission rate model to determine the amount of material spilled and the release rate. First, the discharge fraction is estimated using statistical distributions generated from analysis of HMIS database incidents, as discussed in Section 3.1.2. Then one or more physical models are employed to estimate chemical discharge rates and evaporation rates.

\subsubsection{Assumptions Used to Develop Accident Scenarios}

In developing the accident scenarios, the following assumptions were used:

- For accident-related releases from rail tank cars involving a container breach ( $60 \%$ of total set of such releases), the release rate of material from the container is regulated by the size of the hole, which is specified statistically according to hole size distributions presented by Raj and Turner (1993). Since no comparable data exist for highway transportation, the hole size distributions for highway bulk containers are set to those for tank car types having similar specifications (wall thickness, insulation). Total release amounts are limited to conform with discharge fraction distributions in the HMIS database by varying the location of the hole in the container. 
- For en route/nonaccident incidents and for package freight accident-related incidents, release amounts are determined directly from the HMIS database release fraction distributions. For these incidents, the release is assumed to occur uniformly over a period of $15 \mathrm{~min}$. This method overpredicts the impacts from slow leaks. However, this has no effect on values appearing in the Table, since slow vapor releases typically involve the release of small amounts of material and fall well below the $90^{\text {th }}$-percentile level of protection cutoff.

- Liquids released from the container that are not flashed or entrained with the flashed liquid form a pool on the ground that expands and contracts in response to gravity-driven fluid flow and evaporation. For highway-related releases, $50 \%$ of spills are assumed to occur on paved surfaces. For rail releases, $25 \%$ of spills are assumed to occur on pavement or roadbed. All other spills are assumed to occur on natural surfaces characteristic of the accident locale. Ground temperature profiles to a depth of $1 \mathrm{~m}$ are provided in the preprocessed meteorological database to facilitate the ground conduction calculation, which is very important for high volatility liquids.

- The discharge rate of solid water-reactive materials from their containers is varied so that $18 \%, 40 \%, 60 \%$, and $95 \%$ of the total release amount is discharged in the first 5, 15, 30, and $60 \mathrm{~min}$, respectively. The total release amounts are selected to conform with discharge fraction distributions in the HMIS database. The time-dependent emission rate of the TIH by-product from the water is calculated from the container release rate and the reaction rate of the chemical with water.

- Except for the reaction of water-reactive materials to produce TIH byproducts, chemical transformation is not considered.

- The temperature of the material upon release is taken as a weighted average of the air temperature and $290 \mathrm{~K}$, with the exact weight depending on the container type.

\subsubsection{Physical Considerations}

\section{Discharge from Tanks}

For liquids and liquefied gases, the first step in this process is determining the timedependent discharge rate from the tank. Tanks punctured below the liquid line release their contents according to the Bernoulli equation (Perry et al. 1984), namely,

$$
Q_{l}=c_{o} A_{h} \rho_{l}\left[2 g \Delta h+2\left(\frac{P_{t}-P_{a}}{\rho_{l}}\right)\right]^{\frac{1}{2}},
$$


where

$$
\begin{aligned}
& Q_{l}=\text { liquid release rate }(\mathrm{kg} / \mathrm{s}), \\
& c_{o}=\text { discharge coefficient, } \\
& A_{h}=\text { hole area }\left(\mathrm{m}^{2}\right), \\
& \rho_{l}=\text { liquid density }\left(\mathrm{kg} / \mathrm{m}^{3}\right), \\
& g=\text { gravitational acceleration }\left(9.81 \mathrm{~m} / \mathrm{s}^{2}\right), \\
& \Delta h=\text { height of liquid above the hole }(\mathrm{m}), \\
& P_{t}=\text { tank pressure }(\mathrm{Pa}), \text { and } \\
& P_{a}=\text { atmospheric pressure }(\mathrm{Pa}) .
\end{aligned}
$$

Equation 3.7 is used to calculate the discharge rates from all bulk containers in CASRAM, where $A_{h}$ is provided by the hole size distributions given by Raj and Turner (1993). Concerning the discharge coefficient $c_{O}$, experimental data show that $c_{O}$ depends on the exit velocity, fluid viscosity, and hole characteristics (Perry et al. 1984). However, at high exit velocities, characteristic of the incidents modeled in CASRAM, $c_{O} \approx 0.6$. Therefore, we set $c_{O}$ to 0.6 for all releases modeled in CASRAM. If the material is a liquid, it forms a parabolic (in depth) pool on the ground or pavement, which expands and contracts in response to gravitydriven fluid flow and evaporation. Liquefied gases, on the other hand, equilibrate to atmospheric pressure through flashing (explosive evaporation of a fraction of the released material). A fraction of the remaining material is aerosolized and entrained in the flashed vapor, and the remaining material falls to the ground and evaporates.

\section{Pool Evaporation}

Pool evaporation within CASRAM is determined using a time-dependent, energy-budget model that accounts for the important air-pool-ground energy fluxes that govern evaporation rate. The emission rate of material from an evaporating pool $\mathrm{Q}_{\mathrm{e}}$ is represented as

$$
Q_{e}=A_{p} E_{c}
$$

where

$$
\begin{aligned}
& A_{p}=\text { pool area }\left(\mathrm{m}^{2}\right) \text { and } \\
& E_{c}=\text { evaporation rate from the pool }\left(\mathrm{kg} / \mathrm{s} \cdot \mathrm{m}^{2}\right) .
\end{aligned}
$$


Assuming that the ambient concentration of the evaporating chemical is zero, the evaporation rate is a function of the chemical vapor pressure and transfer coefficients, such that

$$
E_{c}=h_{m} \frac{P_{v}\left(T_{p}\right)}{R T_{p}},
$$

where $h_{m}$ is the mass transfer coefficient, $P_{V}$ is the chemical vapor pressure at the pool temperature $T_{p}$, and $R$ is the ideal gas constant for air. The mass transfer coefficient $h_{m}$ is a function of pool size, meteorology, and chemical properties. The key variable in Eq. 3.9 is the pool temperature, for the vapor pressure on which the evaporation rate depends usually varies exponentially with temperature. Pool temperature, and hence the evaporation rate, is determined using a time-dependent, energy-budget model that considers heat transfer to and from the pool via radiation, convection, conduction, and evaporation. In particular, the conductive heat flux from the ground is especially important and often the dominant source for available energy, especially for very volatile liquids. Treating the conduction correctly, especially the initial and boundary conditions, was a principal motivation in the development of the CASRAM evaporation model.

The energy budget of the pool is a balance between solar radiation $S^{+}$, incoming longwave radiation $L^{+}$, outgoing longwave radiation $\varepsilon_{p} \sigma T_{p}{ }^{4}$, convective heat transfer $H_{p}$, latent heat transfer of water vapor from plant material $\lambda E_{w p}$, conductive heat transfer $G_{p}$, evaporative heat loss $h_{f g} E_{\mathrm{c}}$, and pool energy storage $m c_{p} d T_{p} / d t$ ( $p$ subscripted variables refer to pool-specific quantities except $c_{p}$ ). Considering these processes, we write the pool energy budget as

$$
Q_{p}^{*}=H_{p}+\lambda E_{w p}+G_{p}+h_{f g} E_{c}+m c_{p} \frac{d T_{p}}{d t}
$$

where $Q_{\mathrm{p}}^{*}$ is the net radiation given by

$$
Q_{\mathrm{p}}^{*}=\left(1-\alpha_{\mathrm{p}}\right) S^{+}+L^{+}+\varepsilon_{p} \sigma T_{p}^{4}
$$

and $G_{p}$ is the ground heat flux, to the pool given by Fourier's law as

$$
G_{p}=-k_{g}\left(\frac{d T}{d z}\right)_{z=0} .
$$

In these relationships, $m$ is the pool mass per unit area, $t$ is time, $z$ is depth into the ground, $\varepsilon_{\mathrm{p}}$ is the pool emissivity, $\alpha_{\mathrm{p}}$ is the pool albedo, $k_{g}$ is the ground heat conductivity, and $\sigma$ is the Boltzmann constant $\left(5.67 \times 10^{-8}\right)$. The necessary transfer coefficients for evaporation are provided by a chemical property database and the preprocessed meteorological database. In particular, the meteorological quantities of interest include aerodynamic resistances based on stability and wind speed, air temperature, incoming solar and longwave radiation, and the initial ground temperature profile. The relative importance of the terms in Eq. 3.10 depends largely on the volatility of the chemical in the pool. For highly volatile liquids (i.e., ones that quickly 
evaporate), the evaporative cooling term is large and must be balanced by available energy provided by the net radiation, convective heat transfer, and ground conduction terms. Therefore, the amount of available energy often limits the evaporation rate for these materials. For lowvolatility liquids, the evaporative cooling term is small, and the remaining terms are similar to what they would be in the absence of a pool. In these materials, evaporation is governed by the vapor pressure, wind speed, and surface turbulence characteristics.

\section{Compressed Gas Releases}

In CASRAM, compressed gas releases are modeled using semi-empirical blowdown relationships based on compressible-flow theory. Releases are assumed to be isentropic rather than isenthalpic. The isentropic assumption is that heat transfer to the vessel is negligible, which is a reasonable approximation for most accidental releases. The isenthalpic approximation, on the other hand, requires sufficient heat transfer to maintain isothermal conditions (assuming $c_{p}$ is constant).

For compressed gas releases, the release rate is calculated on the basis of hole size, tank pressure, and gas density such that

$$
Q=c_{o} A_{h}\left[P_{t} \rho_{g} \gamma\left(\frac{2}{\gamma+1}\right)^{\frac{\gamma+1}{\gamma-1}}\right]^{\frac{1}{2}},
$$

where $\gamma$ is the specific heat ratio $c_{p} / c_{V}$. As in the case of liquid releases, $c_{O}$ is set to 0.6 for all releases.

Equation 3.13 is applicable until the tank pressure drops below a critical value, given as

$$
P_{r}=\left(\frac{\gamma+1}{2}\right)^{\frac{\gamma}{\gamma-1}}
$$

where $P_{r}$ is ratio of the tank pressure to atmospheric pressure. After this criterion is reached, the discharge rate is given by

$$
Q=C_{o} A_{h}\left[P_{t} \rho_{g}\left(\frac{2 \gamma}{\gamma-1}\right)\left(P_{r}^{-\frac{2}{\gamma}}-P_{r}^{-\frac{\gamma+1}{\gamma}}\right)\right]^{\frac{1}{2}} .
$$

Calculations are conducted iteratively by adjusting the tank pressure and density at each time step with the isentropic assumption. 


\section{Liquefied Gas Releases}

Liquefied gas releases are treated by first calculating the liquid release rate from the container given by Eq. 3.7. As discussed previously, this material either flashes to vapor, is aerosolized and entrained with the vapor, or remains liquid and falls to the ground. The flash fraction $f$ is given by

$$
f=\frac{c_{p}\left(T_{t}-T_{b}\right)}{h_{f g}},
$$

where $T_{t}$ is the tank temperature and $T_{b}$ is the normal boiling point. This fraction of remaining material that is aerosolized and entrained into the flashed vapor is calculated using empirical relationships based on the discharge kinetic energy of the two-phase mixture provided by Johnson and Woodward (1999). Released material that does not flash to vapor or become entrained in the vapor clouds is deposited on the ground. Evaporation of the remaining material is then estimated using the pool evaporation algorithms.

\subsubsection{Water-Reactive Materials}

For water-reactive materials, a different method is needed to estimate $\mathrm{TIH}$ emission rates to the atmosphere. Because of the myriad of water body types, reaction scenarios, and release mechanisms; the general lack of historical data on water entry releases; the inadequate characterizations of water body turbulence and mixing; and the lack of experimental data to validate sophisticated water reactivity models, treatment of all possible incident scenarios is not possible. It was therefore necessary to develop a simplified general approach for assessing the level of public protection required for each spill. This effort was started in the ERG1996 analysis and supplemented with an experimental program begun in the ERG2000 analysis to provide quantitative data. We added experiments on many more materials for the ERG2004.

The basic formula employed to compute the release rate of TIHWR gases for most materials assumes the reaction rate exponentially decreases with time such that

$$
Q(t)=M_{o} f_{s} \beta \lambda e^{-\lambda t}
$$

where

$$
\begin{aligned}
& Q(t)=\text { time dependent release rate of TIHWR product }(\mathrm{kg} / \mathrm{s}), \\
& M_{O}=\text { initial mass of parent chemical released into water }(\mathrm{kg}), \\
& f_{S}=\text { maximum stoichiometric yield }(\mathrm{kg} \text { TIHWR product } / \mathrm{kg} \text { parent chemical), }
\end{aligned}
$$




$$
\begin{aligned}
& \beta=\text { efficiency factor for the reaction }(0 \leq \beta \leq 1), \text { and } \\
& \lambda=\text { first-order rate coefficient (per second). }
\end{aligned}
$$

In the CASRAM source model, Eq. 3.17 is discretized to yield values for $\Delta Q$ for each parcel of a parent chemical $\Delta M_{o}$ released into a water body. The time dependent total evolution rate of TIH product(s) from the spill is then the sum of the individual releases arising from each parcel, where the time in Eq. 3.17 is that elapsed since each parcel entered the water.

The key empirical parameters necessary to apply Equation 3.17 are $\beta$ and $\lambda$, since the stoichiometric yield is easily calculated. Experimental data for $\beta$ and $\lambda$ are not available in the chemical literature. In our past efforts, extensive search of the chemical literature yielded only qualitative descriptions of water reactivity for most of the TIHWR materials identified over the previous decade of ERG analyses. Such descriptions allow only a crude estimate of $\beta$ and $\lambda$, accurate to a factor of 2 at best. Moreover, troubling inconsistencies in these qualitative descriptions were sometimes found in different literature sources. In several cases, the descriptions disagreed with the direct experience of at least one of the authors. Furthermore, we could not find any examples in which the deliberate release of a bulk chemical into a relatively large amount of water had been followed by the measurement of TIHWR production amounts.

To help provide a quantitative basis to the TIHWR analysis, we conducted a series of direct experiments on about 50 water reactive chemicals beginning in 1999 (for the ERG2000 analysis). These experiments were small in scale, leading to some substantial issues in trying to scale the results up to the size of transportation spills. Nevertheless, the experiments greatly increased the accuracy of estimates for $\beta$ and also provided a direct measure of $\lambda$, which is even more difficult to estimate from the qualitative descriptions.

In recent experiments, we found that Eq. 3.17 was not adequate to fully describe the reaction for a narrow class of silanes. For 5 of these materials in our experimental series, there appeared to be an initial induction period characterized by a constant release rate such that

$$
Q(t)=M_{o} \frac{m_{i}}{T_{\text {ind }}}, \quad\left(t \leq T_{\text {ind }}\right)
$$

where $m_{i}$ is an initial production coefficient and $T_{\text {ind }}$ is the initial induction period. For these materials, the gas evolution starts slowly and then greatly accelerates after the initial induction period, indicating the reaction is likely autocatalytic. For times after $T_{\text {ind }}$, the reaction proceeds at an exponential rate and can be modeled using Eq. 3.17 with some minor modifications in $\beta$ as discussed in Appendix D.

A review of literature on TIHWR chemicals showed that only two water body characteristics seemed likely to influence their reactions in water: acidity and temperature. For a few chemicals, the presence of acidity was reported to increase the speed and degree of the reaction. However, the characterization of these effects is not well developed. Because of this, in addition to the difficulty of obtaining water acidity data for natural water bodies, we do not attempt to account for water acidity variations in the ERG analysis. The effect of temperature is 
substantially stronger than acidity, however, and simpler to treat in a quantitative fashion. The reaction rate of many materials roughly doubles for every $10^{\circ} \mathrm{C}$ increase in temperature. Fortunately, the temperature of natural water bodies is well characterized by a network of water quality stations and buoys, as discussed in Section 3.2.3. The temperature dependence of the primary rate constant $\lambda$ was included in the analysis via the Arrhenius equation, written as

$$
\lambda=\lambda_{o} \exp \left[-6610\left(\frac{1}{T_{w}}-\frac{1}{T_{o}}\right)\right],
$$

where $T_{O}$ is a reference temperature $\left(20^{\circ} \mathrm{C}\right.$ for our analysis) and $T_{W}$ is water temperature determined by the procedures outlined in Section 3.2.3. The chemical-specific constant $\lambda_{\mathrm{o}}$ was determined experimentally. In applying this relationship, the rate constant $\lambda$ for a given hypothetical spill was specified by finding $T_{W}$ on the Julian day of the simulated accident at the nearest WQN or GLB station. Then the Arrhenius formula was applied to the rate constant $\lambda_{\mathrm{o}}$ in Table D.1 in Appendix D.

\subsection{ANALYSIS OF ATMOSPHERIC DISPERSION}

In the atmospheric dispersion modeling phase of the problem, the hazardous material release rate and meteorology are used to estimate chemical concentrations downwind of the release. When plume buoyancy is insignificant (i.e., passive dispersion), downwind concentrations are linearly related to (1) the release rate in the event of a continuous release or (2) the release amount in the event of an instantaneous release. The dispersion of the material is strongly dependent on the meteorology. In relative terms, dispersion is very good during daytime with no cloud cover (i.e., maximum surface heating) and very poor during nighttime with clear skies and light winds. Given the same emission rate, ground-level material concentrations downwind of a near-surface release can vary by three orders of magnitude between these two extremes. For moderate-to-high wind speeds and/or overcast conditions, atmospheric dispersion falls between these two limiting cases.

The dispersion method used in the ERG2004 is very similar to that employed in preparation of the ERG2000, with the only differences being minor adjustments to the horizontal dispersion coefficients. For passive dispersion, we use the vertical dispersion model of Brown (1997), which is applicable for calculating ground-level concentrations from near-ground releases. For heavier-than-air, or so-called dense gas, releases, we use relationships from the DEGADIS model. The dense gas model is employed for liquefied gas releases in which cooling of the plume and aerosol entrainment increase the plume density to the point where the passive dispersion assumption is no longer valid. For many liquefied gases, especially those that are highly toxic, the use of a dense gas dispersion model in the initial phases of the plume trajectory calculation does not have a significant impact on the final PAD estimates. For a narrow class of less toxic liquefied gases such as ammonia, incorporation of a dense gas model does have a more substantial effect. 
We first discuss some general concepts about atmospheric dispersion modeling of hazardous materials releases in Section 3.4.1, and then describe the CASRAM dispersion model as applied to both passive and dense-gas releases.

\subsubsection{Overview of Atmospheric Dispersion}

\subsubsection{Diurnal Aspects of Plume Dispersion}

The physical processes governing the development and maintenance of the atmospheric boundary layer (ABL) are very different during day and night, leading to boundary layers with extremely different sizes and characters. These marked differences substantially influence the ability of the ABL to disperse pollutants released near the ground, thereby giving rise to the pronounced differences in downwind concentrations and therefore in PAD values appearing in the Table. This section briefly describes the characteristics that distinguish the daytime and nighttime ABLs and elucidates how these variations lead to the differences in PAD values between the two cases.

The unstable or convective boundary layer (CBL), typical of daytime conditions, is shown in Figure 3.8, panel a. The boundary layer structure is a result of the surface heating caused by solar radiation. This heating destabilizes the lower layers of the ABL, producing large convective cells. These convective cells extend vertically to the lowest temperature inversion, and they efficiently transport heat, momentum, and any material contaminants released into the atmosphere. As the day progresses, the temperature inversion marking the top of the boundary layer rises from near the ground to between 0.5 and $4 \mathrm{~km}$ above the surface as a result of the entrainment of stably stratified air above the inversion into the cooler air of the boundary layer below.

An example of a dispersing plume in a typical CBL is pictured in Figure 3.8, panel b. Experimental observations, most notably those of Willis and Deardorff (1976), have indicated that vigorous convection, which typifies the CBL, results in a "rising centerline" phenomenon. In this process, energetic thermals lift the plume or cloud off the ground faster than it disperses downward. This consequence of dispersion in a fully convective boundary layer is primarily responsible for the rapid decay of material concentrations downwind from ground-level sources when the release occurs in the middle of the day.

In the absence of solar heating, the nighttime ABL structure is driven by cooling at the surface as heat is radiated to space. This situation leads to the development of a stably stratified boundary layer (SBL), which is typically much shallower and less energetic than its unstable counterpart, as illustrated in Figure 3.9, panel a. The surface cooling strongly stratifies the lowlevel air, thereby creating a tenuous balance between the turbulence produced by wind shear and turbulent energy dissipation. The rapid dissipation of turbulent energy greatly limits the vertical eddy size and restricts vertical mixing. 

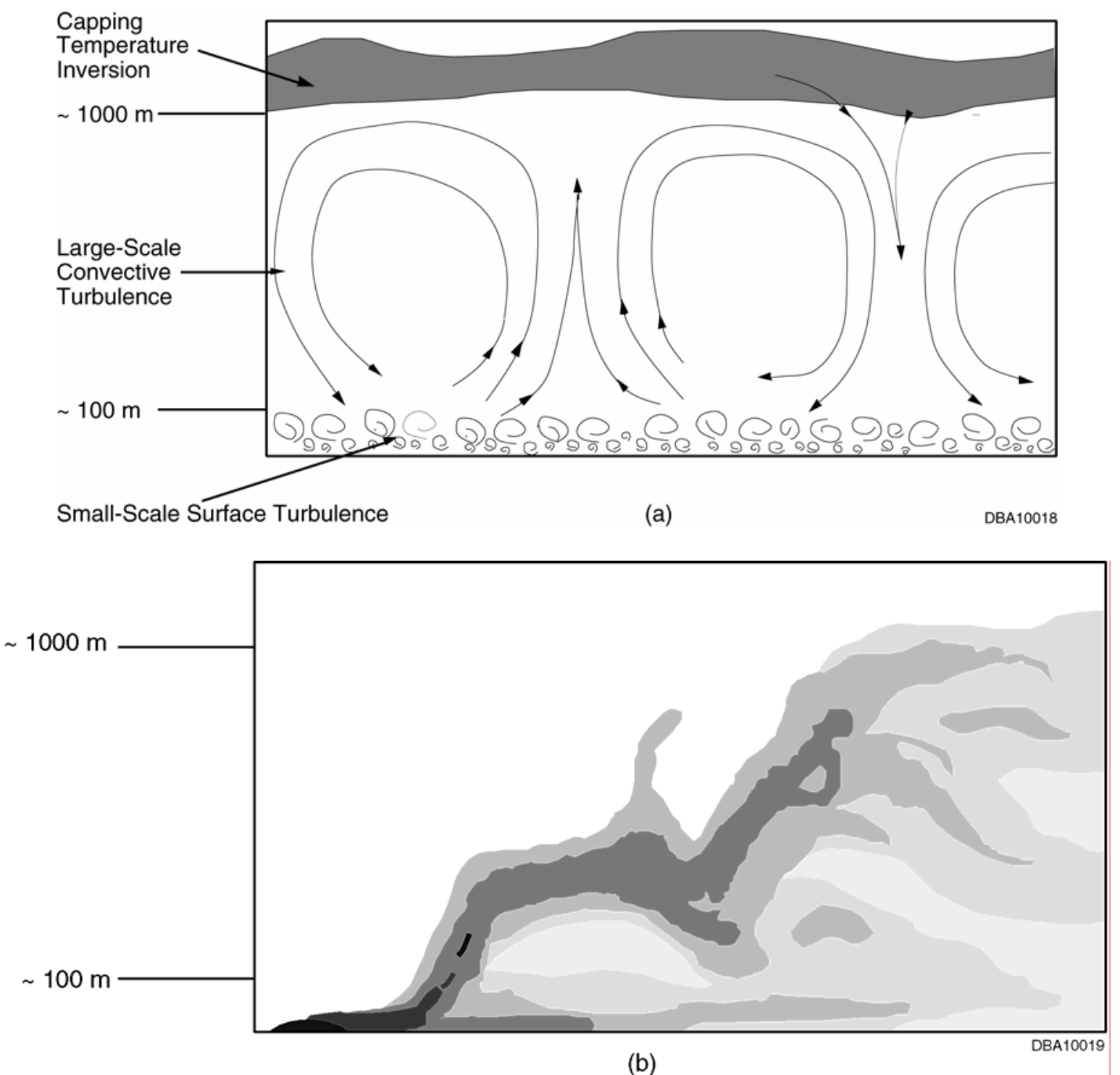

FIGURE 3.8 Typical Daytime Convective Boundary Layer Showing (a) an Instantaneous Wind Field and (b) an Instantaneous Material Concentration Field from a Ground-Level Release (characteristic height of about $1,000 \mathrm{~m}$ for the boundary layer and about $100 \mathrm{~m}$ for the surface layer provided for reference)

Dispersion in a typical SBL is pictured in Figure 3.9, panel b. The vertical stability and shallow depth profoundly affect dispersion processes by confining material contaminants to a thin layer near the ground. In very stable conditions, such as those represented in the $90^{\text {th }}$-percentile level of safe distance distributions, the SBL is usually less than $100 \mathrm{~m}$ deep. When a contaminant is released into the SBL, it diffuses to its maximum vertical extent in a relatively a short distance, forcing most of the dispersion to occur two-dimensionally along the ground. This plume confinement allows comparatively high concentrations to be observed near the surface at considerable distances from the release site, leading to substantially longer PADs for daytime incidents. 


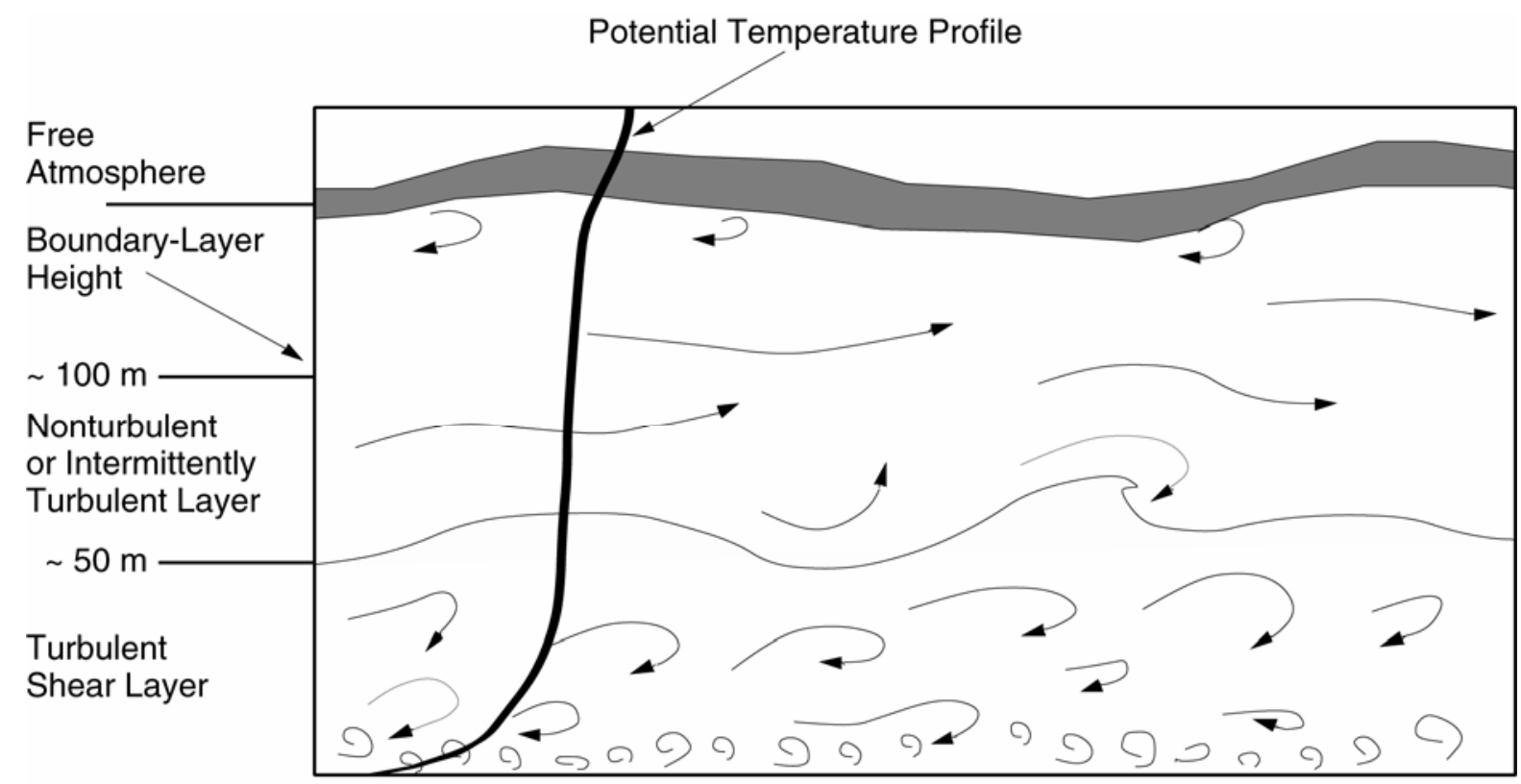

(a)

DBA10020

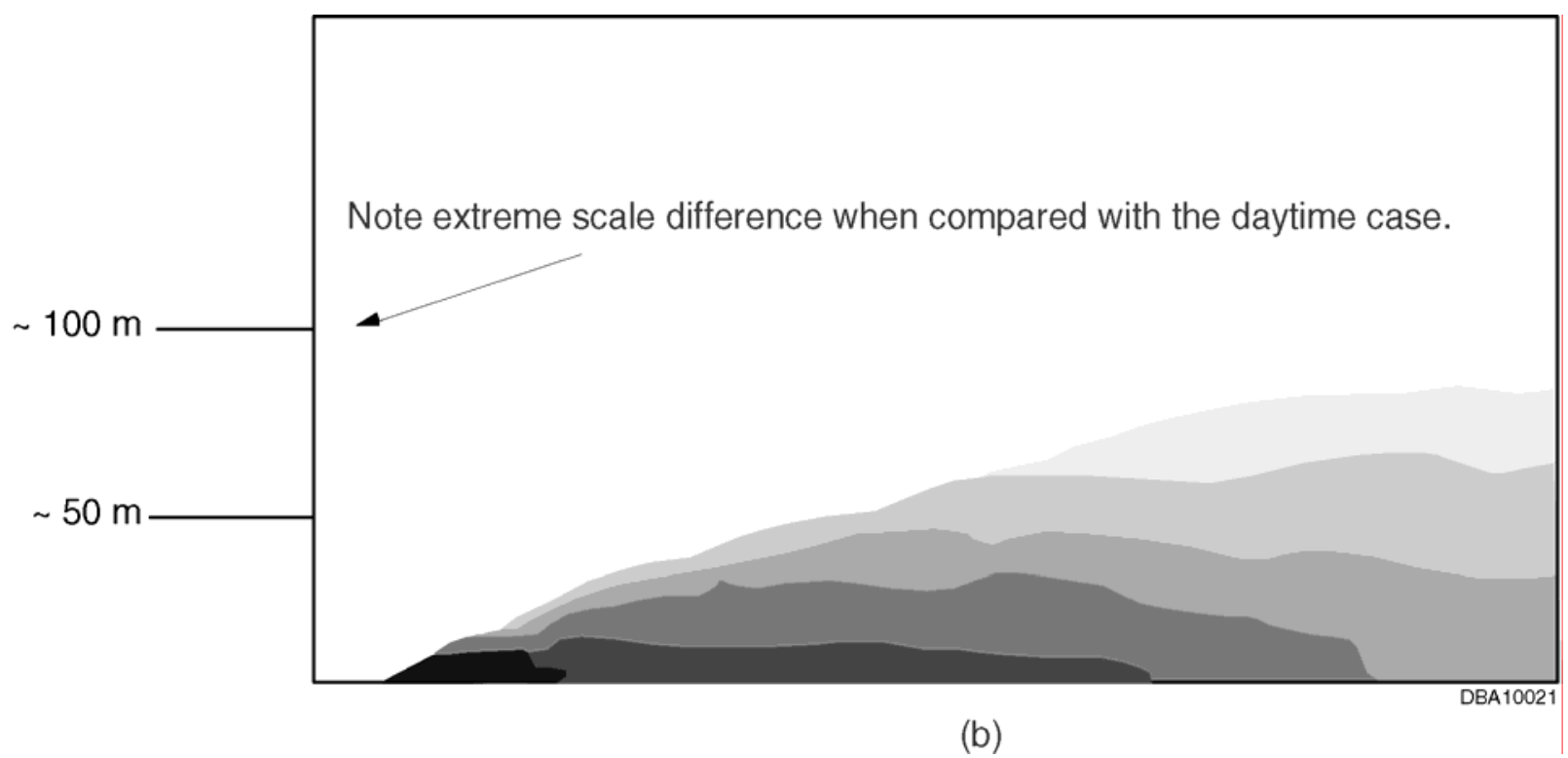

FIGURE 3.9 Typical Nighttime Stable Boundary Layer Showing (a) an Instantaneous Wind Field and (b) an Instantaneous Material Concentration Field from a Ground-Level Release (characteristic height of about $50 \mathrm{~m}$ for the surface shear layer and about $100 \mathrm{~m}$ for the boundary layer provided for reference) 


\subsubsection{Effects of Dense Gas on Plume Dispersion}

The discussion of atmospheric dispersion to this point has been confined to passive dispersion (i.e., cases in which the density of the ambient plume does not affect its dispersion). However, in many cases of practical interest, the effect of a high density (relative to air) of the hazardous chemical discharge becomes important in considering impacts within 1-2 km of the release point. So-called dense gas effects result not only from the properties of the material released but also from the methods of storage and the conditions of the release. Most cases of interest have focused on combustibles or toxic compounds that have boiling points below ambient temperature. These compounds are commonly transported or stored as liquids and then maintained in liquid phase (1) at or near their saturation temperature at atmospheric pressure by refrigeration and insulation (i.e., refrigerated liquid) or (2) at ambient temperature by pressurization (i.e., pressurized liquid or liquefied gas). For transportation incidents, cases that could lead to significant dense gas effects fall into one or more of the following broad categories:

- Chemicals with a high molecular weight when compared with air (e.g., chlorine, arsine) coupled with a large release quantity or high release rate (i.e., greater than $1 \mathrm{~kg} / \mathrm{s}$ );

- Refrigerated chemicals with a relatively low molecular weight when their temperature upon release is cold relative to the ambient temperature (e.g., a cold methane release evolving from the boiling of refrigerated liquefied natural gas);

- Pressurized liquids containing chemicals with a low to moderate molecular weight that, although they are less dense than air at their boiling point, cool rapidly and entrain aerosol generated in the release process, thereby creating a denser-than-air plume; and

- Chemicals that undergo molecular association (hydrogen fluoride) and/or transform to secondary hazardous compounds because of their ambient watervapor reactivity (e.g., sulfur trioxide/oleum and nitrogen tetroxide). (These are not necessarily the same group of compounds that are water-reactive as defined in this report.)

Several major effects can be observed during the dispersion of a ground-level, dense gas cloud that do not occur during the dispersion of a neutrally buoyant cloud. One is that there is much less vertical turbulent mixing between the dense gas cloud and the ambient atmosphere because of the strong stable density stratification of the cloud relative to the surrounding ambient air. Another is the presence of gravity-induced flow resulting from horizontal density gradients. These two effects result in a much shallower and wider cloud than a cloud that results from an analogous neutral density release. In addition, the movement of the dense gas cloud on uneven terrain can follow a downhill slope independent of the wind direction, and the cloud can become trapped in valleys or low spots. The magnitude of these dense gas effects depends on the size of the release, local meteorological conditions, and the physical properties of the chemical released. 
As the dense plume travels downwind, the plume warms through ambient heating (e.g., from sunlight, ground heating, etc.) and entrainment of warmer air. At some downwind distance, the density of the plume will decrease to the point where the density can no longer retard the vertical dispersion of the plume by ambient turbulence, and the plume will subsequently disperse as a passive cloud.

\subsubsection{CASRAM Dispersion Model}

\subsubsection{Passive Dispersion}

Like many other dispersion models, CASRAM separates the dispersion calculation into two components: horizontal dispersion and vertical dispersion. In CASRAM, vertical turbulent dispersion is treated with a Lagrangian-integral model parameterized in terms of mean plume height, average advection velocity, and a dimensionless travel time. These parameters are expressed as integral equations written in terms of plume travel time and atmospheric boundary layer parameters. Continuous releases are treated as plumes, and instantaneous releases are treated as puffs. Horizontal turbulent dispersion is represented via Gaussian relationships that are parameterized in terms of the Lagrangian time scale and lateral wind direction fluctuations. Plume calculations are straight line in nature, since terrain effects are not currently considered in CASRAM.

The concentration relationship for continuous plume releases from point sources is represented in terms of the crosswind-integrated concentration (CWIC), chemical mass release rate $Q$, and a traditional Gaussian expression for the horizontal plume spread. The ground-level concentration distribution is represented as follows:

$$
C(x, y, z=0)=\frac{Q \hat{C}_{y}(x, z=0)}{\sqrt{2 \pi} \sigma_{y}} \exp \left[-\left(\frac{y}{\sqrt{2} \sigma_{y}}\right)^{2}\right]
$$

where

$$
\begin{aligned}
& \hat{C}_{y}=\text { CWIC normalized by the release rate, } \\
& \sigma_{y}=\text { lateral plume spread, and } \\
& y=\text { lateral distance from the plume centerline. }
\end{aligned}
$$


For releases with finite width, corresponding to large pool releases or dense gas releases as they become passive, the following expression is used:

$$
C(x, y, z=0)=Q \hat{C}_{y}(x, z=0)\left(\operatorname{erf}\left[\frac{y_{o}-y}{\sqrt{2} \sigma_{y}}\right]+\operatorname{erf}\left[\frac{y_{o}+y}{\sqrt{2} \sigma_{y}}\right]\right) .
$$

Peak concentration averages resulting from instantaneous releases are calculated according to the relationship

$$
C(x, y, z=0)=\frac{M \hat{C}_{y}(x, z=0)}{\sqrt{2 \pi} \sigma_{y} T} \operatorname{erf}\left[\frac{\mathrm{UT}}{2 \sqrt{2} \sigma_{y}}\right] \exp \left\lfloor-\left(\frac{y}{\sqrt{2} \sigma_{y}}\right)^{2}\right\rfloor,
$$

where

$$
\begin{aligned}
& M=\text { total release amount } \\
& T=\text { averaging time, and } \\
& U=10-\mathrm{m} \text { wind speed. }
\end{aligned}
$$

The subsections below present the methodology for determining vertical dispersion (i.e., for determining $C_{y}$ as a function of downwind distance) and then discuss the determination of $\sigma_{y}$, thus providing horizontal dispersion.

\section{Vertical Dispersion}

The vertical dispersion model in CASRAM is a natural extension of the surface-layer, similarity-based model of van Ulden (1978). The van Ulden approach centers around an exact

solution of the advection-diffusion equation. When written in terms of the CWIC form and neglecting stream-wise diffusion, it reads as follows:

$$
U(z) \frac{\partial C_{y}}{\partial z}=\frac{\partial}{\partial z}\left[K_{z}(z) \frac{\partial C_{y}}{\partial z}\right]
$$

where $K_{z}$ is the vertical diffusivity for a scalar. The solution van Ulden advances is written in terms of the mean plume height $\bar{z}$, the average plume advection velocity $\overline{U_{p}}$, and the power-law wind speed coefficient $m$ and diffusivity coefficient $n$. The coefficients $m$ and $n$ are defined such that

$$
U(z)=u_{o} z^{m}
$$

and 


$$
K_{z}(z)=k_{o} z^{n}
$$

where $u_{o}$ and $k_{o}$ are constants representing the wind speed and diffusivity at $1 \mathrm{~m}$. The advectiondiffusion equation subject to the above power-law relationships yields an exact solution (see Roberts 1923; Calder 1949). The work of van Ulden (1978) extends this analysis by using the power-law coefficients to determine the concentration profile shape a priori (from the Roberts analysis) and then formulates integral relationships for the mean plume height and average advection velocity in terms of the more physically correct similarity functions for wind speed and diffusivity. The van Ulden (1978) solution reads:

$$
\hat{C}_{y}(x, z)=\frac{A}{\bar{z} \bar{U}_{p}} \exp \left[-\left(\frac{B z}{\bar{z}}\right)^{s}\right],
$$

where

$$
\begin{aligned}
& A=\frac{s \Gamma(2 / s)}{\Gamma(1 / s)^{2}}, \\
& B=\frac{\Gamma(2 / s)}{\Gamma(1 / s)},
\end{aligned}
$$

and $s$ is a shape parameter given by $1-m-n$. In Eqs. 3.27 and 3.28, $\Gamma$ represents the Gamma function.

In applying this model, van Ulden (1978) parameterized the mean plume height and average advection velocity using surface-layer similarity functions for wind speed and diffusivity. In a subsequent study, Gryning et al. (1983) extended this analysis by adding a sophisticated relationship to determine the shape parameter $s$ as a function of stability and downwind distance. The relationships for $s$ were determined through analysis of numerical solutions for the advection-diffusion equation for a wide range of conditions. In a detailed model-data comparison that used CWIC data from the Prairie Grass experiments (Barad 1958), Gyning and colleagues demonstrated the utility of the Lagrangian empirical model in representing the concentration profile as well as in estimating ground-level concentrations. This model provided an excellent description of dispersion in the surface layer. However, its application is limited to dispersion problems where the plume is confined to the surface layer. When the plume rises above the surface layer, the concentration profile given by Eq. 3.26 - as well as the similarity relationships for $\bar{z}$ and $\overline{U_{p}}$ - lose validity. For problems of practical interest, this rather severe constraint strongly limits the applicability of Eq. 3.26.

Brown (1997) modified the original van Ulden work to correct this limitation. In this revised formulation, the normalized ground-level concentration is expressed as 


$$
\hat{C}_{y}(x, z=0)=\frac{A^{\prime}(\tilde{s}) F\left(x ; u_{*}, z_{i}, H_{s}\right)}{z_{u} \overline{U_{p}}}
$$

where $z_{u}$ is defined such that

$$
U\left(z_{u}\right)=\overline{U_{p}}
$$

and

$$
A^{\prime}(\tilde{s})=\frac{1.6 \tilde{s} \Gamma(2 / \tilde{s})}{\Gamma(1 / \tilde{s})^{2}}
$$

Here, $A^{\prime}$ takes the same functional form as $A$ in van Ulden's model, except that it is multiplied by 1.6 to account for the ratio of $\bar{z}$ to $z_{u}$ and depends on a modified shape parameter, $\widetilde{s}$.

The main departure of this approach from the models of van Ulden (1978) and Gryning et al. (1983) is in the boundary-layer function $F$. This empirical function is added to treat dispersion within the greater ABL outside the surface layer. As previously noted, the van Ulden model was developed using the quasi-exponential concentration profile. For surface-layer dispersion, this assumed form for the concentration profile adequately represents the concentration profiles observed in field studies, most notably the Prairie Grass experiments. However, once the plume is influenced by boundary-layer effects outside of the surface layer, the concentration profiles depart significantly from the exponential form. In unstable conditions, this departure is particularly pronounced, since the plume centerline can actually lift off the ground, creating a maximum concentration aloft. In stable conditions, the opposite effect is observed, in which the concentration profile is flattened as a result of the rapid decrease in turbulent energy with height. The transition function $F$ allows us to adapt this methodology, which was originally developed to model surface-layer dispersion, to treat dispersion throughout the entire ABL.

Brown (1997) developed relationships for $z_{u}, \widetilde{s}$, and $F$ through a parametric analysis of a Langevin-equation Monte Carlo dispersion model validated with data from field and laboratory experiments. In applying the CASRAM vertical dispersion model, $\widetilde{s}$ is a function of meteorology and therefore does not vary with distance and $F$ is represented as a function of travel time and meteorology. The heart of the calculation lies in $z_{u}$, which is calculated using integral relationships represented as a function of travel time. Numerical integration of this relationship provides $z_{u}$ as a function of downwind distance, which, together with $\widetilde{s}$ and $F$, allows the evaluation of Eq. 3.24 and ultimately Eq. 3.20, 3.21, or 3.22. 


\section{Horizontal Dispersion}

Under horizontally homogeneous conditions, the Gaussian model correctly represents lateral concentration distributions. In CASRAM, the lateral spread from nonbuoyant releases can be related to the standard deviation of the horizontal wind fluctuations and is determined using relationships originally proposed by Draxler (1976). Here, $\sigma_{y}$ is given by

$$
\sigma_{y}=\sigma_{v} f_{y}\left(\frac{t}{T_{d}}\right),
$$

where

$$
\begin{aligned}
& \sigma_{v}=\text { standard deviation of transverse velocity, } \\
& t=\text { transit time (estimated using wind speed at } 3 \mathrm{~m} \text { and distance), } \\
& T_{d}=\text { dispersion time scale related to the Lagrangian time scale, and } \\
& f_{y}=\text { a nondimensional function of travel time. }
\end{aligned}
$$

The empirical form of $f_{y}$ is estimated through evaluating field data. Several forms of $f_{y}$ have been proposed (Irwin 1983; Gryning and Lyck 1984), but the empirical expression that provides the best overall fit with available field data is the form proposed by Draxler (1976), given by

$$
f_{y}=\frac{1}{1+0.9 \sqrt{t / T_{d}}}
$$

For surface releases, a $T_{d}$ of $300 \mathrm{~s}$ is used for unstable conditions and a maximum of $300 \mathrm{~s}$ or $0.001 t^{2} \mathrm{~s}$ is used for stable conditions. The appeal of Draxler's relation is that it is developed from diffusion data from many experiments and that it is consistent with Taylor's limit for both small $\left(\sigma_{y} \sim t\right)$ and large $\left(\sigma_{y} \sim t^{1 / 2}\right)$ travel times (Taylor 1921).

Equation 3.32 requires the standard deviation of transverse velocity $\sigma_{v}$. In the absence of observational data, $\sigma_{v}$ is determined with the interpolation equation of Panofsky et al. (1977)

$$
\sigma_{v}=u_{*}\left(12-0.5 \frac{Z_{i}}{L}\right)^{1 / 3}
$$

during unstable conditions and the proportionality relation of Panofsky and Dutton (1984)

$$
\sigma_{v}=1.92 u_{*}
$$

during stable conditions. 
In very stable conditions, Eq. 3.35 fails to adequately represent $\sigma_{v}$, since wind speed and friction velocity become very small. For instance, if the friction velocity is about $0.015 \mathrm{~m} / \mathrm{s}$, indicative of a wind speed of about $0.5 \mathrm{~m} / \mathrm{s}$ with $z_{O}=0.1 \mathrm{~m}$ on a clear night, Equation 3.35 predicts $\sigma_{v}=0.03 \mathrm{~m} / \mathrm{s}$. One kilometer from the source, this value of $\sigma_{v}$ yields $\sigma_{y}=36 \mathrm{~m}$ from Eq. 3.32. Considering the 2,000-s travel time, such a narrow plume width at ground level is not realistic when the meandering nature of stable boundary layer flows is considered. To overcome this problem, we assign a minimum value of $0.15 \mathrm{~m} / \mathrm{s}$ for $\sigma_{v}$ on the basis of observations presented by Hanna and Chang (1992).

\subsubsection{Dense Gas Dispersion}

A dense gas algorithm was added to the CASRAM methodology as part of the ERG2000 study after a detailed review of available dense gas models. The review identified five dense gas models that were well documented and would be applicable within the CASRAM framework: DEGADIS (Havens 1988), HEGADAS (Post 1994), SLAB (Ermak 1990), SCIPUFF (Defense Threat Reduction Agency 1999), and TSCREEN (U.S. Environmental Protection Agency [EPA] 1992). Based on many considerations, most importantly accuracy and ease of incorporation into the CASRAM framework, we opted to rely on the empirical entrainment parameterizations within the DEGADIS formulation for the CASRAM heavy gas dispersion model, although future modifications to the CASRAM dense gas dispersion model may involve incorporation of the HEGADAS formulation since it was being revised when this work was completed.

Like the passive gas dispersion model used in CASRAM, the dense gas algorithm is integral in nature. The initial conditions are specified by the vapor release rate $Q_{v}$ and aerosol release rate $Q_{a}$ from the container, which are calculated from the CASRAM emission rate model. The first step is evaluating the volumetric flow rate of vapor exiting the container $\dot{V}_{s v}$, which is given by

$$
\dot{V}_{s v}=\frac{Q_{v}}{\rho_{v}}
$$

where $\rho_{\nu}$ is the density of the chemical vapor at the boiling point. The initial volumetric flow rate of the cloud $\dot{V}_{c i}$ is taken as

$$
\dot{V}_{c i}=\left(1+\beta_{e i}\right) \dot{V}_{s v}
$$

where $\beta_{e i}$ is the entrainment parameter at the source, taken as 2 . This is a rough estimate that depends on many factors. However, the model results at distances more than $10-20 \mathrm{~m}$ from the source are not sensitive to $\beta_{e i}$.

The initial cloud is assumed to have a flat, cylindrical shape (rectangular in two dimensions) with width $2 r_{i}$ and height $h_{i}$, where $r_{i}=h_{i}$. To estimate the cloud dimensions from the volumetric flow rate, one must solve the relationship 


$$
h_{c i}=\frac{\dot{V}_{c i}}{2 \overline{U_{p}} r_{i}},
$$

where

$$
\overline{U_{p}}=u\left(z=0.6 h_{c i}\right) .
$$

The solution of Eqs. 3.38 and 3.39 is necessarily iterative and subject to the constraint that $h_{c i}$ is more than $1.5 \mathrm{~m}$.

The initial cloud density is then defined in terms of the sum of the mass flow rates of the flashed vapor and aerosol plus the entrained air as

$$
\rho_{c i}=\frac{Q_{v}+Q_{a}+\beta_{e i} \dot{V}_{v} \rho_{a}}{\dot{V}_{c i}}
$$

where the last term on the right side of the numerator accounts for the mass of air initially entrained into the plume.

After release, the cloud spreads horizontally under the influence of its negative buoyancy while it grows vertically through entrainment of air from above. Note that dense gas plumes are marked by a much higher horizontal growth rate than vertical growth rate. The horizontal gravity spread is assumed to depend on the cloud advection speed and Richardson number for continuous releases and is computed in a form equivalent to the model of Raj (1985) such that

$$
\frac{d r}{d x}=\frac{\beta_{e} u_{*}}{\overline{U_{p}}} \sqrt{R i_{*}}
$$

Here, $\beta_{e}$ is an entrainment parameter, taken as 1.15 , and $R i_{*}$ is the local cloud Richardson number given by

$$
R i_{*}=\frac{g h_{c}\left(\rho_{c}-\rho_{a}\right)}{\rho_{a} u_{*}^{2}}
$$

Vertical cloud growth is governed by vertical entrainment of air into the cloud, which can be conveniently defined in terms of cloud advection speed and entrainment velocity $v_{e}$ in the integral equation

$$
\frac{d h_{c}}{d x}=\frac{v_{e}}{\overline{U_{p}}},
$$


where the vertical entrainment velocity is as used in the DEGADIS model, namely

$$
v_{e}=\frac{0.4 u_{*}}{0.88+0.099 R i_{*}^{1.04}} .
$$

Equations 3.41 and 3.43 make up a coupled set of differential equations that are solved at successive points downwind of the source. The solution proceeds until the critical Richardson number $R i$ is less than 50 . This critical Richardson number is defined differently than the local cloud Richardson number and is given by

$$
R i_{c}=\frac{U\left(h_{c}\right)\left(\rho_{c}-\rho_{a}\right) g h_{c}}{\rho_{a} u_{*}^{3}}
$$

After this Richardson number criterion is met, we initialize the passive gas dispersion model by matching the cloud height and assuming a uniform concentration across the width of the cloud of $2 r$. Therefore, the parameter $y_{0}$ in Eq. 3.21 is set to $r$. 


\section{HEALTH CRITERIA}

Over the past 15 years, various health criteria have been used to develop Initial Isolation Zones and PADs. Early efforts employed occupational exposure guidelines, such as threshold limit values (TLVs) established by the American Conference of Governmental Industrial Hygienists (ACGIH). Beginning in 1990, the ERPGs developed by the AIHA were identified as the best available health criteria for evaluating the health significance of accidental releases and hence formed the basis for defining PADs. This preference was based on a number of factors, including the high quality of documentation, the consensus approach with which the values are derived, and the consideration of exposure to the general population. In the last few years, Acute Exposure Guidance Levels (AEGLs), which are defined similar to ERPGs but for a variety of exposure times, have emerged on the scene.

In 1995, as part of a continuing effort to improve the ERG, an analysis of the various health criteria used to develop PADs was performed because ERPGs were only available for a limited number of chemicals on the TIH list. This analysis indicated that certain health criteria were stratified when compared with ERPG-2 values. This stratification had a significant influence on the PADs calculated with these health criteria. As a result, efforts were undertaken to further identify and minimize sources of stratification. These efforts are described in Section 4.1 and included: (1) reviewing published studies on health criteria used to evaluate accidental releases, (2) performing an independent analysis of the ERPG values, and (3) convening an independent panel of expert toxicologists to recommend uses for health criteria and ways to minimize stratification. This effort led to the development of a solid peer-reviewed framework for specifying health criteria for PAD estimation and was first used in the ERG1996.

For the ERG2000 and ERG2004 analyses, we left this basic framework unchanged. The limited changes included incorporating several new ERPGs that became available between 1996 and 2003. In addition, we studied several new materials that had been added to the TIH list to determine appropriate health criteria. We also performed an extensive literature search to determine if any new acute toxicity information was published for the materials on the TIH list in the past several years that would be relevant to our analysis. Except for the ERPG values noted above, however, no new studies relevant to our analysis were identified.

As part of the ERG2004 analysis, we also began considering the issues involved with incorporating the new AEGLs developed by the Federal Advisory Committee, a collaborative effort of the public and private sectors. Since few final AEGLs were available when the ERG2004 was prepared, we decided to postpone the addition of AEGLs to the ERG methodology until work commences on the next edition of the ERG (expected to be published in 2008). The potential issues involved with their inclusion are discussed in Section 4.4. 


\subsection{REVIEW OF PAST DEVELOPMENT EFFORTS}

\subsubsection{ERPGs}

Emergency Response Planning Guideline (ERPG) concentrations, in particular the ERPG-2, have been the basis for estimating PADs in the ERG since 1990. ERPGs are developed by the American Industrial Hygiene Association through a rigorous peer-review process that emphasizes human experience to the extent that such information is available. However, since human exposure data are rarely available except for low-level exposures, animal exposure data, generally from acute inhalation toxicity studies, are most often employed as the basis for the ERPG concentrations. ERPGs are given for three levels:

- ERPG-3 - The maximum airborne concentration below which it is believed that nearly all individuals could be exposed for up to $1 \mathrm{~h}$ without experiencing or developing life-threatening health effects.

- ERPG-2 - The maximum airborne concentration below which it is believed nearly all individuals could be exposed for up to $1 \mathrm{~h}$ without experiencing or developing irreversible or other serious health effects, or symptoms that could impair an individual's ability to take protective action.

- ERPG-1 - The maximum airborne concentration below which it is believed nearly all individuals could be exposed for up to $1 \mathrm{~h}$ without experiencing other than mild, transient adverse health effects or perceiving a clearly defined objectionable odor.

As of July 2004, the AIHA has published ERPG concentrations for 111 different chemicals. Typically the AIHA adds about 7-10 chemicals per year to their list and modifies previously published values for another 7-10 materials.

\subsubsection{Comparisons of ERPGs with other Health Criteria}

A number of published studies compare alternative health criteria for use in evaluating accidental releases. These studies have helped to resolve several questions on the relationship between ERPGs and alternative values, which include NIOSH IDLH levels, National Research Council Emergency Exposure Guidance Levels (EEGLs), and occupational health guidelines.

Craig et al. (1995) calculated the ratios of ERPG-2 values to other health criteria and examined the statistical relationship between them. The mean, coefficient of variation, and coefficient of determination of these ratios were calculated. The analysis included ERPG data for 35 chemicals. None of the existing values adequately estimated ERPG-3 values. For example, National Research Council 30-min EEGLs substantially underestimated ERPG-3 values (mean ratio $=0.55)$, while NIOSH IDLH values overestimated ERPG-2 values (mean ratio $=2.48$ ). 
A good correlation was observed between ERPG-2 values and National Research Council 60-min EEGLs and EPA levels of concern (LOCs) (mean ratios of 0.99 and 0.82, respectively). Similarly, good correlations were observed between ERPG-2 and Occupational Safety and Health Administration (OSHA) permissible exposure limit (PEL) ceiling values and 5 times the ACGIH 8-hour TLVs (mean ratios $=1.09$ and 1.05, respectively). A poor correlation was observed between ERPG-2 and ACGIH TLV ceiling $($ TLV-C) values (mean ratio $=0.2$ ). Data were not presented for the ratio of ERPG-2 to TLV values. However, on the basis of the good correlation with 5 times the 8-h TLVs, the correlation between ERPG-2 and unadjusted 8-h TLVs is probably very poor.

The study by Craig et al. (1995) confirms the stratification observed when unadjusted ACGIH TLV-C values, unadjusted 8-h TLV values, or 3 times the 8-h TLV values are used as surrogates for ERPG-2 values. Also, on the basis of the good correlation between the EPA LOC and ERPG-2 values, and since many EPA LOC values were derived by dividing the IDLH value by 10 and many IDLH values were derived by dividing an $\mathrm{LC}_{50}$ value by 10 , a relationship between the ERPG-2 and $\mathrm{LC}_{50} / 100$ is inferred.

Woudenberg and Van Der Torn (1992) performed a number of nonparametric and parametric comparisons of various exposure limits, including ERPGs (3, 2, and 1), IDLH values, ACGIH short-term exposure limits (STELs), 50\% decrease in mean respiratory rate $\left(\mathrm{RD}_{50}\right)$ values, Health and Safety Executive (HSE) specified levels of toxicity (SLOTs), and National Research Council EEGLs. Woudenberg and Van Der Torn also calculated ratios of ERPG-3 to ERPG-2. At the time these analyses were performed, ERPG values were available for only 25 chemicals.

For ERPG-2, the nonparameteric comparison procedure identified a cluster with only EEGLs. This trend was confirmed by using parametric procedures. The ratio of mean EEGL to mean ERPG-2 was 1, with a range of 0.1 to $3.3(\mathrm{~N}=7)$. For ERPG-3, the nonparametric procedure identified a cluster with IDLH values. When the limits were scaled to a common time period, the $\mathrm{RD}_{50}$ value was included in this cluster. The mean ERPG-3/IDLH ratio was 0.92, with a range of 0.125 to $5(\mathrm{~N}=16)$. The mean $\mathrm{RD}_{50} / \mathrm{ERPG}-3$ ratio was 2 , with a range of 0.1 to $7.8(\mathrm{~N}=12)$. In the above ratios, the values are not scaled to a common time period. The ratio ERPG-2/ERPG-3 was 0.2 , with a range of 0.01 to 0.4 , indicating a fivefold difference between ERPG-2 and ERPG-3.

Two additional analyses were performed as part of ERG1996 study to further improve the values used in the ERG. Data from 65 ERPGs were used in the analyses $(\mathrm{N}=65)$. The relationship between the ratio of the key $\mathrm{LC}_{50}$ value and the ERPG-3 was analyzed. The key $\mathrm{LC}_{50}$ values were selected from the ERPG documentation, except where noted. With the exception of one chemical, dimethylamine, the $\mathrm{LC}_{50}$ values were for 30 -min to 4-h exposure durations. Non-1-h values were adjusted to $1 \mathrm{~h}$. In this study, the ranking order described in Section 4.2 was used. The ratio of mean LC 50 to mean ERPG-3 was 25.7, with a median of 17 and range of 3 to 168 . The ratio of ERPG-3 to ERPG-2 was also analyzed. The ratio of mean ERPG-3 to mean ERPG-2 was 6.6, with a median of 5 and range of 1-50. 
For both ratios, the median values are slightly lower than the mean values, indicating that a few high values are influencing the means. For such data, the median values, which minimize the influence of extreme values, are the best estimate of central tendency. Considering the median value, the $1-\mathrm{h} \mathrm{LC}_{50} / 20$ value (i.e., $0.05 \times \mathrm{LC}_{50}$ ) is a reasonable approximation for the ERPG-3 value. Since ERPG-3 values are based on protection from life-threatening health effects, the relationship between $\mathrm{LC}_{50}$ and ERPG-3 has biological relevance. The product of the ratio of $\mathrm{LC}_{50} / \mathrm{ERPG}-3$ and ERPG-3/ERPG-2 is calculated as $17 \times 5=85$. Therefore, $1-\mathrm{h} \mathrm{LC}_{50} / 100$ values appear to be a reasonable and slightly conservative approximation for ERPG-2 values. Note that this relationship is empirical, since ERPG-2 values are based on a variety of serious health effects rather than lethality.

\subsubsection{Expert Panel Review}

On May 4, 1995, an independent panel of expert toxicologists convened to make recommendations on the use of health criteria for the 1996 version of the ERG (Dunn et al. 1996). The panel made a number of recommendations for improving the consistency of criteria and for reducing stratification. They are presented below.

- Avoid using occupational health guidelines based on cancer since their use results in significant stratification.

- Avoid using occupational health guidelines based on effects other than irritation since their use introduces a similar bias.

- Using acute inhalation toxicity data (e.g., LC50 values) in place of occupational health guidelines probably improves the consistency of the health criteria. However, the inconsistent use of these data has a high potential for introducing bias. To increase consistency, a scheme that considers species, exposure duration differences, and data sources should be developed and uniformly applied to data on individual chemicals.

In addition, the panel recommended identifying new potential sources of information for developing the health criteria. For example, additional acute toxicity data may be found in the documentation of the AIHA ERPGs. These documents cite proprietary data not included in the open literature. Also, the panel recommended making more use of ERPG values by using existing ERPGs for closely related analogs that have limited toxicity data and no ERPGs.

The recommendations from the expert panel, and information gained from the studies and analyses described above, were incorporated into an updated procedure for developing the health criteria used to calculate Initial Isolation and PADs. 


\subsection{PROCEDURE USED TO DEVELOP HEALTH CRITERIA}

Modeling considerations indicated a need for three values for each chemical. These values were a 1-h life-threatening value for determining the Initial Isolation Distances, and 1-h and 15-min protective action values for determining PADs. The procedure used to derive these values is described below.

\subsubsection{Use of ERPGs}

When published or approved ERPGs were available for the chemical of interest, ERPG-2 was used as the 1-h protective action criteria. ERPG- $2 \times 2$ was used to estimate 15-min protective criteria. Use of the twofold factor is explained in Section 4.2.2 below. If ERPGs were available for a closely related structural analog, the ERPG-2 value for the structural analog was used for the chemical of interest, as described above.

\subsubsection{Use of Acute Inhalation Lethality Data in Animals}

When ERPGs were not available, health criteria were derived by using $\mathrm{LC}_{50}$ data and lowest reported lethal concentration $\left(\mathrm{LC}_{\mathrm{LO}}\right)$ data from acute inhalation studies on animals. When such data were not available for a chemical of interest, we used corresponding data for a structural analog. For example, for certain isocyanates for which there were no acute lethality data, data for methyl or butyl isocyanate could be used. Similarly, data for boron trichloride could be used for boron tribromide.

Several factors were considered in selecting and using the $\mathrm{LC}_{50}$ and $\mathrm{LC}_{\mathrm{LO}}$ data. These factors included species, time, data source, and structure activity considerations. These factors are discussed below and the resulting ranking of studies appears in Table 4.1.

\subsubsection{Species Considerations}

Data from studies using rats and mice were preferred for several reasons. Studies conducted with these species tend to use standardized protocols. Also, there is a wealth of comparative lethality data on rats and mice and much less comparative data on other species. Data on primates are rare, so using these data would limit the ability to compare responses across chemicals. However, acute lethal responses in this species might more closely simulate human responses. Therefore, when such data were available, they were generally included after the data for rats and mice but before data for other species, as presented in Table 4.1. The amount of comparative data on rabbits is limited. Results on this species are not as representative as data on primates, and so they appear lower in the ranking scheme. 
TABLE 4.1 Order of Acute Lethality Data for Estimating Health Criteria

\begin{tabular}{|c|c|c|c|}
\hline Rank & Data & Rank & Data \\
\hline 1 & $1-\mathrm{h} \mathrm{CC}_{50}$ in rats & 14 & 2- to $4-\mathrm{h} \mathrm{LC}_{50}$ in dogs \\
\hline 2 & 2- to $4-\mathrm{LC}_{50}$ in rats & 15 & $30 \min \mathrm{LC}_{50}$ in dogs \\
\hline 3 & $30-\mathrm{min} \mathrm{LC}_{50}$ in rats & 16 & 6- to 8-h $\mathrm{LC}_{50}$ in dogs \\
\hline 4 & $1-\mathrm{h} \mathrm{LC}_{50}$ in mice & 17 & $1-\mathrm{h} \mathrm{LC}_{50}$ in guinea pigs \\
\hline 5 & 2- to $4-\mathrm{LCC}_{50}$ in mice & 18 & 2- to $4-\mathrm{h} \mathrm{LC}_{50}$ in guinea pigs \\
\hline 6 & $30-\min \mathrm{LC}_{50}$ in mice & 19 & $30 \mathrm{~min} \mathrm{LC}_{50}$ in guinea pigs \\
\hline 7 & 6- to 8-h $\mathrm{LC}_{50}$ in rats & 20 & 6 - to 8 -h $\mathrm{LC}_{50}$ in guinea pigs \\
\hline 8 & 6- to 8 - $\mathrm{h}_{50}$ in mice & 21 & $1-\mathrm{h} \mathrm{LC}_{50}$ in rabbits \\
\hline 9 & $1-\mathrm{h} \mathrm{LC}_{50}$ in primates & 22 & 2- to 4-h $\mathrm{LC}_{50}$ rabbits \\
\hline 10 & 2- to $4-\mathrm{LC}_{50}$ in primates & 23 & $30-\min \mathrm{LC}_{50}$ in rabbits \\
\hline 11 & $30-\mathrm{min} \mathrm{LC}_{50}$ in primates & 24 & 6- to 8 -h $\mathrm{LC}_{50}$ in rabbits \\
\hline 12 & 6 - to 8 - $\mathrm{h} \mathrm{LC}_{50}$ in primates & 25 & 1-h $\mathrm{LC}_{\mathrm{LO}}$ in rats ${ }^{\mathrm{a}}$ \\
\hline 13 & $1-\mathrm{h} \mathrm{LC}_{50}$ in dogs & 26 & 2- to 4-h $\mathrm{LC}_{\mathrm{LO}}$ in rats, etc. ${ }^{\mathrm{a}}$ \\
\hline
\end{tabular}

a Rank 25 through 50 for $\mathrm{LC}_{\mathrm{LO}}$ data follow the same order as Rank 1 through 24 for $\mathrm{LC}_{50}$ data shown.

\subsubsection{Exposure Duration Considerations}

Data from 1-h exposures were preferred, since data from this duration require no adjustments. The most commonly reported acute lethality studies are for 1-h and 4-h exposure durations. Therefore, use of data from studies of this duration provides a measure of consistency in estimating health criteria. There is also a tendency for 1-h and 4-h $\mathrm{LC}_{50}$ values to have been calculated by using standard protocols. For exposures less than $30 \mathrm{~min}$ long, concerns over chamber equilibration time (T99) increased. For exposures more than $4 \mathrm{~h}$ long, concerns that effects other than acute lethal effects might influence the study results increased. Limited confidence was placed on data reported as $\mathrm{LC}_{\mathrm{LO}}$. In these studies, no information was available concerning the slope of the dose response curve. Also, in some studies that used $\mathrm{LC}_{\mathrm{LO}}$ values, $100 \%$ mortality was observed.

Data from non-1-h exposures were adjusted to predict results for 1-h exposures. To develop an approach for making the adjustments, various reports published by investigators who have examined the relationship between exposure duration and acute mortality response were reviewed (Doe and Milburn 1983; Haber 1924; Klimisch et al. 1987; Ten Berge et al. 1986).

In the simplest case, where the inhaled substance accumulates in the body and is not rapidly destroyed or excreted, the dose accumulated is directly proportional to the concentration $c$ and the exposure time $t$, and uptake is linear. This concept, known as Haber's rule or law, would result in the following relationship:

$$
W=c t
$$


where $W$ is a constant dose specific for any given effect. This relationship is applicable for many reactive gases or highly lipid-soluble vapors over a limited range of concentrations and time.

However, many other relationships are possible. For example, for chemicals that are excreted as fast as they are inhaled and for which accumulation does not occur until a certain threshold concentration is reached, the following generalized dose-response equation applies:

$$
W=(c-a) t^{b}
$$

where $a$ is the threshold concentration and $b$ is derived from experimental data.

For a significant percentage of chemicals, the following relationship has been observed:

$$
L C_{50}\left(T_{1}\right)=L C_{50}\left(T_{o}\right)\left(\frac{T_{1}}{T_{o}}\right)^{1 / n},
$$

where

$$
\begin{aligned}
& T_{1}=\text { actual exposure time } \\
& T_{\mathrm{O}}=\text { experimental exposure time, and } \\
& n=\text { constant. }
\end{aligned}
$$

Klimisch et al. (1987), citing Doe and Milburn (1983), found that for many chemicals, $n$ centers around a value of 0.5. Ten Berge et al. (1986) determined that for 18 of 20 chemicals studied, $n$ values were greater than 0.3 .

For deriving the health criteria, acute lethality data for exposure durations $T_{O}$ longer than $1 \mathrm{~h}$ were normalized by using the following dose-response function:

$$
L C_{50}(1 h r)=L C_{50}\left(T_{o}\right)\left(\frac{1 h r}{T_{o}}\right)^{0.5}
$$

This approach predicts lower $\mathrm{LC}_{50} / \mathrm{LC}_{\mathrm{LO}}$ values than does Haber's rule. Acute lethality data for exposure durations $T_{O}$ of less than $1 \mathrm{~h}$ were predicted by using a linear dose-response function (i.e., Haber's rule):

$$
L C_{50}(1 h r)=L C_{50}\left(T_{o}\right)\left(\frac{1 h r}{T_{o}}\right)
$$




\subsubsection{Data Source Considerations}

The source of the data is critical. Information from main-stream, peer-reviewed toxicology and industrial hygiene journals is preferable to information from ancillary, non-peerreviewed sources. The publication date may also be important. Many studies conducted before 1950 did not include analytical verification of concentrations. Information from foreign journals tends to contain more transposition errors, especially as cited in the NIOSH Registry of Toxic Effects of Chemical Substances.

\subsubsection{Adjustment Factors}

As described in Section 4.1, the $1-\mathrm{h} \mathrm{LC}_{50}$ or adjusted $1-\mathrm{h} \mathrm{LC}_{50} / 100$ is a reasonable estimate of ERPG-2. Also, a fivefold factor is a reasonable central tendency adjustment factor between ERPG-2 and ERPG-3. Therefore, 1-h or adjusted 1-h $\mathrm{LC}_{50}$ or $\mathrm{LC}_{\mathrm{LO}}$ values were divided by 100 to estimate $1-\mathrm{h}$ protective health criteria, and this value was multiplied by 5 to estimate the 1-h life-threatening health criteria.

Members of the AIHA ERPG Committee indicate that when 1-h ERPGs are extrapolated

to values of shorter duration, there is concern that the potential effects of peak, high-level exposures should be minimized. A default value of 2 was suggested for these purposes. Therefore, to estimate 15-min protective health criteria from 1-h values, a factor of 2 was employed in estimating the 15-min criteria from the 1-h criteria.

\subsubsection{Use of Alternative Health Criteria}

For a few select chemicals, National Research Council EEGLs, Emergency Exposure Levels (EELs) published in the AIHA Journal were used to develop health criteria. For one chemical, data on oral $\mathrm{LD}_{50}$ in animals were used to estimate an $\mathrm{LC}_{50}$ value and to derive health criteria. Standard assumptions of animal inhalation rate and body weight were used.

\subsubsection{Summary}

By building on past efforts, an updated procedure was developed to provide health criteria for use in determining Initial Isolation and Protective Action Distances. The new procedure incorporated additional ERPG values published since 1992 and expanded the use of existing ERPGs by applying them to structural analogs that otherwise had limited or no available acute toxicity data. A strategy was developed to reduce the stratification of PADs according to health criteria. The strategy involved minimizing the use of occupational health criteria through increased use of acute lethality data and more consistent selection and use of acute lethality data.

A summary of the basis for health criteria of the 166 primary chemicals in the ERG analysis appears in Table 4.2. Documentation of the health criteria for individual chemicals is 
TABLE 4.2 Summary of the Basis for Health Criteria Used to Prepare the ERG2004

\begin{tabular}{|c|c|c|}
\hline Basis of Health Criteria & $\begin{array}{c}\text { No. of } \\
\text { Materials }\end{array}$ & Percentage \\
\hline ERPG for chemical of concern & 44 & 27 \\
\hline ERPG for structurally similar chemical & 20 & 12 \\
\hline Subtotal for ERPGs & 64 & 39 \\
\hline $\mathrm{LC}_{50}$ for chemical of concern & 80 & 49 \\
\hline $\mathrm{LC}_{50}$ for structurally similar chemical & 10 & 6 \\
\hline Subtotal for $\mathrm{LC}_{50} \mathrm{~s}$ & 90 & 54 \\
\hline $\mathrm{LC}_{\mathrm{LO}}$ for chemical of concern & 9 & 5 \\
\hline AIHA EEL & 2 & 1 \\
\hline Oral toxicity data & 1 & 1 \\
\hline Total & 166 & 100 \\
\hline
\end{tabular}

presented in Appendix B. For 64 chemicals (39\% of them), ERPGs or ERPGs for a structurally similar chemical formed the basis of the health criteria. For 90 chemicals $(54 \%), \mathrm{LC}_{50}$ values or $\mathrm{LC}_{50}$ values for a structurally similar chemical were used to develop the health criteria. For 9 chemicals $(5 \%), L_{L O}$ values for the chemical or a structurally similar chemical formed the basis of the health criteria. National Research Council EELs were employed for 2 chemicals, and for 1 chemical, oral toxicity data were used to estimate an inhalation $\mathrm{LC}_{50}$ and to derive the health criteria.

\subsection{COMPARISON TO OTHER PROCEDURES}

A number of other procedures have been developed for deriving health criteria used in evaluating accidental releases of chemicals. These include procedures developed by the EPA for deriving LOCs to evaluate releases of extremely hazardous substances (EPA 1991) and other procedures developed by the EPA for performing consequence analyses to comply with requirements of Section $112 \mathrm{R}$ of the Clean Air Act (EPA 1996).

In the EPA LOC procedure, the NIOSH/OSHA IDLH/10 is the preferred value for deriving the LOC. Since IDLH values were developed during the Standards Completion Program for the purpose of selecting respirators in the event of an emergency in the workplace, the tenfold uncertainty factor is intended to account for the greater sensitivity of the general population versus the worker population. When IDLH values are unavailable, estimated IDLH values based on $\mathrm{LC}_{50} / 100, \mathrm{LC}_{\mathrm{LO}}, \mathrm{LD}_{50} / 100$, or $\mathrm{LD}_{\mathrm{LO}} / 100$ are used. As a third choice, ACGIH TLVs (8-h time-weighted average TLVs [TLV-TWA], STEL, and TLV-C values) and National Research Council EEGLs are also used to derive a number of LOCs. AIHA ERPGs were cited as alternative criteria to use to develop LOCs. However, because only 15 draft ERPGs were 
available at the time the LOC guidance was developed, ERPGs did not form the basis for any LOC.

In the procedure described by NIOSH to develop IDLH values, human data are preferred. However, since reliable human data are rarely available, many of the IDLH values are based on adjusting the results of acute inhalation lethality data in animals to a 30-min exposure duration by using the calculation $\mathrm{LC}_{50}(30 \mathrm{~min})=\mathrm{LC}_{50}(\mathrm{~T}) \times(\mathrm{T} / 0.5)^{1 / 3}$, then dividing by 10 . Therefore, many LOCs are based on adjusted $\mathrm{LC}_{50}$ or $\mathrm{LC}_{\mathrm{LO}}$ data divided by 100.

Several similarities exist between the procedure used by the EPA to develop LOCs and the procedure used by DOT to develop health criteria for deriving Initial Isolation and Protection Action Distances. Many LOC and DOT 1 -h protective levels are based on adjusted $\mathrm{LC}_{50}$ or $\mathrm{LC}_{\mathrm{LO}}$ values divided by 100 . Also, various alternative values such as National Research Council EEGLs and ACGIH TLVs are considered as "last resort" criteria.

There are also several important differences in these two procedures. In the DOT approach, AIHA ERPGs are the preferred choice for deriving health criteria. We believe this to be a sounder approach, since ERPG values are considered by many authorities to be the best available criteria for evaluating accidental releases. Also, in the DOT procedure, when it was necessary to use acute lethality data in animals, a critical review of the available data was performed, which included a scheme for selecting the best study when multiple studies were available. In the DOT approach, when $\mathrm{LC}_{\mathrm{LO}}$ data were employed, use of a hundredfold instead of a tenfold uncertainty factor was maintained. This recommendation was based on the concept that a significant number of $\mathrm{LC}_{\mathrm{LO}}$ values represent concentrations that produced a significant percentage of mortality, including $100 \%$ mortality.

In the approach described in the guidance document for off-site consequence analysis (EPA 1996), ERPGs are recommended as the preferred values, followed by LOC values. Since ERPGs are recommended as first priority in the DOT scheme, followed by the time-adjusted $\mathrm{LC}_{50} / 100$, and many LOCs are based on $\mathrm{LC}_{50} / 100$, the two approaches are quite similar. However, as described above, there are differences in the methods used to select the acute lethality data.

\subsection{FUTURE MODIFICATIONS}

\subsubsection{Additions of New ERPG Values}

Through continued efforts of the AIHA ERPG Committee, ERPGs for additional chemicals are being provided at a rate of 7-10 per year, and already published values are occasionally revised. Not all of the chemicals on the ERPG list appear in the Table of Initial Isolation and Protective Action Distances, since many of the ERPG chemicals do not meet the specific toxicity and physical criteria for listing in the Table. However, when new ERPGs are available for chemicals on the Table, the values will be incorporated into the development of Isolation and Protective Action Distances in future additions of the ERG. 


\subsubsection{Acute Exposure Guidance Levels}

A Federal Advisory Committee for Acute Exposure Guidance Levels (AEGLs) was approved in 1995. This committee is composed of scientists representing federal, state, and local agencies and organizations from the private sector with an interest in emergency planning, prevention, and response programs for acutely toxic chemicals. The purpose of the committee is to develop AEGLs that will meet the needs of various organizations (EPA, NIOSH, OSHA, DOT, U.S. Department of Defense, U.S. Department of Energy, Agency for Toxic Substances Disease Registry, and private industry). AEGLs represent threshold exposure limits for the general public and are applicable to emergency exposure periods ranging from $10 \mathrm{~min}$ to $8 \mathrm{~h}$. AEGL-1 and AEGL-2 levels, and AEGL-3 levels as appropriate, are developed for each of five exposure periods (10 and $30 \mathrm{~min}, 1 \mathrm{~h}, 4 \mathrm{~h}$, and $8 \mathrm{~h}$ ) and are distinguished by varying degrees of severity of toxic effects. It is believed that the recommended exposure levels are applicable to the general population, including infants, children, and other individuals who may be sensitive and susceptible. The definitions of AEGL-1, AEGL-2 and AEGL-3 are very close to those for the ERPG values:

- AEGL-1 is the airborne concentration of a substance above which it is predicted that the general population, including susceptible individuals, could experience notable discomfort, irritation, or certain asymptomatic, non-sensory effects. However, the effects are not disabling and are transient and reversible upon cessation of exposure.

- AEGL-2 is the airborne concentration of a substance above which it is predicted that the general population, including susceptible individuals, could experience irreversible or other serious, long-lasting adverse health effects, or an impaired ability to escape.

- AEGL-3 is the airborne concentration of a substance above which it is predicted that the general population, including susceptible individuals, could experience life-threatening health effects or death.

As these are extensively peer reviewed acute health criteria applicable to emergency response situations, they are directly applicable for use in the ERG analysis for chemicals that do not have ERPGs. A few final AEGL values and many draft values of AEGLs were available at the time of the ERG2004 analysis; however, the AEGLs available at the time repeated already published ERPG values, and since the values were substantially different in some cases, the decision was made to postpone inclusion of AEGLs. AEGLs are being finalized at a rate of 1020 chemicals a year, so by the time the analysis for the next ERG edition commences, many final AEGL values will be available. We anticipate that many of these will be for chemicals for which no ERPG exists, so it is likely that AEGLs will be incorporated in the ERG analysis framework in the future. However, there are a few questions that will need to be resolved for a seamless integration.

The main question is whether the AEGL-2 is exactly comparable to the ERPG-2. The ERG analysis was developed based on ERPG-2 levels. While the definitions of the ERPG-2 and 
AEGL-2 appear very similar, the final AEGL-2 values are often lower than their ERPG-2 counterparts. This could have a marked effect on PAD values. Table 4.3 shows 8 TIH materials for which both ERPG and final AEGL values were available in February 2005, and the percent change in the nighttime, large spill PAD if the AEGL is used in place of the ERPG. For 4 of the materials the AEGL values were substantially lower, for 3 they were slightly higher, and for 1 they were the same. The percent changes in the nighttime, large spill PAD ranges from a decrease of $16 \%$ to an increase of $106 \%$, with the average being an increase of $21 \%$. The reason for this increase may indeed be a slight difference in their definitions, since the AEGL-2 definition contains the statement "...general population, including susceptible individuals ...", whereas the ERPG-2 values are valid for "nearly all" individuals.

If this trend continues, incorporating large numbers of AEGLs could significantly raise the PAD values if the rest of the analysis is unchanged. This issue will be thoroughly examined as part of the next ERG analysis. A related question will be whether to use the AEGL or ERPG if both values exist for the same chemical.

\subsection{GLOSSARY FOR SECTION 4}

ACGIH 8-hour TLV: American Conference of Governmental Industrial Hygienists 8-h threshold limit value (ACGIH 1995). This is a time-weighted average concentration to which nearly all workers may be repeatedly exposed, day after day, without adverse effect.

TABLE 4.3 Comparison of ERPG-2 Values with Final AEGL-2 Values for 8 TIH Materials and the \% Change in Large Spill Nighttime Distance if the AEGL-2 Value Replaced the ERPG-2 Value in the ERG Analysis

\begin{tabular}{|c|c|c|c|c|c|}
\hline Chemical & 1-h ERPG-2 & $\begin{array}{c}\text { 15-min } \\
\text { ERPG-2 }\end{array}$ & $\begin{array}{c}1-\mathrm{h} \\
\text { AEGL-2 }\end{array}$ & $\begin{array}{c}\text { 15-min } \\
\text { AEGL-2 }^{\mathrm{a}}\end{array}$ & $\begin{array}{c}\% \text { Change in } \\
\text { Large Spill } \\
\text { Nighttime } \\
\text { Distance }\end{array}$ \\
\hline Hydrogen cyanide & 10 & 20 & 7.1 & 14.2 & +21 \\
\hline Phosgene & 0.2 & 0.4 & 0.3 & 0.6 & -16 \\
\hline Methyl isocyanate & 0.5 & 1.0 & 0.067 & 0.26 & +106 \\
\hline Hydrogen chloride & 20 & 40 & 22 & 86 & -7.3 \\
\hline Hydrogen fluoride & 20 & 40 & 24 & 48 & -10 \\
\hline Chlorine & 3 & 6 & 2 & 2.8 & +29 \\
\hline Arsine & 0.5 & 1 & 0.17 & 0.26 & +47 \\
\hline Diborane & 1 & 2 & 1 & 2 & 0 \\
\hline Average change & & & & & +21 \\
\hline
\end{tabular}

a 15-min AEGL-2 values were estimated using the published 10- and 30-min values. 
ACGIH TLV STEL: ACGIH short-term exposure limit (ACGIH 1995). The concentration to which workers can be exposed continuously for a short period of time without suffering from (1) irritation, (2) chronic or irreversible tissue damage, or (3) narcosis of sufficient degree to increase the likelihood of accidental injury, impair self-rescue, or materially reduce work efficiency, provided that the daily TLV-TWA is not exceeded.

ACGIH TLV Ceiling: ACGIH threshold limit value ceiling (ACGIH 1995). This is the concentration that should not be exceeded during any part of the workday.

AEGL: Acute Exposure Guidance Levels (AEGLs) — described in detail in Section 4.4.2.

AIHA EEL: Emergency Exposure Level published in the AIHA Journal (Frawley et al. 1964). This is the concentration of contaminant that can be tolerated without adversely affecting health but not necessarily without acute discomfort or other evidence of irritation or intoxication. The level is intended to provide guidance in managing single, brief exposures to airborne contaminants in the working environment.

AIHA ERPGs: AIHA Emergency Response Planning Guidelines (AIHA 2004) — described in detail in Section 4.1.1.

EPA LOC: U.S. Environmental Protection Agency level of concern (EPA 1991). The concentration of an extremely hazardous substance in the air above which there may be serious irreversible health effects or death as a result of a single exposure for a relatively short period of time.

HSE SLOT: Health and Safety Executive (England) specified level of toxicity (Turner and Fairhurst 1989).

LC50: median (50\%) lethal concentration; concentration that is lethal to $50 \%$ of the exposed population.

$\mathbf{L C}_{\mathbf{L O}}$ : lowest reported lethal concentration, i.e., that will produce a fatality in an exposed population.

NIOSH IDLH: NIOSH immediately dangerous to life and health level (NIOSH 1994). The maximum concentration from which, in the event of respirator failure, one could escape within $30 \mathrm{~min}$ without experiencing any escape-impairing (e.g., severe eye irritation) or irreversible health effects.

NRC EEGL: National Research Council Emergency Exposure Guidance Level (NRC 19841987). This level is the concentration of a substance in air (as gas, vapor, or aerosol) that will permit continued performance of specific tasks during rare emergency conditions lasting for periods of $1-24 \mathrm{~h}$.

OSHA PEL Ceiling: OSHA permissible exposure limit ceiling (OSHA 1989). This is the concentration that should not be exceeded during any part of the workday. 
$\mathbf{R D}_{50}$ : concentration producing a $50 \%$ respiratory rate decrease in test animals; used to estimate severe respiratory irritation. 


\section{SUMMARY}

Sections $2-4$ of this report detail the statistical methodology for developing safe distance distributions, the consequence models used in the analysis, and the health criteria that define the safe distance. In this section, we summarize the results, discuss the appearance of the numbers in the Table (which is contained in Appendix A), and then conclude with some potential extensions of this analysis for situations where more information is immediately available besides gross spill size and whether it is day or night.

\subsection{SAFE DISTANCE DISTRIBUTIONS AND PROTECTIVE ACTION DISTANCES}

The statistical accident scenario and consequence analysis set forth in previous sections resulted in a set of up to 1,000,000 hypothetical incidents for each material appearing in the Table. This set of incidents accounts for variability in container type, incident type, accident severity (i.e., release amount), location, time of day, time of year, and meteorology. Each of these scenarios was evaluated using detailed emission rate and atmospheric dispersion models to calculate downwind chemical concentration footprints with the safe distance for each incident defined as the distance downwind from the source at which the chemical concentration falls below the health protection criteria. The safe distance estimates for the entire set of hypothetical incidents considered in the analysis provide a safe-distance distribution corresponding to a wide spectrum of potential transportation-related releases. In the final step of the analysis, these incidents are categorized according to whether they occur during the day or at night and whether they involve small $(\leq 60$ gal) or large ( $>60$ gal) spills.

Examples of safe distance distributions for two chemicals are given in Figures 5.1-5.8. The first four figures show safe distance distributions for chlorine, a Hazard Zone B gas. Distributions are separated according to spill size (large or small) and time of day (day or night). Figures 5.5-5.8 provide corresponding results for a water-reactive material, trichlorosilane, which emits $\mathrm{HCl}$ when spilled into water. In all eight figures, the $50^{\text {th }}, 70^{\text {th }}, 80^{\text {th }}, 90^{\text {th }}$, and $95^{\text {th }}$ percentiles are identified. The PADs in the guidebook correspond with the $90^{\text {th }}$-percentile values for the individual categories. Tables 5.1 and 5.2 provide safe distance estimates at several percentiles for the chlorine and trichlorosilane distributions shown in the figures. Tables 5.3 and 5.4 provide corresponding data for two additional chemicals, phosphorous chloride (a Hazard Zone B liquid) and ethylene oxide (a Hazard Zone D gas). Results for phosphorous chloride in Table 5.3 are for land-based releases only. A separate set of safe distance distributions was developed for spills of phosphorous chloride into water.

As demonstrated in these examples, the safe distance distributions exhibit substantial tails, denoting the presence of low-probability/high-consequence events. A comparison of the $50^{\text {th }}$ - and $90^{\text {th }}$-percentile values reveals that the $90^{\text {th }}$-percentile values are often a factor of 4 above the $50^{\text {th }}$-percentile values. Clearly, use of the $90^{\text {th }}$-percentile value for the PAD affords a substantial level of protection for most incidents. The $95^{\text {th }}$ and $99^{\text {th }}$ percentiles do show that the PADs will not be sufficient for all incidents, however. The $99^{\text {th }}$-percentile events, corresponding 


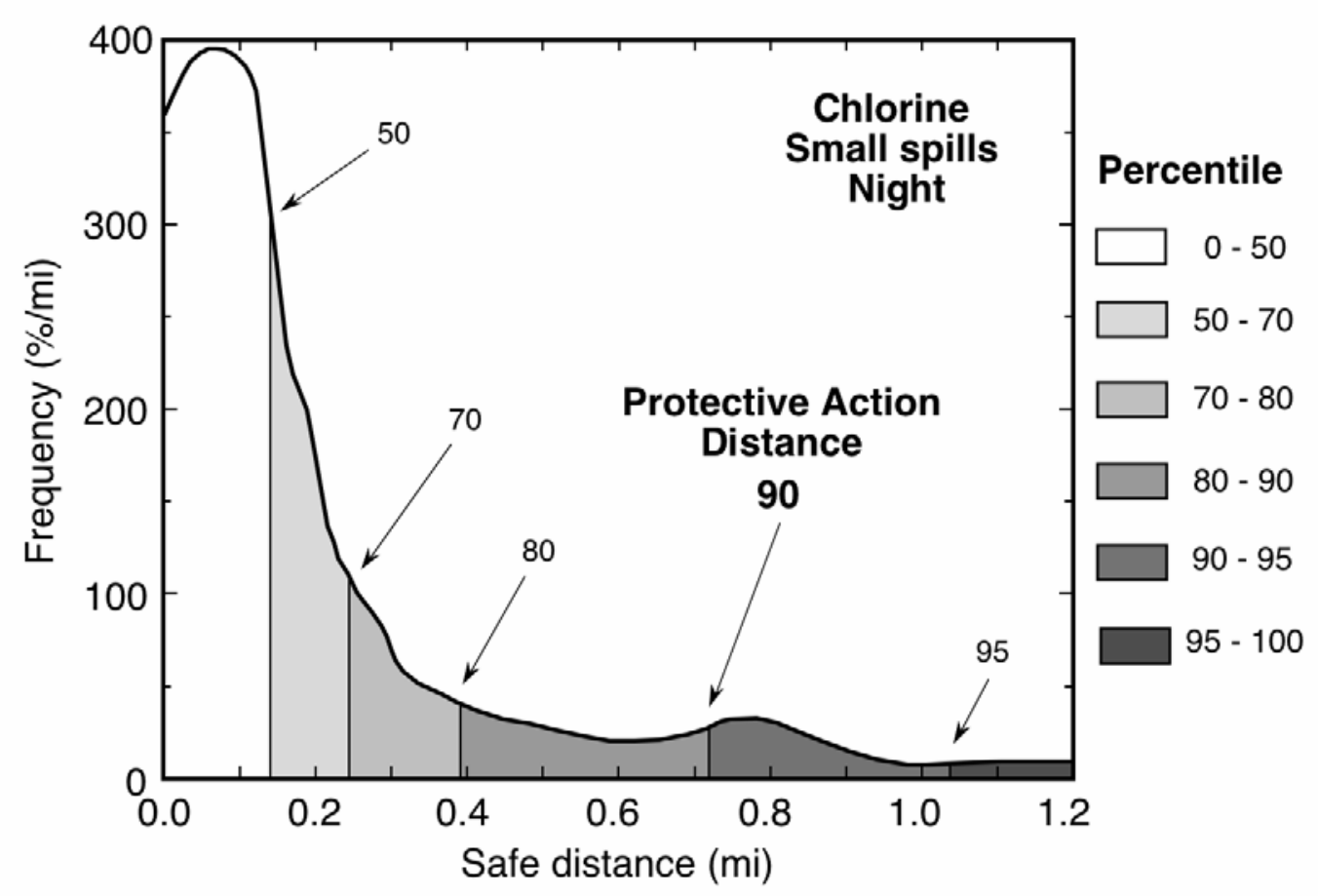

FIGURE 5.1 Frequency of Safe Distances for Small Nighttime Chlorine Spills as Determined in the ERG2004 Analysis

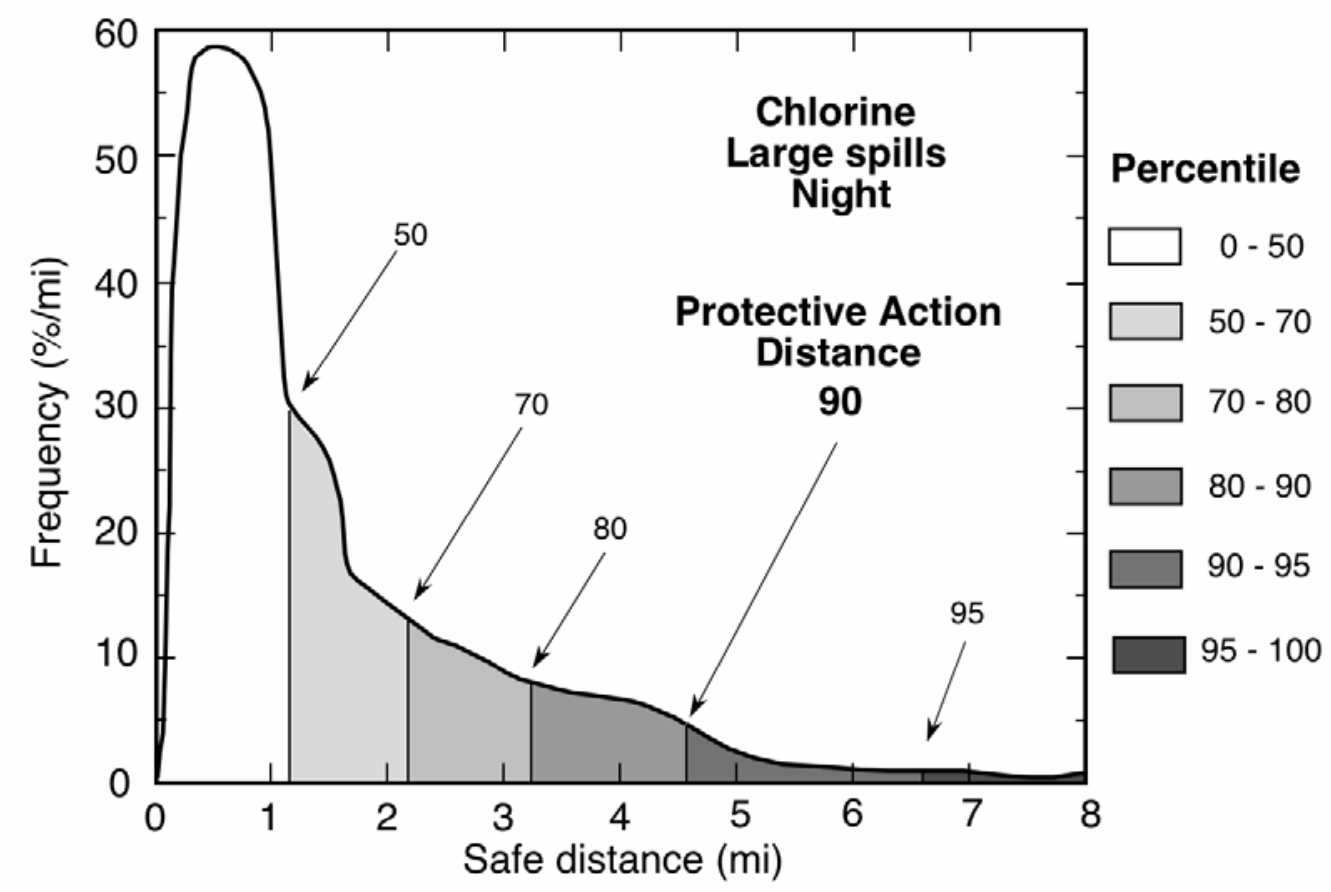

FIGURE 5.2 Frequency of Safe Distances for Large Nighttime Chlorine Spills as Determined in the ERG2004 Analysis 


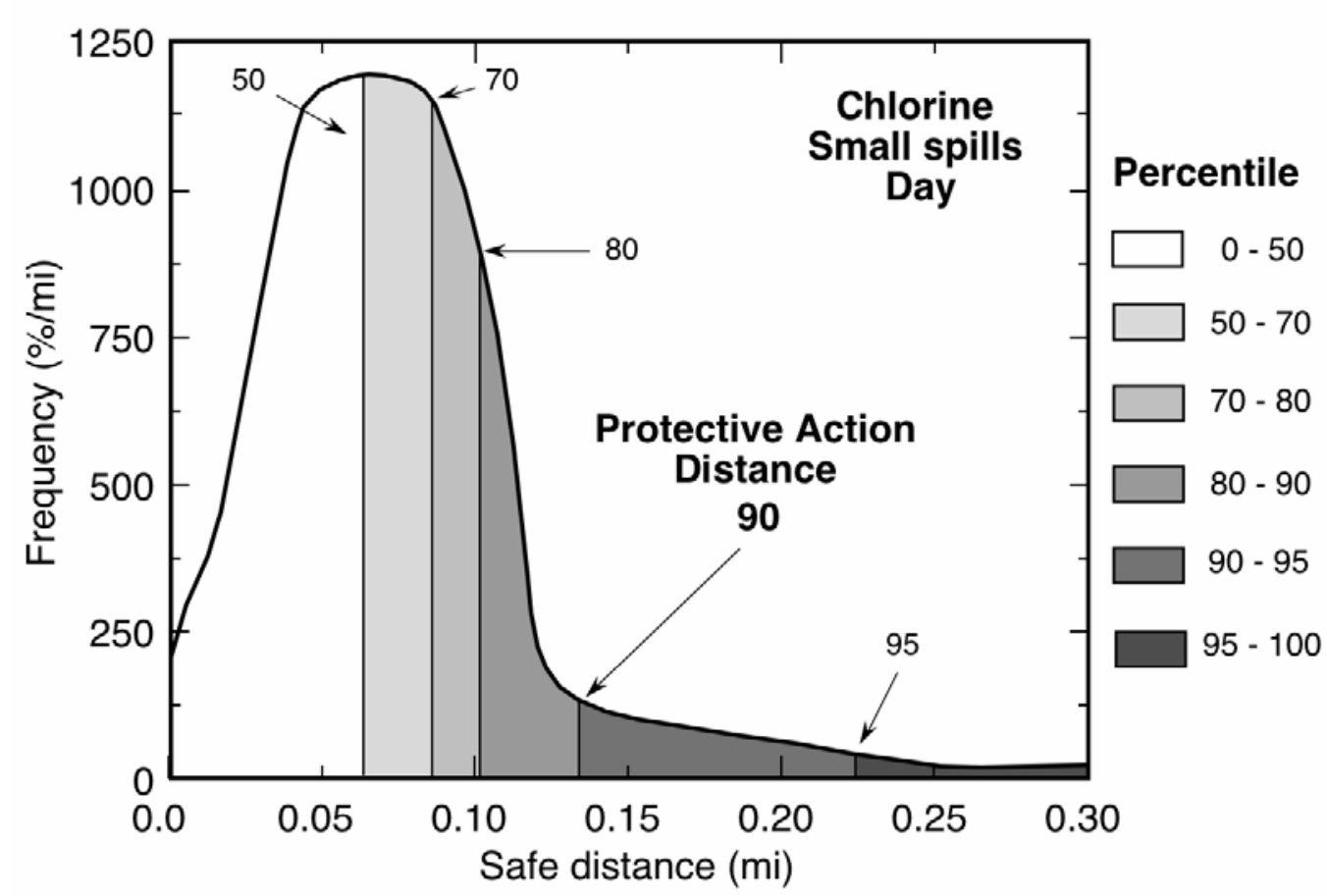

FIGURE 5.3 Frequency of Safe Distances for Small Daytime Chlorine Spills as Determined in the ERG2004 Analysis

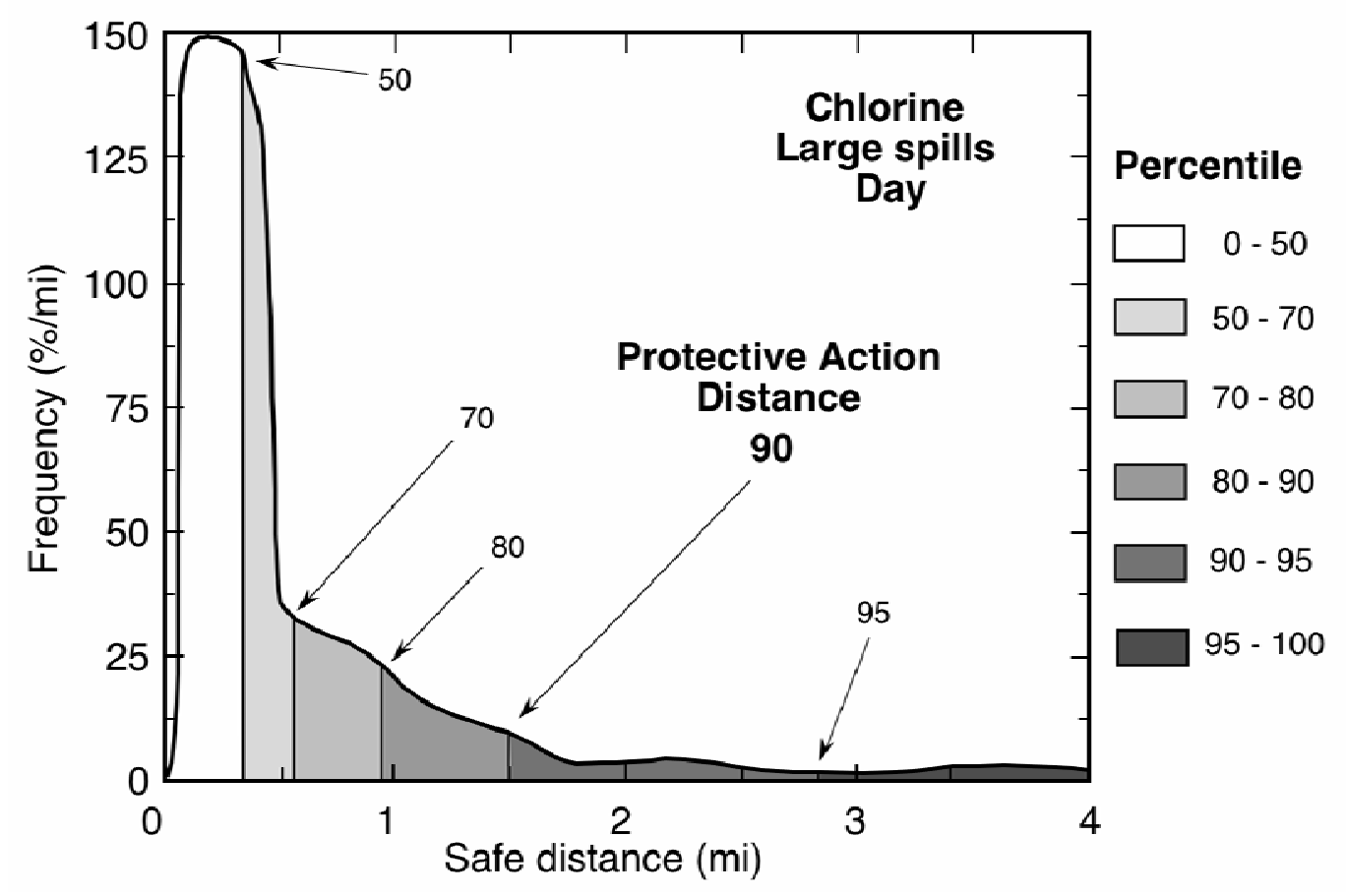

FIGURE 5.4 Frequency of Safe Distances for Large Daytime Chlorine Spills as Determined in the ERG2004 Analysis 


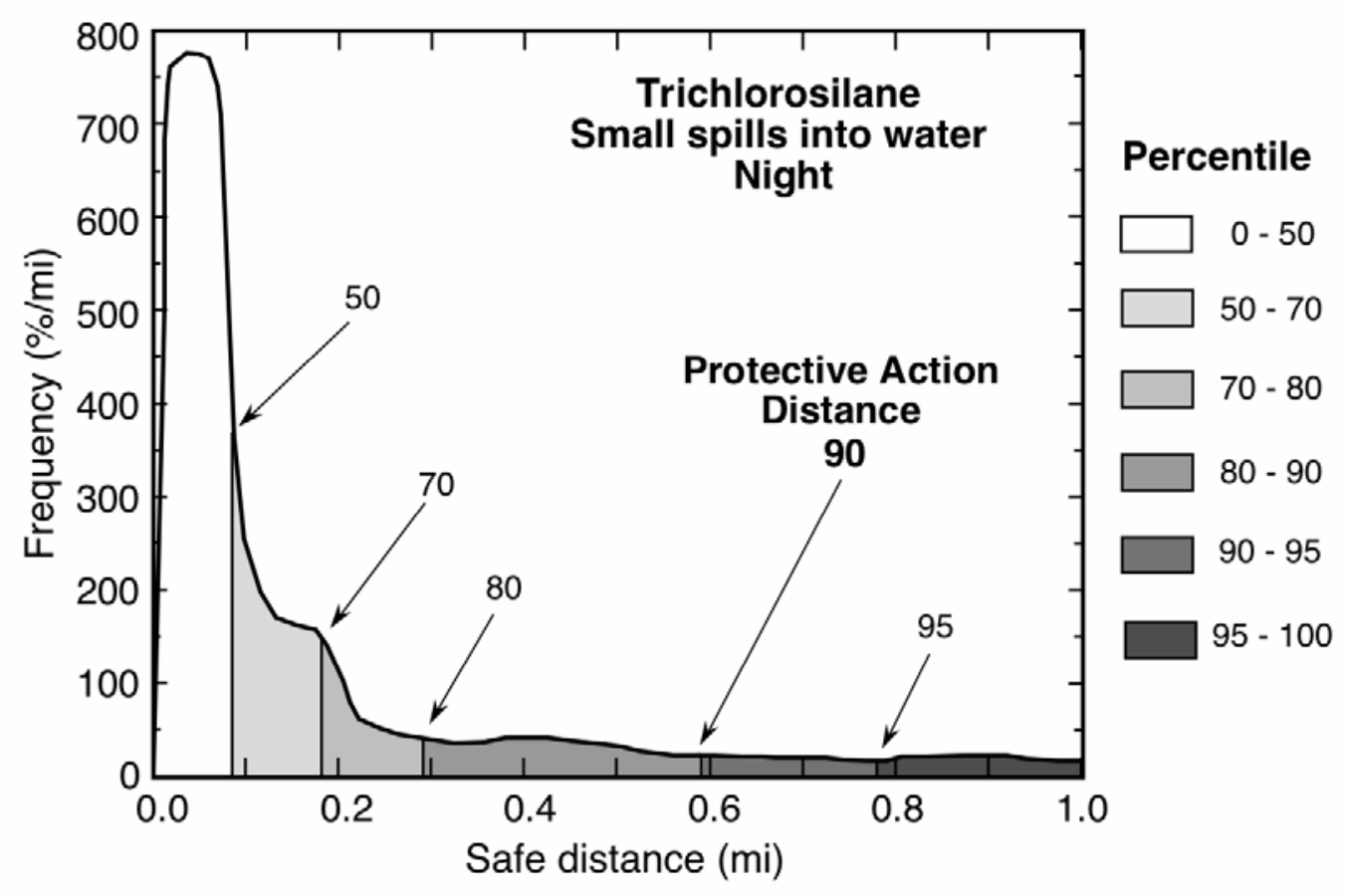

FIGURE 5.5 Frequency of Safe Distances for Small Nighttime Trichlorosilane Spills into Water as Determined in the ERG2004 Analysis

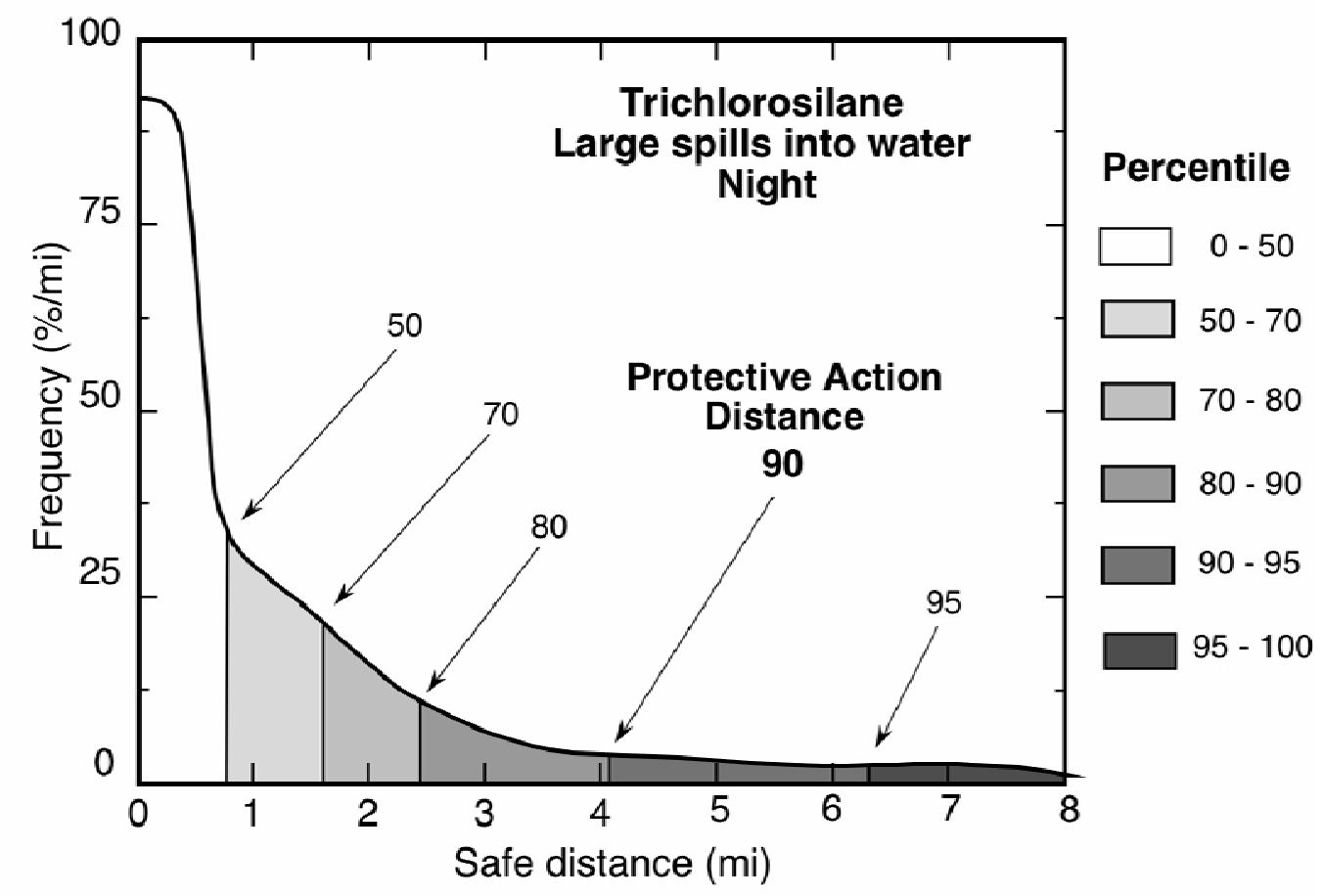

FIGURE 5.6 Frequency of Safe Distances for Large Nighttime

Trichlorosilane Spills into Water as Determined in the ERG2004 Analysis 


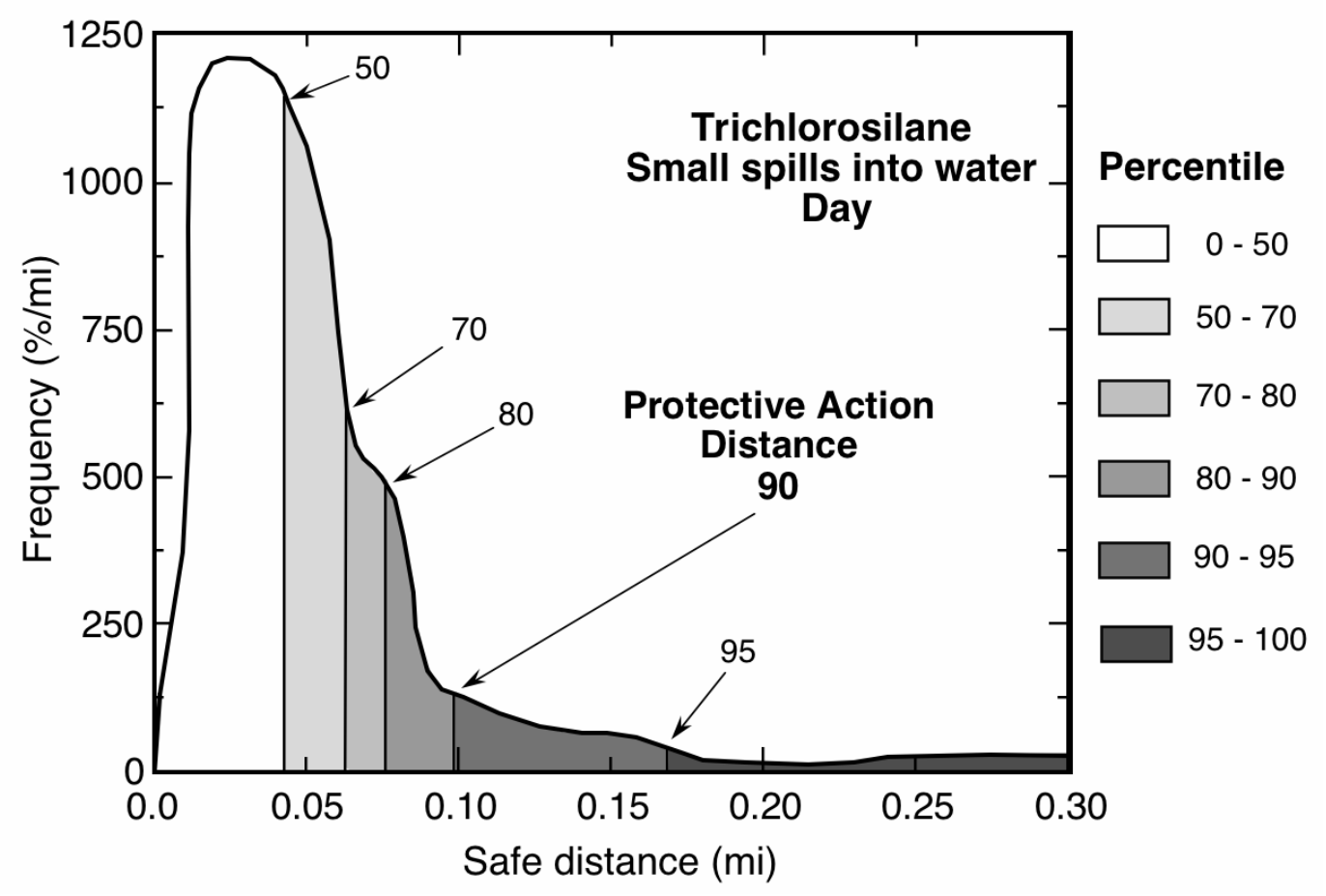

FIGURE 5.7 Frequency of Safe Distances for Small Daytime Trichlorosilane Spills into Water as Determined in the ERG2004 Analysis

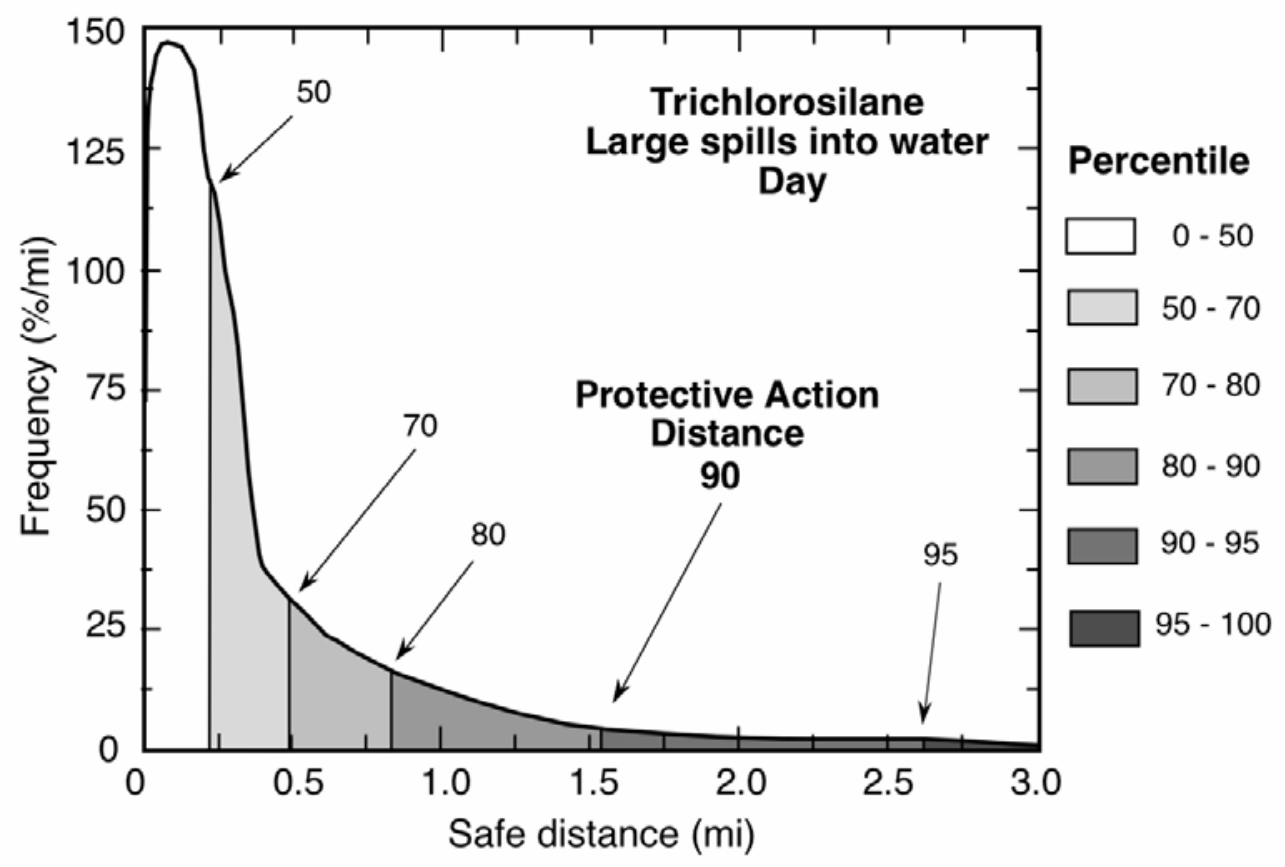

FIGURE 5.8 Frequency of Safe Distances for Large Daytime Trichlorosilane Spills into Water as Determined in the ERG2004 Analysis 
TABLE 5.1 Safe Distances at Several Percentiles for Chlorine (UN 1017) Releases as Determined in the ERG2004 Analysis

\begin{tabular}{lccccccc}
\hline & & \multicolumn{8}{c}{ Safe Distance (mi) for Given Percentile } \\
\cline { 3 - 8 } Release Size & Time & 50 & 70 & 80 & 90 & 95 & 99 \\
\hline \multirow{3}{*}{ Small } & & & & & & & \\
& Day & 0.06 & 0.08 & 0.10 & $\mathbf{0 . 1 3}$ & 0.22 & 0.72 \\
& Night & 0.13 & 0.23 & 0.39 & $\mathbf{0 . 7 2}$ & 1.03 & 1.71 \\
Large & & & & & & & \\
& Day & 0.32 & 0.53 & 0.95 & $\mathbf{1 . 4 9}$ & 2.83 & 8.22 \\
& Night & 1.03 & 2.13 & 3.12 & $\mathbf{4 . 5 5}$ & 6.56 & 12.5
\end{tabular}

TABLE 5.2 Safe Distances at Several Percentiles for Trichlorosilane (UN 1295) Releases into Water as Determined in the ERG2004 Analysis

\begin{tabular}{lccccccc}
\hline & & \multicolumn{6}{c}{ Safe Distance (mi) for Given Percentile } \\
\cline { 3 - 8 } \multirow{2}{*}{ Release Size } & Time & 50 & 70 & 80 & 90 & 95 & 99 \\
\hline \multirow{3}{*}{ Small } & & & & & & & \\
& Day & 0.04 & 0.06 & 0.07 & $\mathbf{0 . 1 0}$ & 0.16 & 0.72 \\
\multirow{4}{*}{ Large } & Night & 0.08 & 0.16 & 0.28 & $\mathbf{0 . 5 9}$ & 0.86 & 1.29 \\
& & & & & & & \\
& Day & 0.22 & 0.49 & 0.83 & $\mathbf{1 . 5 3}$ & 2.62 & 6.51 \\
& Night & 0.81 & 1.71 & 2.45 & $\mathbf{4 . 0 2}$ & 6.36 & 11.55 \\
\hline
\end{tabular}

TABLE 5.3 Safe Distances at Several Percentiles for Landbased Phosphorous Trichloride (UN 1809) Releases as Determined in the ERG2004 Analysis

\begin{tabular}{lccccccc}
\hline & & \multicolumn{6}{c}{ Safe Distance (mi) for Given Percentile } \\
\cline { 3 - 8 } \multirow{2}{*}{ Release Size } & Time & 50 & 70 & 80 & 90 & 95 & 99 \\
\hline \multirow{2}{*}{ Small } & & & & & & & \\
& Day & 0.01 & 0.03 & 0.05 & $\mathbf{0 . 0 7}$ & 0.10 & 0.27 \\
\multirow{3}{*}{ Large } & Night & 0.04 & 0.07 & 0.13 & $\mathbf{0 . 2 2}$ & 0.34 & 0.73 \\
& & & & & & & \\
& Day & 0.32 & 0.54 & 0.71 & $\mathbf{0 . 9 2}$ & 1.41 & 3.46 \\
& Night & 0.67 & 1.17 & 1.57 & $\mathbf{2 . 1 2}$ & 3.26 & 6.56 \\
\hline
\end{tabular}


TABLE 5.4 Safe Distances at Several Percentiles for Ethylene Oxide (UN 1040) Releases as Determined in the ERG2004 Analysis

\begin{tabular}{lcccccccc}
\hline & & \multicolumn{6}{c}{ Safe Distance (mi) for Given Percentile } \\
\cline { 3 - 9 } Release Size & Time & 50 & 70 & 80 & 90 & 95 & 99 \\
\hline \multirow{2}{*}{ Small } & & & & & & & \\
& Day & 0.01 & 0.01 & 0.02 & $\mathbf{0 . 0 2}$ & 0.03 & 0.09 \\
& Night & 0.02 & 0.04 & 0.06 & $\mathbf{0 . 0 8}$ & 0.13 & 0.22 \\
\multirow{2}{*}{ Large } & & & & & & & \\
& Day & 0.11 & 0.21 & 0.39 & $\mathbf{0 . 4 9}$ & 0.81 & 2.04 \\
& Night & 0.36 & 0.79 & 1.04 & $\mathbf{1 . 4 9}$ & 2.80 & 6.00 \\
\hline
\end{tabular}

to worst-case releases in worst-case meteorology, can result in safe distances exceeding the PAD by a factor of $2-4$. However, using the $99^{\text {th }}$-percentile criterion to define the PAD would result in extreme overreaction to the vast majority of incidents first responders face. The $90^{\text {th }}$-percentile criterion was selected to strike a reasonable balance between adequately protecting the public from exposure to potentially harmful substances and avoiding the needless risks and expense associated with overreaction.

\subsection{PRESENTATION OF THE TABLE IN THE GUIDEBOOK}

Appendix A provides the Table of Initial Isolation and Protective Action Distances as it appears in the ERG2004 (DOT et al. 2004) with one important modification discussed below. The PADs in the Table are the $90^{\text {th }}$ percentiles of the safe distance distributions presented in the previous section. As discussed in Section 2, three distinct types of materials are listed in the Table. The first are TIH materials released in transportation-related incidents. The second are water-reactive materials that emit TIH gases when spilled into water. These entries are denoted by the phrase "when spilled in water." For TIH materials that emit a secondary TIH product when spilled in water, two entries are listed; they correspond to spills in water and on land. The third type of materials is chemical warfare agents released in a malicious manner. These entries are denoted by the phrase "when used as a weapon." Some chemical warfare agents, such as arsine and hydrogen cyanide, are TIH industrial chemicals as well and contain additional entries for transportation-related releases.

In all cases, the PADs have been rounded up the next $0.1 \mathrm{mi}$. Appendix A differs from the presentation in the ERG2004 in that distances are not limited to 7 mi (or $11 \mathrm{~km}$ ). These "unclipped" values are provided to more accurately show the relative risk of the high-hazard materials for which PADs can exceed $7 \mathrm{mi}$. Initial Isolation Distances are rounded up to the next $100 \mathrm{ft}$ for distances shorter than 2,000 ft and to the next $500 \mathrm{ft}$ for distances longer than 2,000 ft. Initial Isolation Distances are limited to 3,000 ft. 


\subsection{EXTENSIONS OF THE ERG ANALYSIS}

One key limitation of PAD estimates in the ERG is that the only information taken into account is the spill size (large or small) and whether it is day or night. Clearly, the distributions in Figures 5.1-5.8 show wide variability in PAD estimates, and the data used to construct them contain a great amount of additional information that could be very useful in emergency response situations as well as a wide variety of other analyses. Factors that could be easily ascertained by emergency response personnel are whether they are dealing with a highway or rail incident, whether a vehicular accident or derailment is involved (as opposed to an en route/nonaccident event), general wind conditions (e.g., high or low wind speed), month of year, temperature, and cloud cover (clear or overcast). Each of these factors can affect the safe-distance estimate by a factor of 3 or more.

Examples of additional information that can considerably narrow the safe-distance distributions are shown in Figures 5.9-5.12. Figures 5.9 and 5.10 are for chlorine and are actually subsets of the distribution shown in Figure 5.4. In these figures, the distributions are shown with the ordinate on a $\log$ scale to emphasize differences at higher percentiles. The percentiles of the distributions are shown as circles superimposed on the curves. Figure 5.9 compares safe-distance distributions for large, daytime chlorine spills from rail transported tank cars and highway transported cargo tanks. Since rail cars contain approximately four times the quantity of chlorine as highway vehicles, safe-distance estimates are higher at all percentiles for tank cars than highway cargo tanks. At the $50^{\text {th }}, 70^{\text {th }}, 80^{\text {th }}$, and $90^{\text {th }}$ percentiles, safe distances for rail releases falling into the large spill definition in the ERG ( $>60$ gal) are about double that for corresponding highway cargo tank releases.

Figure 5.10 shows a comparison for two different wind-speed cases representing low winds $(2-3 \mathrm{~m} / \mathrm{s})$ and high winds $(7-9 \mathrm{~m} / \mathrm{s})$, demonstrating the profound influence of wind speed on atmospheric dispersion and, consequently, safe distances. Above the $70^{\text {th }}$ percentile, safe distances for low-wind-speed cases greatly exceed those for high-wind-speed cases. For highwind-speed cases, a 5-mi safe distance would provide protection $99.5 \%$ of the time, whereas it would only be sufficient $65 \%$ of the time for low-wind-speed cases.

Figure 5.11 compares safe-distance distributions for two different times of year. Here, we show results for a volatile liquid, fuming nitric acid. Higher temperatures characteristic of summer increase the pool evaporation rate and therefore increase safe distances. Again, we see about factor-of-2 differences between percentiles for summer and winter cases. These distributions could be refined much further if we consider a particular location rather than the full range of locations in the ERG analysis.

The last example we consider is in Figure 5.12. Here we show the effect of increased information on safe-distance estimates for a rail chlorine spill over 1,000 gal. Each of the factors listed in the figure could be easily determined by personnel arriving at the accident scene. The first curve shown is the large spill daytime distribution illustrated in Figure 5.3. This distribution comprises over 422,451 safe-distance estimates from our statistical analysis. Next, we narrow this to tank car spills over 1,000 gal. These incidents comprise $4.45 \%$ of the original distribution 


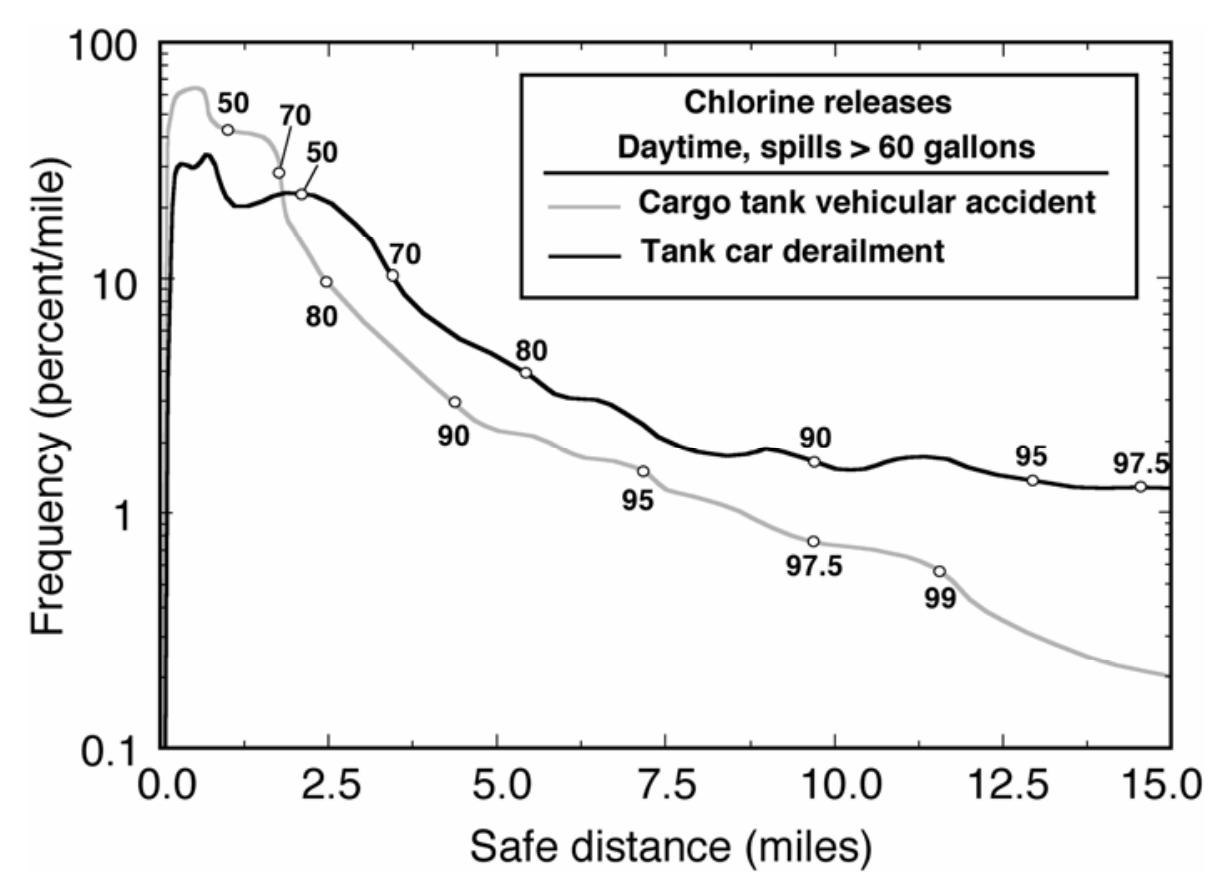

FIGURE 5.9 Frequency of Safe Distances for Large Daytime Chlorine Spills Occurring as a Result of Vehicular Accidents and Tank Car Derailments. Percentiles are denoted by open circles superimposed on the curves. These are a subset of cases shown in Figure 5.3.

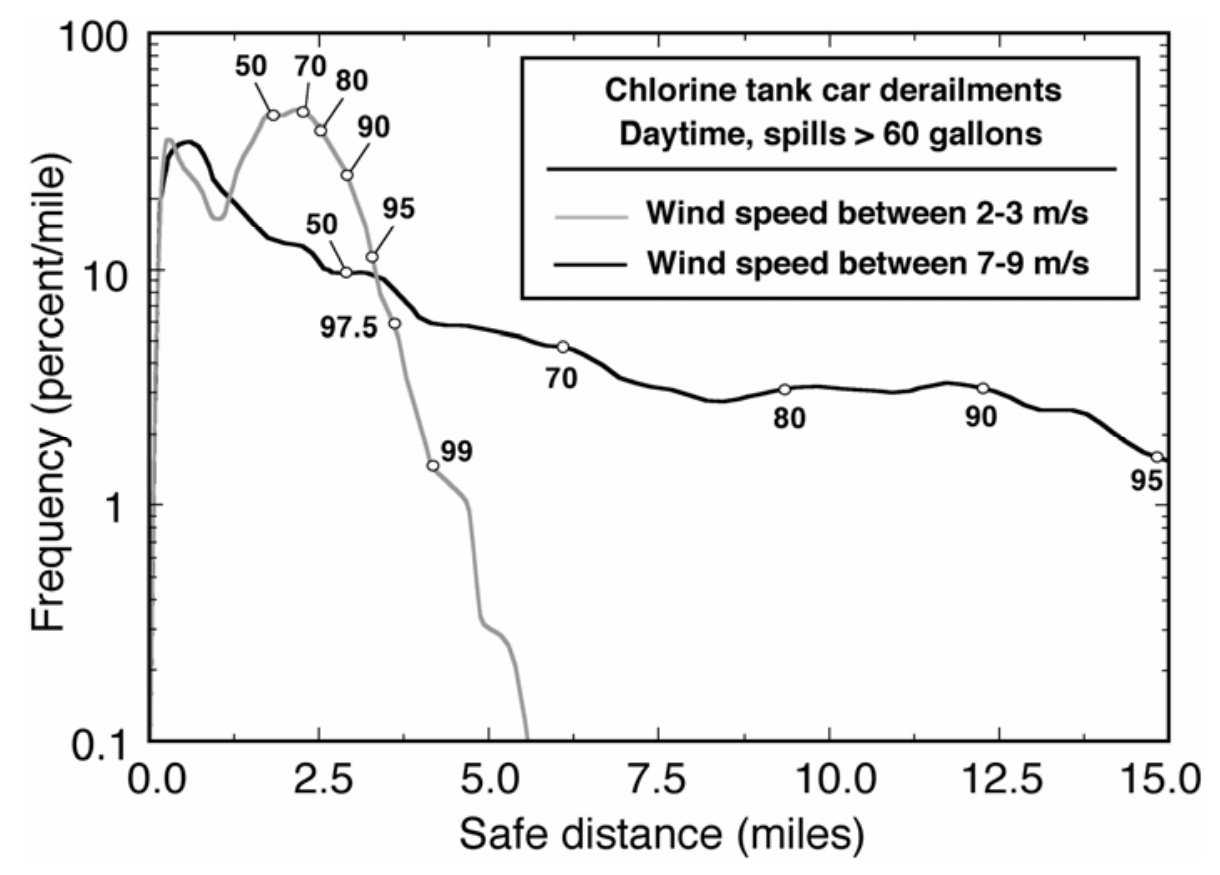

FIGURE 5.10 Frequency of Safe Distances for Large Daytime Chlorine Spills for Different Wind Speed Ranges. Percentiles are denoted by open circles superimposed on the curves. These are a subset of cases shown in Figure 5.9. 


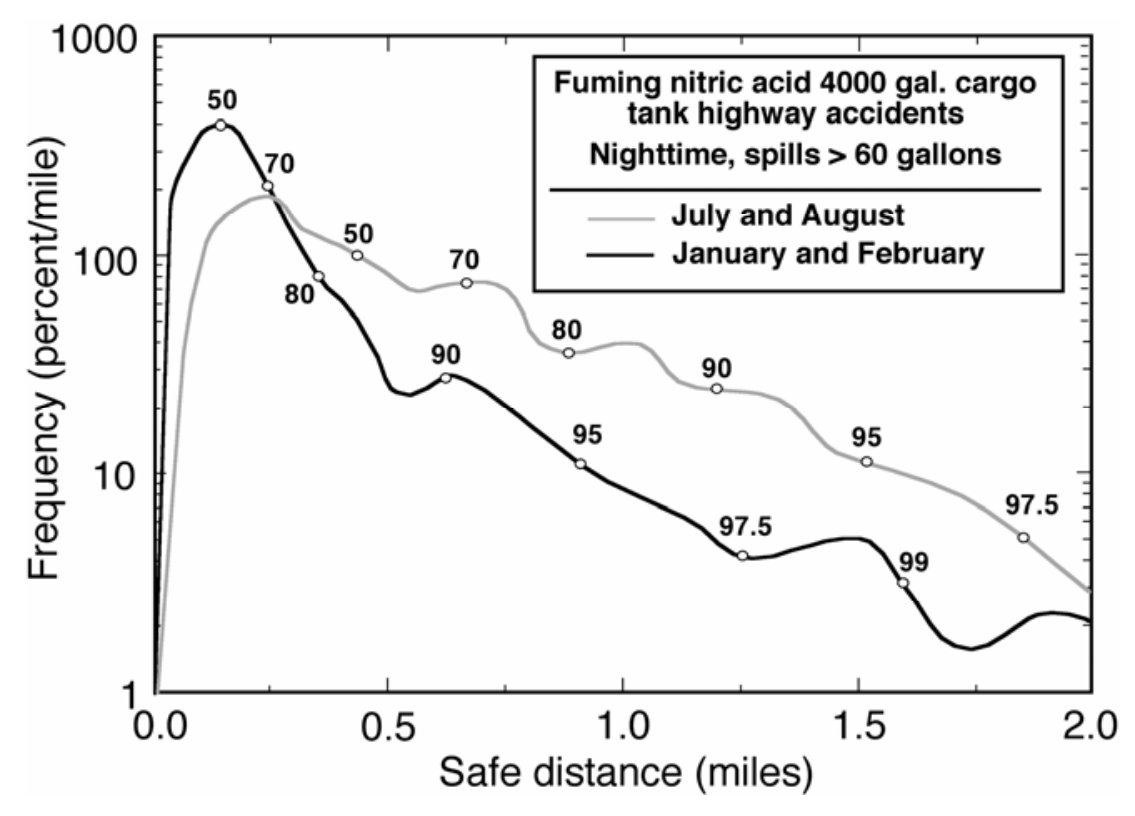

FIGURE 5.11 Frequency of Safe Distances for Highway Accidents Involving Fuming Nitric Acid 4,000-gal Cargo Tanks for Summer and Winter Conditions, Illustrating the Effects of Seasonal Climatology. Percentiles are denoted by open circles superimposed on the curves.

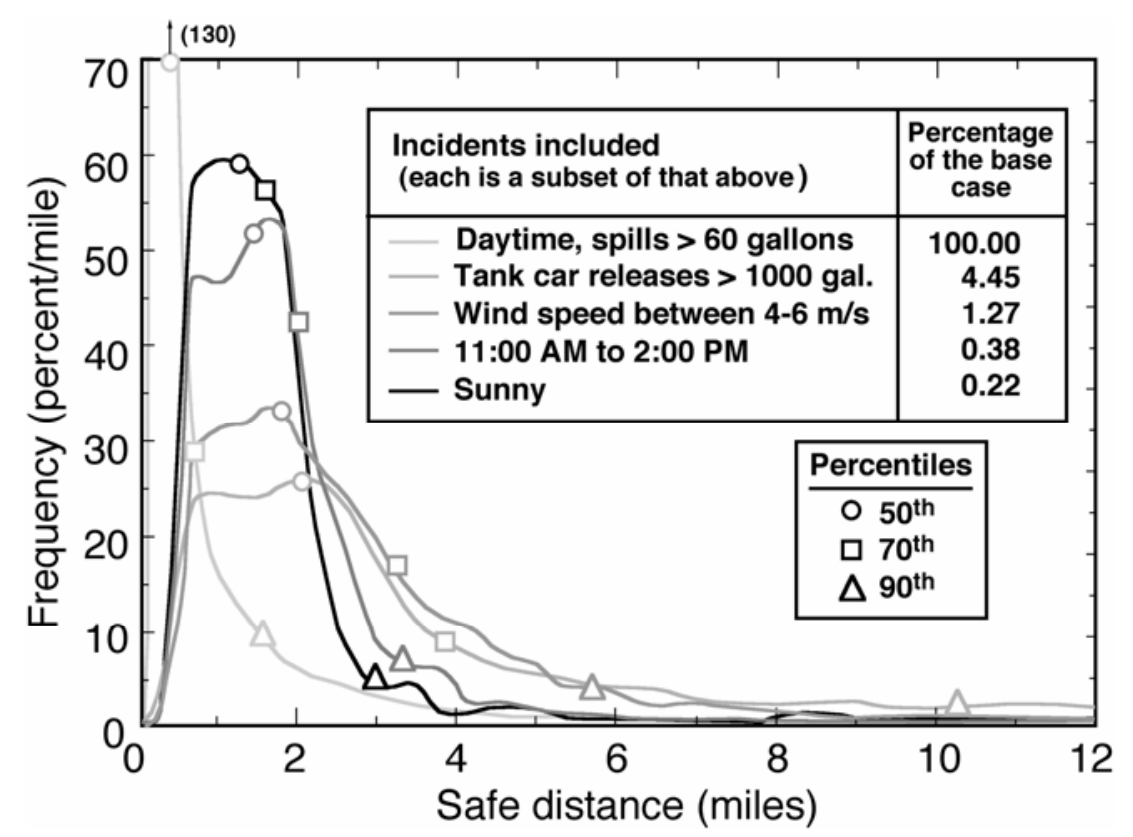

FIGURE 5.12 Frequency of Safe Distances for a Chlorine Tank Car Release Greater than 1,000 gal, Occurring from 11 a.m. to 2 p.m. on a Sunny Day with the Wind Between 4 and $6 \mathrm{~m} / \mathrm{s}$, Showing the Effect on the Safe-distance Distribution as Each Constraint Is Imposed. Percentiles are denoted as shown in the legend. The percentage of the base case (as given in Figure 5.3) is also provided. 
(18,786 cases). These cases are among the worst cases of the original distribution in terms of spill amount, as evidenced by the fact that the $90^{\text {th }}$-percentile value is more than a factor of 5 greater than that for the original distribution. The next three curves refine this distribution using easily determined meteorology and time-of-day variables. The final case is that for a tank car release above 1,000 gal, occurring from 11 a.m. to 2 p.m. on a sunny day with the wind between 4 and $6 \mathrm{~m} / \mathrm{s}$. This combination of conditions represents only $0.22 \%$ of the cases comprising the original distribution for chlorine daytime large spills. 


\section{REFERENCES}

ACGIH, 1995, Threshold Limit Values for Chemical Substances and Physical Agents and Biological Exposure Indices, American Conference of Governmental Industrial Hygienists, Cincinnati, Ohio.

AIHA, 2004, The AIHA 2004 Emergency Response Planning Guidelines and Workplace Environmental Exposure Level Guidelines Handbook, American Industrial Hygiene Association, Fairfax, Va.

Barad, M.L. (editor), 1958, Project Prairie Grass: A Field Program in Diffusion, Vols. 1 and 2, part of Geophysical Research Papers No. 59 series; also Report AFCRL-58-235, Air Force Cambridge Research Center, July.

Brown, D.F., 1997, "Development of an Improved Meteorological Preprocessor and Atmospheric Dispersion Model," Ph.D. thesis, Department of Mechanical and Industrial Engineering, University of Illinois, Urbana, Ill.

Brown, D.F., and W.E. Dunn, 1998, The Surface Energy Budget Meteorological Model (SEBMET) for Atmospheric Boundary Layer Characterization, prepared by University of Illinois, Urbana, Ill., for U.S. Army Corps of Engineers, Construction and Research Laboratory, Champaign, Ill.

Brown, D.F., W.E. Dunn, and A.J. Policastro, 2001, A National Risk Assessment for Selected Materials in Transportation, ANL-DIS-01-1, Argonne National Laboratory, Argonne, Ill.

Budavari, S. (ed.), 1996, The Merck Index, Merck \& Co., Whitehouse Station, N.J.

Calder, K.L., 1949, "Eddy Diffusion and Evaporation in Flow over Aerodynamically Smooth and Rough Surfaces: A Treatment Based on Laboratory Laws of Turbulent Flow with Special Reference to Conditions in the Lower Atmosphere," Quarterly Journal of Mechanical and Applied Math 92:153-176.

Carhart, R.A., et al., 1996, Technical Report Documentation to Support the 1996 North American Emergency Response Guidebook. Part II, Toxic Gases Produced from Reactions of Hazardous Materials Spilled into Natural Waters in Table "List of Dangerous Water-Reactive Materials," prepared by University of Chicago, Chicago, Ill., for Research and Special Projects Administration, Office of Hazardous Materials Technology, U.S. Department of Transportation.

Carson, P.A., and C.J. Mumford, 1994, Hazardous Chemicals Handbook, ButterworthHeinemann, Boston, Mass.

Cleven, R.F.M.J., and M. van Bruggen 2000, The Cyanide Accident in Barskoon, 609026001, Dutch National Institute of Public Health and the Environmenta; available at http://www.rivm.nl/bibliotheek/rapporten/609026001.html. 
Cotton, F.A., and G. Wilkinson, 1966, Advanced Inorganic Chemistry, A Comprehensive Text, $2^{\text {nd }}$ Ed., Interscience Publishers, New York.

Craig, D.K., et al., 1995, "Alternative Guideline Limits for Chemicals without Environmental Response Planning Guidelines," American Industrial Hygiene Association Journal 56:919-925.

DTRA, 1999, HPAC User's Guide (Ver. 3.2), HPAC-UGUIDE-01-U-RACO, prepared by SAIC for Defense Threat Reduction Agency, Alexandria, Va., Oct. 4.

Doe, J.E., and G.M. Milburn, 1983, "The Relationship between Exposure Concentration, Duration of Exposure and Inhalation LC 50 Values," Human Toxicology 2:564.

DOT et al., 2004, 2004 Emergency Response Guidebook, Prepared by J.J. Keller \& Associates, Inc., Neenah, Wis., for U.S. Department of Transportation, Transport Canada, and Secretariat of Transport and Communications of Mexico. (Referred to in this document as ERG2004.)

Draxler, R.R., 1976, "Determination of Atmospheric Diffusion Parameters," Atmospheric Environment 10:99-105.

Driedonks, A.G.M., 1982, "Models and Observations of the Growth of the Atmospheric Boundary Layer," Boundary Layer Meteorology 23:283-306.

EPA, 1991, Technical Guidance for Hazards Analysis, Emergency Planning for Extremely Hazardous Substances, EPA-OSWER-88-0001, U.S. Environmental Protection Agency, Washington, D.C.

EPA, 1992, Workbook of Screening Techniques for Assessing Impacts of Toxic Air Pollutants (Revised), EPA-454/R-92-024, U.S. Environmental Protection Agency, Washington, D.C., Dec.

EPA, 1996, Offsite Consequence Analysis Guidance, Chemical Emergency Preparedness and Prevention Office, U.S. Environmental Protection Agency, Washington, D.C.

EPA and OSHA, 1997, EPA/OHSA Joint Chemical Accident Investigation Report, Napp Technologies, Inc., Lodi, NJ, EPA-550-R-97-002, U.S. Environmental Protection Agency, Washington, D.C., Oct.

Dunn, W.E., et al., 1996, Technical Documentation in Support of the 1996 North American Emergency Response Guidebook, prepared by University of Illinois, Urbana, Ill., for Research and Special Projects Administration, Office of Hazardous Materials Technology, U.S. Department of Transportation.

EPA: See U.S. Environmental Protection Agency. 
Ermak, D.L., 1990, User's Manual for SLAB: An Atmospheric Dispersion Model for DenserThan-Air Releases, ACRL-MA-105607, Lawrence Livermore National Laboratory, Livermore, Calif.

Frawley, J.P., et al., 1964, "Emergency Exposure Limits," American Industrial Hygiene Association Journal 25:578-586.

Gryning, S.W., and E. Lyck, 1984, "Atmospheric Dispersion from Elevated Sources in an Urban Area: Comparison between Tracer Experiments and Model Calculations," Journal of Climate and Applied Meteorology 23:651-660.

Gryning, S., et al., 1983, "Dispersion from a Continuous Ground-Level Source Investigated by a K Model," Quarterly Journal of the Royal Meteorological Society 109:355-364.

Haber, F., 1924, Funf Vortrage Aus Den Jaren 1920-1923, Springer-Verlage, Berlin, Germany.

Hanna, S.R., and J.C. Chang, 1992, "Boundary Layer Parameterizations for Applied Dispersion Modeling over Urban Areas," Boundary Layer Meteorology 58:229-259.

Hanna, S.R., and R.J. Paine, 1989, "Hybrid Plume Dispersion Model (HDPM) Development and Evaluation," Journal of Applied Meteorology 28:206-224.

Havens, J., 1988, A Dispersion Model for Elevated Dense Gas Jet Chemical Releases, Volume I and II, EPA-450/4-88-006B, U.S. Environmental Protection Agency, Apr.

Iqbal, M., 1983, An Introduction to Solar Radiation, Academic Press, Toronto, Ontario, Canada.

Irwin, J.S., 1983, "Estimating Plume Dispersion - A Comparison of Several Sigma Schemes," Journal of Climate and Applied Meteorology 22:92-114.

Johnson, D.W., and J.L. Woodward JL, 1999, RELEASE: A Model with Data to Predict Aerosol Rainout in Accidental Releases, American Institute of Chemical Engineers, Center for Chemical Process Safety, New York.

Kapias, T., and R.F. Griffiths, 1999, “A New Model for Accidental Releases of Water Reactive Chemicals," Journal of Hazardous Materials 63:133-143.

Klimisch, H.J., et al., 1987, "Classification of Dangerous Substances and Pesticides in the European Economic Community Directives: A Proposed Revision of Criteria for Inhalation Toxicity," Regulatory Toxicology and Pharmacology 7:21-34.

Kroschwitz, J. (editor), 1991-1996, Kirk-Othmer's Encyclopedia of Chemical Technology, $4^{\text {th }}$ ed., Wiley-Interscience, New York.

Lesht, B.M., and D.J. Brandner, 1992, "Functional Representation of Great Lakes Surface Temperatures," Journal of Great Lakes Research 18(1):98-107. 
Lewis, R.J. (ed.), 1966, Sax’s Dangerous Properties of Industrial Materials, 9th Ed., Van Nostrand Reinhold, New York.

Lewis, R.J. (ed.), 1989, Hawley's Condensed Chemical Dictionary, 9th Ed., Van Nostrand Reinhold, New York.

Lewis, R.J. (ed.), 1993, Hazardous Chemicals Desk Reference, $3^{\text {rd }}$ Ed., Van Nostrand Reinhold, New York.

Lewis, R.J., and N.I. Sax, 1992, Dangerous Properties of Industrial Materials, $8^{\text {th }}$ Ed., Van Nostrand Reinhold, New York.

NREL, 1992, User's Manual: National Solar Radiation Data Base, prepared by National Renewable Energy Laboratory, Golden, Colo., with data distributed by National Climatic Data Center, Asheville, N.C.

NRC, 1984-1987, Emergency and Continuous Exposure Guidance Levels for Selected Airborne Contaminants, Vol. I-VII, Committee on Toxicology, Board on Toxicology and Environmental Health Hazards, Commission on Life Sciences, National Research Council, Washington, D.C.

Nieuwstadt, F.T.M., 1981, "The Steady-State Height and Resistance Laws of the Nocturnal Boundary Layer: Theory Compared with Cabauw Observations," Boundary Layer Meteorology 20:3-17.

NIOSH, 1994, Documentation for Immediately Dangerous to Life or Health Concentrations, U.S. Department of Health and Human Services, Public Health Service, Centers for Disease Control and Prevention and National Institute for Occupational Safety and Health, Cincinnati, Ohio.

OSHA, 1989, "Permissible Exposure Limits, Title 29 of the Code of Federal Regulations Part 1910.1000," Federal Register, U.S. Department of Labor, Occupational Safety and Health Administration, Jan. 19.

Panofsky, H.A., and J.A. Dutton, 1984, Atmospheric Turbulence - Models and Methods for Engineering Applications, John Wiley and Sons, New York.

Panofsky, H.A., et al., 1977, "The Characteristics of Turbulent Velocity Components in the Surface Layer under Convective Conditions," Boundary Layer Meteorology 11:355-361.

Perry, R.H., et al. (editors), 1984, Perry's Chemical Engineers Handbook, $6^{\text {th }}$ Ed., McGraw-Hill, New York.

Post, L., 1994, HGSystem 3.0 Technical Reference Manual, TNER.94.059, Shell Research Limited, Thornton Research Centre, Chester, England. 
Raj, P.K., 1985, "Summary of Heavy Gas Spills Modeling Research," pp. 51-75 in Proceedings of Heavy Gas (LNG/LPG) Workshop, Toronto, Ontario, Canada.

Raj, P.K., and C.K. Turner, 1993, Hazardous Materials Transportation in Tank Cars: Analysis of Risks Part 1, DOT/FRA/ORD-92/34, prepared by Technology \& Management Systems, Inc., Burlington, Mass., for the Federal Railroad Administration, Washington, D.C.

Reynolds, M., 1992, ALOHA (Area Locations of Hazardous Atmospheres) 5.0 Technical Description, NOS ORCA-65, National Oceanic and Atmospheric Administration, Seattle, Wash.

Roberts, O.F.T., 1923, "The Theoretical Scattering of Smoke in a Turbulent Atmosphere," pp. 640-654 in Proceedings of the Royal Society of London A 104.

Sykes R.I., S.F, Parker, D.S. Henn, C.P. Cerasoli, and L.P. Santos, 1998, PC-SCIPUFF Version 1.2PD Technical Documentation. ARAP 718, Titan Corporation, Princeton, N.J.

Taylor, G.I., 1921, "Diffusion by Continuous Movements," pp. 196-212 in Proceedings of the London Math Society 20.

Ten Berge, W.F., et al., 1986, "Concentration-Time Mortality Response Relationship of Irritant and Systemically Acting Vapors and Gases," Journal of Hazardous Materials 13:301-309.

Turner, D., and S. Fairhurst, 1989, Assessment of the Toxicity of Major Hazard Substances, Report 21, Health and Safety Executive, London, England.

van Ulden, A.P., 1978, "Simple Estimates for Vertical Diffusion from Sources near the Ground," Atmospheric Environment 12:2125-2129.

Willis, G.E., and J.W. Deardorff, 1976, “A Laboratory Model of Diffusion into the Convective Planetary Boundary Layer," Quarterly Journal of the Royal Meteorological Society 102:427445 .

Woudenberg, F., and P. Van Der Torn, 1992, "Emergency Exposure Limits: A Guide To Quality Assurance and Safety," Quality Assurance: Good Practice, Regulation, and Law 1:249-293. 


\section{APPENDIX A:}

TABLE OF INITIAL ISOLATION AND PROTECTIVE ACTION DISTANCES IN THE 2004 EMERGENCY RESPONSE GUIDEBOOK 


\section{APPENDIX A:}

\section{TABLE OF INITIAL ISOLATION AND PROTECTIVE ACTION DISTANCES IN THE 2004 EMERGENCY RESPONSE GUIDEBOOK}

Table A.1 provides the Table of Initial Isolation and Protective Action Distances for the 2004 Emergency Response Guidebook (in English units only). Table values are identical to those appearing in ERG2004, except that distances are not truncated at $7 \mathrm{mi}(11 \mathrm{~km})$. 
TABLE A.1 Table of Initial Isolation and Protective Action Distances

\begin{tabular}{|c|c|c|c|c|c|c|c|}
\hline \multirow[b]{3}{*}{$\begin{array}{l}\text { UN ID } \\
\text { No. }\end{array}$} & \multirow[b]{3}{*}{ Name of Material } & \multicolumn{3}{|c|}{ Small Spills } & \multicolumn{3}{|c|}{ Large Spills } \\
\hline & & \multirow{2}{*}{$\begin{array}{c}\text { First } \\
\text { Isolate } \\
\text { in All } \\
\text { Direc- } \\
\text { tions (ft) }\end{array}$} & \multicolumn{2}{|c|}{$\begin{array}{l}\text { Then Protect } \\
\text { Persons } \\
\text { Downwind } \\
\text { during }\end{array}$} & \multirow{2}{*}{$\begin{array}{c}\text { First } \\
\text { Isolate } \\
\text { in All } \\
\text { Direc- } \\
\text { tions (ft) }\end{array}$} & \multicolumn{2}{|c|}{$\begin{array}{l}\text { Then Protect } \\
\text { Persons } \\
\text { Downwind } \\
\text { during }\end{array}$} \\
\hline & & & $\begin{array}{l}\text { Day } \\
(\mathrm{mi})\end{array}$ & $\begin{array}{l}\text { Night } \\
(\mathrm{mi})\end{array}$ & & $\begin{array}{l}\text { Day } \\
(\mathrm{mi})\end{array}$ & $\begin{array}{l}\text { Night } \\
(\mathrm{mi})\end{array}$ \\
\hline 1005 & Ammonia, anhydrous & 100 & 0.1 & 0.1 & 200 & 0.4 & 1.4 \\
\hline 1005 & Ammonia, anhydrous, liquefied & 100 & 0.1 & 0.1 & 200 & 0.4 & 1.4 \\
\hline 1005 & Ammonia, solution, with more than $50 \%$ ammonia & 100 & 0.1 & 0.1 & 200 & 0.4 & 1.4 \\
\hline 1005 & Anhydrous ammonia & 100 & 0.1 & 0.1 & 200 & 0.4 & 1.4 \\
\hline 1005 & Anhydrous ammonia, liquefied & 100 & 0.1 & 0.1 & 200 & 0.4 & 1.4 \\
\hline 1008 & Boron trifluoride & 100 & 0.1 & 0.4 & 600 & 1.1 & 3.0 \\
\hline 1008 & Boron trifluoride, compressed & 100 & 0.1 & 0.4 & 600 & 1.1 & 3.0 \\
\hline 1016 & Carbon monoxide & 100 & 0.1 & 0.1 & 300 & 0.4 & 1.5 \\
\hline 1016 & Carbon monoxide, compressed & 100 & 0.1 & 0.1 & 300 & 0.4 & 1.5 \\
\hline 1017 & Chlorine & 100 & 0.2 & 0.8 & 800 & 1.5 & 4.6 \\
\hline 1023 & Coal gas & 100 & 0.1 & 0.1 & 200 & 0.2 & 0.3 \\
\hline 1023 & Coal gas, compressed & 100 & 0.1 & 0.1 & 200 & 0.2 & 0.3 \\
\hline 1026 & Cyanogen & 100 & 0.2 & 0.8 & 400 & 0.7 & 2.7 \\
\hline 1026 & Cyanogen, liquefied & 100 & 0.2 & 0.8 & 400 & 0.7 & 2.7 \\
\hline 1026 & Cyanogen gas & 100 & 0.2 & 0.8 & 400 & 0.7 & 2.7 \\
\hline 1040 & Ethylene oxide & 100 & 0.1 & 0.1 & 300 & 0.5 & 1.5 \\
\hline 1040 & Ethylene oxide with Nitrogen & 100 & 0.1 & 0.1 & 300 & 0.5 & 1.5 \\
\hline 1045 & Fluorine & 100 & 0.1 & 0.3 & 300 & 0.5 & 2.2 \\
\hline 1045 & Fluorine, compressed & 100 & 0.1 & 0.3 & 300 & 0.5 & 2.2 \\
\hline 1048 & Hydrogen bromide, anhydrous & 100 & 0.1 & 0.3 & 600 & 1.1 & 3.6 \\
\hline 1050 & Hydrogen chloride, anhydrous & 100 & 0.1 & 0.3 & 1,200 & 2.2 & 6.5 \\
\hline 1051 & $\mathrm{AC}$ (when used as a weapon) & 200 & 0.1 & 0.3 & 1,500 & 1.0 & 2.4 \\
\hline 1051 & $\begin{array}{l}\text { Hydrocyanic acid, aqueous solutions, with more than } 20 \% \\
\text { Hydrogen cyanide }\end{array}$ & 100 & 0.1 & 0.3 & 500 & 0.8 & 2.3 \\
\hline 1051 & Hydrocyanic acid, liquefied & 100 & 0.1 & 0.3 & 500 & 0.8 & 2.3 \\
\hline 1051 & Hydrogen cyanide, anhydrous, stabilized & 100 & 0.1 & 0.3 & 500 & 0.8 & 2.3 \\
\hline 1051 & Hydrogen cyanide, stabilized & 100 & 0.1 & 0.3 & 500 & 0.8 & 2.3 \\
\hline 1052 & Hydrogen fluoride, anhydrous & 100 & 0.1 & 0.3 & 700 & 1.2 & 2.7 \\
\hline 1053 & Hydrogen sulfide & 100 & 0.1 & 0.2 & 700 & 1.3 & 3.9 \\
\hline 1053 & Hydrogen sulfide, liquefied & 100 & 0.1 & 0.2 & 700 & 1.3 & 3.9 \\
\hline 1053 & Hydrogen sulphide & 100 & 0.1 & 0.2 & 700 & 1.3 & 3.9 \\
\hline 1053 & Hydrogen sulphide, liquefied & 100 & 0.1 & 0.2 & 700 & 1.3 & 3.9 \\
\hline 1062 & Methyl bromide & 100 & 0.1 & 0.1 & 300 & 0.5 & 1.4 \\
\hline 1064 & Methyl mercaptan & 100 & 0.1 & 0.2 & 500 & 0.8 & 2.8 \\
\hline 1067 & Dinitrogen tetroxide & 100 & 0.1 & 0.3 & 500 & 1.0 & 2.5 \\
\hline 1067 & Dinitrogen tetroxide, liquefied & 100 & 0.1 & 0.3 & 500 & 1.0 & 2.5 \\
\hline 1067 & Nitrogen dioxide & 100 & 0.1 & 0.3 & 500 & 1.0 & 2.5 \\
\hline 1067 & Nitrogen dioxide, liquefied & 100 & 0.1 & 0.3 & 500 & 1.0 & 2.5 \\
\hline 1069 & Nitrosyl chloride & 100 & 0.1 & 0.6 & 1,500 & 2.7 & 6.9 \\
\hline 1071 & Oil gas & 100 & 0.1 & 0.1 & 200 & 0.2 & 0.3 \\
\hline
\end{tabular}


TABLE A.1 (Cont.)

\begin{tabular}{|c|c|c|c|c|c|c|c|}
\hline \multirow[b]{3}{*}{$\begin{array}{l}\text { UN ID } \\
\text { No. }\end{array}$} & \multirow[b]{3}{*}{ Name of Material } & \multicolumn{3}{|c|}{ Small Spills } & \multicolumn{3}{|c|}{ Large Spills } \\
\hline & & \multirow{2}{*}{$\begin{array}{c}\text { First } \\
\text { Isolate } \\
\text { in All } \\
\text { Direc- } \\
\text { tions (ft) }\end{array}$} & \multicolumn{2}{|c|}{$\begin{array}{l}\text { Then Protect } \\
\text { Persons } \\
\text { Downwind } \\
\text { during }\end{array}$} & \multirow{2}{*}{$\begin{array}{c}\text { First } \\
\text { Isolate } \\
\text { in All } \\
\text { Direc- } \\
\text { tions (ft) }\end{array}$} & \multicolumn{2}{|c|}{$\begin{array}{l}\text { Then Protect } \\
\text { Persons } \\
\text { Downwind } \\
\text { during }\end{array}$} \\
\hline & & & $\begin{array}{l}\text { Day } \\
\text { (mi) }\end{array}$ & $\begin{array}{l}\text { Night } \\
(\mathrm{mi})\end{array}$ & & $\begin{array}{l}\text { Day } \\
\text { (mi) }\end{array}$ & $\begin{array}{l}\text { Night } \\
(\mathrm{mi})\end{array}$ \\
\hline 1071 & Oil gas, compressed & 100 & 0.1 & 0.1 & 200 & 0.2 & 0.3 \\
\hline 1076 & $\mathrm{CG}$ (when used as a weapon) & 500 & 0.8 & 2.0 & 2,500 & 4.5 & 7.1 \\
\hline 1076 & Diphosgene & 300 & 0.6 & 2.6 & 2,500 & 4.1 & 9.1 \\
\hline 1076 & DP (when used as a weapon) & 200 & 0.2 & 0.6 & 600 & 1.0 & 2.8 \\
\hline 1076 & Phosgene & 300 & 0.6 & 2.6 & 2,500 & 4.1 & 9.1 \\
\hline 1079 & Sulfur dioxide & 100 & 0.2 & 0.8 & 700 & 1.3 & 3.9 \\
\hline 1079 & Sulfur dioxide, liquefied & 100 & 0.2 & 0.8 & 700 & 1.3 & 3.9 \\
\hline 1079 & Sulphur dioxide & 100 & 0.2 & 0.8 & 700 & 1.3 & 3.9 \\
\hline 1079 & Sulphur dioxide, liquefied & 100 & 0.2 & 0.8 & 700 & 1.3 & 3.9 \\
\hline 1082 & Trifluorochloroethylene & 100 & 0.1 & 0.1 & 200 & 0.3 & 0.5 \\
\hline 1082 & Trifluorochloroethylene, inhibited & 100 & 0.1 & 0.1 & 200 & 0.3 & 0.5 \\
\hline 1082 & Trifluorochloroethylene, stabilized & 100 & 0.1 & 0.1 & 200 & 0.3 & 0.5 \\
\hline 1092 & Acrolein, inhibited & 200 & 0.3 & 1.1 & 1,600 & 3.0 & 6.3 \\
\hline 1092 & Acrolein, stabilized & 200 & 0.3 & 1.1 & 1,600 & 3.0 & 6.3 \\
\hline 1098 & Allyl alcohol & 100 & 0.1 & 0.1 & 200 & 0.2 & 0.4 \\
\hline 1135 & Ethylene chlorohydrin & 100 & 0.1 & 0.2 & 300 & 0.5 & 1.0 \\
\hline 1143 & Crotonaldehyde, inhibited & 100 & 0.1 & 0.1 & 200 & 0.3 & 0.5 \\
\hline 1143 & Crotonaldehyde, stabilized & 100 & 0.1 & 0.1 & 200 & 0.3 & 0.5 \\
\hline 1162 & Dimethdichlorosilane (when spilled in water) & 100 & 0.2 & 0.7 & 1,000 & 1.9 & 4.9 \\
\hline 1163 & 1,1-Dimethylhydrazine & 100 & 0.1 & 0.1 & 200 & 0.4 & 0.8 \\
\hline 1163 & Dimethylhydrazine, unsymmetrical & 100 & 0.1 & 0.1 & 200 & 0.4 & 0.8 \\
\hline 1182 & Ethyl chloroformate & 100 & 0.1 & 0.2 & 300 & 0.6 & 1.1 \\
\hline 1185 & Ethyleneimine, inhibited & 100 & 0.2 & 0.5 & 600 & 1.2 & 2.5 \\
\hline 1185 & Ethyleneimine, stabilized & 100 & 0.2 & 0.5 & 600 & 1.2 & 2.5 \\
\hline 1196 & Ethyltrichlorosilane (when spilled in water) & 100 & 0.2 & 0.7 & 1,000 & 1.9 & 4.9 \\
\hline 1238 & Methyl chloroformate & 100 & 0.2 & 0.5 & 600 & 1.1 & 2.4 \\
\hline 1239 & Methyl chloromethyl ether & 100 & 0.2 & 0.6 & 900 & 1.6 & 3.5 \\
\hline 1242 & Methyldichlorosilane (when spilled in water) & 100 & 0.1 & 0.4 & 600 & 1.0 & 3.0 \\
\hline 1244 & Methylhydrazine & 100 & 0.2 & 0.3 & 500 & 0.9 & 1.8 \\
\hline 1250 & Methyltrichlorosilane (when spilled in water) & 100 & 0.1 & 0.3 & 500 & 0.8 & 2.5 \\
\hline 1251 & Methyl vinyl ketone & 500 & 0.8 & 2.1 & 3,000 & 9.9 & 12.4 \\
\hline 1251 & Methyl vinyl ketone, stabilized & 500 & 0.8 & 2.1 & 3,000 & 9.9 & 12.4 \\
\hline 1259 & Nickel carbonyl & 300 & 0.5 & 2.2 & 1,600 & 2.9 & 6.1 \\
\hline 1295 & Trichlorosilane (when spilled in water) & 100 & 0.1 & 0.6 & 900 & 1.6 & 4.1 \\
\hline 1298 & Trimethchlorosilane (when spilled in water) & 100 & 0.1 & 0.2 & 300 & 0.5 & 1.7 \\
\hline 1305 & Vinyltrichlorosilane (when spilled in water) & 100 & 0.1 & 0.5 & 600 & 1.1 & 3.1 \\
\hline 1305 & Vinyltrichlorosilane, inhibited (when spilled in water) & 100 & 0.1 & 0.5 & 600 & 1.1 & 3.1 \\
\hline 1305 & Vinyltrichlorosilane, stabilized (when spilled in water) & 100 & 0.1 & 0.5 & 600 & 1.1 & 3.1 \\
\hline 1340 & $\begin{array}{l}\text { Phosphorus pentasulfide, free from yellow or white } \\
\text { Phosphorous (when spilled in water) }\end{array}$ & 100 & 0.1 & 0.4 & 500 & 0.6 & 2.4 \\
\hline
\end{tabular}


TABLE A.1 (Cont.)

\begin{tabular}{|c|c|c|c|c|c|c|c|}
\hline \multirow[b]{3}{*}{$\begin{array}{l}\text { UN ID } \\
\text { No. }\end{array}$} & \multirow[b]{3}{*}{ Name of Material } & \multicolumn{3}{|c|}{ Small Spills } & \multicolumn{3}{|c|}{ Large Spills } \\
\hline & & \multirow{2}{*}{$\begin{array}{c}\text { First } \\
\text { Isolate } \\
\text { in All } \\
\text { Direc- } \\
\text { tions (ft) }\end{array}$} & \multicolumn{2}{|c|}{$\begin{array}{c}\text { Then Protect } \\
\text { Persons } \\
\text { Downwind } \\
\text { during }\end{array}$} & \multirow{2}{*}{$\begin{array}{c}\text { First } \\
\text { Isolate } \\
\text { in All } \\
\text { Direc- } \\
\text { tions (ft) } \\
\end{array}$} & \multicolumn{2}{|c|}{$\begin{array}{l}\text { Then Protect } \\
\text { Persons } \\
\text { Downwind } \\
\text { during }\end{array}$} \\
\hline & & & $\begin{array}{l}\text { Day } \\
(\mathrm{mi})\end{array}$ & $\begin{array}{c}\text { Night } \\
(\mathrm{mi})\end{array}$ & & $\begin{array}{l}\text { Day } \\
(\mathrm{mi})\end{array}$ & $\begin{array}{c}\text { Night } \\
(\mathrm{mi})\end{array}$ \\
\hline 1340 & $\begin{array}{l}\text { Phosphorus pentasulphide, free from yellow or white } \\
\text { Phosphorous (when spilled in water) }\end{array}$ & 100 & 0.1 & 0.4 & 500 & 0.6 & 2.4 \\
\hline 1360 & Calcium phosphide (when spilled in water) & 200 & 0.3 & 1.3 & 2,500 & 3.9 & 10.1 \\
\hline 1380 & Pentaborane & 300 & 0.6 & 2.1 & 1,800 & 3.3 & 6.9 \\
\hline 1384 & Sodium dithionite (when spilled in water) & 100 & 0.1 & 0.1 & 200 & 0.3 & 0.8 \\
\hline 1384 & Sodium hydrosulfite (when spilled in water) & 100 & 0.1 & 0.1 & 200 & 0.3 & 0.8 \\
\hline 1384 & Sodium hydrosulphite (when spilled in water) & 100 & 0.1 & 0.1 & 200 & 0.3 & 0.8 \\
\hline 1397 & Aluminum phosphide (when spilled in water) & 300 & 0.4 & 1.7 & 3,000 & 5.6 & 12.2 \\
\hline 1412 & Lithium amide (when spilled in water) & 100 & 0.1 & 0.2 & 100 & 0.2 & 1.0 \\
\hline 1419 & Magnesium aluminum phosphide (when spilled in water) & 200 & 0.4 & 1.6 & 3,000 & 4.9 & 11.5 \\
\hline 1432 & Sodium phosphide (when spilled in water) & 200 & 0.2 & 1.1 & 1,600 & 2.9 & 8.5 \\
\hline 1510 & Tetranitromethane & 100 & 0.2 & 0.4 & 300 & 0.5 & 1.0 \\
\hline 1541 & Acetone cyanohydrin (when spilled in water) & 100 & 0.1 & 0.2 & 800 & 0.5 & 1.9 \\
\hline 1556 & MD (when used as a weapon) ${ }^{\mathrm{a}}$ & 100 & 0.2 & 0.5 & 400 & 0.8 & 2.2 \\
\hline 1556 & Methyldichloroarsine & 100 & 0.1 & 0.2 & 200 & 0.4 & 0.7 \\
\hline 1556 & $\mathrm{PD}$ (when used as a weapon) & 100 & 0.1 & 0.1 & 100 & 0.1 & 0.2 \\
\hline 1560 & Arsenic chloride & 100 & 0.2 & 0.2 & 300 & 0.6 & 1.1 \\
\hline 1560 & Arsenic trichloride & 100 & 0.2 & 0.2 & 300 & 0.6 & 1.1 \\
\hline 1569 & Bromoacetone & 100 & 0.1 & 0.4 & 300 & 0.5 & 1.5 \\
\hline 1580 & Chloropicrin & 200 & 0.3 & 0.5 & 700 & 1.2 & 2.2 \\
\hline 1581 & Chloropicrin and Methyl bromide mixture & 100 & 0.1 & 0.4 & 700 & 1.3 & 3.7 \\
\hline 1581 & Methyl bromide and Chloropicrin mixture & 100 & 0.1 & 0.4 & 700 & 1.3 & 3.7 \\
\hline 1582 & Chloropicrin and Methyl chloride mixture & 100 & 0.1 & 0.3 & 100 & 0.2 & 1.1 \\
\hline 1582 & Methyl chloride and Chloropicrin mixture & 100 & 0.1 & 0.3 & 100 & 0.2 & 1.1 \\
\hline 1583 & Chloropicrin mixture, n.o.s. & 200 & 0.3 & 0.5 & 700 & 1.2 & 2.2 \\
\hline 1589 & CK (when used as a weapon) & 200 & 0.4 & 1.5 & 1,300 & 2.5 & 5.0 \\
\hline 1589 & Cyanogen chloride, inhibited & 200 & 0.4 & 1.8 & 1,400 & 2.7 & 6.3 \\
\hline 1589 & Cyanogen chloride, stabilized & 200 & 0.4 & 1.8 & 1,400 & 2.7 & 6.3 \\
\hline 1595 & Dimethyl sulfate & 100 & 0.1 & 0.1 & 200 & 0.3 & 0.5 \\
\hline 1595 & Dimethyl sulphate & 100 & 0.1 & 0.1 & 200 & 0.3 & 0.5 \\
\hline 1605 & Ethylene dibromide & 100 & 0.1 & 0.1 & 100 & 0.2 & 0.4 \\
\hline 1612 & Hexaethyl tetraphosphate and compressed gas mixture & 300 & 0.5 & 1.7 & 1,200 & 2.2 & 5.1 \\
\hline 1613 & $\begin{array}{l}\text { Hydrocyanic acid, aqueous solution, with not more than } 20 \% \\
\text { Hydrogen cyanide }\end{array}$ & 100 & 0.1 & 0.1 & 400 & 0.3 & 0.8 \\
\hline 1613 & $\begin{array}{l}\text { Hydrogen cyanide, aqueous solution, with not more than } \\
20 \% \text { Hydrogen cyanide }\end{array}$ & 100 & 0.1 & 0.1 & 400 & 0.3 & 0.8 \\
\hline 1614 & Hydrogen cyanide, anhydrous, stabilized (absorbed) & 100 & 0.1 & 0.4 & 200 & 0.3 & 1.1 \\
\hline 1614 & Hydrogen cyanide, stabilized (absorbed) & 100 & 0.1 & 0.4 & 200 & 0.3 & 1.1 \\
\hline 1647 & Ethylene dibromide and Methyl bromide mixture, liquid & 100 & 0.1 & 0.1 & 100 & 0.2 & 0.4 \\
\hline 1647 & Methyl bromide and Ethylene dibromide mixture, liquid & 100 & 0.1 & 0.1 & 100 & 0.2 & 0.4 \\
\hline
\end{tabular}


TABLE A.1 (Cont.)

\begin{tabular}{|c|c|c|c|c|c|c|c|}
\hline \multirow[b]{3}{*}{$\begin{array}{l}\text { UN ID } \\
\text { No. }\end{array}$} & \multirow[b]{3}{*}{ Name of Material } & \multicolumn{3}{|c|}{ Small Spills } & \multicolumn{3}{|c|}{ Large Spills } \\
\hline & & \multirow{2}{*}{$\begin{array}{c}\text { First } \\
\text { Isolate } \\
\text { in All } \\
\text { Direc- } \\
\text { tions (ft) }\end{array}$} & \multicolumn{2}{|c|}{$\begin{array}{l}\text { Then Protect } \\
\text { Persons } \\
\text { Downwind } \\
\text { during }\end{array}$} & \multirow{2}{*}{$\begin{array}{c}\text { First } \\
\text { Isolate } \\
\text { in All } \\
\text { Direc- } \\
\text { tions (ft) }\end{array}$} & \multicolumn{2}{|c|}{$\begin{array}{l}\text { Then Protect } \\
\text { Persons } \\
\text { Downwind } \\
\text { during }\end{array}$} \\
\hline & & & $\begin{array}{l}\text { Day } \\
(\mathrm{mi})\end{array}$ & $\begin{array}{l}\text { Night } \\
\text { (mi) }\end{array}$ & & $\begin{array}{l}\text { Day } \\
(\mathrm{mi})\end{array}$ & $\begin{array}{l}\text { Night } \\
(\mathrm{mi})\end{array}$ \\
\hline 1660 & Nitric oxide & 100 & 0.1 & 0.5 & 200 & 0.4 & 1.7 \\
\hline 1660 & Nitric oxide, compressed & 100 & 0.1 & 0.5 & 200 & 0.4 & 1.7 \\
\hline 1670 & Perchloromethyl mercaptan & 100 & 0.1 & 0.2 & 200 & 0.4 & 0.8 \\
\hline 1680 & Potassium cyanide (when spilled in water) & 100 & 0.1 & 0.3 & 1,000 & 0.6 & 2.4 \\
\hline 1680 & Potassium cyanide, solid (when spilled in water) & 100 & 0.1 & 0.3 & 1,000 & 0.6 & 2.4 \\
\hline 1689 & Sodium cyanide (when spilled in water) & 200 & 0.1 & 0.4 & 1,300 & 0.8 & 3.0 \\
\hline 1689 & Sodium cyanide, solid (when spilled in water) & 200 & 0.1 & 0.4 & 1,300 & 0.8 & 3.0 \\
\hline 1694 & CA (when used as a weapon) & 100 & 0.1 & 0.3 & 500 & 1.0 & 2.6 \\
\hline 1695 & Chloroacetone, stabilized & 100 & 0.1 & 0.2 & 300 & 0.5 & 0.9 \\
\hline 1697 & $\mathrm{CN}$ (when used as a weapon) & 100 & 0.1 & 0.3 & 400 & 0.7 & 2.0 \\
\hline 1698 & Adamsite (when used as a weapon) & 200 & 0.2 & 0.7 & 600 & 1.4 & 3.2 \\
\hline 1698 & DM (when used as a weapon) & 200 & 0.2 & 0.7 & 600 & 1.4 & 3.2 \\
\hline 1699 & DA (when used as a weapon) & 200 & 0.2 & 0.7 & 600 & 1.4 & 3.2 \\
\hline 1703 & Tetraethyl dithiopyrophosphate and gases, in solution & 200 & 0.2 & 1.2 & 1,300 & 2.5 & 5.1 \\
\hline 1703 & Tetraethyl dithiopyrophosphate and gases, mixtures & 200 & 0.2 & 1.2 & 1,300 & 2.5 & 5.1 \\
\hline 1703 & $\begin{array}{l}\text { Tetraethyl dithiopyrophosphate and gases, mixtures, or in } \\
\text { solution }\left(\mathrm{LC}_{50} \text { more than } 200 \mathrm{ppm} \text { but not more than }\right. \\
5,000 \mathrm{ppm})\end{array}$ & 100 & 0.1 & 0.6 & 600 & 1.1 & 2.8 \\
\hline 1703 & $\begin{array}{l}\text { Tetraethyl dithiopyrophosphate and gases, mixtures, or in } \\
\left.\text { solution ( } \text { LC }_{50} \text { not more than } 200 \mathrm{ppm}\right)\end{array}$ & 200 & 0.2 & 1.2 & 1,300 & 2.5 & 5.1 \\
\hline 1705 & Tetraethyl pyrophosphate and compressed gas mixtures & 100 & 0.2 & 1.2 & 1,400 & 2.5 & 5.2 \\
\hline 1705 & $\begin{array}{l}\text { Tetraethyl pyrophosphate and compressed gas mixtures } \\
\text { (LC }{ }_{50} \text { more than } 200 \mathrm{ppm} \text { but not more than } 5,000 \mathrm{ppm} \text { ) }\end{array}$ & 100 & 0.1 & 0.6 & 500 & 1.0 & 2.8 \\
\hline 1705 & $\begin{array}{l}\text { Tetraethyl pyrophosphate and compressed gas mixtures } \\
\left(\mathrm{LC}_{50} \text { not more than } 200 \mathrm{ppm}\right)\end{array}$ & 100 & 0.2 & 1.2 & 1,400 & 2.5 & 5.2 \\
\hline 1716 & Acetyl bromide (when spilled in water) & 100 & 0.1 & 0.2 & 300 & 0.5 & 1.4 \\
\hline 1717 & Acetyl chloride (when spilled in water) & 100 & 0.1 & 0.3 & 400 & 0.7 & 2.2 \\
\hline 1722 & Allyl chlorocarbonate & 100 & 0.2 & 0.5 & 700 & 1.2 & 2.4 \\
\hline 1722 & Allyl chloroformate & 100 & 0.2 & 0.5 & 700 & 1.2 & 2.4 \\
\hline 1724 & Allyltrichlorosilane, stabilized (when spilled in water) & 100 & 0.1 & 0.5 & 600 & 1.2 & 3.4 \\
\hline 1725 & Aluminum bromide, anhydrous (when spilled in water) & 100 & 0.1 & 0.3 & 300 & 0.4 & 1.6 \\
\hline 1726 & Aluminum chloride, anhydrous (when spilled in water) & 100 & 0.1 & 0.5 & 400 & 0.7 & 2.8 \\
\hline 1728 & Amyltrichlorosilane (when spilled in water) & 100 & 0.1 & 0.1 & 200 & 0.3 & 1.2 \\
\hline 1732 & Antimony pentafluoride (when spilled in water) & 100 & 0.1 & 0.6 & 600 & 1.2 & 3.4 \\
\hline 1741 & Boron trichloride & 100 & 0.1 & 0.2 & 200 & 0.4 & 1.1 \\
\hline 1744 & Bromine & 200 & 0.3 & 1.1 & 1,100 & 2.1 & 4.6 \\
\hline 1744 & Bromine, solution & 200 & 0.3 & 1.1 & 1,100 & 2.1 & 4.6 \\
\hline 1745 & Bromine pentafluoride (when spilled on land) & 100 & 0.2 & 0.9 & 900 & 1.7 & 4.3 \\
\hline 1745 & Bromine pentafluoride (when spilled in water) & 100 & 0.1 & 0.6 & 800 & 1.4 & 4.1 \\
\hline 1746 & Bromine trifluoride (when spilled on land) & 100 & 0.1 & 0.4 & 600 & 1.1 & 3 \\
\hline
\end{tabular}


TABLE A.1 (Cont.)

\begin{tabular}{|c|c|c|c|c|c|c|c|}
\hline \multirow[b]{3}{*}{$\begin{array}{l}\text { UN ID } \\
\text { No. }\end{array}$} & \multirow[b]{3}{*}{ Name of Material } & \multicolumn{3}{|c|}{ Small Spills } & \multicolumn{3}{|c|}{ Large Spills } \\
\hline & & \multirow{2}{*}{$\begin{array}{c}\text { First } \\
\text { Isolate } \\
\text { in All } \\
\text { Direc- } \\
\text { tions (ft) }\end{array}$} & \multicolumn{2}{|c|}{$\begin{array}{c}\text { Then Protect } \\
\text { Persons } \\
\text { Downwind } \\
\text { during }\end{array}$} & \multirow{2}{*}{$\begin{array}{c}\text { First } \\
\text { Isolate } \\
\text { in All } \\
\text { Direc- } \\
\text { tions (ft) }\end{array}$} & \multicolumn{2}{|c|}{$\begin{array}{l}\text { Then Protect } \\
\text { Persons } \\
\text { Downwind } \\
\text { during }\end{array}$} \\
\hline & & & $\begin{array}{l}\text { Day } \\
(\mathrm{mi})\end{array}$ & $\begin{array}{c}\text { Night } \\
(\mathrm{mi})\end{array}$ & & $\begin{array}{l}\text { Day } \\
\text { (mi) }\end{array}$ & $\begin{array}{l}\text { Night } \\
\text { (mi) }\end{array}$ \\
\hline 1746 & Bromine trifluoride (when spilled in water) & 100 & 0.1 & 0.6 & 700 & 1.2 & 3.6 \\
\hline 1747 & Butyltrichlorosilane (when spilled in water) & 100 & 0.1 & 0.2 & 200 & 0.4 & 1.3 \\
\hline 1749 & Chlorine trifluoride & 200 & 0.3 & 1.3 & 1,000 & 1.8 & 5.1 \\
\hline 1752 & Chloroacetyl chloride (when spilled on land) & 100 & 0.2 & 0.4 & 500 & 0.9 & 1.6 \\
\hline $1752^{-}$ & Chloroacetyl chloride (when spilled in water) & 100 & 0.1 & 0.1 & 200 & 0.3 & 1.0 \\
\hline 1754 & Chlorosulfonic acid (when spilled on land) & 100 & 0.1 & 0.1 & 100 & 0.2 & 0.3 \\
\hline 1754 & Chlorosulfonic acid (when spilled in water) & 100 & 0.1 & 0.4 & 300 & 0.5 & 1.7 \\
\hline 1754 & $\begin{array}{l}\text { Chlorosulfonic acid and Sulfur trioxide mixture } \\
\text { (when spilled on land) }\end{array}$ & 100 & 0.1 & 0.1 & 100 & 0.2 & 0.3 \\
\hline 1754 & $\begin{array}{l}\text { Chlorosulfonic acid and Sulfur trioxide mixture } \\
\text { (when spilled in water) }\end{array}$ & 100 & 0.1 & 0.4 & 300 & 0.5 & 1.7 \\
\hline 1754 & Chlorosulphonic acid (when spilled on land) & 200 & 0.2 & 0.6 & 1,000 & 1.5 & 4.0 \\
\hline 1754 & Chlorosulphonic acid (when spilled in water) & 100 & 0.1 & 0.4 & 300 & 0.5 & 1.7 \\
\hline 1754 & $\begin{array}{l}\text { Chlorosulphonic acid and Sulphur trioxide mixture } \\
\text { (when spilled on land) }\end{array}$ & 200 & 0.2 & 0.6 & 1,000 & 1.5 & 4.0 \\
\hline 1754 & $\begin{array}{l}\text { Chlorosulphonic acid and Sulphur trioxide mixture } \\
\text { (when spilled in water) }\end{array}$ & 100 & 0.1 & 0.4 & 300 & 0.5 & 1.7 \\
\hline 1754 & $\begin{array}{l}\text { Sulfur trioxide and Chlorosulfonic acid mixture } \\
\text { (when spilled on land) }\end{array}$ & 200 & 0.2 & 0.6 & 1,000 & 1.5 & 4.0 \\
\hline 1754 & $\begin{array}{l}\text { Sulfur trioxide and Chlorosulfonic acid mixture } \\
\text { (when spilled in water) }\end{array}$ & 100 & 0.1 & 0.4 & 300 & 0.5 & 1.7 \\
\hline 1754 & $\begin{array}{l}\text { Sulphur trioxide and Chlorosulphonic acid mixture } \\
\text { (when spilled on land) }\end{array}$ & 200 & 0.2 & 0.6 & 1,000 & 1.5 & 4.0 \\
\hline 1754 & $\begin{array}{l}\text { Sulphur trioxide and Chlorosulphonic acid mixture } \\
\text { (when spilled in water) }\end{array}$ & 100 & 0.1 & 0.4 & 300 & 0.5 & 1.7 \\
\hline 1758 & Chromium oxychloride (when spilled in water) & 100 & 0.1 & 0.1 & 100 & 0.2 & 0.8 \\
\hline 1763 & Cyclohexyltrichlorosilane (when spilled in water) & 100 & 0.1 & 0.2 & 300 & 0.5 & 1.9 \\
\hline 1766 & Dichlorophenyltrichlorosilane (when spilled in water) & 100 & 0.1 & 0.6 & 700 & 1.3 & 3.6 \\
\hline 1767 & Diethlydichlorosilane (when spilled in water) & 100 & 0.1 & 0.1 & 200 & 0.3 & 0.8 \\
\hline 1769 & Diphenyldichlorosilane (when spilled in water) & 100 & 0.1 & 0.1 & 100 & 0.2 & 0.8 \\
\hline 1771 & Dodecyltrichlorosilane (when spilled in water) & 100 & 0.1 & 0.1 & 200 & 0.3 & 1.2 \\
\hline 1777 & Fluorosulfonic acid (when spilled in water) & 100 & 0.1 & 0.3 & 400 & 0.6 & 2.1 \\
\hline 1777 & Fluorosulphonic acid (when spilled in water) & 100 & 0.1 & 0.3 & 400 & 0.6 & 2.1 \\
\hline 1784 & Hexyltrichlorosilane (when spilled in water) & 100 & 0.1 & 0.3 & 400 & 0.7 & 2.4 \\
\hline 1799 & Nonyltrichlorosilane (when spilled in water) & 100 & 0.1 & 0.2 & 200 & 0.4 & 1.6 \\
\hline 1800 & Octadecyltrichlorosilane (when spilled in water) & 100 & 0.1 & 0.2 & 300 & 0.5 & 1.8 \\
\hline 1801 & Octyltrichlorosilane (when spilled in water) & 100 & 0.1 & 0.2 & 200 & 0.4 & 1.6 \\
\hline 1804 & Phenyltrichlorosilane (when spilled in water) & 100 & 0.1 & 0.6 & 800 & 1.4 & 4.0 \\
\hline 1806 & Phosphorus pentachloride (when spilled in water) & 100 & 0.1 & 0.3 & 300 & 0.5 & 1.9 \\
\hline 1809 & Phosphorus trichloride (when spilled on land) & 100 & 0.1 & 0.3 & 500 & 1.0 & 2.2 \\
\hline 1809 & Phosphorus trichloride (when spilled in water) & 100 & 0.1 & 0.4 & 600 & 1.0 & 3.0 \\
\hline
\end{tabular}


TABLE A.1 (Cont.)

\begin{tabular}{|c|c|c|c|c|c|c|c|}
\hline \multirow[b]{3}{*}{$\begin{array}{l}\text { UN ID } \\
\text { No. }\end{array}$} & \multirow[b]{3}{*}{ Name of Material } & \multicolumn{3}{|c|}{ Small Spills } & \multicolumn{3}{|c|}{ Large Spills } \\
\hline & & \multirow{2}{*}{$\begin{array}{c}\text { First } \\
\text { Isolate } \\
\text { in All } \\
\text { Direc- } \\
\text { tions (ft) }\end{array}$} & \multicolumn{2}{|c|}{$\begin{array}{l}\text { Then Protect } \\
\text { Persons } \\
\text { Downwind } \\
\text { during }\end{array}$} & \multirow{2}{*}{$\begin{array}{c}\text { First } \\
\text { Isolate } \\
\text { in All } \\
\text { Direc- } \\
\text { tions (ft) } \\
\end{array}$} & \multicolumn{2}{|c|}{$\begin{array}{l}\text { Then Protect } \\
\text { Persons } \\
\text { Downwind } \\
\text { during }\end{array}$} \\
\hline & & & $\begin{array}{l}\text { Day } \\
(\mathrm{mi})\end{array}$ & $\begin{array}{c}\text { Night } \\
(\mathrm{mi})\end{array}$ & & $\begin{array}{l}\text { Day } \\
(\mathrm{mi})\end{array}$ & $\begin{array}{l}\text { Night } \\
(\mathrm{mi})\end{array}$ \\
\hline 1810 & Phosphorus oxychloride (when spilled on land) & 100 & 0.2 & 0.3 & 400 & 0.7 & 1.4 \\
\hline 1810 & Phosphorus oxychloride (when spilled in water) & 100 & 0.1 & 0.6 & 800 & 1.5 & 3.9 \\
\hline 1816 & Propyltrichlorosilane (when spilled in water) & 100 & 0.1 & 0.3 & 400 & 0.8 & 2.6 \\
\hline 1818 & Silicon tetrachloride (when spilled in water) & 100 & 0.1 & 0.4 & 500 & 1.0 & 2.9 \\
\hline 1828 & Sulfur chlorides (when spilled on land) & 100 & 0.1 & 0.1 & 300 & 0.6 & 1.1 \\
\hline 1828 & Sulfur chlorides (when spilled in water) & 100 & 0.1 & 0.4 & 500 & 0.9 & 3.0 \\
\hline 1828 & Sulphur chlorides (when spilled on land) & 100 & 0.1 & 0.1 & 300 & 0.6 & 1.1 \\
\hline 1828 & Sulphur chlorides (when spilled in water) & 100 & 0.1 & 0.4 & 500 & 0.9 & 3.0 \\
\hline 1829 & Sulfur trioxide & 200 & 0.2 & 0.6 & 1,000 & 1.5 & 4.0 \\
\hline 1829 & Sulfur trioxide, inhibited & 200 & 0.2 & 0.6 & 1,000 & 1.5 & 4.0 \\
\hline 1829 & Sulfur trioxide, stabilized & 200 & 0.2 & 0.6 & 1,000 & 1.5 & 4.0 \\
\hline 1829 & Sulfur trioxide, uninhibited & 200 & 0.2 & 0.6 & 1,000 & 1.5 & 4.0 \\
\hline 1829 & Sulphur trioxide & 200 & 0.2 & 0.6 & 1,000 & 1.5 & 4.0 \\
\hline 1829 & Sulphur trioxide, inhibited & 200 & 0.2 & 0.6 & 1,000 & 1.5 & 4.0 \\
\hline 1829 & Sulphur trioxide, stabilized & 200 & 0.2 & 0.6 & 1,000 & 1.5 & 4.0 \\
\hline 1829 & Sulphur trioxide, uninhibited & 200 & 0.2 & 0.6 & 1,000 & 1.5 & 4.0 \\
\hline 1831 & Sulfuric acid, fuming & 200 & 0.2 & 0.6 & 1,000 & 1.5 & 4.0 \\
\hline 1831 & $\begin{array}{l}\text { Sulfuric acid, fuming, with not less than } 30 \% \text { free } \\
\text { Sulfur trioxide }\end{array}$ & 200 & 0.2 & 0.6 & 1,000 & 1.5 & 4.0 \\
\hline 1831 & Sulphuric acid, fuming & 200 & 0.2 & 0.6 & 1,000 & 1.5 & 4.0 \\
\hline 1831 & $\begin{array}{l}\text { Sulphuric acid, fuming, with not less than } 30 \% \text { free } \\
\text { Sulphur trioxide }\end{array}$ & 200 & 0.2 & 0.6 & 1,000 & 1.5 & 4.0 \\
\hline 1834 & Sulfuryl chloride (when spilled on land) & 100 & 0.1 & 0.1 & 100 & 0.2 & 0.5 \\
\hline 1834 & Sulfuryl chloride (when spilled in water) & 100 & 0.1 & 0.2 & 300 & 0.5 & 1.8 \\
\hline 1834 & Sulphuryl chloride (when spilled on land) & 100 & 0.1 & 0.1 & 100 & 0.2 & 0.5 \\
\hline 1834 & Sulphuryl chloride (when spilled in water) & 100 & 0.1 & 0.2 & 300 & 0.5 & 1.8 \\
\hline 1836 & Thionyl chloride (when spilled on land) & 100 & 0.2 & 0.5 & 300 & 0.6 & 1.4 \\
\hline 1836 & Thionyl chloride (when spilled in water) & 200 & 0.2 & 1.1 & 1,500 & 2.8 & 6.5 \\
\hline 1838 & Titanium tetrachloride (when spilled on land) & 100 & 0.1 & 0.1 & 200 & 0.3 & 0.5 \\
\hline 1838 & Titanium tetrachloride (when spilled in water) & 100 & 0.1 & 0.3 & 400 & 0.7 & 2.3 \\
\hline 1859 & Silicon tetrafluoride & 100 & 0.1 & 0.1 & 200 & 0.3 & 0.5 \\
\hline 1859 & Silicon tetrafluoride, compressed & 100 & 0.1 & 0.1 & 200 & 0.3 & 0.5 \\
\hline 1892 & $\mathrm{ED}$ (when used as a weapon) & 100 & 0.2 & 0.5 & 400 & 0.8 & 1.6 \\
\hline 1892 & Ethyldichloroarsine & 100 & 0.1 & 0.2 & 200 & 0.4 & 0.7 \\
\hline 1898 & Acetyl iodide (when spilled in water) & 100 & 0.1 & 0.2 & 200 & 0.4 & 1.1 \\
\hline 1911 & Diborane & 200 & 0.2 & 1.0 & 600 & 1.1 & 3.4 \\
\hline 1911 & Diborane, compressed & 200 & 0.2 & 1.0 & 600 & 1.1 & 3.4 \\
\hline 1923 & Calcium dithionite (when spilled in water) & 100 & 0.1 & 0.1 & 200 & 0.3 & 0.8 \\
\hline 1923 & Calcium hydrosulfite (when spilled in water) & 100 & 0.1 & 0.1 & 200 & 0.3 & 0.8 \\
\hline 1923 & Calcium hydrosulphite (when spilled in water) & 100 & 0.1 & 0.1 & 200 & 0.3 & 0.8 \\
\hline
\end{tabular}


TABLE A.1 (Cont.)

\begin{tabular}{|c|c|c|c|c|c|c|c|}
\hline \multirow[b]{3}{*}{$\begin{array}{l}\text { UN ID } \\
\text { No. }\end{array}$} & \multirow[b]{3}{*}{ Name of Material } & \multicolumn{3}{|c|}{ Small Spills } & \multicolumn{3}{|c|}{ Large Spills } \\
\hline & & \multirow{2}{*}{$\begin{array}{c}\text { First } \\
\text { Isolate } \\
\text { in All } \\
\text { Direc- } \\
\text { tions (ft) }\end{array}$} & \multicolumn{2}{|c|}{$\begin{array}{l}\text { Then Protect } \\
\text { Persons } \\
\text { Downwind } \\
\text { during }\end{array}$} & \multirow{2}{*}{$\begin{array}{c}\text { First } \\
\text { Isolate } \\
\text { in All } \\
\text { Direc- } \\
\text { tions (ft) }\end{array}$} & \multicolumn{2}{|c|}{$\begin{array}{c}\text { Then Protect } \\
\text { Persons } \\
\text { Downwind } \\
\text { during }\end{array}$} \\
\hline & & & $\begin{array}{l}\text { Day } \\
\text { (mi) }\end{array}$ & $\begin{array}{l}\text { Night } \\
\text { (mi) }\end{array}$ & & $\begin{array}{l}\text { Day } \\
\text { (mi) }\end{array}$ & $\begin{array}{l}\text { Night } \\
(\mathrm{mi})\end{array}$ \\
\hline 1931 & Zinc dithionite (when spilled in water) & 100 & 0.1 & 0.1 & 200 & 0.3 & 0.8 \\
\hline 1931 & Zinc hydrosulfite (when spilled in water) & 100 & 0.1 & 0.1 & 200 & 0.3 & 0.8 \\
\hline 1931 & Zinc hydrosulphite (when spilled in water) & 100 & 0.1 & 0.1 & 200 & 0.3 & 0.8 \\
\hline 1953 & $\begin{array}{l}\text { Compressed gas, flammable, poisonous, n.o.s. (Inhalation } \\
\text { Hazard Zone A) }\end{array}$ & 400 & 0.8 & 3.2 & 3,000 & 5.4 & 9.6 \\
\hline 1953 & $\begin{array}{l}\text { Compressed gas, flammable, poisonous, n.o.s. (Inhalation } \\
\text { Hazard Zone B) }\end{array}$ & 100 & 0.2 & 0.8 & 1,400 & 2.5 & 6.7 \\
\hline 1953 & $\begin{array}{l}\text { Compressed gas, flammable, poisonous, n.o.s. (Inhalation } \\
\text { Hazard Zone C) }\end{array}$ & 100 & 0.1 & 0.5 & 800 & 1.5 & 4.0 \\
\hline 1953 & $\begin{array}{l}\text { Compressed gas, flammable, poisonous, n.o.s. (Inhalation } \\
\text { Hazard Zone D) }\end{array}$ & 100 & 0.1 & 0.1 & 300 & 0.5 & 1.5 \\
\hline 1953 & $\begin{array}{l}\text { Compressed gas, flammable, toxic, n.o.s. (Inhalation Hazard } \\
\text { Zone A) }\end{array}$ & 400 & 0.8 & 3.2 & 3,000 & 5.4 & 9.6 \\
\hline 1953 & $\begin{array}{l}\text { Compressed gas, flammable, toxic, n.o.s. (Inhalation Hazard } \\
\text { Zone B) }\end{array}$ & 100 & 0.2 & 0.8 & 1,400 & 2.5 & 6.7 \\
\hline 1953 & $\begin{array}{l}\text { Compressed gas, flammable, toxic, n.o.s. (Inhalation Hazard } \\
\text { Zone C) }\end{array}$ & 100 & 0.1 & 0.5 & 800 & 1.5 & 4.0 \\
\hline 1953 & $\begin{array}{l}\text { Compressed gas, flammable, toxic, n.o.s. (Inhalation Hazard } \\
\text { Zone D) }\end{array}$ & 100 & 0.1 & 0.1 & 300 & 0.5 & 1.5 \\
\hline 1953 & Compressed gas, poisonous, flammable, n.o.s. & 400 & 0.8 & 3.2 & 3,000 & 5.4 & 9.6 \\
\hline 1953 & $\begin{array}{l}\text { Compressed gas, poisonous, flammable, n.o.s. } \\
\text { (Inhalation Hazard Zone A) }\end{array}$ & 400 & 0.8 & 3.2 & 3,000 & 5.4 & 9.6 \\
\hline 1953 & $\begin{array}{l}\text { Compressed gas, poisonous, flammable, n.o.s. } \\
\text { (Inhalation Hazard Zone B) }\end{array}$ & 100 & 0.2 & 0.8 & 1,400 & 2.5 & 6.7 \\
\hline 1953 & $\begin{array}{l}\text { Compressed gas, poisonous, flammable, n.o.s. } \\
\text { (Inhalation Hazard Zone C) }\end{array}$ & 100 & 0.1 & 0.5 & 800 & 1.5 & 4.0 \\
\hline 1953 & $\begin{array}{l}\text { Compressed gas, poisonous, flammable, n.o.s. } \\
\text { (Inhalation Hazard Zone D) }\end{array}$ & 100 & 0.1 & 0.1 & 300 & 0.5 & 1.5 \\
\hline 1953 & Compressed gas, toxic, flammable, n.o.s. & 400 & 0.8 & 3.2 & 3,000 & 5.4 & 9.6 \\
\hline 1953 & $\begin{array}{l}\text { Compressed gas, toxic, flammable, n.o.s. (Inhalation Hazard } \\
\text { Zone A) }\end{array}$ & 400 & 0.8 & 3.2 & 3,000 & 5.4 & 9.6 \\
\hline 1953 & $\begin{array}{l}\text { Compressed gas, toxic, flammable, n.o.s. (Inhalation Hazard } \\
\text { Zone B) }\end{array}$ & 100 & 0.2 & 0.8 & 1,400 & 2.5 & 6.7 \\
\hline 1953 & $\begin{array}{l}\text { Compressed gas, toxic, flammable, n.o.s. (Inhalation Hazard } \\
\text { Zone C) }\end{array}$ & 100 & 0.1 & 0.5 & 800 & 1.5 & 4.0 \\
\hline 1953 & $\begin{array}{l}\text { Compressed gas, toxic, flammable, n.o.s. (Inhalation Hazard } \\
\text { Zone D) }\end{array}$ & 100 & 0.1 & 0.1 & 300 & 0.5 & 1.5 \\
\hline 1953 & Liquefied gas, flammable, poisonous, n.o.s. & 400 & 0.8 & 3.2 & 3,000 & 5.4 & 9.6 \\
\hline 1953 & $\begin{array}{l}\text { Liquefied gas, flammable, poisonous, n.o.s. (Inhalation } \\
\text { Hazard Zone A) }\end{array}$ & 400 & 0.8 & 3.2 & 3,000 & 5.4 & 9.6 \\
\hline 1953 & $\begin{array}{l}\text { Liquefied gas, flammable, poisonous, n.o.s. (Inhalation } \\
\text { Hazard Zone B) }\end{array}$ & 100 & 0.2 & 0.8 & 1,400 & 2.5 & 6.7 \\
\hline
\end{tabular}


TABLE A.1 (Cont.)

\begin{tabular}{|c|c|c|c|c|c|c|c|}
\hline \multirow[b]{3}{*}{$\begin{array}{l}\text { UN ID } \\
\text { No. }\end{array}$} & \multirow[b]{3}{*}{ Name of Material } & \multicolumn{3}{|c|}{ Small Spills } & \multicolumn{3}{|c|}{ Large Spills } \\
\hline & & \multirow{2}{*}{$\begin{array}{c}\text { First } \\
\text { Isolate } \\
\text { in All } \\
\text { Direc- } \\
\text { tions (ft) }\end{array}$} & \multicolumn{2}{|c|}{$\begin{array}{l}\text { Then Protect } \\
\text { Persons } \\
\text { Downwind } \\
\text { during }\end{array}$} & \multirow{2}{*}{$\begin{array}{c}\text { First } \\
\text { Isolate } \\
\text { in All } \\
\text { Direc- } \\
\text { tions (ft) }\end{array}$} & \multicolumn{2}{|c|}{$\begin{array}{l}\text { Then Protect } \\
\text { Persons } \\
\text { Downwind } \\
\text { during }\end{array}$} \\
\hline & & & $\begin{array}{l}\text { Day } \\
(\mathrm{mi})\end{array}$ & $\begin{array}{l}\text { Night } \\
(\mathrm{mi})\end{array}$ & & $\begin{array}{l}\text { Day } \\
(\mathrm{mi})\end{array}$ & $\begin{array}{l}\text { Night } \\
(\mathrm{mi})\end{array}$ \\
\hline 1953 & $\begin{array}{l}\text { Liquefied gas, flammable, poisonous, n.o.s. (Inhalation } \\
\text { Hazard Zone C) }\end{array}$ & 100 & 0.1 & 0.5 & 800 & 1.5 & 4.0 \\
\hline 1953 & $\begin{array}{l}\text { Liquefied gas, flammable, poisonous, n.o.s. (Inhalation } \\
\text { Hazard Zone D) }\end{array}$ & 100 & 0.1 & 0.1 & 300 & 0.5 & 1.5 \\
\hline 1953 & Liquefied gas, flammable, toxic, n.o.s. & 400 & 0.8 & 3.2 & 3,000 & 5.4 & 9.6 \\
\hline 1953 & $\begin{array}{l}\text { Liquefied gas, flammable, toxic, n.o.s. (Inhalation Hazard } \\
\text { Zone A) }\end{array}$ & 400 & 0.8 & 3.2 & 3,000 & 5.4 & 9.6 \\
\hline 1953 & $\begin{array}{l}\text { Liquefied gas, flammable, toxic, n.o.s. (Inhalation Hazard } \\
\text { Zone B) }\end{array}$ & 100 & 0.2 & 0.8 & 1,400 & 2.5 & 6.7 \\
\hline 1953 & $\begin{array}{l}\text { Liquefied gas, flammable, toxic, n.o.s. (Inhalation Hazard } \\
\text { Zone C) }\end{array}$ & 100 & 0.1 & 0.5 & 800 & 1.5 & 4.0 \\
\hline 1953 & $\begin{array}{l}\text { Liquefied gas, flammable, toxic, n.o.s. (Inhalation Hazard } \\
\text { Zone D) }\end{array}$ & 100 & 0.1 & 0.1 & 300 & 0.5 & 1.5 \\
\hline 1955 & Compressed gas, poisonous, n.o.s. & 2,000 & 3.7 & 7.9 & 3,000 & 16.9 & 18.2 \\
\hline 1955 & $\begin{array}{l}\text { Compressed gas, poisonous, n.o.s. (Inhalation Hazard } \\
\text { Zone A) }\end{array}$ & 2,000 & 3.7 & 7.9 & 3,000 & 16.9 & 18.2 \\
\hline 1955 & $\begin{array}{l}\text { Compressed gas, poisonous, n.o.s. (Inhalation Hazard } \\
\text { Zone B) }\end{array}$ & 200 & 0.3 & 1.3 & 3,000 & 4.9 & 11.5 \\
\hline 1955 & $\begin{array}{l}\text { Compressed gas, poisonous, n.o.s. (Inhalation Hazard } \\
\text { Zone C) }\end{array}$ & 100 & 0.2 & 0.8 & 800 & 1.5 & 4.0 \\
\hline 1955 & $\begin{array}{l}\text { Compressed gas, poisonous, n.o.s. (Inhalation Hazard } \\
\text { Zone D) }\end{array}$ & 100 & 0.1 & 0.4 & 400 & 0.8 & 2.4 \\
\hline 1955 & Compressed gas, toxic, n.o.s. & 2,000 & 3.7 & 7.9 & 3,000 & 16.9 & 18.2 \\
\hline 1955 & Compressed gas, toxic, n.o.s. (Inhalation Hazard Zone A) & 2,000 & 3.7 & 7.9 & 3,000 & 16.9 & 18.2 \\
\hline 1955 & Compressed gas, toxic, n.o.s. (Inhalation Hazard Zone B) & 200 & 0.3 & 1.3 & 3,000 & 4.9 & 11.5 \\
\hline 1955 & Compressed gas, toxic, n.o.s. (Inhalation Hazard Zone C) & 100 & 0.2 & 0.8 & 800 & 1.5 & 4.0 \\
\hline 1955 & Compressed gas, toxic, n.o.s. (Inhalation Hazard Zone D) & 100 & 0.1 & 0.4 & 400 & 0.8 & 2.4 \\
\hline 1955 & Liquefied gas, poisonous, n.o.s. & 2,000 & 3.7 & 7.9 & 3,000 & 16.9 & 18.2 \\
\hline 1955 & Liquefied gas, poisonous, n.o.s. (Inhalation Hazard Zone A) & 2,000 & 3.7 & 7.9 & 3,000 & 16.9 & 18.2 \\
\hline 1955 & Liquefied gas, poisonous, n.o.s. (Inhalation Hazard Zone B) & 200 & 0.3 & 1.3 & 3,000 & 4.9 & 11.5 \\
\hline 1955 & Liquefied gas, poisonous, n.o.s. (Inhalation Hazard Zone C) & 100 & 0.2 & 0.8 & 800 & 1.5 & 4.0 \\
\hline 1955 & Liquefied gas, poisonous, n.o.s. (Inhalation Hazard Zone D) & 100 & 0.1 & 0.4 & 400 & 0.8 & 2.4 \\
\hline 1955 & Liquefied gas, toxic, n.o.s. & 2,000 & 3.7 & 7.9 & 3,000 & 16.9 & 18.2 \\
\hline 1955 & Liquefied gas, toxic, n.o.s. (Inhalation Hazard Zone A) & 2,000 & 3.7 & 7.9 & 3,000 & 16.9 & 18.2 \\
\hline 1955 & Liquefied gas, toxic, n.o.s. (Inhalation Hazard Zone B) & 200 & 0.3 & 1.3 & 3,000 & 4.9 & 11.5 \\
\hline 1955 & Liquefied gas, toxic, n.o.s. (Inhalation Hazard Zone C) & 100 & 0.2 & 0.8 & 800 & 1.5 & 4.0 \\
\hline 1955 & Liquefied gas, toxic, n.o.s. (Inhalation Hazard Zone D) & 100 & 0.1 & 0.4 & 400 & 0.8 & 2.4 \\
\hline 1955 & Organic phosphate compound mixed with compressed gas & 400 & 0.7 & 2.1 & 1,500 & 2.7 & 6.0 \\
\hline 1955 & Organic phosphate mixed with compressed gas & 400 & 0.7 & 2.1 & 1,500 & 2.7 & 6.0 \\
\hline 1955 & Organic phosphorus compound mixed with compressed gas & 400 & 0.7 & 2.1 & 1,500 & 2.7 & 6.0 \\
\hline 1967 & Insecticide gas, poisonous, n.o.s. & 400 & 0.7 & 2.1 & 1,500 & 2.7 & 6.0 \\
\hline 1967 & Insecticide gas, toxic, n.o.s. & 400 & 0.7 & 2.1 & 1,500 & 2.7 & 6.0 \\
\hline
\end{tabular}


TABLE A.1 (Cont.)

\begin{tabular}{|c|c|c|c|c|c|c|c|}
\hline \multirow[b]{3}{*}{$\begin{array}{l}\text { UN ID } \\
\text { No. }\end{array}$} & \multirow[b]{3}{*}{ Name of Material } & \multicolumn{3}{|c|}{ Small Spills } & \multicolumn{3}{|c|}{ Large Spills } \\
\hline & & \multirow{2}{*}{$\begin{array}{c}\text { First } \\
\text { Isolate } \\
\text { in All } \\
\text { Direc- } \\
\text { tions (ft) }\end{array}$} & \multicolumn{2}{|c|}{$\begin{array}{l}\text { Then Protect } \\
\text { Persons } \\
\text { Downwind } \\
\text { during }\end{array}$} & \multirow{2}{*}{$\begin{array}{c}\text { First } \\
\text { Isolate } \\
\text { in All } \\
\text { Direc- } \\
\text { tions (ft) } \\
\end{array}$} & \multicolumn{2}{|c|}{$\begin{array}{l}\text { Then Protect } \\
\text { Persons } \\
\text { Downwind } \\
\text { during }\end{array}$} \\
\hline & & & $\begin{array}{l}\text { Day } \\
(\mathrm{mi})\end{array}$ & $\begin{array}{c}\text { Night } \\
(\mathrm{mi})\end{array}$ & & $\begin{array}{l}\text { Day } \\
(\mathrm{mi})\end{array}$ & $\begin{array}{c}\text { Night } \\
\text { (mi) }\end{array}$ \\
\hline 1967 & Parathion and compressed gas mixture & 400 & 0.7 & 2.1 & 1,500 & 2.7 & 6.0 \\
\hline 1975 & Dinitrogen tetroxide and Nitric oxide mixture & 100 & 0.1 & 0.5 & 200 & 0.4 & 1.7 \\
\hline 1975 & Nitric oxide and Dinitrogen tetroxide mixture & 100 & 0.1 & 0.5 & 200 & 0.4 & 1.7 \\
\hline 1975 & Nitric oxide and Nitrogen dioxide mixture & 100 & 0.1 & 0.5 & 200 & 0.4 & 1.7 \\
\hline $1975^{\circ}$ & Nitric oxide and Nitrogen tetroxide mixture & 100 & 0.1 & 0.5 & 200 & 0.4 & 1.7 \\
\hline 1975 & Nitrogen dioxide and Nitric oxide mixture & 100 & 0.1 & 0.5 & 200 & 0.4 & 1.7 \\
\hline 1975 & Nitrogen tetroxide and Nitric oxide mixture & 100 & 0.1 & 0.5 & 200 & 0.4 & 1.7 \\
\hline 1994 & Iron pentacarbonyl & 100 & 0.2 & 0.4 & 500 & 1.0 & 1.9 \\
\hline 2004 & Magnesium diamide (when spilled in water) & 100 & 0.1 & 0.3 & 300 & 0.4 & 1.8 \\
\hline 2011 & Magnesium phosphide (when spilled in water) & 200 & 0.4 & 1.5 & 3,000 & 4.7 & 11.2 \\
\hline 2012 & Potassium phosphide (when spilled in water) & 200 & 0.3 & 1.1 & 1,600 & 2.9 & 8.6 \\
\hline 2013 & Strontium phosphide (when spilled in water) & 200 & 0.2 & 1.1 & 1,600 & 2.9 & 8.3 \\
\hline 2032 & Nitric acid, fuming & 100 & 0.1 & 0.2 & 200 & 0.4 & 0.8 \\
\hline 2032 & Nitric acid, red fuming & 100 & 0.1 & 0.2 & 200 & 0.4 & 0.8 \\
\hline 2186 & Hydrogen chloride, refrigerated liquid & 100 & 0.1 & 0.3 & 1,200 & 2.2 & 6.5 \\
\hline 2188 & Arsine & 200 & 0.4 & 1.9 & 1,400 & 2.6 & 5.9 \\
\hline 2188 & SA (when used as a weapon) & 200 & 0.5 & 1.5 & 1,300 & 2.5 & 5.0 \\
\hline 2189 & Dichlorosilane & 100 & 0.1 & 0.6 & 1,400 & 2.5 & 6.7 \\
\hline 2190 & Oxygen difluoride & 2,000 & 3.7 & 7.9 & 3,000 & 16.9 & 18.2 \\
\hline 2190 & Oxygen difluoride, compressed & 2,000 & 3.7 & 7.9 & 3,000 & 16.9 & 18.2 \\
\hline 2191 & Sulfuryl fluoride & 100 & 0.1 & 0.2 & 400 & 0.8 & 2.4 \\
\hline 2191 & Sulphuryl fluoride & 100 & 0.1 & 0.2 & 400 & 0.8 & 2.4 \\
\hline 2192 & Germane & 100 & 0.1 & 0.6 & 300 & 0.5 & 1.9 \\
\hline 2194 & Selenium hexafluoride & 300 & 0.5 & 2.0 & 1,500 & 2.7 & 5.6 \\
\hline 2195 & Tellurium hexafluoride & 300 & 0.6 & 2.5 & 2,000 & 3.7 & 7.1 \\
\hline 2196 & Tungsten hexafluoride & 100 & 0.1 & 0.7 & 400 & 0.6 & 2.3 \\
\hline 2197 & Hydrogen iodide, anhydrous & 100 & 0.1 & 0.2 & 400 & 0.8 & 2.3 \\
\hline 2198 & Phosphorus pentafluoride & 100 & 0.2 & 1.0 & 600 & 1.0 & 2.9 \\
\hline 2198 & Phosphorus pentafluoride, compressed & 100 & 0.2 & 1.0 & 600 & 1.0 & 2.9 \\
\hline 2199 & Phosphine & 200 & 0.4 & 1.9 & 1,400 & 2.7 & 6.0 \\
\hline 2202 & Hydrogen selenide, anhydrous & 400 & 0.8 & 3.2 & 3,000 & 5.4 & 9.6 \\
\hline 2204 & Carbonyl sulfide & 100 & 0.1 & 0.4 & 1,000 & 1.9 & 5.0 \\
\hline 2204 & Carbonyl sulphide & 100 & 0.1 & 0.4 & 1,000 & 1.9 & 5.0 \\
\hline 2232 & Chloroacetaldehyde & 100 & 0.1 & 0.2 & 300 & 0.5 & 1.0 \\
\hline 2232 & 2-Chloroethanal & 100 & 0.1 & 0.2 & 300 & 0.5 & 1.0 \\
\hline 2334 & Allylamine & 100 & 0.1 & 0.3 & 400 & 0.7 & 1.5 \\
\hline 2337 & Phenyl mercaptan & 100 & 0.1 & 0.1 & 200 & 0.2 & 0.4 \\
\hline 2382 & 1,2-Dimethylhydrazine & 100 & 0.1 & 0.1 & 200 & 0.4 & 0.8 \\
\hline 2382 & Dimethylhydrazine, symmetrical & 100 & 0.1 & 0.1 & 200 & 0.4 & 0.8 \\
\hline 2407 & Isopropyl chloroformate & 100 & 0.1 & 0.2 & 300 & 0.5 & 0.9 \\
\hline
\end{tabular}


TABLE A.1 (Cont.)

\begin{tabular}{|c|c|c|c|c|c|c|c|}
\hline \multirow[b]{3}{*}{$\begin{array}{l}\text { UN ID } \\
\text { No. }\end{array}$} & \multirow[b]{3}{*}{ Name of Material } & \multicolumn{3}{|c|}{ Small Spills } & \multicolumn{3}{|c|}{ Large Spills } \\
\hline & & \multirow{2}{*}{$\begin{array}{c}\text { First } \\
\text { Isolate } \\
\text { in All } \\
\text { Direc- } \\
\text { tions (ft) }\end{array}$} & \multicolumn{2}{|c|}{$\begin{array}{c}\text { Then Protect } \\
\text { Persons } \\
\text { Downwind } \\
\text { during }\end{array}$} & \multirow{2}{*}{\begin{tabular}{|c|} 
First \\
Isolate \\
in All \\
Direc- \\
tions (ft) \\
\end{tabular}} & \multicolumn{2}{|c|}{$\begin{array}{l}\text { Then Protect } \\
\text { Persons } \\
\text { Downwind } \\
\text { during }\end{array}$} \\
\hline & & & $\begin{array}{l}\text { Day } \\
(\mathrm{mi})\end{array}$ & $\begin{array}{c}\text { Night } \\
(\mathrm{mi})\end{array}$ & & $\begin{array}{l}\text { Day } \\
\text { (mi) }\end{array}$ & $\begin{array}{l}\text { Night } \\
\text { (mi) }\end{array}$ \\
\hline 2417 & Carbonyl fluoride & 100 & 0.1 & 0.7 & 300 & 0.6 & 2.3 \\
\hline 2417 & Carbonyl fluoride, compressed & 100 & 0.1 & 0.7 & 300 & 0.6 & 2.3 \\
\hline 2418 & Sulfur tetrafluoride & 200 & 0.4 & 2.0 & 1,600 & 2.9 & 6.6 \\
\hline 2418 & Sulphur tetrafluoride & 200 & 0.4 & 2.0 & 1,600 & 2.9 & 6.6 \\
\hline 2420 & Hexafluoroacetone & 100 & 0.2 & 0.8 & 2,500 & 4.5 & 10.1 \\
\hline 2421 & Nitrogen trioxide & 100 & 0.1 & 0.3 & 200 & 0.3 & 1.2 \\
\hline 2437 & Methylphenyldichlorosilane (when spilled in water) & 100 & 0.1 & 0.1 & 100 & 0.2 & 0.7 \\
\hline 2438 & Trimethylacetyl chloride & 100 & 0.1 & 0.1 & 200 & 0.3 & 0.5 \\
\hline 2442 & Trichloroacetyl chloride & 100 & 0.2 & 0.5 & 400 & 0.8 & 1.4 \\
\hline 2474 & Thiophosgene & 300 & 0.5 & 1.5 & 1,200 & 2.3 & 4.2 \\
\hline 2477 & Methyl isothiocyanate & 100 & 0.1 & 0.1 & 200 & 0.3 & 0.7 \\
\hline 2480 & Methyl isocyanate & 200 & 0.3 & 1.2 & 1,800 & 3.3 & 7.0 \\
\hline 2481 & Ethyl isocyanate & 200 & 0.4 & 1.3 & 2,500 & 3.9 & 7.5 \\
\hline 2482 & n-Propyl isocyanate & 400 & 0.7 & 1.6 & 3,000 & 5.6 & 8.6 \\
\hline 2483 & Isopropyl isocyanate & 400 & 0.7 & 1.8 & 3,000 & 7.4 & 9.8 \\
\hline 2484 & tert-Butyl isocyanate & 300 & 0.6 & 1.5 & 3,000 & 5.2 & 8.6 \\
\hline 2485 & n-Butyl isocyanate & 300 & 0.5 & 1.0 & 1,600 & 2.9 & 5.0 \\
\hline 2486 & Isobutyl isocyanate & 300 & $0.5^{-}$ & 1.0 & 1,600 & 3.0 & 4.8 \\
\hline 2487 & Phenyl isocyanate & 100 & 0.2 & 0.3 & 600 & 1.0 & 1.8 \\
\hline 2488 & Cyclohexyl isocyanate & 100 & 0.2 & 0.2 & 300 & 0.6 & 1.0 \\
\hline 2495 & Iodine pentafluoride (when spilled in water) & 100 & 0.1 & 0.6 & 700 & 1.2 & 3.6 \\
\hline 2521 & Diketene, inhibited & 100 & 0.1 & 0.1 & 100 & 0.2 & 0.3 \\
\hline 2521 & Diketene, stabilized & 100 & 0.1 & 0.1 & 100 & 0.2 & 0.3 \\
\hline 2534 & Methylchlorosilane & 100 & 0.1 & 0.5 & 800 & 1.5 & 4.0 \\
\hline 2548 & Chlorine pentafluoride & 100 & 0.2 & 1.1 & 800 & 1.5 & 4.6 \\
\hline 2600 & Carbon monoxide and Hydrogen mixture & 100 & 0.1 & 0.1 & 300 & 0.4 & 1.5 \\
\hline 2600 & Carbon monoxide and Hydrogen mixture, compressed & 100 & 0.1 & 0.1 & 300 & 0.4 & 1.5 \\
\hline 2600 & Hydrogen and Carbon monoxide mixture & 100 & 0.1 & 0.1 & 300 & 0.4 & 1.5 \\
\hline 2600 & Hydrogen and Carbon monoxide mixture, compressed & 100 & 0.1 & 0.1 & 300 & 0.4 & 1.5 \\
\hline 2605 & Methoxymethyl isocyanate & 200 & 0.2 & 0.4 & 600 & 1.0 & 1.6 \\
\hline 2606 & Methyl orthosilicate & 100 & 0.1 & 0.1 & 200 & 0.3 & 0.4 \\
\hline 2644 & Methyl iodide & 100 & 0.1 & 0.1 & 100 & 0.2 & 0.5 \\
\hline 2646 & Hexachlorocyclopentadiene & 100 & 0.1 & 0.1 & 200 & 0.3 & 0.3 \\
\hline 2668 & Chloroacetonitrile & 100 & 0.1 & 0.1 & 100 & 0.2 & 0.3 \\
\hline 2676 & Stibine & 200 & 0.3 & 1.4 & 900 & 1.7 & 4.7 \\
\hline 2691 & Phosphorus pentabromide (when spilled in water) & 100 & 0.1 & 0.4 & 300 & 0.4 & 1.7 \\
\hline 2692 & Boron tribromide (when spilled on land) & 100 & 0.1 & 0.3 & 200 & 0.4 & 0.8 \\
\hline 2692 & Boron tribromide (when spilled in water) & 100 & 0.1 & 0.3 & 300 & 0.5 & 1.6 \\
\hline 2740 & n-Propyl chloroformate & 100 & 0.1 & 0.2 & 300 & 0.5 & 0.9 \\
\hline 2742 & sec-Butyl chloroformate & 100 & 0.1 & 0.1 & 200 & 0.3 & 0.4 \\
\hline
\end{tabular}


TABLE A.1 (Cont.)

\begin{tabular}{|c|c|c|c|c|c|c|c|}
\hline \multirow[b]{3}{*}{$\begin{array}{l}\text { UN ID } \\
\text { No. }\end{array}$} & \multirow[b]{3}{*}{ Name of Material } & \multicolumn{3}{|c|}{ Small Spills } & \multicolumn{3}{|c|}{ Large Spills } \\
\hline & & \multirow{2}{*}{$\begin{array}{c}\text { First } \\
\text { Isolate } \\
\text { in All } \\
\text { Direc- } \\
\text { tions (ft) }\end{array}$} & \multicolumn{2}{|c|}{$\begin{array}{c}\text { Then Protect } \\
\text { Persons } \\
\text { Downwind } \\
\text { during }\end{array}$} & \multirow{2}{*}{$\begin{array}{c}\text { First } \\
\text { Isolate } \\
\text { in All } \\
\text { Direc- } \\
\text { tions (ft) }\end{array}$} & \multicolumn{2}{|c|}{$\begin{array}{l}\text { Then Protect } \\
\text { Persons } \\
\text { Downwind } \\
\text { during }\end{array}$} \\
\hline & & & $\begin{array}{l}\text { Day } \\
\text { (mi) }\end{array}$ & $\begin{array}{l}\text { Night } \\
\text { (mi) }\end{array}$ & & $\begin{array}{l}\text { Day } \\
\text { (mi) }\end{array}$ & $\begin{array}{l}\text { Night } \\
\text { (mi) }\end{array}$ \\
\hline 2742 & Isobutyl chloroformate & 100 & 0.1 & 0.1 & 100 & 0.2 & 0.3 \\
\hline 2743 & n-Butyl chloroformate & 100 & 0.1 & 0.1 & 100 & 0.2 & 0.3 \\
\hline 2806 & Lithium nitride (when spilled in water) & 100 & 0.1 & 0.2 & 200 & 0.4 & 1.6 \\
\hline 2810 & Buzz (when used as a weapon) & 100 & 0.1 & 0.3 & 200 & 0.3 & 1.2 \\
\hline 2810 & $\mathrm{BZ}$ (when used as a weapon) & 100 & 0.1 & 0.3 & 200 & 0.3 & 1.2 \\
\hline 2810 & CS (when used as a weapon) & 200 & 0.2 & 0.7 & 800 & 1.6 & 3.5 \\
\hline $2810^{-}$ & DC (when used as a weapon) & 100 & 0.1 & 0.5 & 800 & 1.4 & 3.3 \\
\hline 2810 & GA (when used as a weapon) & 100 & 0.2 & 0.4 & 500 & 1.0 & 1.9 \\
\hline 2810 & GB (when used as a weapon) & 500 & 1.0 & 2.1 & 3,000 & 7.1 & 7.1 \\
\hline 2810 & GD (when used as a weapon) & 300 & 0.5 & 1.1 & 2,500 & 4.2 & 6.5 \\
\hline 2810 & GF (when used as a weapon) & 100 & 0.2 & 0.4 & 800 & 1.4 & 3.2 \\
\hline $2810^{-}$ & $\mathrm{H}$ (when used as a weapon) & 100 & 0.1 & 0.1 & 200 & 0.4 & 0.7 \\
\hline $2810^{-}$ & $\mathrm{HD}$ (when used as a weapon) & 100 & 0.1 & 0.1 & 200 & 0.4 & 0.7 \\
\hline 2810 & HL (when used as a weapon) & 100 & 0.1 & 0.2 & 300 & 0.6 & 1.1 \\
\hline 2810 & HN-1 (when used as a weapon) & 100 & 0.1 & 0.1 & 200 & 0.4 & 0.8 \\
\hline 2810 & HN-2 (when used as a weapon) & 100 & 0.1 & 0.1 & 200 & 0.3 & 0.7 \\
\hline 2810 & HN-3 (when used as a weapon) & 100 & 0.1 & 0.1 & 100 & 0.1 & 0.2 \\
\hline 2810 & L (Lewisite) (when used as a weapon) & 100 & 0.1 & 0.2 & 300 & 0.6 & 1.1 \\
\hline 2810 & Lewisite (when used as a weapon) & 100 & 0.1 & 0.2 & 300 & 0.6 & 1.1 \\
\hline 2810 & Mustard (when used as a weapon) & 100 & 0.1 & 0.1 & 100 & 0.1 & 0.2 \\
\hline 2810 & Mustard lewisite (when used as a weapon) & 100 & 0.1 & 0.2 & 300 & 0.6 & 1.1 \\
\hline 2810 & Poisonous liquid, n.o.s. & 500 & 0.8 & 2.2 & 3,000 & 9.9 & 12.4 \\
\hline 2810 & Poisonous liquid, n.o.s. (Inhalation Hazard Zone A) & 500 & 0.8 & 2.2 & 3,000 & 9.9 & 12.4 \\
\hline 2810 & Poisonous liquid, n.o.s. (Inhalation Hazard Zone B) & 200 & 0.3 & 1.1 & 1,100 & 2.1 & 4.6 \\
\hline 2810 & Poisonous liquid, organic, n.o.s. & 500 & 0.8 & 2.1 & 3,000 & 9.9 & 12.4 \\
\hline 2810 & Poisonous liquid, organic, n.o.s. (Inhalation Hazard Zone A) & 500 & 0.8 & 2.1 & 3,000 & 9.9 & 12.4 \\
\hline 2810 & Poisonous liquid, organic, n.o.s. (Inhalation Hazard Zone B) & 200 & 0.2 & 0.6 & 900 & 1.6 & 3.5 \\
\hline 2810 & Sarin (when used as a weapon) & 500 & 1.0 & 2.1 & 3,000 & 7.1 & 7.1 \\
\hline 2810 & Soman (when used as a weapon) & 300 & 0.5 & 1.1 & 2,500 & 4.2 & 6.5 \\
\hline 2810 & Tabun (when used as a weapon) & 100 & 0.2 & 0.4 & 500 & 1.0 & 1.9 \\
\hline 2810 & Thickened GD (when used as a weapon) & 300 & 0.5 & 1.1 & 2,500 & 4.2 & 6.5 \\
\hline 2810 & Toxic liquid, n.o.s. & 500 & 0.8 & 2.2 & 3,000 & 9.9 & 12.4 \\
\hline 2810 & Toxic liquid, n.o.s. (Inhalation Hazard Zone A) & 500 & 0.8 & 2.2 & 3,000 & 9.9 & 12.4 \\
\hline 2810 & Toxic liquid, n.o.s. (Inhalation Hazard Zone B) & 200 & 0.3 & 1.1 & 1,100 & 2.1 & 4.6 \\
\hline 2810 & Toxic liquid, organic, n.o.s. & 500 & 0.8 & 2.1 & 3,000 & 9.9 & 12.4 \\
\hline 2810 & Toxic liquid, organic, n.o.s. (Inhalation Hazard Zone A) & 500 & 0.8 & 2.1 & 3,000 & 9.9 & 12.4 \\
\hline 2810 & Toxic liquid, organic, n.o.s. (Inhalation Hazard Zone B) & 200 & 0.2 & 0.6 & 900 & 1.6 & 3.5 \\
\hline 2810 & VX (when used as a weapon) & 100 & 0.1 & 0.1 & 200 & 0.4 & 0.6 \\
\hline 2811 & CX (when used as a weapon) & 100 & 0.1 & 0.3 & 300 & 0.6 & 1.9 \\
\hline 2826 & Ethyl chlorothioformate & 100 & 0.1 & 0.1 & 200 & 0.4 & 0.6 \\
\hline
\end{tabular}


TABLE A.1 (Cont.)

\begin{tabular}{|c|c|c|c|c|c|c|c|}
\hline \multirow[b]{3}{*}{$\begin{array}{l}\text { UN ID } \\
\text { No. }\end{array}$} & \multirow[b]{3}{*}{ Name of Material } & \multicolumn{3}{|c|}{ Small Spills } & \multicolumn{3}{|c|}{ Large Spills } \\
\hline & & \multirow{2}{*}{$\begin{array}{c}\text { First } \\
\text { Isolate } \\
\text { in All } \\
\text { Direc- } \\
\text { tions (ft) }\end{array}$} & \multicolumn{2}{|c|}{$\begin{array}{l}\text { Then Protect } \\
\text { Persons } \\
\text { Downwind } \\
\text { during }\end{array}$} & \multirow{2}{*}{$\begin{array}{c}\text { First } \\
\text { Isolate } \\
\text { in All } \\
\text { Direc- } \\
\text { tions (ft) }\end{array}$} & \multicolumn{2}{|c|}{$\begin{array}{l}\text { Then Protect } \\
\text { Persons } \\
\text { Downwind } \\
\text { during }\end{array}$} \\
\hline & & & $\begin{array}{l}\text { Day } \\
(\mathrm{mi})\end{array}$ & $\begin{array}{l}\text { Night } \\
\text { (mi) }\end{array}$ & & $\begin{array}{l}\text { Day } \\
(\mathrm{mi})\end{array}$ & $\begin{array}{l}\text { Night } \\
(\mathrm{mi})\end{array}$ \\
\hline 2845 & Ethyl phosphonous dichloride, anhydrous & 100 & 0.2 & 0.5 & 700 & 1.2 & 2.2 \\
\hline 2845 & Methyl phosphonous dichloride & 200 & 0.3 & 0.8 & 1,000 & 1.9 & 3.7 \\
\hline 2901 & Bromine chloride & 100 & 0.2 & 0.6 & 800 & 1.5 & 3.9 \\
\hline 2927 & Ethyl phosphonothioic dichloride, anhydrous & 100 & 0.1 & 0.1 & 100 & 0.1 & 0.2 \\
\hline 2927 & Ethyl phosphorodichloridate & 100 & 0.1 & 0.1 & 100 & 0.2 & 0.2 \\
\hline 2927 & Poisonous liquid, corrosive, n.o.s. & 300 & 0.5 & 1.5 & 2,500 & 3.9 & 7.5 \\
\hline 2927 & $\begin{array}{l}\text { Poisonous liquids, corrosive, n.o.s. (Inhalation Hazard } \\
\text { Zone A) }\end{array}$ & 300 & 0.5 & 1.5 & 2,500 & 3.9 & 7.5 \\
\hline 2927 & $\begin{array}{l}\text { Poisonous liquids, corrosive, n.o.s. (Inhalation Hazard } \\
\text { Zone B) }\end{array}$ & 200 & 0.3 & 1.1 & 1,100 & 2.1 & 4.6 \\
\hline 2927 & Toxic liquid, corrosive, organic, n.o.s. & 200 & 0.4 & 1.3 & 2,500 & 3.9 & 7.5 \\
\hline 2927 & $\begin{array}{l}\text { Toxic liquid, corrosive, organic, n.o.s. (Inhalation Hazard } \\
\text { Zone A) }\end{array}$ & 200 & 0.4 & 1.3 & 2,500 & 3.9 & 7.5 \\
\hline 2927 & $\begin{array}{l}\text { Toxic liquid, corrosive, organic, n.o.s. (Inhalation Hazard } \\
\text { Zone B) }\end{array}$ & 100 & 0.2 & 0.4 & 600 & 1.0 & 1.8 \\
\hline 2929 & Poisonous liquid, flammable, n.o.s. & 500 & 0.8 & 2.2 & 3,000 & 9.9 & 12.4 \\
\hline 2929 & $\begin{array}{l}\text { Poisonous liquid, flammable, n.o.s. (Inhalation Hazard } \\
\text { Zone A) }\end{array}$ & 500 & 0.8 & 2.2 & 3,000 & 9.9 & 12.4 \\
\hline 2929 & $\begin{array}{l}\text { Poisonous liquid, flammable, n.o.s. (Inhalation Hazard } \\
\text { Zone B) }\end{array}$ & 200 & 0.2 & 0.6 & 900 & 1.6 & 3.5 \\
\hline 2929 & Poisonous liquid, flammable, organic, n.o.s. & 500 & 0.8 & 2.1 & 3,000 & 9.9 & 12.4 \\
\hline 2929 & $\begin{array}{l}\text { Poisonous liquid, flammable, organic, n.o.s. (Inhalation } \\
\text { Hazard Zone A) }\end{array}$ & 500 & 0.8 & 2.1 & 3,000 & 9.9 & 12.4 \\
\hline 2929 & $\begin{array}{l}\text { Poisonous liquid, flammable, organic, n.o.s. (Inhalation } \\
\text { Hazard Zone B) }\end{array}$ & 200 & 0.2 & 0.6 & 900 & 1.6 & 3.5 \\
\hline 2929 & Toxic liquid, flammable, n.o.s. & 500 & 0.8 & 2.2 & 3,000 & 9.9 & 12.4 \\
\hline 2929 & Toxic liquid, flammable, n.o.s. (Inhalation Hazard Zone A) & 500 & 0.8 & 2.2 & 3,000 & 9.9 & 12.4 \\
\hline 2929 & Toxic liquid, flammable, n.o.s. (Inhalation Hazard Zone B) & 200 & 0.2 & 0.6 & 900 & 1.6 & 3.5 \\
\hline 2929 & Toxic liquid, flammable, organic, n.o.s. & 500 & 0.8 & 2.1 & 3,000 & 9.9 & 12.4 \\
\hline 2929 & $\begin{array}{l}\text { Toxic liquid, flammable, organic, n.o.s. (Inhalation Hazard } \\
\text { Zone A) }\end{array}$ & 500 & 0.8 & 2.1 & 3,000 & 9.9 & 12.4 \\
\hline 2929 & $\begin{array}{l}\text { Toxic liquid, flammable, organic, n.o.s. (Inhalation Hazard } \\
\text { Zone B) }\end{array}$ & 200 & 0.2 & 0.6 & 900 & 1.6 & 3.5 \\
\hline 2977 & $\begin{array}{l}\text { Radioactive material, Uranium hexafluoride, fissile (when } \\
\text { spilled in water) }\end{array}$ & 100 & 0.1 & 0.4 & 300 & 0.5 & 2.1 \\
\hline 2977 & $\begin{array}{l}\text { Uranium hexafluoride, fissile containing more than } 1 \% \\
\text { Uranium-235 (when spilled in water) }\end{array}$ & 100 & 0.1 & 0.4 & 300 & 0.5 & 2.1 \\
\hline 2978 & $\begin{array}{l}\text { Radioactive material, Uranium hexafluoride (when spilled in } \\
\text { water) }\end{array}$ & 100 & 0.1 & 0.4 & 300 & 0.5 & 2.1 \\
\hline 2978 & $\begin{array}{l}\text { Radioactive material, Uranium hexafluoride, non fissile or } \\
\text { fissile-excepted (when spilled in water) }\end{array}$ & 100 & 0.1 & 0.4 & 300 & 0.5 & 2.1 \\
\hline 2978 & Uranium hexafluoride (when spilled in water) & 100 & 0.1 & 0.4 & 300 & 0.5 & 2.1 \\
\hline
\end{tabular}


TABLE A.1 (Cont.)

\begin{tabular}{|c|c|c|c|c|c|c|c|}
\hline \multirow[b]{3}{*}{$\begin{array}{l}\text { UN ID } \\
\text { No. }\end{array}$} & \multirow[b]{3}{*}{ Name of Material } & \multicolumn{3}{|c|}{ Small Spills } & \multicolumn{3}{|c|}{ Large Spills } \\
\hline & & \multirow{2}{*}{$\begin{array}{c}\text { First } \\
\text { Isolate } \\
\text { in All } \\
\text { Direc- } \\
\text { tions (ft) }\end{array}$} & \multicolumn{2}{|c|}{$\begin{array}{l}\text { Then Protect } \\
\text { Persons } \\
\text { Downwind } \\
\text { during }\end{array}$} & \multirow{2}{*}{$\begin{array}{c}\text { First } \\
\text { Isolate } \\
\text { in All } \\
\text { Direc- } \\
\text { tions (ft) }\end{array}$} & \multicolumn{2}{|c|}{$\begin{array}{l}\text { Then Protect } \\
\text { Persons } \\
\text { Downwind } \\
\text { during }\end{array}$} \\
\hline & & & $\begin{array}{l}\text { Day } \\
\text { (mi) }\end{array}$ & $\begin{array}{l}\text { Night } \\
\text { (mi) }\end{array}$ & & $\begin{array}{l}\text { Day } \\
\text { (mi) }\end{array}$ & $\begin{array}{l}\text { Night } \\
\text { (mi) }\end{array}$ \\
\hline 2978 & $\begin{array}{l}\text { Uranium hexafluoride, fissile-excepted (when spilled in } \\
\text { water) }\end{array}$ & 100 & 0.1 & 0.4 & 300 & 0.5 & 2.1 \\
\hline 2978 & $\begin{array}{l}\text { Uranium hexafluoride, low specific activity (when spilled in } \\
\text { water) }\end{array}$ & 100 & 0.1 & 0.4 & 300 & 0.5 & 2.1 \\
\hline 2978 & Uranium hexafluoride, non-fissile (when spilled in water) & 100 & 0.1 & 0.4 & 300 & 0.5 & 2.1 \\
\hline 2985 & $\begin{array}{l}\text { Chlorosilanes, flammable, corrosive, n.o.s. (when spilled in } \\
\text { water) }\end{array}$ & 100 & 0.1 & 0.3 & 500 & 0.8 & 2.4 \\
\hline 2985 & Chlorosilanes, n.o.s.(when spilled in water) & 100 & 0.1 & 0.3 & 500 & 0.8 & 2.4 \\
\hline 2986 & $\begin{array}{l}\text { Chlorosilanes, corrosive, flammable, n.o.s. (when spilled in } \\
\text { water) }\end{array}$ & 100 & 0.1 & 0.3 & 500 & 0.8 & 2.4 \\
\hline 2986 & Chlorosilanes, n.o.s. (when spilled in water) & 100 & 0.1 & 0.3 & 500 & 0.8 & 2.4 \\
\hline 2987 & Chlorosilanes, corrosive, n.o.s. (when spilled in water) & 100 & 0.1 & 0.3 & 500 & 0.8 & 2.4 \\
\hline 2987 & Chlorosilanes, n.o.s. (when spilled in water) & 100 & 0.1 & 0.3 & 500 & 0.8 & 2.4 \\
\hline 2988 & Chlorosilanes, n.o.s. (when spilled in water) & 100 & 0.1 & 0.3 & 500 & 0.8 & 2.4 \\
\hline 2988 & $\begin{array}{l}\text { Chlorosilanes, water-reactive, flammable, corrosive, n.o.s. } \\
\text { (when spilled in water) }\end{array}$ & 100 & 0.1 & 0.3 & 500 & 0.8 & 2.4 \\
\hline 3023 & 2-Methyl-2-hepthanethiol & 100 & 0.1 & 0.1 & 200 & 0.3 & 0.5 \\
\hline 3023 & tert-Octyl mercaptan & 100 & 0.1 & 0.1 & 200 & 0.3 & 0.5 \\
\hline 3048 & Aluminum phosphide pesticide (when spilled in water) & 300 & 0.4 & 1.7 & 3,000 & 5.6 & 12.2 \\
\hline 3049 & Metal alkyl halides, n.o.s. (when spilled in water) & 100 & 0.1 & 0.1 & 100 & 0.2 & 0.8 \\
\hline 3049 & $\begin{array}{l}\text { Metal alkyl halides, water-reactive, n.o.s. (when spilled in } \\
\text { water) }\end{array}$ & 100 & 0.1 & 0.1 & 100 & 0.2 & 0.8 \\
\hline 3049 & Metal aryl halides, n.o.s. (when spilled in water) & 100 & 0.1 & 0.1 & 100 & 0.2 & 0.8 \\
\hline 3049 & $\begin{array}{l}\text { Metal aryl halides, water-reactive, n.o.s. (when spilled in } \\
\text { water) }\end{array}$ & 100 & 0.1 & 0.1 & 100 & 0.2 & 0.8 \\
\hline 3052 & Aluminum alkyl halides (when spilled in water) & 100 & 0.1 & 0.1 & 100 & 0.2 & 0.8 \\
\hline 3052 & Aluminum alkyl halides, liquid (when spilled in water) & 100 & 0.1 & 0.1 & 100 & 0.2 & 0.8 \\
\hline 3052 & Aluminum alkyl halides, solid (when spilled in water) & 100 & 0.1 & 0.1 & 100 & 0.2 & 0.8 \\
\hline 3057 & Trifluoroacetyl chloride & 100 & 0.2 & 0.8 & 3,000 & 4.9 & 11.5 \\
\hline 3079 & Methacrylonitrile, inhibited & 100 & 0.1 & 0.2 & 300 & 0.5 & 1.0 \\
\hline 3079 & Methacrylonitrile, stabilized & 100 & 0.1 & 0.2 & 300 & 0.5 & 1.0 \\
\hline 3083 & Perchloryl fluoride & 100 & 0.1 & 0.4 & 1,200 & 2.2 & 5.5 \\
\hline 3122 & Poisonous liquid, oxidizing, n.o.s. & 500 & 0.8 & 2.2 & 3,000 & 9.9 & 12.4 \\
\hline 3122 & $\begin{array}{l}\text { Poisonous liquids, oxidizing, n.o.s. (Inhalation Hazard } \\
\text { Zone A) }\end{array}$ & 500 & 0.8 & 2.2 & 3,000 & 9.9 & 12.4 \\
\hline 3122 & $\begin{array}{l}\text { Poisonous liquids, oxidizing, n.o.s. (Inhalation Hazard } \\
\text { Zone B) }\end{array}$ & 100 & 0.2 & 0.9 & 900 & 1.7 & 4.3 \\
\hline 3122 & Toxic liquid, oxidizing, n.o.s. & 500 & 0.8 & 2.2 & 3,000 & 9.9 & 12.4 \\
\hline 3122 & Toxic liquid, oxidizing, n.o.s. (Inhalation Hazard Zone A) & 500 & 0.8 & 2.2 & 3,000 & 9.9 & 12.4 \\
\hline 3122 & Toxic liquid, oxidizing, n.o.s. (Inhalation Hazard Zone B) & 100 & 0.2 & 0.9 & 900 & 1.7 & 4.3 \\
\hline 3123 & Poisonous liquid, water-reactive, n.o.s. & 500 & 0.8 & 2.2 & 3,000 & 9.9 & 12.4 \\
\hline
\end{tabular}


TABLE A.1 (Cont.)

\begin{tabular}{|c|c|c|c|c|c|c|c|}
\hline \multirow[b]{3}{*}{$\begin{array}{l}\text { UN ID } \\
\text { No. }\end{array}$} & \multirow[b]{3}{*}{ Name of Material } & \multicolumn{3}{|c|}{ Small Spills } & \multicolumn{3}{|c|}{ Large Spills } \\
\hline & & \multirow{2}{*}{$\begin{array}{c}\text { First } \\
\text { Isolate } \\
\text { in All } \\
\text { Direc- } \\
\text { tions (ft) }\end{array}$} & \multicolumn{2}{|c|}{$\begin{array}{l}\text { Then Protect } \\
\text { Persons } \\
\text { Downwind } \\
\text { during }\end{array}$} & \multirow{2}{*}{$\begin{array}{c}\text { First } \\
\text { Isolate } \\
\text { in All } \\
\text { Direc- } \\
\text { tions (ft) }\end{array}$} & \multicolumn{2}{|c|}{$\begin{array}{l}\text { Then Protect } \\
\text { Persons } \\
\text { Downwind } \\
\text { during }\end{array}$} \\
\hline & & & $\begin{array}{l}\text { Day } \\
(\mathrm{mi})\end{array}$ & $\begin{array}{l}\text { Night } \\
\text { (mi) }\end{array}$ & & $\begin{array}{l}\text { Day } \\
(\mathrm{mi})\end{array}$ & $\begin{array}{c}\text { Night } \\
(\mathrm{mi})\end{array}$ \\
\hline 3123 & $\begin{array}{l}\text { Poisonous liquid, water-reactive, n.o.s. (Inhalation Hazard } \\
\text { Zone A) }\end{array}$ & 500 & 0.8 & 2.2 & 3,000 & 9.9 & 12.4 \\
\hline 3123 & $\begin{array}{l}\text { Poisonous liquid, water-reactive, n.o.s. (Inhalation Hazard } \\
\text { Zone B) }\end{array}$ & 200 & 0.3 & 1.1 & 1,100 & 2.1 & 4.6 \\
\hline 3123 & $\begin{array}{l}\text { Poisonous liquid, which in contact with water emits } \\
\text { flammable gases, n.o.s. }\end{array}$ & 500 & 0.8 & 2.2 & 3,000 & 9.9 & 12.4 \\
\hline 3123 & $\begin{array}{l}\text { Poisonous liquid, which in contact with water emit } \\
\text { flammable gases, n.o.s. (Inhalation Hazard Zone A) }\end{array}$ & 500 & 0.8 & 2.2 & 3,000 & 9.9 & 12.4 \\
\hline 3123 & $\begin{array}{l}\text { Poisonous liquid, which in contact with water emit } \\
\text { flammable gases, n.o.s. (Inhalation Hazard Zone B) }\end{array}$ & 200 & 0.3 & 1.1 & 1,100 & 2.1 & 4.6 \\
\hline 3123 & Toxic liquid, water-reactive, n.o.s. & 500 & 0.8 & 2.2 & 3,000 & 9.9 & 12.4 \\
\hline 3123 & $\begin{array}{l}\text { Toxic liquid, water-reactive, n.o.s. (Inhalation Hazard } \\
\text { Zone A) }\end{array}$ & 500 & 0.8 & 2.2 & 3,000 & 9.9 & 12.4 \\
\hline 3123 & $\begin{array}{l}\text { Toxic liquid, water-reactive, n.o.s. (Inhalation Hazard } \\
\text { Zone B) }\end{array}$ & 200 & 0.3 & 1.1 & 1,100 & 2.1 & 4.6 \\
\hline 3123 & $\begin{array}{l}\text { Toxic liquid, which in contact with water emits flammable } \\
\text { gases, n.o.s. }\end{array}$ & 500 & 0.8 & 2.2 & 3,000 & 9.9 & 12.4 \\
\hline 3123 & $\begin{array}{l}\text { Toxic liquids, which in contact with water emit flammable } \\
\text { gases, n.o.s. (Inhalation Hazard Zone A) }\end{array}$ & 500 & 0.8 & 2.2 & 3,000 & 9.9 & 12.4 \\
\hline 3123 & $\begin{array}{l}\text { Toxic liquids, which in contact with water emit flammable } \\
\text { gases, n.o.s. (Inhalation Hazard Zone B) }\end{array}$ & 200 & 0.3 & 1.1 & 1,100 & 2.1 & 4.6 \\
\hline 3160 & Liquefied gas, poisonous, flammable, n.o.s. & 400 & 0.8 & 3.2 & 3,000 & 5.4 & 9.6 \\
\hline 3160 & $\begin{array}{l}\text { Liquefied gas, poisonous, flammable, n.o.s. (Inhalation } \\
\text { Hazard Zone A) }\end{array}$ & 400 & 0.8 & 3.2 & 3,000 & 5.4 & 9.6 \\
\hline 3160 & $\begin{array}{l}\text { Liquefied gas, poisonous, flammable, n.o.s. (Inhalation } \\
\text { Hazard Zone B) }\end{array}$ & 100 & 0.2 & 0.8 & 1,400 & 2.5 & 6.7 \\
\hline 3160 & $\begin{array}{l}\text { Liquefied gas, poisonous, flammable, n.o.s. (Inhalation } \\
\text { Hazard Zone C) }\end{array}$ & 100 & 0.1 & 0.5 & 800 & 1.5 & 4.0 \\
\hline 3160 & $\begin{array}{l}\text { Liquefied gas, poisonous, flammable, n.o.s. (Inhalation } \\
\text { Hazard Zone D) }\end{array}$ & 100 & 0.1 & 0.1 & 300 & 0.5 & 1.5 \\
\hline 3160 & Liquefied gas, toxic, flammable, n.o.s. & 400 & 0.8 & 3.2 & 3,000 & 5.4 & 9.6 \\
\hline 3160 & $\begin{array}{l}\text { Liquefied gas, toxic, flammable, n.o.s. (Inhalation Hazard } \\
\text { Zone A) }\end{array}$ & 400 & 0.8 & 3.2 & 3,000 & 5.4 & 9.6 \\
\hline 3160 & $\begin{array}{l}\text { Liquefied gas, toxic, flammable, n.o.s. (Inhalation Hazard } \\
\text { Zone B) }\end{array}$ & 100 & 0.2 & 0.8 & 1,400 & 2.5 & 6.7 \\
\hline 3160 & $\begin{array}{l}\text { Liquefied gas, toxic, flammable, n.o.s. (Inhalation Hazard } \\
\text { Zone C) }\end{array}$ & 100 & 0.1 & 0.5 & 800 & 1.5 & 4.0 \\
\hline 3160 & $\begin{array}{l}\text { Liquefied gas, toxic, flammable, n.o.s. (Inhalation Hazard } \\
\text { Zone D) }\end{array}$ & 100 & 0.1 & 0.1 & 300 & 0.5 & 1.5 \\
\hline 3162 & Liquefied gas, poisonous, n.o.s. & 2,000 & 3.7 & 7.9 & 3,000 & 16.9 & 18.2 \\
\hline 3162 & Liquefied gas, poisonous, n.o.s. (Inhalation Hazard Zone A) & 2,000 & 3.7 & 7.9 & 3,000 & 16.9 & 18.2 \\
\hline 3162 & Liquefied gas, poisonous, n.o.s. (Inhalation Hazard Zone B) & 200 & 0.3 & 1.3 & 3,000 & 4.9 & 11.5 \\
\hline 3162 & Liquefied gas, poisonous, n.o.s. (Inhalation Hazard Zone C) & 100 & 0.2 & 0.8 & 800 & 1.5 & 4.0 \\
\hline
\end{tabular}


TABLE A.1 (Cont.)

\begin{tabular}{|c|c|c|c|c|c|c|c|}
\hline \multirow[b]{3}{*}{$\begin{array}{l}\text { UN ID } \\
\text { No. }\end{array}$} & \multirow[b]{3}{*}{ Name of Material } & \multicolumn{3}{|c|}{ Small Spills } & \multicolumn{3}{|c|}{ Large Spills } \\
\hline & & \multirow{2}{*}{$\begin{array}{c}\text { First } \\
\text { Isolate } \\
\text { in All } \\
\text { Direc- } \\
\text { tions (ft) }\end{array}$} & \multicolumn{2}{|c|}{$\begin{array}{l}\text { Then Protect } \\
\text { Persons } \\
\text { Downwind } \\
\text { during }\end{array}$} & \multirow{2}{*}{$\begin{array}{c}\text { First } \\
\text { Isolate } \\
\text { in All } \\
\text { Direc- } \\
\text { tions (ft) }\end{array}$} & \multicolumn{2}{|c|}{$\begin{array}{l}\text { Then Protect } \\
\text { Persons } \\
\text { Downwind } \\
\text { during }\end{array}$} \\
\hline & & & $\begin{array}{l}\text { Day } \\
(\mathrm{mi})\end{array}$ & $\begin{array}{l}\text { Night } \\
(\mathrm{mi})\end{array}$ & & $\begin{array}{l}\text { Day } \\
(\mathrm{mi})\end{array}$ & $\begin{array}{c}\text { Night } \\
(\mathrm{mi})\end{array}$ \\
\hline 3162 & Liquefied gas, poisonous, n.o.s. (Inhalation Hazard Zone D) & 100 & 0.1 & 0.4 & 400 & 0.8 & 2.4 \\
\hline 3162 & Liquefied gas, toxic, n.o.s. & 2,000 & 3.7 & 7.9 & 3,000 & 16.9 & 18.2 \\
\hline 3162 & Liquefied gas, toxic, n.o.s. (Inhalation Hazard Zone A) & 2,000 & 3.7 & 7.9 & 3,000 & 16.9 & 18.2 \\
\hline 3162 & Liquefied gas, toxic, n.o.s. (Inhalation Hazard Zone B) & 200 & 0.3 & 1.3 & 3,000 & 4.9 & 11.5 \\
\hline 3162 & Liquefied gas, toxic, n.o.s. (Inhalation Hazard Zone C) & 100 & 0.2 & 0.8 & 800 & 1.5 & 4.0 \\
\hline 3162 & Liquefied gas, toxic, n.o.s. (Inhalation Hazard Zone D) & 100 & 0.1 & 0.4 & 400 & 0.8 & 2.4 \\
\hline 3246 & Methanesulfonyl chloride & 200 & 0.2 & 0.4 & 500 & 1.0 & 1.6 \\
\hline 3246 & Methanesulphonyl chloride & 200 & 0.2 & 0.4 & 500 & 1.0 & 1.6 \\
\hline 3275 & Nitriles, poisonous, flammable, n.o.s. & 100 & 0.1 & 0.2 & 300 & 0.5 & 1.0 \\
\hline 3275 & Nitriles, toxic, flammable, n.o.s. & 100 & 0.1 & 0.2 & 300 & 0.5 & 1.0 \\
\hline 3276 & Nitriles, poisonous, liquid n.o.s. & 100 & 0.1 & 0.2 & 300 & 0.5 & 1.0 \\
\hline 3276 & Nitriles, poisonous, n.o.s. & 100 & 0.1 & 0.2 & 300 & 0.5 & 1.0 \\
\hline 3276 & Nitriles, toxic, liquid, n.o.s. & 100 & 0.1 & 0.2 & 300 & 0.5 & 1.0 \\
\hline 3276 & Nitriles, toxic, n.o.s. & 100 & 0.1 & 0.2 & 300 & 0.5 & 1.0 \\
\hline 3278 & Organophosphorus compound, poisonous, liquid, n.o.s. & 200 & 0.3 & 0.8 & 1,000 & 1.9 & 3.7 \\
\hline 3278 & Organophosphorus compound, poisonous, n.o.s. & 200 & 0.3 & 0.8 & 1,000 & 1.9 & 3.7 \\
\hline 3278 & Organophosphorus compound, toxic, liquid, n.o.s. & 200 & 0.3 & 0.8 & 1,000 & 1.9 & 3.7 \\
\hline 3278 & Organophosphorus compound, toxic, n.o.s. & 200 & 0.3 & 0.8 & 1,000 & 1.9 & 3.7 \\
\hline 3279 & Organophosphorus compound, poisonous, flammable, n.o.s. & 200 & 0.3 & 0.8 & 1,000 & 1.9 & 3.7 \\
\hline 3279 & Organophosphorus compound, toxic, flammable, n.o.s. & 200 & 0.3 & 0.8 & 1,000 & 1.9 & 3.7 \\
\hline 3280 & Organoarsenic compound, liquid, n.o.s. & 100 & 0.1 & 0.4 & 700 & 1.3 & 3.2 \\
\hline 3280 & Organoarsenic compound, n.o.s. & 100 & 0.1 & 0.4 & 700 & 1.3 & 3.2 \\
\hline 3281 & Metal carbonyls, liquid, n.o.s. & 300 & 0.5 & 2.2 & 1,600 & 2.9 & 6.1 \\
\hline 3281 & Metal carbonyls, n.o.s. & 300 & 0.5 & 2.2 & 1,600 & 2.9 & 6.1 \\
\hline 3287 & Poisonous liquid, inorganic, n.o.s. & 300 & 0.6 & 2.2 & 1,800 & 3.3 & 6.9 \\
\hline 3287 & $\begin{array}{l}\text { Poisonous liquid, inorganic, n.o.s. (Inhalation Hazard } \\
\text { Zone A) }\end{array}$ & 300 & 0.6 & 2.2 & 1,800 & 3.3 & 6.9 \\
\hline 3287 & $\begin{array}{l}\text { Poisonous liquid, inorganic, n.o.s. (Inhalation Hazard } \\
\text { Zone B) }\end{array}$ & 200 & 0.3 & 1.1 & 1,100 & 2.1 & 4.6 \\
\hline 3287 & Toxic liquid, inorganic, n.o.s. & 300 & 0.6 & 2.2 & 1,800 & 3.3 & 6.9 \\
\hline 3287 & Toxic liquid, inorganic, n.o.s. (Inhalation Hazard Zone A) & 300 & 0.6 & 2.2 & 1,800 & 3.3 & 6.9 \\
\hline 3287 & Toxic liquid, inorganic, n.o.s. (Inhalation Hazard Zone B) & 200 & 0.3 & 1.1 & 1,100 & 2.1 & 4.6 \\
\hline 3289 & Poisonous liquid, corrosive, inorganic, n.o.s. & 300 & 0.6 & 2.2 & 1,800 & 3.3 & 6.9 \\
\hline 3289 & $\begin{array}{l}\text { Poisonous liquid, corrosive, inorganic, n.o.s. (Inhalation } \\
\text { Hazard Zone A) }\end{array}$ & 300 & 0.6 & 2.2 & 1,800 & 3.3 & 6.9 \\
\hline 3289 & $\begin{array}{l}\text { Poisonous liquid, corrosive, inorganic, n.o.s. (Inhalation } \\
\text { Hazard Zone B) }\end{array}$ & 200 & 0.3 & 1.1 & 1,100 & 2.1 & 4.6 \\
\hline 3289 & Toxic liquid, corrosive, inorganic, n.o.s. & 300 & 0.6 & 2.2 & 1,800 & 3.3 & 6.9 \\
\hline 3289 & $\begin{array}{l}\text { Toxic liquid, corrosive, inorganic, n.o.s. (Inhalation Hazard } \\
\text { Zone A) }\end{array}$ & 300 & 0.6 & 2.2 & 1,800 & 3.3 & 6.9 \\
\hline
\end{tabular}


TABLE A.1 (Cont.)

\begin{tabular}{|c|c|c|c|c|c|c|c|}
\hline \multirow[b]{3}{*}{$\begin{array}{l}\text { UN ID } \\
\text { No. }\end{array}$} & \multirow[b]{3}{*}{ Name of Material } & \multicolumn{3}{|c|}{ Small Spills } & \multicolumn{3}{|c|}{ Large Spills } \\
\hline & & \multirow{2}{*}{$\begin{array}{c}\text { First } \\
\text { Isolate } \\
\text { in All } \\
\text { Direc- } \\
\text { tions (ft) }\end{array}$} & \multicolumn{2}{|c|}{$\begin{array}{l}\text { Then Protect } \\
\text { Persons } \\
\text { Downwind } \\
\text { during }\end{array}$} & \multirow{2}{*}{$\begin{array}{c}\text { First } \\
\text { Isolate } \\
\text { in All } \\
\text { Direc- } \\
\text { tions (ft) }\end{array}$} & \multicolumn{2}{|c|}{$\begin{array}{l}\text { Then Protect } \\
\text { Persons } \\
\text { Downwind } \\
\text { during }\end{array}$} \\
\hline & & & $\begin{array}{l}\text { Day } \\
(\mathrm{mi})\end{array}$ & $\begin{array}{l}\text { Night } \\
\text { (mi) }\end{array}$ & & $\begin{array}{l}\text { Day } \\
(\mathrm{mi})\end{array}$ & $\begin{array}{l}\text { Night } \\
(\mathrm{mi})\end{array}$ \\
\hline 3289 & $\begin{array}{l}\text { Toxic liquid, corrosive, inorganic, n.o.s. (Inhalation Hazard } \\
\text { Zone B) }\end{array}$ & 200 & 0.3 & 1.1 & 1,100 & 2.1 & 4.6 \\
\hline 3294 & $\begin{array}{l}\text { Hydrogen cyanide, solution in alcohol, with not more than } \\
45 \% \text { Hydrogen cyanide }\end{array}$ & 100 & 0.1 & 0.2 & 700 & 0.4 & 1.3 \\
\hline 3300 & $\begin{array}{l}\text { Carbon dioxide and Ethylene oxide mixture, with more than } \\
87 \% \text { Ethylene oxide }\end{array}$ & 100 & 0.1 & 0.1 & 300 & 0.5 & 1.5 \\
\hline 3300 & $\begin{array}{l}\text { Ethylene oxide and Carbon dioxide mixture, with more than } \\
87 \% \text { Ethylene oxide }\end{array}$ & 100 & 0.1 & 0.1 & 300 & 0.5 & 1.5 \\
\hline 3303 & Compressed gas, poisonous, oxidizing, n.o.s. & 2,000 & 3.7 & 7.9 & 3,000 & 16.9 & 18.2 \\
\hline 3303 & $\begin{array}{l}\text { Compressed gas, poisonous, oxidizing, n.o.s. (Inhalation } \\
\text { Hazard Zone A) }\end{array}$ & 2,000 & 3.7 & 7.9 & 3,000 & 16.9 & 18.2 \\
\hline 3303 & $\begin{array}{l}\text { Compressed gas, poisonous, oxidizing, n.o.s. (Inhalation } \\
\text { Hazard Zone B) }\end{array}$ & 200 & 0.3 & 1.3 & 1,200 & 2.2 & 5.5 \\
\hline 3303 & $\begin{array}{l}\text { Compressed gas, poisonous, oxidizing, n.o.s. (Inhalation } \\
\text { Hazard Zone C) }\end{array}$ & 100 & 0.2 & 0.8 & 800 & 1.5 & 4.0 \\
\hline 3303 & $\begin{array}{l}\text { Compressed gas, poisonous, oxidizing, n.o.s. (Inhalation } \\
\text { Hazard Zone D) }\end{array}$ & 100 & 0.1 & 0.4 & 400 & 0.8 & 2.4 \\
\hline 3303 & Compressed gas, toxic, oxidizing, n.o.s. & 2,000 & 3.7 & 7.9 & 3,000 & 16.9 & 18.2 \\
\hline 3303 & $\begin{array}{l}\text { Compressed gas, toxic, oxidizing, n.o.s. (Inhalation Hazard } \\
\text { Zone A) }\end{array}$ & 2,000 & 3.7 & 7.9 & 3,000 & 16.9 & 18.2 \\
\hline 3303 & $\begin{array}{l}\text { Compressed gas, toxic, oxidizing, n.o.s. (Inhalation Hazard } \\
\text { Zone B) }\end{array}$ & 200 & 0.3 & 1.3 & 1,200 & 2.2 & 5.5 \\
\hline 3303 & $\begin{array}{l}\text { Compressed gas, toxic, oxidizing, n.o.s. (Inhalation Hazard } \\
\text { Zone C) }\end{array}$ & 100 & 0.2 & 0.8 & 800 & 1.5 & 4.0 \\
\hline 3303 & $\begin{array}{l}\text { Compressed gas, toxic, oxidizing, n.o.s. (Inhalation Hazard } \\
\text { Zone D) }\end{array}$ & 100 & 0.1 & 0.4 & 400 & 0.8 & 2.4 \\
\hline 3304 & Compressed gas, poisonous, corrosive, n.o.s. & 2,000 & 3.7 & 7.9 & 3,000 & 16.9 & 18.2 \\
\hline 3304 & $\begin{array}{l}\text { Compressed gas, poisonous, corrosive, n.o.s. (Inhalation } \\
\text { Hazard Zone A) }\end{array}$ & 2,000 & 3.7 & 7.9 & 3,000 & 16.9 & 18.2 \\
\hline 3304 & $\begin{array}{l}\text { Compressed gas, poisonous, corrosive, n.o.s. (Inhalation } \\
\text { Hazard Zone B) }\end{array}$ & 200 & 0.3 & 1.3 & 2,500 & 4.5 & 10.1 \\
\hline 3304 & $\begin{array}{l}\text { Compressed gas, poisonous, corrosive, n.o.s. (Inhalation } \\
\text { Hazard Zone C) }\end{array}$ & 100 & 0.2 & 0.8 & 800 & 1.5 & 4.0 \\
\hline 3304 & $\begin{array}{l}\text { Compressed gas, poisonous, corrosive, n.o.s. (Inhalation } \\
\text { Hazard Zone D) }\end{array}$ & 100 & 0.1 & 0.4 & 200 & 0.4 & 1.4 \\
\hline 3304 & Compressed gas, toxic, corrosive, n.o.s. & 2,000 & 3.7 & 7.9 & 3,000 & 16.9 & 18.2 \\
\hline 3304 & $\begin{array}{l}\text { Compressed gas, toxic, corrosive, n.o.s. (Inhalation Hazard } \\
\text { Zone A) }\end{array}$ & 2,000 & 3.7 & 7.9 & 3,000 & 16.9 & 18.2 \\
\hline 3304 & $\begin{array}{l}\text { Compressed gas, toxic, corrosive, n.o.s. (Inhalation Hazard } \\
\text { Zone B) }\end{array}$ & 200 & 0.3 & 1.3 & 2,500 & 4.5 & 10.1 \\
\hline 3304 & $\begin{array}{l}\text { Compressed gas, toxic, corrosive, n.o.s. (Inhalation Hazard } \\
\text { Zone C) }\end{array}$ & 100 & 0.2 & 0.8 & 800 & 1.5 & 4.0 \\
\hline 3304 & $\begin{array}{l}\text { Compressed gas, toxic, corrosive, n.o.s. (Inhalation Hazard } \\
\text { Zone D) }\end{array}$ & 100 & 0.1 & 0.4 & 200 & 0.4 & 1.4 \\
\hline
\end{tabular}


TABLE A.1 (Cont.)

\begin{tabular}{|c|c|c|c|c|c|c|c|}
\hline \multirow[b]{3}{*}{$\begin{array}{l}\text { UN ID } \\
\text { No. }\end{array}$} & \multirow[b]{3}{*}{ Name of Material } & \multicolumn{3}{|c|}{ Small Spills } & \multicolumn{3}{|c|}{ Large Spills } \\
\hline & & \multirow{2}{*}{$\begin{array}{c}\text { First } \\
\text { Isolate } \\
\text { in All } \\
\text { Direc- } \\
\text { tions (ft) }\end{array}$} & \multicolumn{2}{|c|}{$\begin{array}{l}\text { Then Protect } \\
\text { Persons } \\
\text { Downwind } \\
\text { during }\end{array}$} & \multirow{2}{*}{$\begin{array}{c}\text { First } \\
\text { Isolate } \\
\text { in All } \\
\text { Direc- } \\
\text { tions (ft) }\end{array}$} & \multicolumn{2}{|c|}{$\begin{array}{l}\text { Then Protect } \\
\text { Persons } \\
\text { Downwind } \\
\text { during }\end{array}$} \\
\hline & & & $\begin{array}{l}\text { Day } \\
(\mathrm{mi})\end{array}$ & $\begin{array}{c}\text { Night } \\
\text { (mi) }\end{array}$ & & $\begin{array}{l}\text { Day } \\
(\mathrm{mi})\end{array}$ & $\begin{array}{l}\text { Night } \\
(\mathrm{mi})\end{array}$ \\
\hline 3305 & Compressed gas, poisonous, flammable, corrosive, n.o.s. & 2,000 & 3.7 & 7.9 & 3,000 & 16.9 & 18.2 \\
\hline 3305 & $\begin{array}{l}\text { Compressed gas, poisonous, flammable, corrosive, n.o.s. } \\
\text { (Inhalation Hazard Zone A) }\end{array}$ & 2,000 & 3.7 & 7.9 & 3,000 & 16.9 & 18.2 \\
\hline 3305 & $\begin{array}{l}\text { Compressed gas, poisonous, flammable, corrosive, n.o.s. } \\
\text { (Inhalation Hazard Zone B) }\end{array}$ & 100 & 0.1 & 0.6 & 1,400 & 2.5 & 6.7 \\
\hline 3305 & $\begin{array}{l}\text { Compressed gas, poisonous, flammable, corrosive, n.o.s. } \\
\text { (Inhalation Hazard Zone C) }\end{array}$ & 100 & 0.1 & 0.5 & 800 & 1.5 & 4.0 \\
\hline 3305 & $\begin{array}{l}\text { Compressed gas, poisonous, flammable, corrosive, n.o.s. } \\
\text { (Inhalation Hazard Zone D) }\end{array}$ & 100 & 0.1 & 0.1 & 300 & 0.5 & 1.5 \\
\hline 3305 & Compressed gas, toxic, flammable, corrosive, n.o.s. & 2,000 & 3.7 & 7.9 & 3,000 & 16.9 & 18.2 \\
\hline 3305 & $\begin{array}{l}\text { Compressed gas, toxic, flammable, corrosive, n.o.s. } \\
\text { (Inhalation Hazard Zone A) }\end{array}$ & 2,000 & 3.7 & 7.9 & 3,000 & 16.9 & 18.2 \\
\hline 3305 & $\begin{array}{l}\text { Compressed gas, toxic, flammable, corrosive, n.o.s. } \\
\text { (Inhalation Hazard Zone B) }\end{array}$ & 100 & 0.1 & 0.6 & 1,400 & 2.5 & 6.7 \\
\hline 3305 & $\begin{array}{l}\text { Compressed gas, toxic, flammable, corrosive, n.o.s. } \\
\text { (Inhalation Hazard Zone C) }\end{array}$ & 100 & 0.1 & 0.5 & 800 & 1.5 & 4.0 \\
\hline 3305 & $\begin{array}{l}\text { Compressed gas, toxic, flammable, corrosive, n.o.s. } \\
\text { (Inhalation Hazard Zone D) }\end{array}$ & 100 & 0.1 & 0.1 & 300 & 0.5 & 1.5 \\
\hline 3306 & Compressed gas, poisonous, oxidizing, corrosive, n.o.s. & 2,000 & 3.7 & 7.9 & 3,000 & 16.9 & 18.2 \\
\hline 3306 & $\begin{array}{l}\text { Compressed gas, poisonous, oxidizing, corrosive, n.o.s. } \\
\text { (Inhalation Hazard Zone A) }\end{array}$ & 2,000 & 3.7 & 7.9 & 3,000 & 16.9 & 18.2 \\
\hline 3306 & $\begin{array}{l}\text { Compressed gas, poisonous, oxidizing, corrosive, n.o.s. } \\
\text { (Inhalation Hazard Zone B) }\end{array}$ & 200 & 0.3 & 1.3 & 1,200 & 2.2 & 5.5 \\
\hline 3306 & $\begin{array}{l}\text { Compressed gas, poisonous, oxidizing, corrosive, n.o.s. } \\
\text { (Inhalation Hazard Zone C) }\end{array}$ & 100 & 0.2 & 0.8 & 800 & 1.5 & 4.0 \\
\hline 3306 & $\begin{array}{l}\text { Compressed gas, poisonous, oxidizing, corrosive, n.o.s. } \\
\text { (Inhalation Hazard Zone D) }\end{array}$ & 100 & 0.1 & 0.4 & 200 & 0.4 & 1.4 \\
\hline 3306 & Compressed gas, toxic, oxidizing, corrosive, n.o.s. & 2,000 & 3.7 & 7.9 & 3,000 & 16.9 & 18.2 \\
\hline 3306 & $\begin{array}{l}\text { Compressed gas, toxic, oxidizing, corrosive, n.o.s. } \\
\text { (Inhalation Hazard Zone A) }\end{array}$ & 2,000 & 3.7 & 7.9 & 3,000 & 16.9 & 18.2 \\
\hline 3306 & $\begin{array}{l}\text { Compressed gas, toxic, oxidizing, corrosive, n.o.s. } \\
\text { (Inhalation Hazard Zone B) }\end{array}$ & 200 & 0.3 & 1.3 & 1,200 & 2.2 & 5.5 \\
\hline 3306 & $\begin{array}{l}\text { Compressed gas, toxic, oxidizing, corrosive, n.o.s. } \\
\text { (Inhalation Hazard Zone C) }\end{array}$ & 100 & 0.2 & 0.8 & 800 & 1.5 & 4.0 \\
\hline 3306 & $\begin{array}{l}\text { Compressed gas, toxic, oxidizing, corrosive, n.o.s. } \\
\text { (Inhalation Hazard Zone D) }\end{array}$ & 100 & 0.1 & 0.4 & 200 & 0.4 & 1.4 \\
\hline 3307 & Liquefied gas, poisonous, oxidizing, n.o.s. & 2,000 & 3.7 & 7.9 & 3,000 & 16.9 & 18.2 \\
\hline 3307 & $\begin{array}{l}\text { Liquefied gas, poisonous, oxidizing, n.o.s. (Inhalation } \\
\text { Hazard Zone A) }\end{array}$ & 2,000 & 3.7 & 7.9 & 3,000 & 16.9 & 18.2 \\
\hline 3307 & $\begin{array}{l}\text { Liquefied gas, poisonous, oxidizing, n.o.s. (Inhalation } \\
\text { Hazard Zone B) }\end{array}$ & 200 & 0.3 & 1.3 & 1,200 & 2.2 & 5.5 \\
\hline 3307 & $\begin{array}{l}\text { Liquefied gas, poisonous, oxidizing, n.o.s. (Inhalation } \\
\text { Hazard Zone C) }\end{array}$ & 100 & 0.2 & 0.8 & 800 & 1.5 & 4.0 \\
\hline
\end{tabular}


TABLE A.1 (Cont.)

\begin{tabular}{|c|c|c|c|c|c|c|c|}
\hline \multirow[b]{3}{*}{$\begin{array}{l}\text { UN ID } \\
\text { No. }\end{array}$} & \multirow[b]{3}{*}{ Name of Material } & \multicolumn{3}{|c|}{ Small Spills } & \multicolumn{3}{|c|}{ Large Spills } \\
\hline & & \multirow{2}{*}{$\begin{array}{c}\text { First } \\
\text { Isolate } \\
\text { in All } \\
\text { Direc- } \\
\text { tions (ft) }\end{array}$} & \multicolumn{2}{|c|}{$\begin{array}{l}\text { Then Protect } \\
\text { Persons } \\
\text { Downwind } \\
\text { during }\end{array}$} & \multirow{2}{*}{$\begin{array}{c}\text { First } \\
\text { Isolate } \\
\text { in All } \\
\text { Direc- } \\
\text { tions (ft) }\end{array}$} & \multicolumn{2}{|c|}{$\begin{array}{l}\text { Then Protect } \\
\text { Persons } \\
\text { Downwind } \\
\text { during }\end{array}$} \\
\hline & & & $\begin{array}{l}\text { Day } \\
(\mathrm{mi})\end{array}$ & $\begin{array}{l}\text { Night } \\
\text { (mi) }\end{array}$ & & $\begin{array}{l}\text { Day } \\
(\mathrm{mi})\end{array}$ & $\begin{array}{l}\text { Night } \\
(\mathrm{mi})\end{array}$ \\
\hline 3307 & $\begin{array}{l}\text { Liquefied gas, poisonous, oxidizing, n.o.s. (Inhalation } \\
\text { Hazard Zone D) }\end{array}$ & 100 & 0.1 & 0.4 & 400 & 0.8 & 2.4 \\
\hline 3307 & Liquefied gas, toxic, oxidizing, n.o.s. & 2,000 & 3.7 & 7.9 & 3,000 & 16.9 & 18.2 \\
\hline 3307 & $\begin{array}{l}\text { Liquefied gas, toxic, oxidizing, n.o.s. (Inhalation Hazard } \\
\text { Zone A) }\end{array}$ & 2,000 & 3.7 & 7.9 & 3,000 & 16.9 & 18.2 \\
\hline 3307 & $\begin{array}{l}\text { Liquefied gas, toxic, oxidizing, n.o.s. (Inhalation Hazard } \\
\text { Zone B) }\end{array}$ & 200 & 0.3 & 1.3 & 1,200 & 2.2 & 5.5 \\
\hline 3307 & $\begin{array}{l}\text { Liquefied gas, toxic, oxidizing, n.o.s. (Inhalation Hazard } \\
\text { Zone C) }\end{array}$ & 100 & 0.2 & 0.8 & 800 & 1.5 & 4.0 \\
\hline 3307 & $\begin{array}{l}\text { Liquefied gas, toxic, oxidizing, n.o.s. (Inhalation Hazard } \\
\text { Zone D) }\end{array}$ & 100 & 0.1 & 0.4 & 400 & 0.8 & 2.4 \\
\hline 3308 & Liquefied gas, poisonous, corrosive, n.o.s. & 2,000 & 3.7 & 7.9 & 3,000 & 16.9 & 18.2 \\
\hline 3308 & $\begin{array}{l}\text { Liquefied gas, poisonous, corrosive, n.o.s. (Inhalation Hazard } \\
\text { Zone A) }\end{array}$ & 2,000 & 3.7 & 7.9 & 3,000 & 16.9 & 18.2 \\
\hline 3308 & $\begin{array}{l}\text { Liquefied gas, poisonous, corrosive, n.o.s. (Inhalation Hazard } \\
\text { Zone B) }\end{array}$ & 200 & 0.3 & 1.3 & 2,500 & 4.5 & 10.1 \\
\hline 3308 & $\begin{array}{l}\text { Liquefied gas, poisonous, corrosive, n.o.s. (Inhalation Hazard } \\
\text { Zone C) }\end{array}$ & 100 & 0.2 & 0.8 & 800 & 1.5 & 4.0 \\
\hline 3308 & $\begin{array}{l}\text { Liquefied gas, poisonous, corrosive, n.o.s. (Inhalation Hazard } \\
\text { Zone D) }\end{array}$ & 100 & 0.1 & 0.4 & 200 & 0.4 & 1.4 \\
\hline 3308 & Liquefied gas, toxic, corrosive, n.o.s. & 2,000 & 3.7 & 7.9 & 3,000 & 16.9 & 18.2 \\
\hline 3308 & $\begin{array}{l}\text { Liquefied gas, toxic, corrosive, n.o.s. (Inhalation Hazard } \\
\text { Zone A) }\end{array}$ & 2,000 & 3.7 & 7.9 & 3,000 & 16.9 & 18.2 \\
\hline 3308 & $\begin{array}{l}\text { Liquefied gas, toxic, corrosive, n.o.s. (Inhalation Hazard } \\
\text { Zone B) }\end{array}$ & 200 & 0.3 & 1.3 & 2,500 & 4.5 & 10.1 \\
\hline 3308 & $\begin{array}{l}\text { Liquefied gas, toxic, corrosive, n.o.s. (Inhalation Hazard } \\
\text { Zone C) }\end{array}$ & 100 & 0.2 & 0.8 & 800 & 1.5 & 4.0 \\
\hline 3308 & $\begin{array}{l}\text { Liquefied gas, toxic, corrosive, n.o.s. (Inhalation Hazard } \\
\text { Zone D) }\end{array}$ & 100 & 0.1 & 0.4 & 200 & 0.4 & 1.4 \\
\hline 3309 & Liquefied gas, poisonous, flammable, corrosive, n.o.s & 2,000 & 3.7 & 7.9 & 3,000 & 16.9 & 18.2 \\
\hline 3309 & $\begin{array}{l}\text { Liquefied gas, poisonous, flammable, corrosive, n.o.s. } \\
\text { (Inhalation Hazard Zone A) }\end{array}$ & 2,000 & 3.7 & 7.9 & 3,000 & 16.9 & 18.2 \\
\hline 3309 & $\begin{array}{l}\text { Liquefied gas, poisonous, flammable, corrosive, n.o.s. } \\
\text { (Inhalation Hazard Zone B) }\end{array}$ & 100 & 0.1 & 0.6 & 1,400 & 2.5 & 6.7 \\
\hline 3309 & $\begin{array}{l}\text { Liquefied gas, poisonous, flammable, corrosive, n.o.s. } \\
\text { (Inhalation Hazard Zone C) }\end{array}$ & 100 & 0.1 & 0.5 & 800 & 1.5 & 4.0 \\
\hline 3309 & $\begin{array}{l}\text { Liquefied gas, poisonous, flammable, corrosive, n.o.s. } \\
\text { (Inhalation Hazard Zone D) }\end{array}$ & 100 & 0.1 & 0.1 & 300 & 0.5 & 1.5 \\
\hline 3309 & Liquefied gas, toxic, flammable, corrosive, n.o.s. & 2,000 & 3.7 & 7.9 & 3,000 & 16.9 & 18.2 \\
\hline 3309 & $\begin{array}{l}\text { Liquefied gas, toxic, flammable, corrosive, n.o.s. (Inhalation } \\
\text { Hazard Zone A) }\end{array}$ & 2,000 & 3.7 & 7.9 & 3,000 & 16.9 & 18.2 \\
\hline 3309 & $\begin{array}{l}\text { Liquefied gas, toxic, flammable, corrosive, n.o.s. (Inhalation } \\
\text { Hazard Zone B) }\end{array}$ & 100 & 0.1 & 0.6 & 1,400 & 2.5 & 6.7 \\
\hline
\end{tabular}


TABLE A.1 (Cont.)

\begin{tabular}{|c|c|c|c|c|c|c|c|}
\hline \multirow[b]{3}{*}{$\begin{array}{l}\text { UN ID } \\
\text { No. }\end{array}$} & \multirow[b]{3}{*}{ Name of Material } & \multicolumn{3}{|c|}{ Small Spills } & \multicolumn{3}{|c|}{ Large Spills } \\
\hline & & \multirow{2}{*}{$\begin{array}{c}\text { First } \\
\text { Isolate } \\
\text { in All } \\
\text { Direc- } \\
\text { tions (ft) }\end{array}$} & \multicolumn{2}{|c|}{$\begin{array}{l}\text { Then Protect } \\
\text { Persons } \\
\text { Downwind } \\
\text { during }\end{array}$} & \multirow{2}{*}{$\begin{array}{c}\text { First } \\
\text { Isolate } \\
\text { in All } \\
\text { Direc- } \\
\text { tions (ft) }\end{array}$} & \multicolumn{2}{|c|}{$\begin{array}{l}\text { Then Protect } \\
\text { Persons } \\
\text { Downwind } \\
\text { during }\end{array}$} \\
\hline & & & $\begin{array}{l}\text { Day } \\
(\mathrm{mi})\end{array}$ & $\begin{array}{l}\text { Night } \\
\text { (mi) }\end{array}$ & & $\begin{array}{l}\text { Day } \\
\text { (mi) }\end{array}$ & $\begin{array}{l}\text { Night } \\
(\mathrm{mi})\end{array}$ \\
\hline 3309 & $\begin{array}{l}\text { Liquefied gas, toxic, flammable, corrosive, n.o.s. (Inhalation } \\
\text { Hazard Zone C) }\end{array}$ & 100 & 0.1 & 0.5 & 800 & 1.5 & 4.0 \\
\hline 3309 & $\begin{array}{l}\text { Liquefied gas, toxic, flammable, corrosive, n.o.s. (Inhalation } \\
\text { Hazard Zone D) }\end{array}$ & 100 & 0.1 & 0.1 & 300 & 0.5 & 1.5 \\
\hline 3310 & Liquefied gas, poisonous, oxidizing, corrosive, n.o.s. & 2,000 & 3.7 & 7.9 & 3,000 & 16.9 & 18.2 \\
\hline 3310 & $\begin{array}{l}\text { Liquefied gas, poisonous, oxidizing, corrosive, n.o.s. } \\
\text { (Inhalation Hazard Zone A) }\end{array}$ & 2,000 & 3.7 & 7.9 & 3,000 & 16.9 & 18.2 \\
\hline 3310 & $\begin{array}{l}\text { Liquefied gas, poisonous, oxidizing, corrosive, n.o.s. } \\
\text { (Inhalation Hazard Zone B) }\end{array}$ & 200 & 0.3 & 1.3 & 1,200 & 2.2 & 5.5 \\
\hline 3310 & $\begin{array}{l}\text { Liquefied gas, poisonous, oxidizing, corrosive, n.o.s. } \\
\text { (Inhalation Hazard Zone C) }\end{array}$ & 100 & 0.2 & 0.8 & 800 & 1.5 & 4.0 \\
\hline 3310 & $\begin{array}{l}\text { Liquefied gas, poisonous, oxidizing, corrosive, n.o.s. } \\
\text { (Inhalation Hazard Zone D) }\end{array}$ & 100 & 0.1 & 0.4 & 200 & 0.4 & 1.4 \\
\hline 3310 & Liquefied gas, toxic, oxidizing, corrosive, n.o.s. & 2,000 & 3.7 & 7.9 & 3,000 & 16.9 & 18.2 \\
\hline 3310 & $\begin{array}{l}\text { Liquefied gas, toxic, oxidizing, corrosive, n.o.s. (Inhalation } \\
\text { Hazard Zone A) }\end{array}$ & 2,000 & 3.7 & 7.9 & 3,000 & 16.9 & 18.2 \\
\hline 3310 & $\begin{array}{l}\text { Liquefied gas, toxic, oxidizing, corrosive, n.o.s. (Inhalation } \\
\text { Hazard Zone B) }\end{array}$ & 200 & 0.3 & 1.3 & 1,200 & 2.2 & 5.5 \\
\hline 3310 & $\begin{array}{l}\text { Liquefied gas, toxic, oxidizing, corrosive, n.o.s. (Inhalation } \\
\text { Hazard Zone C) }\end{array}$ & 100 & 0.2 & 0.8 & 800 & 1.5 & 4.0 \\
\hline 3310 & $\begin{array}{l}\text { Liquefied gas, toxic, oxidizing, corrosive, n.o.s. (Inhalation } \\
\text { Hazard Zone D) }\end{array}$ & 100 & 0.1 & 0.4 & 200 & 0.4 & 1.4 \\
\hline 3318 & Ammonia solution, with more than $50 \%$ ammonia & 100 & 0.1 & 0.1 & 200 & 0.4 & 1.4 \\
\hline 3355 & Insecticide gas, poisonous, flammable, n.o.s & 400 & 0.8 & 3.2 & 3,000 & 5.4 & 9.6 \\
\hline 3355 & $\begin{array}{l}\text { Insecticide gas, poisonous, flammable, n.o.s. (Inhalation } \\
\text { Hazard Zone A) }\end{array}$ & 400 & 0.8 & 3.2 & 3,000 & 5.4 & 9.6 \\
\hline 3355 & $\begin{array}{l}\text { Insecticide gas, poisonous, flammable, n.o.s. (Inhalation } \\
\text { Hazard Zone B) }\end{array}$ & 100 & 0.2 & 0.8 & 1,400 & 2.5 & 6.7 \\
\hline 3355 & $\begin{array}{l}\text { Insecticide gas, poisonous, flammable, n.o.s. (Inhalation } \\
\text { Hazard Zone C) }\end{array}$ & 100 & 0.1 & 0.5 & 800 & 1.5 & 4.0 \\
\hline 3355 & $\begin{array}{l}\text { Insecticide gas, poisonous, flammable, n.o.s. (Inhalation } \\
\text { Hazard Zone D) }\end{array}$ & 100 & 0.1 & 0.1 & 300 & 0.5 & 1.5 \\
\hline 3355 & Insecticide gas, toxic, flammable, n.o.s & 400 & 0.8 & 3.2 & 3,000 & 5.4 & 9.6 \\
\hline 3355 & $\begin{array}{l}\text { Insecticide gas, toxic, flammable, n.o.s. (Inhalation Hazard } \\
\text { Zone A) }\end{array}$ & 400 & 0.8 & 3.2 & 3,000 & 5.4 & 9.6 \\
\hline 3355 & $\begin{array}{l}\text { Insecticide gas, toxic, flammable, n.o.s. (Inhalation Hazard } \\
\text { Zone B) }\end{array}$ & 100 & 0.2 & 0.8 & 1,400 & 2.5 & 6.7 \\
\hline 3355 & $\begin{array}{l}\text { Insecticide gas, toxic, flammable, n.o.s. (Inhalation Hazard } \\
\text { Zone C) }\end{array}$ & 100 & 0.1 & 0.5 & 800 & 1.5 & 4.0 \\
\hline 3355 & $\begin{array}{l}\text { Insecticide gas, toxic, flammable, n.o.s. (Inhalation Hazard } \\
\text { Zone D) }\end{array}$ & 100 & 0.1 & 0.1 & 300 & 0.5 & 1.5 \\
\hline 3381 & $\begin{array}{l}\text { Poisonous by inhalation liquid, n.o.s. (Inhalation Hazard } \\
\text { Zone A) }\end{array}$ & 500 & 0.8 & 2.2 & 3,000 & 9.9 & 12.4 \\
\hline
\end{tabular}


TABLE A.1 (Cont.)

\begin{tabular}{|c|c|c|c|c|c|c|c|}
\hline \multirow[b]{3}{*}{$\begin{array}{l}\text { UN ID } \\
\text { No. }\end{array}$} & \multirow[b]{3}{*}{ Name of Material } & \multicolumn{3}{|c|}{ Small Spills } & \multicolumn{3}{|c|}{ Large Spills } \\
\hline & & \multirow{2}{*}{$\begin{array}{c}\text { First } \\
\text { Isolate } \\
\text { in All } \\
\text { Direc- } \\
\text { tions (ft) }\end{array}$} & \multicolumn{2}{|c|}{$\begin{array}{l}\text { Then Protect } \\
\text { Persons } \\
\text { Downwind } \\
\text { during }\end{array}$} & \multirow{2}{*}{$\begin{array}{c}\text { First } \\
\text { Isolate } \\
\text { in All } \\
\text { Direc- } \\
\text { tions (ft) }\end{array}$} & \multicolumn{2}{|c|}{$\begin{array}{l}\text { Then Protect } \\
\text { Persons } \\
\text { Downwind } \\
\text { during }\end{array}$} \\
\hline & & & $\begin{array}{l}\text { Day } \\
(\mathrm{mi})\end{array}$ & $\begin{array}{l}\text { Night } \\
\text { (mi) }\end{array}$ & & $\begin{array}{l}\text { Day } \\
(\mathrm{mi})\end{array}$ & $\begin{array}{l}\text { Night } \\
(\mathrm{mi})\end{array}$ \\
\hline 3381 & Toxic by inhalation liquid, n.o.s. (Inhalation Hazard Zone A) & 500 & 0.8 & 2.2 & 3,000 & 9.9 & 12.4 \\
\hline 3382 & $\begin{array}{l}\text { Poisonous by inhalation liquid, n.o.s. (Inhalation Hazard } \\
\text { Zone B) }\end{array}$ & 200 & 0.3 & 1.1 & 1,100 & 2.1 & 4.6 \\
\hline 3382 & Toxic by inhalation liquid, n.o.s. (Inhalation Hazard Zone B) & 200 & 0.3 & 1.1 & 1,100 & 2.1 & 4.6 \\
\hline 3383 & $\begin{array}{l}\text { Poisonous by inhalation liquid, flammable, n.o.s. (Inhalation } \\
\text { Hazard Zone A) }\end{array}$ & 500 & 0.8 & 2.2 & 3,000 & 9.9 & 12.4 \\
\hline 3383 & $\begin{array}{l}\text { Toxic by inhalation liquid, flammable, n.o.s. (Inhalation } \\
\text { Hazard Zone A) }\end{array}$ & 500 & 0.8 & 2.2 & 3,000 & 9.9 & 12.4 \\
\hline 3384 & $\begin{array}{l}\text { Poisonous by inhalation liquid, flammable, n.o.s. (Inhalation } \\
\text { Hazard Zone B) }\end{array}$ & 200 & 0.2 & 0.6 & 900 & 1.6 & 3.5 \\
\hline 3384 & $\begin{array}{l}\text { Toxic by inhalation liquid, flammable, n.o.s. (Inhalation } \\
\text { Hazard Zone B) }\end{array}$ & 200 & 0.2 & 0.6 & 900 & 1.6 & 3.5 \\
\hline 3385 & $\begin{array}{l}\text { Poisonous by inhalation liquid, water-reactive, n.o.s. } \\
\text { (Inhalation Hazard Zone A) }\end{array}$ & 500 & 0.8 & 2.2 & 3,000 & 9.9 & 12.4 \\
\hline 3385 & $\begin{array}{l}\text { Toxic by inhalation liquid, water-reactive, n.o.s. (Inhalation } \\
\text { Hazard Zone A) }\end{array}$ & 500 & 0.8 & 2.2 & 3,000 & 9.9 & 12.4 \\
\hline 3386 & $\begin{array}{l}\text { Poisonous by inhalation liquid, water-reactive, n.o.s. } \\
\text { (Inhalation Hazard Zone B) }\end{array}$ & 200 & 0.3 & 1.1 & 1,100 & 2.1 & 4.6 \\
\hline 3386 & $\begin{array}{l}\text { Toxic by inhalation liquid, water-reactive, n.o.s. (Inhalation } \\
\text { Hazard Zone B) }\end{array}$ & 200 & 0.3 & 1.1 & 1,100 & 2.1 & 4.6 \\
\hline 3387 & $\begin{array}{l}\text { Poisonous by inhalation liquid, oxidizing, n.o.s. (Inhalation } \\
\text { Hazard Zone A) }\end{array}$ & 500 & 0.8 & 2.2 & 3,000 & 9.9 & 12.4 \\
\hline 3387 & $\begin{array}{l}\text { Toxic by inhalation liquid, oxidizing, n.o.s. (Inhalation } \\
\text { Hazard Zone A) }\end{array}$ & 500 & 0.8 & 2.2 & 3,000 & 9.9 & 12.4 \\
\hline 3388 & $\begin{array}{l}\text { Poisonous by inhalation liquid, oxidizing, n.o.s. (Inhalation } \\
\text { Hazard Zone B) }\end{array}$ & 100 & 0.2 & 0.9 & 900 & 1.7 & 4.3 \\
\hline 3388 & $\begin{array}{l}\text { Toxic by inhalation liquid, oxidizing, n.o.s. (Inhalation } \\
\text { Hazard Zone B) }\end{array}$ & 100 & 0.2 & 0.9 & 900 & 1.7 & 4.3 \\
\hline 3389 & $\begin{array}{l}\text { Poisonous by inhalation liquid, corrosive, n.o.s. (Inhalation } \\
\text { Hazard Zone A) }\end{array}$ & 300 & 0.5 & 1.5 & 2,500 & 3.9 & 7.5 \\
\hline 3389 & $\begin{array}{l}\text { Toxic by inhalation liquid, corrosive, n.o.s. (Inhalation } \\
\text { Hazard Zone A) }\end{array}$ & 300 & 0.5 & 1.5 & 2,500 & 3.9 & 7.5 \\
\hline 3390 & $\begin{array}{l}\text { Poisonous by inhalation liquid, corrosive, n.o.s. (Inhalation } \\
\text { Hazard Zone B) }\end{array}$ & 200 & 0.3 & 1.1 & 1,100 & 2.1 & 4.6 \\
\hline 3390 & $\begin{array}{l}\text { Toxic by inhalation liquid, corrosive, n.o.s. (Inhalation } \\
\text { Hazard Zone B) }\end{array}$ & 200 & 0.3 & 1.1 & 1,100 & 2.1 & 4.6 \\
\hline 3413 & Potassium cyanide, solution (when spilled in water) & 100 & 0.1 & 0.3 & 1,000 & 0.6 & 2.4 \\
\hline 3414 & Sodium cyanide, solution (when spilled in water) & 200 & 0.1 & 0.4 & 1,300 & 0.8 & 3.0 \\
\hline 3461 & Aluminum alkyl halides, solid (when spilled in water) & 100 & 0.1 & 0.1 & 100 & 0.2 & 0.8 \\
\hline 9191 & Chlorine dioxide, hydrate, frozen (when spilled in water) & 100 & 0.1 & 0.1 & 100 & 0.1 & 0.4 \\
\hline 9192 & Fluorine, refrigerated liquid (cryogenic liquid) & 100 & 0.1 & 0.3 & 300 & 0.5 & 2.2 \\
\hline 9202 & Carbon monoxide, refrigerated liquid (cryogenic liquid) & 100 & 0.1 & 0.1 & 300 & 0.4 & 1.5 \\
\hline 9206 & Methyl phosphonic dichloride & 100 & 0.1 & 0.1 & 100 & 0.1 & 0.1 \\
\hline
\end{tabular}


TABLE A.1 (Cont.)

\begin{tabular}{|c|c|c|c|c|c|c|c|}
\hline \multirow[b]{3}{*}{$\begin{array}{c}\text { UN ID } \\
\text { No. }\end{array}$} & \multirow[b]{3}{*}{ Name of Material } & \multicolumn{3}{|c|}{ Small Spills } & \multicolumn{3}{|c|}{ Large Spills } \\
\hline & & \multirow{2}{*}{$\begin{array}{c}\text { First } \\
\text { Isolate } \\
\text { in All } \\
\text { Direc- } \\
\text { tions (ft) }\end{array}$} & \multicolumn{2}{|c|}{$\begin{array}{l}\text { Then Protect } \\
\text { Persons } \\
\text { Downwind } \\
\text { during }\end{array}$} & \multirow{2}{*}{$\begin{array}{l}\text { First } \\
\text { Isolate } \\
\text { in All } \\
\text { Direc- } \\
\text { tions (ft) }\end{array}$} & \multicolumn{2}{|c|}{$\begin{array}{l}\text { Then Protect } \\
\text { Persons } \\
\text { Downwind } \\
\text { during }\end{array}$} \\
\hline & & & $\begin{array}{l}\text { Day } \\
\text { (mi) }\end{array}$ & $\begin{array}{l}\text { Night } \\
\text { (mi) }\end{array}$ & & $\begin{array}{l}\text { Day } \\
\text { (mi) }\end{array}$ & $\begin{array}{l}\text { Night } \\
\text { (mi) }\end{array}$ \\
\hline 9263 & Chloropivaloyl chloride & 100 & 0.1 & 0.1 & 100 & 0.2 & 0.3 \\
\hline 9264 & 3,5-Dichloro-2,4,6-trifluoropyridine & 100 & 0.1 & 0.1 & 100 & 0.2 & 0.3 \\
\hline 9269 & Trimethoxysilane & 100 & 0.1 & 0.3 & 400 & 0.7 & 1.4 \\
\hline
\end{tabular}

a The distances for MD (when used as a weapon) and methyldichloroarsine are transposed in the 2004 Emergency Response Guidebook. They are reported correctly in this document. 
APPENDIX B:

CHEMICALS ANALYZED FOR THE ERG2004 


\section{APPENDIX B:}

\section{CHEMICALS ANALYZED FOR THE ERG2004}

Table B.1 lists the chemicals analyzed for the 2004 Emergency Response Guidebook (ERG2004), in alphabetical order by DOT name. Most of these materials are TIH materials; however, several are surrogates for generic table entries (e.g., 2-amino-2-methylpropanenitrile) or mildly toxic components of mixtures (e.g., benzene, methyl chloride, etc). For reference, the CAS number, boiling point, vapor pressure at $20^{\circ} \mathrm{C}$, and toxicological data are provided. Additional chemical data used in the analysis include critical temperature, critical volume, and melting point and the temperature-dependent properties heat of vaporization, liquid density, specific heat of the liquid, viscosity, and surface tension. 
TABLE B.1 Chemicals Analyzed for the ERG2004 (see table endnotes for explanation of abbreviations)

\begin{tabular}{|c|c|c|c|c|c|c|c|c|}
\hline DOT Name & CAS \# & $\begin{array}{c}\text { Mol. } \\
\text { Weight }\end{array}$ & $\begin{array}{c}\text { Boiling } \\
\text { Point } \\
\left({ }^{\circ} \mathrm{C}\right) \\
\end{array}$ & $\begin{array}{c}\text { Vapor } \\
\text { Pressure at } \\
20^{\circ} \mathrm{C}(\mathrm{kPa})\end{array}$ & $\begin{array}{c}\mathrm{LC}_{50} \text { or } \mathrm{LC}_{\mathrm{LO}} \\
(\mathrm{ppm})\end{array}$ & $\begin{array}{c}\text { 1-hr } \\
\text { Protective } \\
(\mathrm{ppm})\end{array}$ & $\begin{array}{c}\text { 15-min } \\
\text { Protective } \\
(\mathrm{ppm})\end{array}$ & Basis \\
\hline Acrolein & $107-02-8$ & 56.1 & 52.7 & 29.69 & 62 & 0.5 & 1 & ERPG \\
\hline Acrylonitrile & $107-13-1$ & 53 & 77.4 & 11.4 & 666 & 35 & 70 & ERPG \\
\hline Aldicarb & $116-06-3$ & $\mathrm{~N} / \mathrm{A}$ & $\mathrm{N} / \mathrm{A}$ & N/A & 1 & 0.01 & 0.02 & $\mathrm{LC}_{50}$ \\
\hline Allyl alcohol & $107-18-6$ & 58.1 & 97.1 & 2.491 & 1,060 & 10 & 20 & $\mathrm{LC}_{50}$ \\
\hline Allyl chloroformate & $2937-50-0$ & 120.5 & 112.9 & 6.194 & 5 & 0.05 & 0.2 & $\mathrm{LC}_{50}$ \\
\hline Allyl isothiocyanate & $57-06-7$ & 99.2 & 150.7 & 0.516 & $28-\mathrm{S}$ & 1 & 2 & ERPG-S \\
\hline Allylamine & $107-11-9$ & 57.1 & 53.4 & 25.69 & 572 & 5 & 10 & $\mathrm{LC}_{50}$ \\
\hline 2-Amino-2-methylpropanenitrile & $19355-69-2$ & 84.1 & 159.5 & 0.099 & 112 & 1 & 2 & $\mathrm{LC}_{50}$ \\
\hline Ammonia & $7664-41-7$ & 17 & -33.5 & 854.5 & 7,338 & 150 & 300 & ERPG \\
\hline Arsenic trichloride & $7784-34-1$ & 181.2 & 130.1 & 1.113 & 56 & 0.5 & 1.0 & $\mathrm{LC}_{\mathrm{LO}}$ \\
\hline Arsine & $7784-42-1$ & 77.9 & -62.5 & 1475 & 30 & 0.5 & 0.6 & ERPG \\
\hline Benzene & $71-43-2$ & 78.1 & 80.1 & 9.983 & 26,458 & 150 & 300 & ERPG \\
\hline Bis-(2-chloroethyl) ethylamine & $538-07-8$ & 170.1 & 193.9 & 0.023 & 3.6 & 0.036 & 0.144 & $\mathrm{LC}_{50}$ \\
\hline Bis-(2-chloroethyl) methylamine & $51-75-2$ & 156.1 & 174.9 & 0.039 & 7.8 & 0.078 & 0.312 & $\mathrm{LC}_{50}$ \\
\hline Bis-(2-chloroethyl) sulfide & $505-60-2$ & 159.1 & 216.9 & 0.010 & 2.3 & 0.023 & 0.092 & $\mathrm{LC}_{50}$ \\
\hline Boron tribromide & $10294-33-4$ & 251.5 & 89.0 & 7.336 & $387-\mathrm{S}$ & 3 & 6 & $\mathrm{LC}_{50^{-}} \mathrm{S}$ \\
\hline Boron trichloride & $10294-34-5$ & 117.2 & 12.5 & 132.2 & 2541 & 25 & 50 & $\mathrm{LC}_{50}$ \\
\hline Boron trifluoride & $7637-07-2$ & 67.8 & -99.8 & 4,264 & 387 & 3 & 6 & ERPG \\
\hline Bromine & $7726-95-6$ & 159.8 & 58.8 & 22.87 & 310 & 1 & 2 & ERPG \\
\hline Bromine chloride & $13863-41-7$ & 115.4 & 4.9 & 220.0 & 290 & 3 & 6 & $\mathrm{LC}_{50}-\mathrm{S}$ \\
\hline Bromine pentafluoride & $7789-30-2$ & 174.9 & 40.9 & 42.88 & $299-S$ & 1 & 2 & ERPG-S \\
\hline Bromine trifluoride & $7787-71-5$ & 136.9 & 125.9 & 0.774 & $299-S$ & 1 & 2 & ERPG-S \\
\hline Bromoacetone & $598-31-2$ & 137 & 135.9 & 11.431 & 95 & 1 & 2 & $\mathrm{LC}_{\mathrm{LO}}$ \\
\hline n-Butyl chloroformate & $592-34-7$ & 136.6 & 137.9 & 0.765 & $323-\mathrm{S}$ & 3 & 6 & $\mathrm{LC}_{50}$ \\
\hline sec-Butyl chloroformate & $17462-58-7$ & 136.6 & 127.9-E & $1.051-\mathrm{E}$ & 323 & 3 & 6 & $\mathrm{LC}_{50^{-}} \mathrm{S}$ \\
\hline n-Butylisocyanate & $111-36-4$ & 99.1 & $115.0-\mathrm{S}$ & $1.755-\mathrm{S}$ & 28 & 0.05 & 0.1 & ERPG \\
\hline tert-Butyl-isocyanate & $1609-86-5$ & 99.1 & 86.0 & 4.888-E & 22 & 0.05 & 0.1 & ERPG-S \\
\hline tert-Butylarsine & $117791-53-4$ & 134 & 44.4-E & $40.35-\mathrm{E}$ & 140 & 1.5 & 3 & $\mathrm{LC}_{50}$ \\
\hline Carbon monoxide & $630-08-0$ & 28 & -191.5 & 2792 & 4,590 & 350 & 700 & ERPG \\
\hline Carbon tetrachloride & $56-23-5$ & 153.8 & 76.7 & 12.138 & 19,000 & 100 & 200 & ERPG \\
\hline Carbonyl fluoride & $353-50-4$ & 66 & -84.6 & 5211 & 360 & 3 & 6 & $\mathrm{LC}_{50}$ \\
\hline Carbonyl sulfide & $463-58-1$ & 60.1 & -50.2 & 1124 & 924 & 10 & 20 & $\mathrm{LC}_{50}$ \\
\hline Chlorine & $7782-50-5$ & 70.9 & -34.1 & 679.7 & 293 & 3 & 6 & ERPG \\
\hline Chlorine pentafluoride & $13637-63-3$ & 130.4 & -13.9 & 332.1 & 122 & 1 & 2 & ERPG-S \\
\hline Chlorine trifluoride & $7790-91-2$ & 92.4 & 11.8 & 148.0 & 299 & 1 & 2 & ERPG \\
\hline
\end{tabular}


TABLE B.1 (Cont.)

\begin{tabular}{|c|c|c|c|c|c|c|c|c|}
\hline DOT Name & CAS \# & $\begin{array}{c}\text { Mol. } \\
\text { Weight }\end{array}$ & $\begin{array}{c}\text { Boiling } \\
\text { Point } \\
\left({ }^{\circ} \mathrm{C}\right) \\
\end{array}$ & $\begin{array}{c}\text { Vapor } \\
\text { Pressure at } \\
20^{\circ} \mathrm{C}(\mathrm{kPa})\end{array}$ & $\begin{array}{c}\mathrm{LC}_{50} \text { or } \mathrm{LC}_{\mathrm{LO}} \\
(\mathrm{ppm})\end{array}$ & $\begin{array}{c}\text { 1-hr } \\
\text { Protective } \\
\text { (ppm) }\end{array}$ & $\begin{array}{c}\text { 15-min } \\
\text { Protective } \\
(\mathrm{ppm})\end{array}$ & Basis \\
\hline Chloroacetaldehyde & $107-20-0$ & 78.5 & 84.9 & 3.522 & 200 & 2 & 4 & $\mathrm{LC}_{50}$ \\
\hline Chloroacetone & $78-95-5$ & 92.5 & 120.1 & 1.567 & 262 & 2 & 4 & $\mathrm{LC}_{50}$ \\
\hline Chloroacetonitrile & $107-14-2$ & 75.5 & 126.0 & 1.087 & 500 & 5 & 10 & $\mathrm{LC}_{50}$ \\
\hline Chloroacetyl chloride & $79-04-9$ & 112.9 & 106.0 & 2.522 & 660 & 0.5 & 1 & ERPG \\
\hline Chloromethyl methyl ether & $107-30-2$ & 80.5 & 59.5 & 21.14 & 441 & 1 & 2 & ERPG \\
\hline p-Chlorophenyl isocyanate & $104-12-1$ & 153.6 & 199.0 & 0.069 & 18 & 0.3 & 0.6 & ERPG \\
\hline Chloropicrin & $76-06-2$ & 164.4 & 111.9 & 3.190 & 28 & 0.2 & 0.4 & ERPG \\
\hline Chloropivaloyl chloride & $4300-97-4$ & 155 & 147.9 & $0.189-\mathrm{E}$ & 126 & 1 & 2 & $\mathrm{LC}_{50}$ \\
\hline Chlorosulfonic acid & $7790-94-5$ & 116.5 & 153.9 & 0.309 & $929 \mathrm{mg} / \mathrm{m}^{3}$ & $10 \mathrm{mg} / \mathrm{m}^{3}$ & $20 \mathrm{mg} / \mathrm{m}^{3}$ & ERPG \\
\hline Crotonaldehyde & $4170-30-3$ & 70.1 & 104.9 & 3.121 & 380 & 10 & 20 & ERPG \\
\hline Cyanogen & $460-19-5$ & 52 & -21.2 & 489.7 & 350 & 3 & 6 & $\mathrm{LC}_{50}$ \\
\hline Cyanogen chloride & $506-77-4$ & 61.5 & 12.9 & 135.0 & 80 & 0.4 & 1.0 & ERPG \\
\hline Cyclohexyl isocyanate & $3173-53-3$ & 125.2 & 169.0 & 0.094 & 15 & 0.05 & 0.1 & ERPG-S \\
\hline Cyclohexyl methylphosphonofluoridate & $329-99-7$ & 180.2 & 238.9 & 0.006 & 0.08 & 0.0008 & 0.0032 & $\mathrm{LC}_{50}$ \\
\hline Diamylamine & $2050-92-2$ & 157.3 & 203.0 & 0.013 & 126 & 1 & 2 & $\mathrm{LC}_{\mathrm{LO}}$ \\
\hline Diborane & $19287-45-7$ & 27.7 & -92.6 & 3438 & 80 & 1 & 2 & ERPG \\
\hline Dichlorosilane & $4109-96-0$ & 101 & 8.4 & 154.2 & 215 & 2 & 4 & $\mathrm{LC}_{50}$ \\
\hline 3,5-Dichloro-2,4,6-trifluoropyridine & $1737-93-5$ & 202 & $177.5-\mathrm{S}$ & $0.104-\mathrm{S}$ & 62 & 0.5 & 1 & $\mathrm{LC}_{50}$ \\
\hline Diketene & $674-82-8$ & 84.1 & 126.1 & 1.064 & 612 & 5 & 10 & ERPG \\
\hline 1,1-Dimethyl hydrazine & $57-14-7$ & 60.1 & 63.4 & 16.38 & 1,410 & 15 & 30 & $\mathrm{LC}_{50}$ \\
\hline 1,2-Dimethyl hydrazine & $540-73-8$ & 60.1 & 87.1 & 7.230 & 680 & 7 & 15 & $\mathrm{LC}_{50}$ \\
\hline Dimethyl sulfate & $77-78-1$ & 126.1 & 188.9 & 0.069 & 17 & 0.15 & 0.3 & $\mathrm{LC}_{50}$ \\
\hline Diphosgene & $503-38-8$ & 197.8 & 127.9 & 0.553 & $50-\mathrm{S}$ & 0.1 & 0.2 & ERPG-S \\
\hline Ethyl chloroformate & $541-41-3$ & 108.5 & 92.9 & 2.121 & 145 & 1 & 2 & $\mathrm{LC}_{50}-\mathrm{S}$ \\
\hline Ethylchlorothioformate & $2812-73-9$ & 124.6 & 131.9 & $0.685-\mathrm{E}$ & $138-\mathrm{S}$ & 1 & 2 & $\mathrm{LC}_{50^{-}} \mathrm{S}$ \\
\hline Ethylchlorothiolformate & $2941-64-2$ & $124.6-\mathrm{S}$ & 131.9-S & $0.685-\mathrm{S}$ & $138-\mathrm{S}$ & 1 & 2 & $\mathrm{LC}_{50^{-}} \mathrm{S}$ \\
\hline Ethyl dichloroarsine & $598-14-1$ & 174.9 & 155.9 & 0.281 & 36 & 0.3 & 0.6 & $\mathrm{LC}_{50}$ \\
\hline Ethyl N,N-dimethylphosphoramidocyanidate & $77-81-6$ & 162.3 & 239.9-E & $0.005-\mathrm{E}$ & 0.18 & 0.0018 & 0.0072 & $\mathrm{LC}_{50}$ \\
\hline Ethyl isocyanate & $109-90-0$ & 71.1 & 61.6 & 24.50 & $28-\mathrm{S}$ & 0.5 & 1 & ERPG-S \\
\hline Ethyl phosphonothionic dichloride & $993-43-1$ & 162.9 & 176.9 & $0.026-\mathrm{E}$ & $62-\mathrm{S}$ & 0.5 & 1 & $\mathrm{LC}_{50}$ \\
\hline Ethyl phosphonous dichloride & $1498-40-4$ & 130.9 & 113.0-E & 4.762-E & 62 & 0.5 & 1 & $\mathrm{LC}_{\mathrm{LO}}$ \\
\hline Ethyl phosphorodichloridate & $1498-51-7$ & 162.9 & 166.9 & $0.040-\mathrm{E}$ & 43 & 0.3 & 0.6 & $\mathrm{LC}_{50}$ \\
\hline Ethylacrolein & $922-63-4$ & 84.1 & 92.9 & 5.392-E & 578 & 5 & 10 & $\mathrm{LC}_{50}$ \\
\hline Ethylene chlorohydrin & $107-07-3$ & 80.5 & 128.7 & 0.699 & 74 & 0.5 & 1 & $\mathrm{LC}_{50}$ \\
\hline Ethylene dibromide & $106-93-4$ & 187.9 & 131.4 & 1.357 & 691 & 5 & 10 & $\mathrm{LC}_{50}$ \\
\hline
\end{tabular}


TABLE B.1 (Cont.)

\begin{tabular}{|c|c|c|c|c|c|c|c|c|}
\hline DOT Name & CAS \# & $\begin{array}{c}\text { Mol. } \\
\text { Weight }\end{array}$ & $\begin{array}{c}\text { Boiling } \\
\text { Point } \\
\left({ }^{\circ} \mathrm{C}\right) \\
\end{array}$ & $\begin{array}{c}\text { Vapor } \\
\text { Pressure at } \\
20^{\circ} \mathrm{C}(\mathrm{kPa})\end{array}$ & $\begin{array}{c}\mathrm{LC}_{50} \text { or } \mathrm{LC}_{\mathrm{LO}} \\
(\mathrm{ppm})\end{array}$ & $\begin{array}{c}\text { 1-hr } \\
\text { Protective } \\
(\mathrm{ppm})\end{array}$ & $\begin{array}{c}\text { 15-min } \\
\text { Protective } \\
(\mathrm{ppm})\end{array}$ & Basis \\
\hline Ethylene oxide & $75-21-8$ & 44.1 & 10.5 & 146.3 & 5,840 & 50 & 100 & ERPG \\
\hline Ethylenimine & $151-56-4$ & 43.1 & 55.9 & 22.18 & 250 & 2 & 4 & $\mathrm{LC}_{50}$ \\
\hline Fluorine & $7782-41-4$ & 38 & -188.3 & 4160 & 185 & 5 & 15 & ERPG \\
\hline Germanium tetrachloride & 10038-98-9 & 214.4 & 83.9 & 9.51 & 150 & 1 & 2 & $\mathrm{LC}_{\mathrm{LO}}$ \\
\hline Germanium tetrahydride & $7782-65-2$ & 76.6 & -88.2 & 3870 & 622 & 5 & 10 & $\mathrm{LC}_{50}$ \\
\hline Hexachlorocyclopentadiene & $77-47-4$ & 272.8 & 239.1 & 0.0052 & 3 & 0.03 & 0.06 & $\mathrm{LC}_{50}$ \\
\hline Hexaethyltetraphosphate & $757-58-4$ & 506.2 & GS & GS & $24-\mathrm{E}$ & 0.5 & 1 & $\mathrm{LC}_{50}-\mathrm{E}$ \\
\hline Hexafluoroacetone & $684-16-2$ & 166 & -27.3 & 584.19 & 476 & 1 & 2 & ERPG \\
\hline Hydrogen bromide & $10035-10-6$ & 80.9 & -66.8 & 2182 & 2,860 & 20 & 40 & ERPG-S \\
\hline Hydrogen chloride & $7647-01-0$ & 36.5 & -85.1 & 4206 & 3,124 & 20 & 40 & ERPG \\
\hline Hydrogen cyanide & $74-90-8$ & 27 & 25.8 & 81.63 & 71 & 10 & 20 & ERPG \\
\hline Hydrogen fluoride & $7664-39-3$ & 20 & 19.6 & 102.7 & 1,300 & 20 & 40 & ERPG \\
\hline Hydrogen iodide & $10034-85-2$ & 127.9 & -35.6 & 691.0 & 2,860 & 20 & 40 & ERPG-S \\
\hline Hydrogen selenide & $7783-07-5$ & 81 & -42.1 & 911.1 & 5 & 0.2 & 0.4 & ERPG \\
\hline Hydrogen sulfide & $7783-06-4$ & 34.1 & -60.4 & 1781 & 712 & 30 & 60 & ERPG \\
\hline Iron pentacarbonyl & $13463-40-6$ & 195.9 & 102.8 & 3.142 & 57 & 0.5 & 1 & $\mathrm{LC}_{50}$ \\
\hline Isobutyl chloroformate & $543-27-1$ & 136.6 & 128.1 & 0.751 & 299 & 3 & 6 & $\mathrm{LC}_{50}$ \\
\hline Isobutyl isocyanate & $1873-29-6$ & 99.1 & 115.0 & 1.755 & $28-\mathrm{S}$ & 0.05 & 0.1 & ERPG-S \\
\hline Isopropyl chloroformate & $108-23-6$ & 122.6 & 104.9-S & 4.698-S & 299 & 3 & 6 & $\mathrm{LC}_{50}$ \\
\hline Isopropyl isocyanate & $1795-48-8$ & 85.1 & 82.9 & 7.372 & $28-\mathrm{S}$ & 0.05 & 0.1 & ERPG-S \\
\hline Isopropyl methylphosphonofluoridate & $107-44-8$ & 140.1 & 157.9 & 0.283 & 0.1 & 0.001 & 0.004 & $\mathrm{LC}_{50}$ \\
\hline Methacrylonitrile & $126-98-7$ & 67.1 & 90.4 & 7.541 & 656 & 5 & 10 & $\mathrm{LC}_{50}$ \\
\hline Methanesulfonyl monochloride & $124-63-0$ & 114.6 & 162.4 & 0.190 & $5-\mathrm{S}$ & 0.05 & 0.01 & $\mathrm{LC}_{50}-\mathrm{S}$ \\
\hline Methoxymethyl isocyanate & $6427-21-0$ & 87.1 & $165.7-\mathrm{E}$ & $0.250-\mathrm{E}$ & $28-\mathrm{S}$ & 0.05 & 0.1 & ERPG-S \\
\hline Methyl bromide & $74-83-9$ & 94.9 & 3.6 & 184.3 & 1007 & 50 & 100 & ERPG \\
\hline Methyl chloride & $74-87-3$ & 50.5 & -24.3 & 495.4 & 11,040 & 400 & 1,500 & ERPG \\
\hline Methyl chloroformate & $79-22-1$ & 94.5 & 70.9 & 11.20 & 88 & 1 & 2 & $\mathrm{LC}_{50}$ \\
\hline Methyl hydrazine & $60-34-4$ & 46.1 & 87.6 & 4.997 & 148 & 1 & 2 & $\mathrm{LC}_{50}$ \\
\hline Methyl iodide & $74-88-4$ & 141.9 & 42.5 & 44.33 & 1,600 & 50 & 100 & ERPG \\
\hline Methyl isocyanate & $624-83-9$ & 57.1 & 38.9 & 50.18 & 41 & 0.5 & 1 & ERPG \\
\hline Methyl isothiocyanate & $556-61-6$ & 73.1 & 118.9 & 3.205 & 635 & 5 & 10 & $\mathrm{LC}_{50}$ \\
\hline Methyl mercaptan & $74-93-1$ & 48.1 & 6.0 & 169.8 & 1,340 & 25 & 50 & ERPG \\
\hline Methyl phosphonic dichloride & $676-97-1$ & 132.9 & 162.9-E & $0.040-\mathrm{E}$ & 52 & 0.5 & 1.0 & $\mathrm{LC}_{50}$ \\
\hline Methyl phosphonous dichloride & $676-83-5$ & 116.9 & 81.9-E & 11.89-E & $52-\mathrm{S}$ & 0.5 & 1.0 & $\mathrm{LC}_{50}-\mathrm{S}$ \\
\hline Methyl silicate & $681-84-5$ & 152.2 & 120.9 & 1.613 & 300 & 3 & 6 & $\mathrm{LC}_{\mathrm{LO}}$ \\
\hline
\end{tabular}


TABLE B.1 (Cont.)

\begin{tabular}{|c|c|c|c|c|c|c|c|c|}
\hline DOT Name & CAS \# & $\begin{array}{c}\text { Mol. } \\
\text { Weight }\end{array}$ & $\begin{array}{c}\text { Boiling } \\
\text { Point } \\
\left({ }^{\circ} \mathrm{C}\right) \\
\end{array}$ & $\begin{array}{c}\text { Vapor } \\
\text { Pressure at } \\
20^{\circ} \mathrm{C}(\mathrm{kPa})\end{array}$ & $\begin{array}{c}\mathrm{LC}_{50} \text { or } \mathrm{LC}_{\mathrm{LO}} \\
(\mathrm{ppm})\end{array}$ & $\begin{array}{c}\text { 1-hr } \\
\text { Protective } \\
(\mathrm{ppm})\end{array}$ & $\begin{array}{c}\text { 15-min } \\
\text { Protective } \\
(\mathrm{ppm})\end{array}$ & Basis \\
\hline Methyl vinyl ketone & $78-94-4$ & 70.1 & 81.5 & 9.274 & 5 & 0.05 & 0.1 & $\mathrm{LC}_{50}$ \\
\hline Methylamine & $74-89-5$ & 31.1 & -6.3 & 295.7 & 5,000 & 100 & 200 & ERPG \\
\hline Methylchlorosilane & $993-00-0$ & 80.6 & 8.8 & 149.6 & $1547-\mathrm{S}$ & 3 & 6 & ERPG-S \\
\hline Methyldichloroarsine & $593-89-5$ & 160.9 & 135.9 & 1.034 & 68 & 0.5 & 1 & $\mathrm{LC}_{50}$ \\
\hline Nickel carbonyl & $13463-39-3$ & 170.8 & 42.5 & 43.50 & 18 & 0.15 & 0.3 & $\mathrm{LC}_{50}$ \\
\hline Nitric acid & $7697-37-2$ & 63 & 83.0 & 6.401 & 67 & 6 & 12 & ERPG \\
\hline Nitric oxide & $10102-43-9$ & 30 & -151.8 & 5093 & 1,708 & 15 & 30 & $\mathrm{LC}_{50}$ \\
\hline Nitrogen dioxide & $10102-44-0$ & 46 & 21.0 & 96.04 & 115 & 10 & 20 & EEL \\
\hline Nitrogen fluoride oxide & $13847-65-9$ & 87.1 & $-129.1-\mathrm{E}$ & 3979-E & 48 & 0.5 & 1.0 & $\mathrm{LC}_{50}$ \\
\hline Nitrogen trioxide & $10544-73-7$ & 76 & 2.0 & 218.3 & $115-\mathrm{S}$ & 10 & 20 & EEL \\
\hline Nitrosyl chloride & $2696-92-6$ & 65.5 & -5.3 & 270.4 & $293-\mathrm{S}$ & 3 & 6 & ERPG-S \\
\hline tert-octyl mercaptan & $141-59-3$ & 146.3 & 155.9 & 0.488 & 102 & 1 & 2 & $\mathrm{LC}_{50}$ \\
\hline O-Ethyl S-(2-diisopropylaminoethyl) methylphosphonothiolate & $50782-69-9$ & 267.4 & 297.9 & .00005 & 0.023 & 0.00023 & 0.0009 & $\mathrm{LC}_{50}$ \\
\hline Oxygen difluoride & $7783-41-7$ & 54 & -145.0 & 2789 & 2.6 & 0.03 & 0.06 & $\mathrm{LC}_{50}$ \\
\hline Parathion & $56-38-2$ & 291.3 & GS & GS & 14 & 0.15 & 0.3 & $\mathrm{LC}_{50}$ \\
\hline Pentaborane & $19624-22-7$ & 63.2 & 58.4 & $22.70-\mathrm{E}$ & 10 & 0.1 & 0.2 & $\mathrm{LC}_{50}$ \\
\hline Perchloromethyl mercaptan & $594-42-3$ & 185.9 & 148.0 & 0.642 & 69 & 0.5 & 1.0 & $\mathrm{LC}_{50}$ \\
\hline Perchloryl fluoride & $7616-94-6$ & 102.4 & -46.7 & 1060 & 770 & 5 & 10 & $\mathrm{LC}_{50}$ \\
\hline Phenyl isocyanate & $103-71-9$ & 119.1 & 165.7 & 0.250 & 16 & 0.05 & 0.1 & ERPG-S \\
\hline Phenyl mercaptan & $108-98-5$ & 110.2 & 169.2 & 0.142 & 66 & 0.5 & 1.0 & $\mathrm{LC}_{50}$ \\
\hline Phosgene & $75-44-5$ & 98.9 & 7.6 & 159.3 & 5 & 0.2 & 0.4 & ERPG \\
\hline Phosphine & $7803-51-2$ & 34 & -87.8 & 3517 & 22 & 0.5 & 1 & ERPG \\
\hline Phosphorous oxychloride & $10025-87-3$ & 153.3 & 105.5 & 3.273 & 96 & 1 & 2 & $\mathrm{LC}_{50}$ \\
\hline Phosphorous pentafluoride & $7647-19-0$ & 126 & -84.6 & 56888 & 260 & 2 & 4 & $\mathrm{LC}_{50}-\mathrm{S}$ \\
\hline Phosphorous trichloride & $7719-12-2$ & 137.3 & 76.1 & 12.82 & 208 & 2 & 4 & $\mathrm{LC}_{50}$ \\
\hline Phosphorous trifluoride & $7783-55-3$ & 88 & -101.3 & 6902 & 433 & 3 & 6 & $\mathrm{LC}_{50}$ \\
\hline Pinacolyl methylphosphonofluoridate & $96-64-0$ & 182.2 & 197.9 & 0.037 & 0.08 & 0.0008 & 0.0032 & $\mathrm{LC}_{50}$ \\
\hline n-Propyl chloroformate & $109-61-5$ & 122.6 & 104.9 & 4.698 & 319 & 3 & 2 & $\mathrm{LC}_{50}$ \\
\hline n-Propyl isocyanate & $110-78-1$ & 85.1 & 82.9 & 5.274 & 44 & 0.05 & 0.1 & ERPG-S \\
\hline Selenium hexafluoride & $7783-79-1$ & 193 & -34.7 & 2854 & 50 & 0.5 & 1 & $\mathrm{LC}_{50}$ \\
\hline Silicon tetrafluoride & $7783-61-1$ & 104.1 & -95.2 & 3205 & 922 & 10 & 20 & $\mathrm{LC}_{50}$ \\
\hline Stibine & $7803-52-3$ & 124.8 & -18.5 & 286.1-E & 20 & 0.5 & 1 & ERPG \\
\hline Sulfur chloride pentafluoride & $13780-57-9$ & 162.5 & -21.2 & $442.0-\mathrm{S}$ & 100 & 1 & 2 & $\mathrm{LC}_{\mathrm{LO}}$ \\
\hline Sulfur dioxide & $7446-09-5$ & 64.1 & -10.1 & 336.5 & 2,520 & 3 & 6 & ERPG \\
\hline Sulfur monochloride & $10025-67-9$ & 135 & 137.9 & 0.944 & 150 & 1 & 2 & $\mathrm{LC}_{50}$ \\
\hline
\end{tabular}


TABLE B.1 (Cont.)

\begin{tabular}{|c|c|c|c|c|c|c|c|c|}
\hline DOT Name & CAS \# & $\begin{array}{c}\text { Mol. } \\
\text { Weight }\end{array}$ & $\begin{array}{c}\text { Boiling } \\
\text { Point } \\
\left({ }^{\circ} \mathrm{C}\right)\end{array}$ & $\begin{array}{c}\text { Vapor } \\
\text { Pressure at } \\
20^{\circ} \mathrm{C}(\mathrm{kPa})\end{array}$ & $\begin{array}{c}\mathrm{LC}_{50} \text { or } \mathrm{LC}_{\mathrm{LO}} \\
(\mathrm{ppm})\end{array}$ & $\begin{array}{c}\begin{array}{c}1-\mathrm{hr} \\
\text { Protective } \\
(\mathrm{ppm})\end{array} \\
\end{array}$ & $\begin{array}{c}\text { 15-min } \\
\text { Protective } \\
(\mathrm{ppm})\end{array}$ & Basis \\
\hline Sulfur tetrafluoride & $7783-60-0$ & 108.1 & -40.4 & 1785 & 40 & 0.4 & 0.8 & $\mathrm{LC}_{50}$ \\
\hline Sulfur trioxide & $7446-11-9$ & 80.1 & 44.8 & 25.73 & $106 \mathrm{mg} / \mathrm{m}^{3}$ & $10 \mathrm{mg} / \mathrm{m}^{3}$ & $20 \mathrm{mg} / \mathrm{m}^{3}$ & ERPG \\
\hline Sulfuryl chloride & $7791-25-5$ & 135 & 69.4 & 14.811 & $3,020-\mathrm{S}$ & 30 & 60 & $\mathrm{LC}_{50}-\mathrm{S}$ \\
\hline Sulfuryl fluoride & $2699-79-8$ & 102.1 & -55.4 & 1964 & 3,020 & 30 & 60 & $\mathrm{LC}_{50}$ \\
\hline Tellurium hexafluoride & $7783-80-4$ & 241.6 & -38.2 & 709.6 & 20 & 0.2 & 0.4 & $\mathrm{LC}_{\mathrm{LO}}$ \\
\hline Tetraethyl dithiopyrophosphate & $3689-24-5$ & 322.3 & GS & GS & 1.5 & 0.015 & 0.02 & $\mathrm{LC}_{50}$ \\
\hline Tetraethyl pyrophosphate & $107-49-3$ & 290.1 & GS & GS & $1.5-\mathrm{S}$ & 0.015 & 0.02 & $\mathrm{LC}_{50}-\mathrm{S}$ \\
\hline Tetrafluorohydrazine & $10036-47-2$ & 104 & -74.3 & 2515 & 950 & 10 & 20 & $\mathrm{LC}_{50}$ \\
\hline Tetramethyl tin & $594-27-4$ & 178.8 & 77.9 & 17.92-E & 58 & 0.6 & 1.2 & $\mathrm{LC}_{\mathrm{LO}}$ \\
\hline Tetranitromethane & $509-14-8$ & 196 & 125.8 & 1.121 & 36 & 0.3 & 0.6 & $\mathrm{LC}_{50}$ \\
\hline Thionyl chloride & $7719-09-7$ & 119 & 75.7 & 12.77 & 500 & 2 & 4 & ERPG \\
\hline Thiophosgene & $463-71-8$ & 115 & 72.9 & 15.04 & $25-\mathrm{S}$ & 0.2 & 0.4 & ERPG-S \\
\hline Titanium tetrachloride & $7550-45-0$ & 189.7 & 135.9 & 1.253 & $1,300 \mathrm{mg} / \mathrm{m}^{3}$ & $20 \mathrm{mg} / \mathrm{m}^{3}$ & $40 \mathrm{mg} / \mathrm{m}^{3}$ & ERPG \\
\hline Trichloroacetyl chloride & $76-02-8$ & 181.8 & 118.0 & 2.189 & 128 & 1 & 2 & ERPG-S \\
\hline Trifluoroacetyl chloride & $354-32-5$ & 132.5 & -17.9 & $356.2-\mathrm{S}$ & 208 & 1 & 2 & ERPG-S \\
\hline Trifluorochloroethylene & $79-38-9$ & 116.5 & -27.9 & 531.4 & 8,568 & 100 & 200 & ERPG \\
\hline Trimethoxy silane & $2487-90-3$ & 122.3 & 80.9 & $20.40-\mathrm{E}$ & 84 & 2 & 4 & ERPG \\
\hline Trimethylacetyl chloride & $3282-30-2$ & 120.6 & 106.9 & 2.866 & 250 & 5 & 10 & $\mathrm{LC}_{50}$ \\
\hline Tris-(2-chloroethyl) amine & $817-09-4$ & 204.5 & 255.9 & 0.00094 & 3 & 0.03 & 0.12 & $\mathrm{LC}_{50}$ \\
\hline Tungsten hexafluoride & $7783-82-6$ & 297.8 & 17.4 & 111.8 & 207 & 2 & 4 & $\mathrm{LC}_{50}$ \\
\hline
\end{tabular}

$\underline{\text { Abbreviations }}$

ERPG Emergency Response Planning Guideline, established by the AIHA

EEL Emergency Exposure Level, published in the AIHA Journal

$\mathrm{LC}_{50} \quad$ Median lethal concentration; lethal to $50 \%$ of a population exposed via inhalation

$\mathrm{LC}_{\mathrm{LO}} \quad$ Lowest lethal concentration reported in an animal study

$\mathrm{LC}_{50}-\mathrm{E} \quad \mathrm{LC}_{50}$ estimated from oral toxicity data

E Estimated value

GS Solid or liquid in solution with gas

S Data are for a structurally similar chemical 


\section{APPENDIX C:}

EXPERIMENTAL PROGRAM CONDUCTED TO SUPPORT THE WATER REACTIVITY ANALYSIS 


\section{APPENDIX C:}

\section{EXPERIMENTAL PROGRAM CONDUCTED TO SUPPORT THE WATER REACTIVITY ANALYSIS}

Few data exist in the chemical literature on the rate of evolution of $\mathrm{TIH}$ gases from the reactions between water and different substances. In 1999, we began a program of gas-evolution experiments to create a basis for estimates of how fast different water-reactive substances emit TIH gases when they are spilled into water. Two series of experiments have been performed. The 1999 series covered 21 substances in support of the ERG2000. Some of these experiments were intended to rule out certain substances as TIHWR substances. The 2003 series covered 35 additional substances in support of the ERG2004 and repeated and extended the observations on 10 substances from the first group.

\section{C.1 GENERAL DESCRIPTION}

\section{C.1.1 Apparatus and Experimental Method}

A small amount of a substance was mixed with water in a closed system. If a reaction occurred and generated a gas, the system expanded. Changes in the volume of the system were noted as functions of time. From these data, the mass of the gas that the reaction generated and the percentage yield of the gas could be calculated as functions of time. The first of these results required only the chemical formula of the gas; the second required a balanced chemical equation representing the gas-generating reaction.

Figure C.1 provides a diagram of the experimental apparatus. The system was first purged with dry nitrogen. Reactions were performed using 1.00-mmol (millimole) or 2.00-mmol samples of the water-reactive substances as received from vendors. Liquid chemicals of known density were measured out by volume. Different orders of mixing and amounts of waters were used (see method A and method B below), but the sample/water mixtures were in every case stirred continuously at a constant rate (using a magnetic stirrer). The evolution of gases in the closed apparatus displaced a manometric liquid (octane in Figure C.1) upward into the burette, and the increase in volume was noted at appropriate intervals of time. The burette was canted upward from the horizontal at an angle of about 15 degrees. This arrangement allowed the volume of the system to increase substantially while limiting the increase in the interior pressure to a negligible amount (less than 10-15 cm of manometric liquid, or about $0.015 \mathrm{~atm}$ ). Care was taken to use a manometric liquid that did not react with the $\mathrm{TIH}$ gas. When gaseous $\mathrm{HCl}$ was evolved, for example, the manometric liquid was octane saturated with $\mathrm{HCl}$. This precaution prevented loss of a TIH gas by dissolution in the manometric liquid or reaction with it. The experiments were carried out at room temperature $\left(24^{\circ} \mathrm{C}\right)$. Although the reactions were all exothermic, the small volume of the reaction mixture favored fast cooling; the temperature of the system generally remained close to room temperature. 


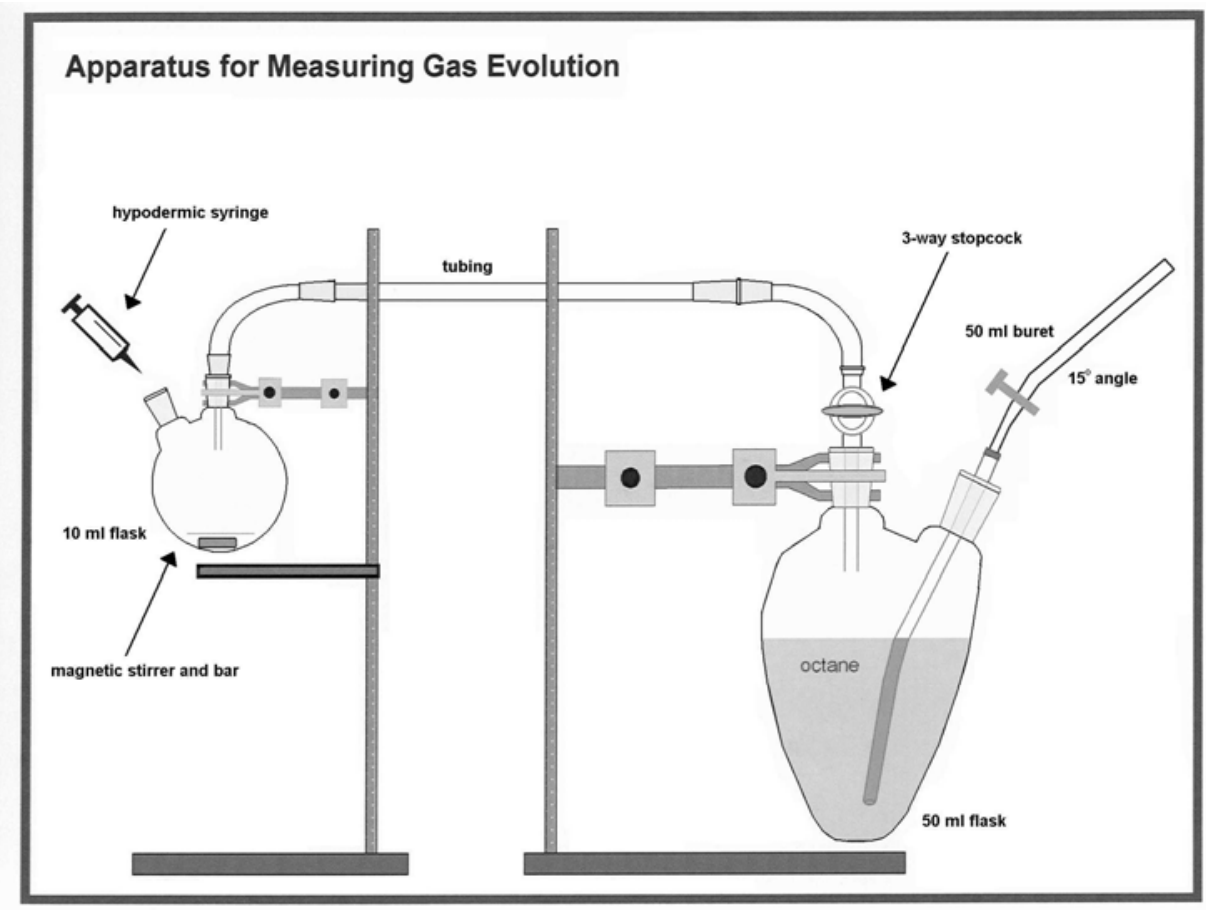

FIGURE C.1 Apparatus Used to Measure the Amounts and Rates of Evolution of Gases When Substances React with Water

The gas-evolution apparatus was calibrated by reacting $0.149 \mathrm{~g}(2.00 \mathrm{mmol})$ of potassium chloride $(\mathrm{KCl})$ with excess $98 \%$ sulfuric acid $\left(\mathrm{H}_{2} \mathrm{SO}_{4}\right)$.

$$
2 \mathrm{KCl}(\mathrm{s})+\mathrm{H}_{2} \mathrm{SO}_{4}(\mathrm{aq}) \longrightarrow 2 \mathrm{HCl}(\mathrm{g})+\mathrm{K}_{2} \mathrm{SO}_{4}(\mathrm{aq})
$$

The final volume of $\mathrm{HCl}(\mathrm{g})$ (gaseous hydrogen chloride) generated in the apparatus equaled $32.0 \mathrm{~mL}$ at $24^{\circ} \mathrm{C}$ and $1.0 \mathrm{~atm}$. This volume was taken as equivalent to $2.00 \mathrm{mmol}$ of gas, which is the theoretical yield of $\mathrm{HCl}(\mathrm{g})$ according to the above equation.

The masses of the gases that were produced in the apparatus at various times after the first mixing of the reactants were calculated using this equivalence and the molar masses of the gases. The calculations thus assumed that during the course of the experiments the gases were confined under the same temperature and pressure as $\mathrm{HCl}(\mathrm{g})$ was confined at the end of the calibration run. The calculations also assumed that all gases (actually, gas mixtures) in the apparatus behaved ideally.

The experiments were carried out in two different ways:

- Method A: After the nitrogen purge, $1.00 \mathrm{mmol}$ (typically) of substance was injected into the reaction flask through a side-arm covered with a rubber cap. The stirrer was started, and a chemically equivalent amount of water, as determined from the chemical equation written to represent the anticipated 
reaction, was rapidly injected. The clock was started when the water was first added. For example, $1.00 \mathrm{mmol}(0.170 \mathrm{~g})$ of tetrachlorosilane $\left(\mathrm{SiCl}_{4}\right)$ was charged into the flask, and $0.036 \mathrm{~g}(2.00 \mathrm{mmol})$ of $\mathrm{H}_{2} \mathrm{O}$ was added. This $1: 2$ molar ratio ensured that both reactants would be consumed entirely if the reaction

$$
\mathrm{SiCl}_{4}+2 \mathrm{H}_{2} \mathrm{O} \longrightarrow 4 \mathrm{HCl}+\mathrm{SiO}_{2}
$$

went to completion. Method A was designed to verify the evolution of TIH gases, to measure their yield, and to estimate the rate of their production.

- Method B: The substance was added to the water (rather than the reverse), and a fivefold molar excess of water was used. For example, $10.0 \mathrm{mmol} \mathrm{of} \mathrm{H}_{2} \mathrm{O}$

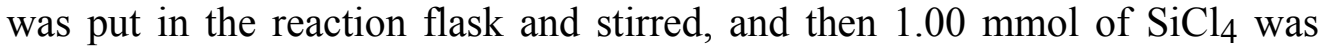
rapidly injected. This method was intended to model an actual spill more realistically.

\section{C.1.2 Notes on Hydrogen Chloride}

Hydrogen chloride $(\mathrm{HCl})$ is a $\mathrm{TIH}$ gas that is evolved from reactions between water and a number of commercially important substances. Some of its physical properties and its interactions with water accordingly merit discussion.

Hydrogen chloride has a normal boiling point of $-85.05^{\circ} \mathrm{C}$ and is therefore gaseous under ambient conditions of temperature and pressure. It dissolves readily in water to give $\mathrm{HCl}(\mathrm{aq})$, or aqueous hydrogen chloride, more commonly called hydrochloric acid. The solubility of $\mathrm{HCl}$ in water is large but not unlimited. It depends upon the temperature and also upon on the partial pressure of gaseous $\mathrm{HCl}$ (symbolized $P_{\mathrm{HCl}}$ ) above the solution as described by Henry's law

$$
X_{\mathrm{HCl}}=K_{\mathrm{H}} P_{\mathrm{HCl}}
$$

where $K_{\mathrm{H}}$ is a temperature-dependent constant (the Henry's law constant) and $X_{\mathrm{HCl}}$ is the mole fraction of $\mathrm{HCl}$ in the solution

$$
X_{\mathrm{HCl}}=\frac{n_{\mathrm{HCl}}}{n_{\mathrm{HCl}}+n_{\mathrm{H}_{2} \mathrm{O}}} .
$$

The Henry's law constant for $\mathrm{HCl}$ in $\mathrm{H}_{2} \mathrm{O}$ at $25^{\circ} \mathrm{C}$ is $0.258 \mathrm{~atm}^{-1}$. This constant changes with temperature: from 0.289 at $0^{\circ} \mathrm{C}$ to 0.217 at $60^{\circ} \mathrm{C}$, for example. The decrease in $K_{\mathrm{H}}$ with increasing temperature means that $\mathrm{HCl}$ is less soluble in hot $\mathrm{H}_{2} \mathrm{O}$ than in cold. This solubility behavior is typical of gases. 
It can be shown that the mass fraction (symbolized $w_{\mathrm{HCl}}$ ) of $\mathrm{HCl}$ in saturated binary $\mathrm{HCl} / \mathrm{H}_{2} \mathrm{O}$ mixtures is related to the mole fraction $X_{\mathrm{HCl}}$ as follows

$$
\frac{1}{w_{\mathrm{HCl}}}=\left(1-\frac{18.02}{36.46}\right)+\left(\frac{18.02}{36.46}\right) \cdot \frac{1}{X_{\mathrm{HCl}}},
$$

where 36.46 and 18.02 are the relative molar masses of $\mathrm{HCl}$ and $\mathrm{H}_{2} \mathrm{O}$, respectively. If $\mathrm{H}_{2} \mathrm{O}$ is saturated with $\mathrm{HCl}$ at a partial pressure of 1 atm at $25^{\circ} \mathrm{C}$, then

$$
\begin{aligned}
& X_{\mathrm{HCl}}=\left(0.258 \mathrm{~atm}^{-1}\right)(1 \mathrm{~atm})=0.258 \text { and } \\
& w_{\mathrm{HCl}}=0.413 .
\end{aligned}
$$

At $24^{\circ} \mathrm{C}$, the temperature at which the experiments described in this report were performed, the result is essentially the same.

The concentrated hydrochloric acid of commerce contains somewhat less than $41 \% \mathrm{HCl}$ by mass. It has a nominal composition of $37.5 \% \mathrm{HCl}$ and varies between about 36.5 and $38 \%$. Closed bottles of concentrated $\mathrm{HCl}(\mathrm{aq})$ emit fumes of $\mathrm{HCl}(\mathrm{g})$ when they are opened to the air because the partial pressure of $\mathrm{HCl}(\mathrm{g})$ in the atmosphere is $0 . \mathrm{A} 41 \%$ aqueous solution of $\mathrm{HCl}$ fumes heavily when opened to the air. The fumes appear white in moist air because the escaping $\mathrm{HCl}(\mathrm{g})$ interacts with the water vapor to give $\mathrm{HCl}(\mathrm{aq})$ droplets of colloidal size. Fumes of $\mathrm{HCl}(\mathrm{g})$ are colorless in dry air.

Water and $\mathrm{HCl}$ form a non-ideal solution. The attractions between $\mathrm{HCl}$ and $\mathrm{H}_{2} \mathrm{O}$ molecules in the mixture are stronger than either the $\mathrm{HCl}$-to- $\mathrm{HCl}$ or $\mathrm{H}_{2} \mathrm{O}$-to- $\mathrm{H}_{2} \mathrm{O}$ attractions. One consequence is the existence of a high-boiling-point azeotrope. (An azeotrope is a liquid solution for which the equilibrium composition of vapors confined above the solution is the same as the composition of the solution itself.) Experiments show that the composition of the $\mathrm{HCl} / \mathrm{H}_{2} \mathrm{O}$ binary azeotrope is $w_{\mathrm{HCl}}=0.2022$ (corresponding to $X_{\mathrm{HCl}}=0.1113$ ). Evaporative loss from an azeotrope does not change the composition of the remaining solution. When $\mathrm{HCl} / \mathrm{H}_{2} \mathrm{O}$ solutions in which $w_{\mathrm{HCl}}$ exceeds 0.2022 evaporate at $25^{\circ} \mathrm{C}$, they lose $\mathrm{HCl}(\mathrm{g})$, the more volatile component, preferentially until $w_{\mathrm{HCl}}$ falls to 0.2022 . Then, $w_{\mathrm{HCl}}$ stays at 0.2022 until the whole of the solution has evaporated. When $\mathrm{HCl} / \mathrm{H}_{2} \mathrm{O}$ solutions in which $w_{\mathrm{HCl}}$ is less than 0.2022 evaporate, they lose $\mathrm{H}_{2} \mathrm{O}$ preferentially, despite the fact that $\mathrm{H}_{2} \mathrm{O}$ is less volatile than $\mathrm{HCl}$ at all temperatures, until $w_{\mathrm{HCl}}$ rises to 0.2022 .

In Method B in our experiments on HCl-generating chlorosilanes, the equilibrium composition of the aqueous phase should lie between $w_{\mathrm{HCl}}=0.20$ and $w_{\mathrm{HCl}}=0.41$ if the additional components in the system can be neglected. These components are the nitrogen in the gaseous phase above the solution and the by-products of the $\mathrm{HCl}$-producing reaction. The latter are often, but not always, insoluble polymeric materials. Dissolution of these materials in the aqueous phase would in principle influence the amount of $\mathrm{HCl}$ that is co-dissolved. 


\section{C.2 EXPERIMENTAL RESULTS}

The following tables give the observed changes in the volume of the experimental system as functions of the time after different water-reactive substances were mixed with water. The tables also include the yields of the TIH gases as functions of time, both in terms of their mass and as percentages of the theoretical yield. The computations of this last quantity required a balanced chemical equation, which is given. Notes on variations in procedures and particular observations and some brief discussion are also given.

For ease of reference, the chemicals are presented in order of UN identification number and correspond to those chemicals listed in Table D.1 for which experiments were conducted.

\section{UN 1162 - Dimethydichlorosilane, $\left(\mathrm{CH}_{3}\right)_{2} \mathrm{SiCl}_{2}$}

Dimethydichlorosilane is a liquid with a relative molar mass of 129.06 and a roomtemperature density of $1.064 \mathrm{~g} / \mathrm{cm}^{3}$. In method A, $1.00 \mathrm{mmol}$ of it was mixed with $1.00 \mathrm{mmol}$ of water. In method B, $1.00 \mathrm{mmol}$ of it was mixed with $5.00 \mathrm{mmol}$ of water. Reaction according to the equation

$$
\left(\mathrm{CH}_{3}\right)_{2} \mathrm{SiCl}_{2}+\mathrm{H}_{2} \mathrm{O} \longrightarrow 2 \mathrm{HCl}+\left[\left(\mathrm{CH}_{3}\right)_{2} \mathrm{SiO}\right]_{\mathrm{n}} \text { polymer }
$$

was taken as the source of $\mathrm{HCl}(\mathrm{g})$. The theoretical yield of $\mathrm{HCl}(\mathrm{g})$ was $2.00 \mathrm{mmol}$, which corresponded to $32.0 \mathrm{~mL}$ of gas in the calibrated experimental apparatus.

1999 Experimental Results for Dimethydichlorosilane

\begin{tabular}{|c|c|c|c|c|c|c|c|}
\hline \multicolumn{4}{|c|}{ Method A } & \multicolumn{4}{c|}{ Method B } \\
\hline $\begin{array}{c}\text { Time } \\
(\mathrm{min})\end{array}$ & $\begin{array}{c}\text { Volume of } \\
\text { Gas (mL) }\end{array}$ & $\begin{array}{c}\text { Mass of } \\
\mathrm{HCl}(\mathrm{g})\end{array}$ & $\begin{array}{c}\text { Yield } \\
(\%)\end{array}$ & $\begin{array}{c}\text { Time } \\
(\mathrm{min})\end{array}$ & $\begin{array}{c}\text { Volume of } \\
\text { Gas }(\mathrm{mL})\end{array}$ & $\begin{array}{c}\text { Mass of } \\
\mathrm{HCl}(\mathrm{g})\end{array}$ & $\begin{array}{c}\text { Yield } \\
(\%)\end{array}$ \\
\hline 0 & 0 & 0 & 0 & 0 & 0 & 0 & 0 \\
\hline 1 & 5 & 0.0113 & 16 & 1 & 8 & 0.0182 & 25 \\
\hline 5 & 19 & 0.043 & 59 & 5 & 12 & 0.027 & 37 \\
\hline 10 & 30 & 0.068 & 94 & 10 & 13 & 0.030 & 40 \\
\hline 20 & 32 & 0.073 & 100 & 20 & 13 & 0.030 & 40 \\
\hline
\end{tabular}


2003 Experimental Results for Dimethydichlorosilane

\begin{tabular}{|c|c|c|c|c|c|c|c|}
\hline \multicolumn{4}{|c|}{ Method A } & \multicolumn{4}{c|}{ Method B } \\
\hline $\begin{array}{c}\text { Time } \\
(\mathrm{min})\end{array}$ & $\begin{array}{c}\text { Volume of } \\
\text { Gas (mL) }\end{array}$ & $\begin{array}{c}\text { Mass of } \\
\mathrm{HCl}(\mathrm{g})\end{array}$ & $\begin{array}{c}\text { Yield } \\
(\%)\end{array}$ & $\begin{array}{c}\text { Time } \\
(\mathrm{min})\end{array}$ & $\begin{array}{c}\text { Volume of } \\
\text { Gas (mL) }\end{array}$ & $\begin{array}{c}\text { Mass of } \\
\mathrm{HCl}(\mathrm{g})\end{array}$ & $\begin{array}{c}\text { Yield } \\
(\%)\end{array}$ \\
\hline 0 & 0 & 0 & 0 & 0 & 0 & 0 & 0 \\
\hline- & - & - & - & 0.5 & 5.9 & 0.0134 & 18.4 \\
\hline 1 & 5.0 & 0.0114 & 15.6 & 1 & 7.8 & 0.0178 & 24.4 \\
\hline 2 & 9.2 & 0.0210 & 28.8 & 2 & 9.6 & 0.0219 & 30.0 \\
\hline 3 & 12.9 & 0.0294 & 40.3 & 3 & 10.6 & 0.024 & 33.1 \\
\hline 4 & 16.3 & 0.037 & 50.9 & 4 & 11.3 & 0.026 & 35.3 \\
\hline 5 & 19.4 & 0.044 & 60.6 & 5 & 11.8 & 0.027 & 36.9 \\
\hline 7 & 24.2 & 0.055 & 75.6 & 7 & 12.5 & 0.028 & 39.1 \\
\hline 10 & 29.7 & 0.068 & 92.8 & 10 & 12.8 & 0.029 & 40.0 \\
\hline 20 & 31.6 & 0.072 & 98.8 & 20 & 13.2 & 0.030 & 41.3 \\
\hline 30 & 32.0 & 0.073 & 100.0 & 30 & 13.3 & 0.030 & 41.6 \\
\hline
\end{tabular}

Comments: The experiments were repeated in 2003 to obtain more data on the early course of the reaction. In method $\mathrm{A}$, the full theoretical amount of $\mathrm{HCl}(\mathrm{g})$ formed in $30 \mathrm{~min}$; in method B, a solid by-product formed immediately upon mixing the reactants. This by-product might have sequestered some of the test substance, some of the water, or both. If, however, we suppose that the reaction was complete after $30 \mathrm{~min}$ in method $\mathrm{B}$, then $(1-0.416) \times 2.00 \mathrm{mmol}$ of $\mathrm{HCl}$ was dissolved in $4.00 \mathrm{mmol}$ of excess water. Such a solution has a mole fraction of $\mathrm{HCl}$ $\left(X_{\mathrm{HCl}}\right)$ of $1.17 \div(1.17+4.00)$, or 0.226 . This is fairly close to but still somewhat less than the concentration of $\mathrm{HCl}$ in saturated $\mathrm{HCl} / \mathrm{H}_{2} \mathrm{O}$ solutions at $24^{\circ} \mathrm{C}$ (see Section C.1.2).

\section{UN 1196 - Ethyltrichlorosilane, $\mathrm{CH}_{3} \mathrm{CH}_{2} \mathrm{SiCl}_{3}$}

Ethyltrichlorosilane is a liquid with a relative molar mass of 163.51 and a roomtemperature density of $1.238 \mathrm{~g} / \mathrm{cm}^{3}$. In method A, $1.00 \mathrm{mmol}$ of it was mixed with $1.50 \mathrm{mmol}$ of water. In method B, $1.00 \mathrm{mmol}$ of it was mixed with $7.50 \mathrm{mmol}$ of water. The equation

$$
\mathrm{CH}_{3} \mathrm{CH}_{2} \mathrm{SiCl}_{3}+3 / 2 \mathrm{H}_{2} \mathrm{O} \longrightarrow 3 \mathrm{HCl}+\left[\mathrm{CH}_{3} \mathrm{CH}_{2} \mathrm{SiO}_{3 / 2}\right]_{\mathrm{n}} \text { polymer }
$$

was taken to represent the evolution of gas. The theoretical yield of $\mathrm{HCl}(\mathrm{g})(3.00 \mathrm{mmol})$ corresponded to $48.0 \mathrm{~mL}$ of gas in the calibrated experimental apparatus. 
2003 Experimental Results for Ethyltrichlorosilane

\begin{tabular}{|c|c|c|c|c|c|c|c|}
\hline \multicolumn{4}{|c|}{ Method A } & \multicolumn{4}{c|}{ Method B } \\
\hline $\begin{array}{c}\text { Time } \\
(\mathrm{min})\end{array}$ & $\begin{array}{c}\text { Volume of } \\
\text { Gas (mL) }\end{array}$ & $\begin{array}{c}\text { Mass of } \\
\mathrm{HCl}(\mathrm{g})\end{array}$ & $\begin{array}{c}\text { Yield } \\
(\%)\end{array}$ & $\begin{array}{c}\text { Time } \\
(\mathrm{min})\end{array}$ & $\begin{array}{c}\text { Volume of } \\
\text { Gas (mL) }\end{array}$ & $\begin{array}{c}\text { Mass of } \\
\mathrm{HCl}(\mathrm{g})\end{array}$ & $\begin{array}{c}\text { Yield } \\
(\%)\end{array}$ \\
\hline 1 & 5.1 & 0.012 & 10.6 & 1 & 12.5 & 0.028 & 26.0 \\
\hline 2 & 11.2 & 0.026 & 23.3 & 2 & 21.2 & 0.048 & 44.2 \\
\hline 3 & 19. & 0.044 & 40.2 & 3 & 28.1 & 0.064 & 58.5 \\
\hline 4 & 27.5 & 0.063 & 57.3 & 4 & 32.1 & 0.073 & 66.9 \\
\hline 5 & 33.6 & 0.077 & 70.0 & 5 & 31.5 & 0.072 & 65.6 \\
\hline 6 & 40.5 & 0.106 & 84.0 & 6 & 28.6 & 0.065 & 59.6 \\
\hline 11 & 48.0 & 0.109 & 100 & 11 & 26.7 & 0.061 & 55.6 \\
\hline 15 & 48.0 & 0.109 & 100 & 15 & 24.0 & 0.055 & 50.0 \\
\hline & & & & 20 & 22.3 & 0.151 & 46.5 \\
\hline & & & & 30 & 18.5 & 0.042 & 38.5 \\
\hline & & & & 120 & 18.1 & 0.041 & 37.7 \\
\hline
\end{tabular}

Comments: In method $\mathrm{A}$, the theoretical amount of $\mathrm{HCl}(\mathrm{g})$ was evolved while a white solid by-product (a polysiloxane) formed. In method $\mathrm{B}, \mathrm{HCl}(\mathrm{g})$ was evolved as a similar white solid formed. The amount of $\mathrm{HCl}(\mathrm{g})$ in method $\mathrm{B}$ went to a maximum and then diminished. This was caused by the dissolution of some of the $\mathrm{HCl}(\mathrm{g})$ in the excess water. The by-product might have sequestered some of the test substance, some of the water, or both. If, however, we suppose that the $\mathrm{HCl}$-generating reaction was complete after $120 \mathrm{~min}$ in method $\mathrm{B}$, then $(1-0.377) \times$ $3.00 \mathrm{mmol}$ of $\mathrm{HCl}$ was dissolved in $6.00 \mathrm{mmol}$ of excess water. Such a solution has a mole fraction of $\mathrm{HCl}\left(X_{\mathrm{HCl}}\right)$ of $1.87 \div(1.87+6.00)$, or 0.237 . This is fairly close to but still somewhat less than the concentration of $\mathrm{HCl}$ in saturated $\mathrm{HCl} / \mathrm{H}_{2} \mathrm{O}$ solutions at $24^{\circ} \mathrm{C}$ (see Section C.1.2).

\section{UN 1242 - Methyldichlorosilane, $\left(\mathrm{CH}_{3}\right) \mathrm{SiHCl}_{2}$}

Methyldichlorosilane is a liquid with relative molar mass of 115.04 and a roomtemperature density of $1.105 \mathrm{~g} / \mathrm{cm}^{3}$. In method A, $1.00 \mathrm{mmol}$ of it was mixed with $1.00 \mathrm{mmol}$ of water. In method B, $1.00 \mathrm{mmol}$ of it was mixed with $5.00 \mathrm{mmol}$ of water. Reaction according to the equation

$$
\left(\mathrm{CH}_{3}\right) \mathrm{HSiCl}_{2}+\mathrm{H}_{2} \mathrm{O} \longrightarrow 2 \mathrm{HCl}+\left[\left(\mathrm{CH}_{3}\right) \mathrm{HSiO}\right]_{\mathrm{n}} \text { polymer }
$$

was taken as the source of $\mathrm{HCl}(\mathrm{g})$. The theoretical yield of $\mathrm{HCl}(\mathrm{g})$ was $2.00 \mathrm{mmol}$, which corresponded to $32.0 \mathrm{~mL}$ of gas in the calibrated experimental apparatus. 
2003 Experimental Data for Methyldichlorosilane

\begin{tabular}{|c|c|c|c|c|c|c|c|}
\hline \multicolumn{4}{|c|}{ Method A } & \multicolumn{4}{c|}{ Method B } \\
\hline $\begin{array}{c}\text { Time } \\
(\mathrm{min})\end{array}$ & $\begin{array}{c}\text { Volume of } \\
\text { Gas }(\mathrm{mL})\end{array}$ & $\begin{array}{c}\text { Mass of } \\
\mathrm{HCl}(\mathrm{g})\end{array}$ & $\begin{array}{c}\text { Yield } \\
(\%)\end{array}$ & $\begin{array}{c}\text { Time } \\
(\mathrm{min})\end{array}$ & $\begin{array}{c}\text { Volume of } \\
\text { Gas (mL) }\end{array}$ & $\begin{array}{c}\text { Mass of } \\
\mathrm{HCl}(\mathrm{g})\end{array}$ & $\begin{array}{c}\text { Yield } \\
(\%)\end{array}$ \\
\hline 0.33 & 10.1 & 0.023 & 31.6 & 0.33 & 18.3 & 0.042 & 57.2 \\
\hline 0.67 & 14.5 & 0.033 & 45.3 & 0.67 & 19.2 & 0.044 & 60.0 \\
\hline 1 & 18.9 & 0.043 & 59.1 & 1 & 18.5 & 0.042 & 57.8 \\
\hline 2 & 19.8 & 0.045 & 61.9 & 2 & 18.1 & 0.041 & 56.6 \\
\hline 3 & 21.6 & 0.049 & 67.5 & 3 & 17.5 & 0.040 & 54.7 \\
\hline 5 & 21.8 & 0.05 & 68.1 & 5 & 16.8 & 0.038 & 52.5 \\
\hline 10 & 21.9 & 0.050 & 68.4 & 7 & 16.5 & 0.038 & 51.6 \\
\hline & & & & 10 & 16.3 & 0.037 & 50.9 \\
\hline & & & & 20 & 16.2 & 0.037 & 50.6 \\
\hline
\end{tabular}

Comments: In method $\mathrm{A}$, an immediate reaction very rapidly formed a viscous liquid polysiloxane. This product might have sequestered some water and so restricted the yield of $\mathrm{HCl}(\mathrm{g})$. In method B, reaction was also quick; a viscous liquid polysiloxane formed as $\mathrm{HCl}(\mathrm{g})$ evolved. A maximum on the $\mathrm{HCl}(\mathrm{g})$ yield curve was apparent.

\section{UN 1250 - Methyltrichlorosilane, $\mathrm{CH}_{3} \mathrm{SiCl}_{3}$}

Methyltrichlorosilane is a liquid with a relative molar mass of 149.48 and a roomtemperature density of $1.273 \mathrm{~g} / \mathrm{cm}^{3}$. In method A, $2.00 \mathrm{mmol}$ (instead of $1.00 \mathrm{mmol}$ ) of it was mixed with $3.00 \mathrm{mmol}$ of water. In method B, $2.00 \mathrm{mmol}$ of it was mixed with $10.0 \mathrm{mmol}$ of water. Reaction according to the equation

$$
\mathrm{CH}_{3} \mathrm{SiCl}_{3}+3 / 2 \mathrm{H}_{2} \mathrm{O} \longrightarrow 3 \mathrm{HCl}+\left[\mathrm{CH}_{3} \mathrm{SiO}_{3 / 2}\right]_{\mathrm{n}} \text { polymer }
$$

was taken as the source of $\mathrm{HCl}(\mathrm{g})$. The theoretical yield of $\mathrm{HCl}(\mathrm{g})(6.00 \mathrm{mmol})$ corresponded to $96.0 \mathrm{~mL}$ of gas in the calibrated experimental apparatus.

1999 Experimental Results for Methyltrichlorosilane

\begin{tabular}{|c|c|c|c|c|c|c|c|}
\hline \multicolumn{4}{|c|}{ Method A } & \multicolumn{4}{c|}{ Method B } \\
\hline $\begin{array}{c}\text { Time } \\
(\mathrm{min})\end{array}$ & $\begin{array}{c}\text { Volume of } \\
\text { Gas }(\mathrm{mL})\end{array}$ & $\begin{array}{c}\text { Mass of } \\
\mathrm{HCl}(\mathrm{g})\end{array}$ & $\begin{array}{c}\text { Yield } \\
(\%)\end{array}$ & $\begin{array}{c}\text { Time } \\
(\mathrm{min})\end{array}$ & $\begin{array}{c}\text { Volume of } \\
\text { Gas (mL) }\end{array}$ & $\begin{array}{c}\text { Mass of } \\
\mathrm{HCl}(\mathrm{g})\end{array}$ & $\begin{array}{c}\text { Yield } \\
(\%)\end{array}$ \\
\hline 1 & 19 & 0.023 & 10 & 1 & 22 & 0.050 & 23 \\
\hline 5 & 18 & 0.041 & 19 & 5 & 28 & 0.064 & 29 \\
\hline 10 & 24 & 0.055 & 25 & 10 & 30 & 0.068 & 31 \\
\hline 20 & 26 & 0.059 & 27 & 20 & 32 & 0.073 & 33 \\
\hline
\end{tabular}


2003 Experimental Results for Methyltrichlorosilane

\begin{tabular}{|c|c|c|c|c|c|c|c|}
\hline \multicolumn{4}{|c|}{ Method A } & \multicolumn{4}{c|}{ Method B } \\
\hline $\begin{array}{c}\text { Time } \\
(\mathrm{min})\end{array}$ & $\begin{array}{c}\text { Volume of } \\
\text { Gas }(\mathrm{mL})\end{array}$ & $\begin{array}{c}\text { Mass of } \\
\mathrm{HCl}(\mathrm{g})\end{array}$ & $\begin{array}{c}\text { Yield } \\
(\%)\end{array}$ & $\begin{array}{c}\text { Time } \\
(\mathrm{min})\end{array}$ & $\begin{array}{c}\text { Volume of } \\
\text { Gas }(\mathrm{mL})\end{array}$ & $\begin{array}{c}\text { Mass of } \\
\mathrm{HCl}(\mathrm{g})\end{array}$ & $\begin{array}{c}\text { Yield } \\
(\%)\end{array}$ \\
\hline 0.5 & 5.8 & 0.0132 & 6.0 & 0.5 & 15.5 & 0.035 & 16.1 \\
\hline 1 & 8.9 & 0.0203 & 9.3 & 1 & 22.0 & 0.050 & 22.9 \\
\hline 2 & 11.7 & 0.0267 & 12.2 & 2 & 24.2 & 0.055 & 25.2 \\
\hline 3 & 14.1 & 0.032 & 14.7 & 3 & 26.8 & 0.061 & 27.9 \\
\hline 4 & 16.1 & 0.037 & 16.8 & 4 & 27.4 & 0.063 & 28.5 \\
\hline 5 & 17.8 & 0.041 & 18.5 & 5 & 28.0 & 0.064 & 29.2 \\
\hline 7 & 20.3 & 0.046 & 21.1 & 7 & 29.1 & 0.066 & 30.3 \\
\hline 10 & 23.7 & 0.054 & 24.7 & 10 & 30.5 & 0.070 & 31.8 \\
\hline 20 & 25.8 & 0.059 & 26.9 & 20 & 32.5 & 0.074 & 33.9 \\
\hline 30 & 26.2 & 0.060 & 27.3 & 30 & 32.8 & 0.075 & 34.2 \\
\hline 45 & 26.5 & 0.060 & 27.6 & 45 & 33.0 & 0.075 & 34.4 \\
\hline
\end{tabular}

Comments: The 1999 experiments were repeated in 2003 to obtain more data on the early course of the reaction. Only $27-28 \%$ of the theoretical amount of $\mathrm{HCl}(\mathrm{g})$ was generated with method A. A white solid formed immediately after the reactants were mixed. This byproduct, a polymeric siloxane, probably sequestered $\mathrm{H}_{2} \mathrm{O}$ or unreacted methyltrichlorosilane. A similar white solid was observed with method B. No maxima were observed in the $\mathrm{HCl}(\mathrm{g})$ yield curves with method B. The absence of such maxima does not however imply that dissolution of $\mathrm{HCl}(\mathrm{g})$ fails to occur.

\section{UN 1298 - Trimethylchlorosilane, $\left(\mathrm{CH}_{3}\right)_{3} \mathrm{SiCl}$}

Trimethylchlorosilane is a liquid with a relative molar mass of 108.64 and a roomtemperature density of $1.257 \mathrm{~g} / \mathrm{cm}^{3}$. In method A, $1.00 \mathrm{mmol}$ of it was mixed with $1.00 \mathrm{mmol}$ of water. In method B, $1.00 \mathrm{mmol}$ was mixed with $5.00 \mathrm{mmol}$ of water. Reaction according to the equation

$$
\left(\mathrm{CH}_{3}\right)_{3} \mathrm{SiCl}+\mathrm{H}_{2} \mathrm{O} \longrightarrow \mathrm{HCl}+\left[\left(\mathrm{CH}_{3}\right)_{3} \mathrm{SiOH}\right]_{\mathrm{n}}
$$

was taken as the source of $\mathrm{HCl}(\mathrm{g})$ for computations of mass and yield. The theoretical yield of $\mathrm{HCl}(\mathrm{g})$ was $1.00 \mathrm{mmol}$, which corresponded to $16.0 \mathrm{~mL}$ of gas in the calibrated experimental apparatus. 
1999 Experimental Results for Trimethylchlorosilane

\begin{tabular}{|c|c|c|c|c|c|c|c|}
\hline \multicolumn{4}{|c|}{ Method A } & \multicolumn{4}{c|}{ Method B } \\
\hline $\begin{array}{c}\text { Time } \\
(\mathrm{min})\end{array}$ & $\begin{array}{c}\text { Volume of } \\
\text { Gas }(\mathrm{mL})\end{array}$ & $\begin{array}{c}\text { Mass of } \\
\mathrm{HCl}(\mathrm{g})\end{array}$ & $\begin{array}{c}\text { Yield } \\
(\%)\end{array}$ & $\begin{array}{c}\text { Time } \\
(\mathrm{min})\end{array}$ & $\begin{array}{c}\text { Volume of } \\
\text { Gas }(\mathrm{mL})\end{array}$ & $\begin{array}{c}\text { Mass of } \\
\mathrm{HCl}(\mathrm{g})\end{array}$ & $\begin{array}{c}\text { Yield } \\
(\%)\end{array}$ \\
\hline 1 & 2 & 0.0046 & 13 & 1 & 4.5 & 0.0102 & 28 \\
\hline 5 & 4.5 & 0.0102 & 28 & 5 & 5.5 & 0.0125 & 34 \\
\hline 10 & 5 & 0.0114 & 31 & 10 & 6 & 0.0137 & 38 \\
\hline 20 & 5.5 & 0.0125 & 34 & 20 & 6 & 0.0137 & 38 \\
\hline
\end{tabular}

2003 Experimental Results for Trimethylchlorosilane

\begin{tabular}{|c|c|c|c|c|c|c|c|}
\hline \multicolumn{4}{|c|}{ Method A } & \multicolumn{4}{c|}{ Method B } \\
\hline $\begin{array}{c}\text { Time } \\
(\mathrm{min})\end{array}$ & $\begin{array}{c}\text { Volume of } \\
\text { Gas }(\mathrm{mL})\end{array}$ & $\begin{array}{c}\text { Mass of } \\
\mathrm{HCl}(\mathrm{g})\end{array}$ & $\begin{array}{c}\text { Yield } \\
(\%)\end{array}$ & $\begin{array}{c}\text { Time } \\
(\mathrm{min})\end{array}$ & $\begin{array}{c}\text { Volume of } \\
\text { Gas }(\mathrm{mL})\end{array}$ & $\begin{array}{c}\text { Mass of } \\
\mathrm{HCl}(\mathrm{g})\end{array}$ & $\begin{array}{c}\text { Yield } \\
(\%)\end{array}$ \\
\hline 0.5 & 1.3 & 0.0030 & 8.1 & 0.5 & 3.1 & 0.0071 & 19.4 \\
\hline 1 & 2.1 & 0.0048 & 13.1 & 1 & 4.2 & 0.0096 & 26.3 \\
\hline 2 & 2.9 & 0.0066 & 18.1 & 2 & 5.1 & 0.0116 & 31.9 \\
\hline 3 & 3.6 & 0.0082 & 22.5 & 3 & 5.3 & 0.0121 & 33.1 \\
\hline 4 & 4.1 & 0.0093 & 25.6 & 4 & 5.6 & 0.0128 & 35.0 \\
\hline 5 & 4.6 & 0.0105 & 28.8 & 5 & 5.8 & 0.0132 & 36.3 \\
\hline 10 & 5.2 & 0.0118 & 32.5 & 10 & 6.2 & 0.0141 & 38.8 \\
\hline 20 & 5.7 & 0.0130 & 35.6 & 20 & 6.4 & 0.0146 & 40.0 \\
\hline 30 & 5.9 & 0.0134 & 36.9 & 30 & 6.5 & 0.0148 & 40.6 \\
\hline 45 & 6.0 & 0.0137 & 37.5 & 45 & 6.6 & 0.0150 & 41.3 \\
\hline
\end{tabular}

Comments: The 1999 experiments were repeated in 2003 to obtain more data on the early course of the reaction. In method $\mathrm{A}$, the reaction produced a white solid by-product and only about one-third of the theoretical amount of $\mathrm{HCl}(\mathrm{g})$. In method $\mathrm{B}$, the white solid byproduct also appeared. The by-product might have sequestered some of the $\mathrm{HCl}$ in both cases. No dissolution of $\mathrm{HCl}(\mathrm{g})$ in excess water was observed towards the end of the reaction [i.e., there was no maximum on the $\mathrm{HCl}(\mathrm{g})$ yield curve].

\section{UN 1305 - Vinyltrichlorosilane, $\mathrm{CH}_{2}=\mathrm{CHSiCl}_{3}$}

Vinyltrichlorosilane is a liquid with a relative molar mass of 161.49 and a roomtemperature density of $1.270 \mathrm{~g} / \mathrm{cm}^{3}$. In method A, $1.00 \mathrm{mmol}$ of it was mixed with $1.50 \mathrm{mmol}$ of water. In method B, $1.00 \mathrm{mmol}$ of it was mixed with $7.50 \mathrm{mmol}$ of water. The equation

$$
\mathrm{CH}_{2} \mathrm{CHSiCl}_{3}+3 / 2 \mathrm{H}_{2} \mathrm{O} \longrightarrow 3 \mathrm{HCl}+\left[\mathrm{CH}_{2} \mathrm{CHSiO}_{3} / 2\right]_{\mathrm{n}} \text { polymer }
$$


was taken to represent the evolution of gas. The theoretical yield of $\mathrm{HCl}(\mathrm{g})$ (3.00 mmol) corresponded to $48.0 \mathrm{~mL}$ of gas in the calibrated experimental apparatus.

\begin{tabular}{|c|c|c|c|c|c|c|c|}
\hline \multicolumn{8}{|c|}{2003 Experimental Results for Vinyltrichlorosilane } \\
\hline \multicolumn{4}{|c|}{ Method A } & \multicolumn{4}{|c|}{ Method B } \\
\hline $\begin{array}{l}\text { Time } \\
\text { (min) }\end{array}$ & $\begin{array}{l}\text { Volume of } \\
\text { Gas (mL) }\end{array}$ & $\begin{array}{l}\text { Mass of } \\
\mathrm{HCl}(\mathrm{g})\end{array}$ & $\begin{array}{c}\text { Yield } \\
(\%)\end{array}$ & $\begin{array}{l}\text { Time } \\
\text { (min) }\end{array}$ & $\begin{array}{l}\text { Volume of } \\
\text { Gas (mL) }\end{array}$ & $\begin{array}{l}\text { Mass of } \\
\mathrm{HCl}(\mathrm{g})\end{array}$ & $\begin{array}{l}\text { Yield } \\
(\%)\end{array}$ \\
\hline 1 & 2.6 & 0.006 & 5.4 & 1 & 22.3 & 0.051 & 46.5 \\
\hline 2 & 4.0 & 0.009 & 8.3 & 2 & 27.1 & 0.062 & 56.5 \\
\hline 3 & 4.7 & 0.011 & 9.8 & 3 & 26.3 & 0.060 & 54.8 \\
\hline 4 & 5.1 & 0.012 & 10.6 & 4 & 25.6 & 0.058 & 53.3 \\
\hline 5 & 5.4 & 0.012 & 11.3 & 5 & 25.0 & 0.057 & 52.1 \\
\hline 6 & 5.7 & 0.013 & 11.9 & 6 & 24.2 & 0.055 & 50.4 \\
\hline 8 & 6.1 & 0.014 & 12.7 & 8 & 23.1 & 0.053 & 48.1 \\
\hline 10 & 6.5 & 0.015 & 13.5 & 10 & 21.9 & 0.050 & 45.6 \\
\hline 12 & 6.8 & 0.015 & 14.2 & 12 & 21.0 & 0.048 & 43.8 \\
\hline 15 & 7.1 & 0.016 & 14.8 & 15 & 19.8 & 0.045 & 41.3 \\
\hline 20 & 7.6 & 0.017 & 15.8 & 20 & 18.0 & 0.041 & 37.5 \\
\hline 25 & 8.1 & 0.018 & 16.9 & 25 & 16.9 & 0.039 & 35.2 \\
\hline 30 & 8.6 & 0.020 & 17.9 & 30 & 15.8 & 0.036 & 32.9 \\
\hline 45 & 9.4 & 0.021 & 19.6 & 45 & 15.1 & 0.034 & 31.5 \\
\hline 60 & 10.1 & 0.023 & 21.0 & 60 & 14.7 & 0.034 & 30.6 \\
\hline 90 & 10.9 & 0.025 & 22.7 & 90 & 14.5 & 0.033 & 30.2 \\
\hline 120 & 11.4 & 0.026 & 23.8 & 120 & 14.3 & 0.033 & 29.8 \\
\hline 180 & 11.6 & 0.026 & 24.2 & 180 & 14.1 & 0.032 & 29.4 \\
\hline
\end{tabular}

Comments: In method A, a white solid polysiloxane quickly formed. This by-product apparently sequestered some water and so prevented completion of the reaction. Some $\mathrm{HCl}$ was also conceivably consumed by a side-reaction involving the vinyl group. In method B, the lessthan-theoretical yield of $\mathrm{HCl}(\mathrm{g})$ was ascribed to sequestration of some reactant by the polymeric by-product and dissolution of $\mathrm{HCl}(\mathrm{g})$ in the excess water.

\section{UN 1360 - Calcium Phosphide, $\mathrm{Ca}_{3} \mathrm{P}_{2}$}

Calcium phosphide is a solid with a relative molar mass of 182.18. In method A, $6.00 \mathrm{mmol}$ of water was added to $1.00 \mathrm{mmol}$ of it. In method B, $1.00 \mathrm{mmol}$ of it was added to $30.0 \mathrm{mmol}$ of water. The reaction

$$
\mathrm{Ca}_{3} \mathrm{P}_{2}+6 \mathrm{H}_{2} \mathrm{O} \longrightarrow 2 \mathrm{PH}_{3}+3 \mathrm{Ca}(\mathrm{OH})_{2}
$$

was taken as the source of gaseous phosphine $\left(\mathrm{PH}_{3}\right)$. Generation of $2.00 \mathrm{mmol}^{\circ} \mathrm{PH}_{3}(\mathrm{~g})$ corresponded to an increase in volume of $32.0 \mathrm{~mL}$ in the calibrated apparatus. Pure octane was used as the manometric fluid. 
1999 Experimental Results for Calcium Phosphide

\begin{tabular}{|c|c|c|c|c|c|c|c|}
\hline \multicolumn{4}{|c|}{ Method A } & \multicolumn{4}{c|}{ Method B } \\
\hline $\begin{array}{c}\text { Time } \\
(\mathrm{min})\end{array}$ & $\begin{array}{c}\text { Volume of } \\
\text { Gas }(\mathrm{mL})\end{array}$ & $\begin{array}{c}\text { Mass of } \\
\mathrm{PH}_{3}(\mathrm{~g})\end{array}$ & $\begin{array}{c}\text { Yield } \\
(\%)\end{array}$ & $\begin{array}{c}\text { Time } \\
(\mathrm{min})\end{array}$ & $\begin{array}{c}\text { Volume of } \\
\text { Gas (mL) }\end{array}$ & $\begin{array}{c}\text { Mass of } \\
\mathrm{PH}_{3}(\mathrm{~g})\end{array}$ & $\begin{array}{c}\text { Yield } \\
(\%)\end{array}$ \\
\hline 1 & 1.0 & 0.00212 & 3.1 & 1 & 1.9 & 0.00425 & 6.2 \\
\hline 2 & 2.0 & 0.00425 & 6.2 & 2 & 4.9 & 0.0106 & 15.6 \\
\hline 5 & 2.0 & 0.00425 & 6.2 & 5 & 8.0 & 0.0170 & 25.0 \\
\hline 10 & 2.0 & 0.00425 & 6.2 & 10 & 9.0 & 0.0191 & 28.1 \\
\hline 20 & 2.0 & 0.00425 & 6.2 & 20 & 9.0 & 0.0191 & 28.1 \\
\hline
\end{tabular}

2003 Experimental Results for Calcium Phosphide

\begin{tabular}{|c|c|c|c|c|c|c|c|}
\hline \multicolumn{4}{|c}{ Method A } & \multicolumn{4}{c|}{ Method B } \\
\hline $\begin{array}{c}\text { Time } \\
(\mathrm{min})\end{array}$ & $\begin{array}{c}\text { Volume of } \\
\text { Gas (mL) }\end{array}$ & $\begin{array}{c}\text { Mass of } \\
\mathrm{PH}_{3}(\mathrm{~g})\end{array}$ & $\begin{array}{c}\text { Yield } \\
(\%)\end{array}$ & $\begin{array}{c}\text { Time } \\
(\mathrm{min})\end{array}$ & $\begin{array}{c}\text { Volume of } \\
\text { Gas (mL) }\end{array}$ & $\begin{array}{c}\text { Mass of } \\
\mathrm{PH}_{3}(\mathrm{~g})\end{array}$ & $\begin{array}{c}\text { Yield } \\
(\%)\end{array}$ \\
\hline 0.33 & 4.8 & 0.0102 & 15.0 & 1 & 1.5 & 0.0032 & 4.7 \\
\hline 0.67 & 5.5 & 0.0117 & 17.2 & 2 & 2.3 & 0.0049 & 7.2 \\
\hline 1 & 6.2 & 0.0132 & 19.4 & 3 & 2.9 & 0.0062 & 9.1 \\
\hline 2 & 6.6 & 0.0140 & 20.6 & 5 & 4.0 & 0.0085 & 12.5 \\
\hline 3 & 6.7 & 0.0142 & 20.9 & 7 & 5.0 & 0.0106 & 15.6 \\
\hline 5 & 6.9 & 0.0146 & 21.6 & 10 & 6.2 & 0.0132 & 19.4 \\
\hline 10 & 6.9 & 0.0146 & 21.6 & 15 & 7.4 & 0.0157 & 23.1 \\
\hline & & & & 20 & 8.1 & 0.0172 & 25.3 \\
\hline & & & & 30 & 9.5 & 0.0202 & 29.7 \\
\hline & & & & 40 & 10.8 & 0.0229 & 33.8 \\
\hline & & & & 50 & 12.6 & 0.0267 & 39.4 \\
\hline & & & & 60 & 13.8 & 0.0293 & 43.1 \\
\hline & & & & 90 & 14.6 & 0.0310 & 45.6 \\
\hline & & & & 120 & 15.0 & 0.0318 & 46.9 \\
\hline & & & & 150 & 15.1 & 0.0320 & 47.2 \\
\hline
\end{tabular}

Comments: The reaction was incomplete in all four experiments, although in 2003 a different sample of $\mathrm{Ca}_{3} \mathrm{P}_{2}$ was used, and the yields of $\mathrm{PH}_{3}$ were higher than in 1999. In method A, the reaction mixture thickened and became difficult to stir. In method B, larger amounts of gas were evolved than in method $\mathrm{A}$. The solubility of $\mathrm{PH}_{3}$ is negligible in both water and octane. Dissolution in the excess water did not therefore remove any gas from the reaction system. Chemical events in the mixture were almost certainly more complex than suggested by the single equation given above. For example, gaseous diphosphine $\left[\mathrm{P}_{2} \mathrm{H}_{4}(\mathrm{~g})\right]$ might have formed. The extent of such a concurrent reaction cannot be determined in this experiment, which measures only the total volume of evolved gases. The yields and masses in the table are based on the assumption that the gaseous product was pure $\mathrm{PH}_{3}$. 


\section{UN 1384 - Sodium Dithionite, $\mathrm{Na}_{2} \mathrm{~S}_{2} \mathrm{O}_{4}$}

Sodium dithionite, also called sodium hydrosulfite, is a solid with a relative molar mass of 174.11. In method A, $1.00 \mathrm{mmol}$ of it was mixed with $1.00 \mathrm{mmol}$ of water. In method B, $1.00 \mathrm{mmol}$ of it was mixed with $5.00 \mathrm{mmol}$ of water.

Comments: The purpose was to check on the evolution of $\mathrm{H}_{2} \mathrm{~S}(\mathrm{~g})$ and/or $\mathrm{SO}_{2}(\mathrm{~g})$ by means of a disproportionation reaction. No measurable amounts of gas were evolved within 30 min using method A or within 300 min using method B. In separate test-tube experiments, the presence of both $\mathrm{H}_{2} \mathrm{~S}(\mathrm{~g})$ and $\mathrm{SO}_{2}(\mathrm{~g})$ was recognized by their odors. The amounts of these acidic gases were sufficient to change the color of moistened $\mathrm{pH}$ paper.

\section{UN 1412 - Lithium Amide, LiNH2}

Lithium amide is a solid with a relative molar mass of 22.96. In method A, $1.00 \mathrm{mmol}$ of water was added to $1.00 \mathrm{mmol}$ of it. In method B, $1.00 \mathrm{mmol}$ of it was mixed with $5.00 \mathrm{mmol}$ of water. Reaction according to the equation

$$
\mathrm{LiNH}_{2}+\mathrm{H}_{2} \mathrm{O} \longrightarrow \mathrm{NH}_{3}+\mathrm{LiOH}
$$

was taken as the source of $\mathrm{NH}_{3}(\mathrm{~g})$ for computations of mass and yield. The theoretical yield of $\mathrm{NH}_{3}$ (g) was $1.00 \mathrm{mmol}$, which corresponded to $16.0 \mathrm{~mL}$ of gas in the calibrated experimental apparatus.

\section{Experimental Data for Lithium Amide}

\begin{tabular}{|c|c|c|c|c|c|c|c|}
\hline \multicolumn{4}{|c|}{ Method A } & \multicolumn{4}{c|}{ Method B } \\
\hline $\begin{array}{c}\text { Time } \\
(\mathrm{min})\end{array}$ & $\begin{array}{c}\text { Volume of } \\
\text { Gas (mL) }\end{array}$ & $\begin{array}{c}\text { Mass of } \\
\mathrm{NH}_{3}(\mathrm{~g})\end{array}$ & $\begin{array}{c}\text { Yield } \\
(\%)\end{array}$ & $\begin{array}{c}\text { Time } \\
(\mathrm{min})\end{array}$ & $\begin{array}{c}\text { Volume of } \\
\text { Gas (mL) }\end{array}$ & $\begin{array}{c}\text { Mass of } \\
\mathrm{NH}_{3}(\mathrm{~g})\end{array}$ & $\begin{array}{c}\text { Yield } \\
(\%)\end{array}$ \\
\hline 0.33 & 2.4 & 0.0025 & 14.5 & 0.33 & 3.1 & 0.0032 & 18.8 \\
\hline 0.67 & 3.0 & 0.0031 & 18.2 & 0.5 & 3.6 & 0.0037 & 21.8 \\
\hline 1 & 3.2 & 0.0033 & 19.4 & 1 & 3.1 & 0.0032 & 18.8 \\
\hline 2 & 3.5 & 0.0036 & 21.2 & 2 & 2.7 & 0.0028 & 16.4 \\
\hline 3 & 3.7 & 0.0038 & 22.4 & 3 & 2.6 & 0.0027 & 15.8 \\
\hline 5 & 3.9 & 0.0040 & 23.6 & 5 & 2.4 & 0.0025 & 14.5 \\
\hline 7 & 4.1 & 0.0042 & 24.8 & 7 & 2.3 & 0.0024 & 13.9 \\
\hline 10 & 4.3 & 0.0044 & 26.1 & 10 & 2.2 & 0.0023 & 13.3 \\
\hline 15 & 4.6 & 0.0047 & 27.9 & 15 & 2.1 & 0.0022 & 12.7 \\
\hline 20 & 4.7 & 0.0049 & 28.5 & 20 & 2.1 & 0.0022 & 12.7 \\
\hline 30 & 4.8 & 0.0050 & 29.1 & & & & \\
\hline 40 & 4.8 & 0.0050 & 29.1 & & & & \\
\hline
\end{tabular}


Comments: In method A, the reaction was rapid at first but ultimately produced less than one third of the theoretical amount of $\mathrm{NH}_{3}(\mathrm{~g})$. In method $\mathrm{B}$, the reaction was apparently less productive of $\mathrm{NH}_{3}(\mathrm{~g})$ but the maximum on the yield curve for $\mathrm{NH}_{3}(\mathrm{~g})$ indicated that some $\mathrm{NH}_{3}(\mathrm{~g})$ dissolved in the excess water.

\section{UN 1433 - Stannic Phosphide, $\mathrm{Sn}_{4} \mathbf{P}_{3}$}

Stannic phosphide, which has a relative molar mass of 567.70, is metallic. In method A, $0.567 \mathrm{~g}(1.00 \mathrm{mmol})$ of it was mixed with $0.144 \mathrm{~g}(8.0 \mathrm{mmol})$ of water. In method $\mathrm{B}, 1.00 \mathrm{mmol}$ of it was mixed with $0.720 \mathrm{~g}(40.0 \mathrm{mmol})$ of water.

Comments: The purpose of the experiment was to check on the possible evolution of phosphine $\left(\mathrm{PH}_{3}\right)$. No measurable amount of gas was evolved within 20 min using either method. Also, no odor of $\mathrm{PH}_{3}$ was detected.

\section{UN 1541 - Acetone Cyanohydrin, Stabilized, $\left(\mathrm{CH}_{3}\right)_{2} \mathrm{C}(\mathrm{OH}) \mathrm{CN}$}

Acetone cyanohydrin is a liquid with a relative molar mass of 85.11 and a density of $0.932 \mathrm{~g} / \mathrm{cm}^{3}$. In method A, $1.00 \mathrm{mmol}$ of water was added to $1.00 \mathrm{mmol}$ of it. In method B, $1.00 \mathrm{mmol}$ of it was added to $5.00 \mathrm{mmol}$ of water. The equilibrium reaction

$$
\left(\mathrm{CH}_{3}\right)_{2} \mathrm{C}(\mathrm{OH}) \mathrm{CN} \longrightarrow \mathrm{HCN}+\left(\mathrm{CH}_{3}\right)_{2} \mathrm{CO}
$$

was considered as a source of evolved HCN. Generation of $1.00 \mathrm{mmol}$ of gas corresponded to an increase in volume of $16.0 \mathrm{~mL}$ in the calibrated apparatus.

Comments: No measurable amount of gas was evolved within 30 min using either method. Contact of this substance with acidic aqueous solutions is known to cause evolution of $\mathrm{HCN}(\mathrm{g})$.

\section{UN 1714 - Zinc Phosphide, $\mathbf{Z n}_{3} \mathbf{P}_{2}$}

Zinc phosphide is a solid with a relative molar mass of 258.11. In method A, $0.258 \mathrm{~g}$ $(1.00 \mathrm{mmol})$ of it was mixed with $6.00 \mathrm{mmol}$ of water. In method B, $1.00 \mathrm{mmol}$ of it was mixed with $30.0 \mathrm{mmol}$ of water. The equation

$$
\mathrm{Zn}_{3} \mathrm{P}_{2}+6 \mathrm{H}_{2} \mathrm{O} \longrightarrow 2 \mathrm{PH}_{3}+3 \mathrm{Zn}(\mathrm{OH})_{2}
$$

was taken to represent the evolution of gas. The theoretical yield of $\mathrm{PH}_{3}(\mathrm{~g})(2.00 \mathrm{mmol})$ corresponded to $32.0 \mathrm{~mL}$ of gas in the calibrated experimental apparatus. 
Comments: No measurable amount of gas was generated within 30 min using method A and within 20 minutes using method B. Also, the odor of $\mathrm{PH}_{3}$ was not detected.

\section{UN 1716 - Acetyl Bromide, $\mathrm{CH}_{3} \mathrm{COBr}$}

Acetyl bromide is a colorless liquid with a relative molar mass of 122.95 and a roomtemperature density of $1.663 \mathrm{~g} / \mathrm{cm}^{3}$. In method A, $1.00 \mathrm{mmol}$ of water was added to $1.00 \mathrm{mmol}$ of it. In method B, $1.00 \mathrm{mmol}$ of it was mixed with $5.00 \mathrm{mmol}$ of water. Reaction according to the equation

$$
\mathrm{CH}_{3} \mathrm{COBr}+\mathrm{H}_{2} \mathrm{O} \longrightarrow \mathrm{HBr}+\mathrm{CH}_{3} \mathrm{COOH}
$$

was taken as the source of $\operatorname{HBr}(\mathrm{g})$ for computations of mass and yield. The theoretical yield of $\mathrm{HBr}(\mathrm{g})$ was $1.00 \mathrm{mmol}$, which corresponded to $16.0 \mathrm{~mL}$ of gas in the calibrated experimental apparatus.

2003 Experimental Data for Acetyl Bromide

\begin{tabular}{|c|c|c|c|c|c|c|c|}
\hline \multicolumn{4}{|c|}{ Method A } & \multicolumn{4}{c|}{ Method B } \\
\hline $\begin{array}{c}\text { Time } \\
(\mathrm{min})\end{array}$ & $\begin{array}{c}\text { Volume of } \\
\text { Gas (mL) }\end{array}$ & $\begin{array}{c}\text { Mass of } \\
\mathrm{HBr}(\mathrm{g})\end{array}$ & $\begin{array}{c}\text { Yield } \\
(\%)\end{array}$ & $\begin{array}{c}\text { Time } \\
(\mathrm{min})\end{array}$ & $\begin{array}{c}\text { Volume of } \\
\text { Gas }(\mathrm{mL})\end{array}$ & $\begin{array}{c}\text { Mass of } \\
\mathrm{HBr}(\mathrm{g})\end{array}$ & $\begin{array}{c}\text { Yield } \\
(\%)\end{array}$ \\
\hline 0.25 & 11.1 & 0.056 & 69.4 & 0.25 & 5.3 & 0.0268 & 33.1 \\
\hline 0.5 & 15.5 & 0.078 & 96.9 & 0.5 & 1.2 & 0.0061 & 7.5 \\
\hline 1 & 15.7 & 0.079 & 98.1 & 1 & 0.5 & 0.0025 & 3.1 \\
\hline 2 & 16.2 & 0.082 & 101.3 & 2 & 0.2 & 0.0010 & 1.3 \\
\hline 5 & 16.2 & 0.082 & 101.3 & 3 & 0.1 & 0.0005 & 0.6 \\
\hline
\end{tabular}

Comments: In method $\mathrm{A}$, the theoretical amount of $\mathrm{HBr}(\mathrm{g})$ was evolved rapidly. In method $\mathrm{B}$, the reaction was faster than in method $\mathrm{A} . \mathrm{HBr}(\mathrm{g})$ dissolved in the excess water throughout the gas-producing reaction. No maximum was found on the $\mathrm{HBr}(\mathrm{g})$ yield curve.

\section{UN 1717 - Acetyl Chloride, $\mathrm{CH}_{3} \mathrm{COCl}$}

Acetyl chloride is a colorless liquid with a relative molar mass of 78.50 and a roomtemperature density of $1.104 \mathrm{~g} / \mathrm{cm}^{3}$. In method A, $1.00 \mathrm{mmol}$ of water was added to $1.00 \mathrm{mmol}$ of it. In method B, $1.00 \mathrm{mmol}$ of it was mixed with $5.00 \mathrm{mmol}$ of water. Reaction according to the equation

$$
\mathrm{CH}_{3} \mathrm{COCl}+\mathrm{H}_{2} \mathrm{O} \longrightarrow \mathrm{HCl}+\mathrm{CH}_{3} \mathrm{COOH}
$$


was taken as the source of $\mathrm{HCl}(\mathrm{g})$ for computations of mass and yield. The theoretical yield of $\mathrm{HCl}(\mathrm{g})$ was $1.00 \mathrm{mmol}$, which corresponded to $16.0 \mathrm{~mL}$ of gas in the calibrated experimental apparatus.

\section{Experimental Data for Acetyl Chloride}

\begin{tabular}{|c|c|c|c|c|c|c|c|}
\hline \multicolumn{4}{|c|}{ Method A } & \multicolumn{4}{c|}{ Method B } \\
\hline $\begin{array}{c}\text { Time } \\
(\mathrm{min})\end{array}$ & $\begin{array}{c}\text { Volume of } \\
\text { Gas (mL) }\end{array}$ & $\begin{array}{c}\text { Mass of } \\
\mathrm{HCl}(\mathrm{g})\end{array}$ & $\begin{array}{c}\text { Yield } \\
(\%)\end{array}$ & $\begin{array}{c}\text { Time } \\
(\mathrm{min})\end{array}$ & $\begin{array}{c}\text { Volume of } \\
\text { Gas (mL) }\end{array}$ & $\begin{array}{c}\text { Mass of } \\
\mathrm{HCl}(\mathrm{g})\end{array}$ & $\begin{array}{c}\text { Yield } \\
(\%)\end{array}$ \\
\hline 0.33 & 10.5 & 0.0239 & 65.6 & 0.33 & 6.0 & 0.0137 & 37.5 \\
\hline 0.67 & 13.5 & 0.0308 & 84.4 & 0.67 & 4.5 & 0.010 & 28.1 \\
\hline 1 & 14.7 & 0.0335 & 91.9 & 1 & 3.2 & 0.0073 & 20.0 \\
\hline 2 & 15.7 & 0.0358 & 98.1 & 1.5 & 2.3 & 0.0052 & 14.4 \\
\hline 3 & 15.9 & 0.0362 & 99.4 & 2 & 1.6 & 0.0036 & 10.0 \\
\hline 5 & 15.9 & 0.0362 & 99.4 & 3 & 1.0 & 0.0023 & 6.3 \\
\hline & & & & 5 & 0.2 & 0.0005 & 1.3 \\
\hline & & & & 10 & 0.0 & 0.0000 & 0.0 \\
\hline
\end{tabular}

Comments: In method $\mathrm{A}$, the theoretical amount of $\mathrm{HCl}(\mathrm{g})$ evolved rapidly. In method $\mathrm{B}$, the formation of $\mathrm{HCl}(\mathrm{g})$ was also rapid, but it dissolved in the water/acetic acid mixture almost as fast as it formed. All of the $\mathrm{HCl}(\mathrm{g})$ ultimately dissolved. No maximum was observed on the $\mathrm{HCl}(\mathrm{g})$ yield curve.

\section{UN 1724 - Allyltrichlorosilane, $\mathrm{CH}_{2}=\mathrm{CHCH}_{2} \mathrm{SiCl}_{3}$}

Allyltrichlorosilane is a liquid with a relative molar mass of 175.52 and a roomtemperature density of $1.211 \mathrm{~g} / \mathrm{cm}^{3}$. In method A, $1.00 \mathrm{mmol}$ of it was mixed with $1.50 \mathrm{mmol}$ of water. In method B, $1.00 \mathrm{mmol}$ of it was mixed with $7.50 \mathrm{mmol}$ of water. Reaction according to the equation

$$
\mathrm{CH}_{2} \mathrm{CHCH}_{2} \mathrm{SiCl}_{3}+3 / 2 \mathrm{H}_{2} \mathrm{O} \longrightarrow 3 \mathrm{HCl}+\left[\mathrm{CH}_{2} \mathrm{CHCH}_{2} \mathrm{SiO}_{3 / 2}\right]_{\mathrm{n}} \text { polymer }
$$

was taken as the source of $\mathrm{HCl}(\mathrm{g})$. The theoretical yield of $\mathrm{HCl}(\mathrm{g})$ was $3.00 \mathrm{mmol}$, which corresponded to $48.0 \mathrm{~mL}$ of gas in the calibrated experimental apparatus. 


\begin{tabular}{|c|c|c|c|c|c|c|c|}
\hline \multicolumn{8}{|c|}{2003 Experimental Data for Allyltrichlorosilane } \\
\hline \multicolumn{4}{|c|}{ Method A } & \multicolumn{4}{|c|}{ Method B } \\
\hline $\begin{array}{l}\text { Time } \\
\text { (min) }\end{array}$ & $\begin{array}{l}\text { Volume of } \\
\text { Gas (mL) }\end{array}$ & $\begin{array}{l}\text { Mass of } \\
\mathrm{HCl}(\mathrm{g})\end{array}$ & $\begin{array}{l}\text { Yield } \\
(\%)\end{array}$ & $\begin{array}{l}\text { Time } \\
(\min )\end{array}$ & $\begin{array}{l}\text { Volume of } \\
\text { Gas (mL) }\end{array}$ & $\begin{array}{l}\text { Mass of } \\
\mathrm{HCl}(\mathrm{g})\end{array}$ & $\begin{array}{c}\text { Yield } \\
(\%)\end{array}$ \\
\hline 0.33 & 2.7 & 0.0062 & 5.6 & 0.5 & 15.0 & 0.034 & 31.3 \\
\hline 0.67 & 5.1 & 0.0116 & 10.6 & 1 & 23.8 & 0.054 & 49.6 \\
\hline 1 & 8.4 & 0.0191 & 17.5 & 2 & 23.2 & 0.053 & 48.3 \\
\hline 1.5 & 13.2 & 0.0301 & 27.5 & 3 & 21.6 & 0.049 & 45.0 \\
\hline 2 & 16.7 & 0.038 & 34.8 & 4 & 20.2 & 0.046 & 42.1 \\
\hline 2.5 & 19.2 & 0.044 & 40.0 & 5 & 19.1 & 0.044 & 39.8 \\
\hline 3 & 20.9 & 0.048 & 43.5 & 6 & 18.5 & 0.042 & 38.5 \\
\hline 4 & 24.6 & 0.056 & 51.3 & 8 & 17.5 & 0.040 & 36.5 \\
\hline 5 & 28.8 & 0.066 & 60.0 & 10 & 16.5 & 0.038 & 34.4 \\
\hline 6 & 41.4 & 0.094 & 86.3 & 12 & 15.9 & 0.036 & 33.1 \\
\hline 7 & 43.4 & 0.099 & 90.4 & 15 & 15.3 & 0.035 & 31.9 \\
\hline 8 & 43.5 & 0.099 & 90.6 & 20 & 15.0 & 0.034 & 31.3 \\
\hline 10 & 43.6 & 0.099 & 90.8 & 30 & 14.9 & 0.034 & 31.0 \\
\hline
\end{tabular}

Comments: With both methods, the reaction rapidly formed a viscous polymeric siloxane. In method $\mathrm{B}$, the yield of gaseous $\mathrm{HCl}$ reached a maximum in less than $1 \mathrm{~min}$. This was followed by the absorption of $\mathrm{HCl}(\mathrm{g})$ by the excess water.

\section{UN 1725 - Aluminum Bromide (anhydrous), AlBr3}

Aluminum bromide, a solid, has a relative molar mass of 266.69. In method A, $1.00 \mathrm{mmol}$ of it was mixed with $1.00 \mathrm{mmol}$ of water. In method B, $1.00 \mathrm{mmol}$ was mixed with $5.00 \mathrm{mmol}$ of water. No equation was proposed for a reaction generating a TIH gas beyond the following:

$$
\mathrm{AlBr}_{3}+\mathrm{n} \mathrm{H}_{2} \mathrm{O} \longrightarrow \mathrm{AlBr}_{3} \bullet\left(\mathrm{H}_{2} \mathrm{O}\right)_{\mathrm{n}} \text { (hydration) }
$$

1999 Experimental Results for Aluminum Bromide (anhydrous)

\begin{tabular}{|c|c|c|c|c|c|c|c|}
\hline \multicolumn{4}{|c|}{ Method A } & \multicolumn{5}{c|}{ Method B } \\
\hline $\begin{array}{c}\text { Time } \\
(\mathrm{min})\end{array}$ & $\begin{array}{c}\text { Volume of } \\
\text { Gas (mL) }\end{array}$ & $\begin{array}{c}\text { Mass of } \\
\mathrm{HBr}(\mathrm{g})\end{array}$ & $\begin{array}{c}\text { Percent } \\
\text { Yield }\end{array}$ & $\begin{array}{c}\text { Time } \\
(\mathrm{min})\end{array}$ & $\begin{array}{c}\text { Volume of } \\
\text { Gas (mL) }\end{array}$ & $\begin{array}{c}\text { Mass of } \\
\mathrm{HBr}(\mathrm{g})\end{array}$ & $\begin{array}{c}\text { Percent } \\
\text { Yield }\end{array}$ \\
\hline 1 & 1 & - & - & 1 & 1 & - & - \\
\hline 5 & 1 & - & - & 5 & 1 & - & - \\
\hline 10 & 0.5 & - & - & 10 & 0.5 & - & - \\
\hline 20 & 0.5 & - & - & 20 & 0.5 & - & - \\
\hline
\end{tabular}


Comments: The hydration reaction was rapid and quite exothermic. The observed small changes in volume were attributed solely to this heating. The production of an acidic mist by the intense heating of a restricted quantity of water cannot be ruled out in a spill of a large amount of aluminum bromide into a restricted supply of water.

\section{UN 1726 - Aluminum Chloride (anhydrous), $\mathrm{AlCl}_{3}$}

Aluminum chloride, a solid, has a relative molar mass of 133.34. In method A, $1.00 \mathrm{mmol}$ of it was mixed with $1.00 \mathrm{mmol}$ of water. In method B, $1.00 \mathrm{mmol}$ was mixed with $5.00 \mathrm{mmol}$ of water. No equation was proposed for a reaction generating a TIH gas beyond the following:

$$
\mathrm{AlCl}_{3}+\mathrm{n} \mathrm{H}_{2} \mathrm{O} \longrightarrow \mathrm{AlCl}_{3} \bullet\left(\mathrm{H}_{2} \mathrm{O}\right)_{\mathrm{n}} \text { (hydration) }
$$

1999 Experimental Results for Aluminum Chloride (anhydrous)

\begin{tabular}{|c|c|c|c|c|c|c|c|}
\hline \multicolumn{4}{|c|}{ Method A } & \multicolumn{5}{c|}{ Method B } \\
\hline $\begin{array}{c}\text { Time } \\
(\mathrm{min})\end{array}$ & $\begin{array}{c}\text { Volume of } \\
\text { Gas (mL) }\end{array}$ & $\begin{array}{c}\text { Mass of } \\
\mathrm{HCl}(\mathrm{g})\end{array}$ & $\begin{array}{c}\text { Yield } \\
(\%)\end{array}$ & $\begin{array}{c}\text { Time } \\
(\mathrm{min})\end{array}$ & $\begin{array}{c}\text { Volume of } \\
\text { Gas (mL) }\end{array}$ & $\begin{array}{c}\text { Mass of } \\
\mathrm{HCl}(\mathrm{g})\end{array}$ & $\begin{array}{c}\text { Yield } \\
(\%)\end{array}$ \\
\hline 1 & 1 & - & - & 1 & 1 & - & - \\
\hline 5 & 1 & - & - & 5 & 1 & - & - \\
\hline 10 & 0.5 & - & - & 10 & 0.5 & - & - \\
\hline 20 & 0.5 & - & - & 20 & 0.5 & - & - \\
\hline
\end{tabular}

Comments: The hydration reaction was rapid and quite exothermic. The observed small change in volume was attributed solely to this heating. The production of an acidic mist by intense heating of a restricted quantity of water should not be ruled out as the result of a spill of a large quantity of aluminum chloride into a restricted supply of water.

\section{UN 1728 - Amyltrichlorosilane, $\mathrm{C}_{5} \mathrm{H}_{11} \mathrm{SiCl}_{3}$}

Amyltrichlorosilane, also known as (n-pentyl)trichlorosilane, is a liquid with a relative molar mass of 205.59 and a room-temperature density of $1.142 \mathrm{~g} / \mathrm{cm}^{3}$. In method A, $1.00 \mathrm{mmol}$ of it was mixed with $1.50 \mathrm{mmol}$ of water. In method B, $1.00 \mathrm{mmol}$ of it was mixed with $7.50 \mathrm{mmol}$ of water. The equation

$$
\mathrm{C}_{5} \mathrm{H}_{11} \mathrm{SiCl}_{3}+3 / 2 \mathrm{H}_{2} \mathrm{O} \longrightarrow 3 \mathrm{HCl}+\left[\mathrm{C}_{5} \mathrm{H}_{11} \mathrm{SiO}_{3 / 2}\right]_{\mathrm{n}} \text { polymer }
$$

was taken to represent the evolution of $\mathrm{HCl}(\mathrm{g})$. The theoretical yield of $\mathrm{HCl}(\mathrm{g})$ was $3.00 \mathrm{mmol}$, which corresponded to $48.0 \mathrm{~mL}$ of gas in the calibrated experimental apparatus. 


\begin{tabular}{|c|c|c|c|c|c|c|c|}
\hline \multicolumn{8}{|c|}{2003 Experimental Data for Amyltrichlorosilane } \\
\hline \multicolumn{4}{|c|}{ Method A } & \multicolumn{4}{|c|}{ Method B } \\
\hline $\begin{array}{l}\text { Time } \\
\text { (min) }\end{array}$ & $\begin{array}{l}\text { Volume of } \\
\text { Gas (mL) }\end{array}$ & $\begin{array}{l}\text { Mass of } \\
\mathrm{HCl}(\mathrm{g})\end{array}$ & $\begin{array}{c}\text { Yield } \\
(\%)\end{array}$ & $\begin{array}{l}\text { Time } \\
(\mathrm{min})\end{array}$ & $\begin{array}{l}\text { Volume of } \\
\text { Gas (mL) }\end{array}$ & $\begin{array}{l}\text { Mass of } \\
\mathrm{HCl}(\mathrm{g})\end{array}$ & $\begin{array}{c}\text { Yield } \\
(\%)\end{array}$ \\
\hline 1 & 0.2 & 0.0005 & 0.4 & 1 & 0.3 & 0.0007 & 0.6 \\
\hline 3 & 0.3 & 0.0007 & 0.6 & 3 & 0.7 & 0.0016 & 1.5 \\
\hline 5 & 0.4 & 0.0009 & 0.8 & 5 & 1.7 & 0.0039 & 3.5 \\
\hline 10 & 0.9 & 0.0021 & 1.9 & 10 & 3.5 & 0.0080 & 7.3 \\
\hline 15 & 1.2 & 0.0027 & 2.5 & 15 & 5.7 & 0.0130 & 11.9 \\
\hline 20 & 2.0 & 0.0046 & 4.2 & 20 & 6.6 & 0.0150 & 13.8 \\
\hline 30 & 3.0 & 0.0068 & 6.3 & 25 & 7.3 & 0.0166 & 15.2 \\
\hline 40 & 5.2 & 0.0119 & 10.8 & 30 & 8.1 & 0.0185 & 16.9 \\
\hline 50 & 5.9 & 0.0134 & 12.3 & 35 & 8.8 & 0.0201 & 18.3 \\
\hline 60 & 6.9 & 0.0157 & 14.4 & 40 & 9.3 & 0.0212 & 19.4 \\
\hline 75 & 7.8 & 0.0178 & 16.3 & 50 & 10.0 & 0.0228 & 20.8 \\
\hline 90 & 8.7 & 0.0198 & 18.1 & 60 & 10.3 & 0.0235 & 21.5 \\
\hline 105 & 9.6 & 0.0219 & 20.0 & 90 & 10.4 & 0.0237 & 21.7 \\
\hline 120 & 10.5 & 0.0239 & 21.9 & & & & \\
\hline 135 & 11.3 & 0.0258 & 23.5 & & & & \\
\hline 150 & 12.8 & 0.0292 & 26.7 & & & & \\
\hline 165 & 14.7 & 0.0335 & 30.6 & & & & \\
\hline 180 & 14.8 & 0.0337 & 30.8 & & & & \\
\hline
\end{tabular}

Comments: In method A, the reaction slowly formed a very viscous liquid polysiloxane. This product apparently sequestered a considerable amount of water because less than one-third of the stoichiometric amount of $\mathrm{HCl}(\mathrm{g})$ evolved. In method $\mathrm{B}$, the viscous liquid by-product again was formed. The reaction was slow enough that the $\mathrm{HCl}(\mathrm{g})$ dissolved without a maximum on the yield curve.

\section{UN 1732 - Antimony Pentafluoride, $\mathrm{SbF}_{5}$}

Antimony pentafluoride is a liquid with a relative molar mass of 216.74 and a roomtemperature density of $2.99 \mathrm{~g} / \mathrm{cm}^{3}$. In method $\mathrm{A}, 1.00 \mathrm{mmol}$ of it was mixed with $5.00 \mathrm{mmol}$ of water. In method B, $1.00 \mathrm{mmol}$ of it was mixed with $25.0 \mathrm{mmol}$ of water. The equation

$$
2 \mathrm{SbF}_{5}+5 \mathrm{H}_{2} \mathrm{O} \longrightarrow 10 \mathrm{HF}+\mathrm{Sb}_{2} \mathrm{O}_{5}
$$

was taken to represent the evolution of HF. The theoretical yield of $\mathrm{HF}(\mathrm{g})$ was $10.0 \mathrm{mmol}$, which corresponded to $160 \mathrm{~mL}$ of gas in the calibrated experimental apparatus.

Comments: No evolution of gas was observed with either method A or method B, even after 20-30 min. This experiment was the first attempt to measure the evolution of $\mathrm{HF}(\mathrm{g})$. Both 
$\mathrm{SbF}_{5}$ and $\mathrm{HF}$ are difficult to handle. This result cannot be regarded as conclusive because of the experimental difficulties.

\section{UN 1736 - Benzoyl Chloride, $\mathrm{C}_{6} \mathrm{H}_{5} \mathrm{COCl}$}

Benzoyl chloride is a liquid with relative molar mass of 140.57 and a room-temperature density of $1.211 \mathrm{~g} / \mathrm{cm}^{3}$. In method A, $1.00 \mathrm{mmol}$ of it was mixed with $1.00 \mathrm{mmol}$ of water. In method B, $1.00 \mathrm{mmol}$ of it was mixed with $5.00 \mathrm{mmol}$ of water. Reaction according to the equation

$$
\mathrm{C}_{6} \mathrm{H}_{5} \mathrm{COCl}+\mathrm{H}_{2} \mathrm{O} \longrightarrow \mathrm{HCl}+\mathrm{C}_{6} \mathrm{H}_{5} \mathrm{COOH}
$$

was taken as the source of $\mathrm{HCl}(\mathrm{g})$. The theoretical yield of $\mathrm{HCl}(\mathrm{g})$ was $1.00 \mathrm{mmol}$, which corresponded to $16.0 \mathrm{~mL}$ of gas in the calibrated experimental apparatus.

Comments: No measurable volume change was observed within 20 min using either method. Thus, within the observed time period the rate of hydrolysis of benzoyl chloride is negligible.

\section{UN 1747 - Butyltrichlorosilane, $\mathrm{C}_{4} \mathrm{H}_{9} \mathrm{SiCl}_{3}$}

Butyltrichlorosilane is a liquid with a relative molar mass of 191.56 and a roomtemperature density of $1.160 \mathrm{~g} / \mathrm{cm}^{3}$. In method A, $1.00 \mathrm{mmol}$ of it was mixed with $1.50 \mathrm{mmol}$ of water. In method B, $1.00 \mathrm{mmol}$ of it was mixed with $7.50 \mathrm{mmol}$ of water. The equation

$$
\mathrm{C}_{4} \mathrm{H}_{9} \mathrm{SiCl}_{3}+3 / 2 \mathrm{H}_{2} \mathrm{O} \longrightarrow 3 \mathrm{HCl}+\left[\mathrm{C}_{4} \mathrm{H}_{9} \mathrm{SiO}_{3 / 2}\right]_{\mathrm{n}} \text { polymer }
$$

was taken to represent the evolution of $\mathrm{HCl}(\mathrm{g})$. The theoretical yield of $\mathrm{HCl}(\mathrm{g})$ was $3.00 \mathrm{mmol}$, which corresponded to $48.0 \mathrm{~mL}$ of gas in the calibrated experimental apparatus. 


\begin{tabular}{|c|c|c|c|c|c|c|c|}
\hline \multicolumn{8}{|c|}{2003 Experimental Data for Butyltrichlorosilane } \\
\hline \multicolumn{4}{|c|}{ Method A } & \multicolumn{4}{|c|}{ Method B } \\
\hline $\begin{array}{l}\text { Time } \\
\text { (min) }\end{array}$ & $\begin{array}{l}\text { Volume of } \\
\text { Gas (mL) }\end{array}$ & $\begin{array}{l}\text { Mass of } \\
\mathrm{HCl}(\mathrm{g})\end{array}$ & $\begin{array}{c}\text { Yield } \\
(\%)\end{array}$ & $\begin{array}{l}\text { Time } \\
\text { (min) }\end{array}$ & $\begin{array}{l}\text { Volume of } \\
\text { Gas (mL) }\end{array}$ & $\begin{array}{l}\text { Mass of } \\
\mathrm{HCl}(\mathrm{g})\end{array}$ & $\begin{array}{c}\text { Yield } \\
(\%)\end{array}$ \\
\hline 1 & 0.2 & 0.0005 & 0.4 & 1 & 0.3 & 0.0007 & 0.6 \\
\hline 2 & 0.5 & 0.0011 & 1.0 & 2 & 0.5 & 0.0011 & 1.0 \\
\hline 3 & 0.7 & 0.0016 & 1.5 & 3 & 0.7 & 0.0016 & 1.5 \\
\hline 5 & 1.0 & 0.0023 & 2.1 & 5 & 1.1 & 0.0025 & 2.3 \\
\hline 7 & 1.5 & 0.0034 & 3.1 & 7 & 1.9 & 0.0043 & 4.0 \\
\hline 10 & 2.3 & 0.0052 & 4.8 & 10 & 3.6 & 0.0082 & 7.5 \\
\hline 15 & 3.6 & 0.0082 & 7.5 & 15 & 6.7 & 0.0153 & 14.0 \\
\hline 20 & 4.9 & 0.0112 & 10.2 & 20 & 8.8 & 0.0201 & 18.3 \\
\hline 25 & 6.1 & 0.0139 & 12.7 & 25 & 10.5 & 0.0239 & 21.9 \\
\hline 30 & 7.2 & 0.0164 & 15.0 & 30 & 11.5 & 0.0262 & 24.0 \\
\hline 35 & 8.0 & 0.0182 & 16.7 & 40 & 12.0 & 0.0274 & 25.0 \\
\hline 40 & 8.8 & 0.0201 & 18.3 & 50 & 12.1 & 0.0276 & 25.2 \\
\hline 50 & 10.0 & 0.0228 & 20.8 & 60 & 12.1 & 0.0276 & 25.2 \\
\hline 60 & 11.2 & 0.0255 & 23.3 & & & & \\
\hline 75 & 12.3 & 0.0280 & 25.6 & & & & \\
\hline 90 & 14.2 & 0.032 & 29.6 & & & & \\
\hline 105 & 16.9 & 0.039 & 35.2 & & & & \\
\hline 120 & 18.4 & 0.042 & 38.3 & & & & \\
\hline 150 & 18.5 & 0.042 & 38.5 & & & & \\
\hline
\end{tabular}

Comments: In method A, the reaction slowly formed a very viscous liquid by-product that apparently sequestered a considerable amount of water. In method B, the reaction also formed the viscous by-product. The reaction was slow enough that the $\mathrm{HCl}(\mathrm{g})$ dissolved without a maximum on the yield curve.

\section{UN 1748 - Calcium Hypochlorite, $\mathrm{Ca}(\mathrm{OCl})_{2}$}

Calcium hypochlorite, a solid, has a relative molar mass of 142.98. In method A, $1.00 \mathrm{mmol}$ of the substance was mixed with $1.00 \mathrm{mmol}$ of water. In method B, $1.00 \mathrm{mmol}$ was mixed with $5.00 \mathrm{mmol}$ of water. No equation was proposed for a reaction generating a TIH gas beyond the following

$$
\mathrm{Ca}(\mathrm{OCl})_{2}+\mathrm{H}_{2} \mathrm{O} \longrightarrow \mathrm{Cl}_{2}+\text { other products }
$$


1999 Experimental Results for Calcium Hypochlorite

\begin{tabular}{|c|c|c|c|c|c|c|c|}
\hline \multicolumn{4}{|c|}{ Method A } & \multicolumn{4}{c|}{ Method B } \\
\hline $\begin{array}{c}\text { Time } \\
(\mathrm{min})\end{array}$ & $\begin{array}{c}\text { Volume of } \\
\text { Gas (mL) }\end{array}$ & $\begin{array}{c}\text { Mass of } \\
\text { Gas }(\mathrm{g})\end{array}$ & $\begin{array}{c}\text { Yield } \\
(\%)\end{array}$ & $\begin{array}{c}\text { Time } \\
(\mathrm{min})\end{array}$ & $\begin{array}{c}\text { Volume of } \\
\text { Gas }(\mathrm{mL})\end{array}$ & $\begin{array}{c}\text { Mass of } \\
\text { Gas }(\mathrm{g})\end{array}$ & $\begin{array}{c}\text { Yield } \\
(\%)\end{array}$ \\
\hline 1 & 0 & 0 & 0 & 1 & 0 & 0 & 0 \\
\hline 5 & 0.5 & - & - & 5 & 0.5 & - & - \\
\hline 10 & 0.5 & - & - & 10 & 0.5 & - & - \\
\hline 20 & 0.5 & - & - & 20 & 0.5 & - & - \\
\hline
\end{tabular}

Comments: The substance is known to disproportionate in acidic water to give $\mathrm{Cl}_{2}(\mathrm{~g})$, along with $\mathrm{HClO}_{4}(\mathrm{aq}), \mathrm{O}_{2}(\mathrm{~g})$, and other products. No gases formed when it was mixed with water under these conditions. The dissolution in water was exothermic enough to cause a slight increase (less than $0.5 \mathrm{~mL}$ ) in the volume of the gas-evolution apparatus.

\section{UN 1752 - Chloroacetyl Chloride, $\mathrm{ClCH}_{2} \mathrm{COCl}$}

Chloroacetyl chloride is a liquid with a relative molar mass of 112.94 and a roomtemperature density of $1.418 \mathrm{~g} / \mathrm{cm}^{3}$. In method A, $1.00 \mathrm{mmol}$ of water was added to $1.00 \mathrm{mmol}$ of it. In method B, $1.00 \mathrm{mmol}$ of the substance was added to $5.00 \mathrm{mmol}$ of water. Reaction according to the equation

$$
\mathrm{ClCH}_{2} \mathrm{COCl}+\mathrm{H}_{2} \mathrm{O} \longrightarrow \mathrm{HCl}+\mathrm{ClCH}_{2} \mathrm{COOH}
$$

was taken as the source of $\mathrm{HCl}$. Generation of $1.00 \mathrm{mmol}$ of gas corresponded to an increase in volume of $16.0 \mathrm{~mL}$ in the calibrated apparatus. 
1999 Experimental Results for Chloroacetyl Chloride

\begin{tabular}{|c|c|c|c|c|c|c|c|}
\hline \multicolumn{4}{|c|}{ Method A } & \multicolumn{4}{c|}{ Method B } \\
\hline $\begin{array}{c}\text { Time } \\
(\mathrm{min})\end{array}$ & $\begin{array}{c}\text { Volume of } \\
\text { Gas (mL) }\end{array}$ & $\begin{array}{c}\text { Mass of } \\
\mathrm{HCl}(\mathrm{g})\end{array}$ & $\begin{array}{c}\text { Yield } \\
(\%)\end{array}$ & $\begin{array}{c}\text { Time } \\
(\mathrm{min})\end{array}$ & $\begin{array}{c}\text { Volume of } \\
\text { Gas (mL) }\end{array}$ & $\begin{array}{c}\text { Mass of } \\
\mathrm{HCl}(\mathrm{g})\end{array}$ & $\begin{array}{c}\text { Yield } \\
(\%)\end{array}$ \\
\hline 1 & 0.2 & 0.00046 & 1.2 & 1 & 0.2 & 0.00046 & 1.2 \\
\hline 2 & 0.3 & 0.00068 & 1.9 & 2 & 0.5 & 0.00114 & 3.1 \\
\hline 3 & 0.5 & 0.00114 & 3.1 & 3 & 1.0 & 0.00230 & 6.2 \\
\hline 4 & 0.6 & 0.0014 & 3,7 & 4 & 0.5 & 0.00114 & 3.1 \\
\hline 5 & 0.8 & 0.0018 & 5.0 & 5 & 0.2 & 0.00046 & 1.2 \\
\hline 10 & 2.7 & 0.0062 & 17 & 10 & 0 & 0 & 0 \\
\hline 20 & 6.2 & 0.014 & 39 & 20 & 0 & 0 & 0 \\
\hline 30 & 10.2 & 0.023 & 64 & & & & \\
\hline 40 & 12.0 & 0.027 & 75 & & & & \\
\hline 50 & 13.2 & 0.030 & 82 & & & & \\
\hline 60 & 13.9 & 0.032 & 87 & & & & \\
\hline 90 & 15.4 & 0.035 & 96 & & & & \\
\hline 120 & 15.8 & 0.036 & 99 & & & & \\
\hline 150 & 15.9 & 0.036 & 99 & & & & \\
\hline
\end{tabular}

2003 Experimental Results for Chloroacetyl Chloride

\begin{tabular}{|c|c|c|c|c|c|c|c|}
\hline \multicolumn{4}{|c|}{ Method A } & \multicolumn{4}{c|}{ Method B } \\
\hline $\begin{array}{c}\text { Time } \\
(\mathrm{min})\end{array}$ & $\begin{array}{c}\text { Volume of } \\
\text { Gas (mL) }\end{array}$ & $\begin{array}{c}\text { Mass of } \\
\mathrm{HCl}(\mathrm{g})\end{array}$ & $\begin{array}{c}\text { Yield } \\
(\%)\end{array}$ & $\begin{array}{c}\text { Time } \\
(\mathrm{min})\end{array}$ & $\begin{array}{c}\text { Volume of } \\
\text { Gas (mL) }\end{array}$ & $\begin{array}{c}\text { Mass of } \\
\mathrm{HCl}(\mathrm{g})\end{array}$ & $\begin{array}{c}\text { Yield } \\
(\%)\end{array}$ \\
\hline 1 & 0.2 & 0.00046 & 1.3 & 0.5 & 0.3 & 0.00068 & 1.88 \\
\hline 2 & 0.3 & 0.00068 & 1.9 & 1 & 0.5 & 0.00114 & 3.13 \\
\hline 3 & 0.5 & 0.00114 & 3.1 & 1.5 & 0.7 & 0.00160 & 4.38 \\
\hline 4 & 0.6 & 0.00137 & 3.8 & 2 & 0.9 & 0.00205 & 5.63 \\
\hline 5 & 0.8 & 0.00182 & 5.0 & 2.5 & 1.0 & 0.00228 & 6.25 \\
\hline 10 & 2.7 & 0.0062 & 16.9 & 3 & 1.1 & 0.00251 & 6.88 \\
\hline 20 & 6.2 & 0.0141 & 38.8 & 3.5 & 1.0 & 0.00228 & 6.25 \\
\hline 30 & 10.2 & 0.0232 & 63.8 & 4 & 0.5 & 0.00114 & 3.13 \\
\hline 40 & 12.0 & 0.0273 & 75.0 & 5 & 0.2 & 0.00046 & 1.25 \\
\hline 50 & 13.2 & 0.0301 & 82.5 & 6 & 0.1 & 0.00023 & 0.63 \\
\hline 60 & 13.9 & 0.0317 & 86.9 & 7 & 0.00 & 0.0000 & 0.00 \\
\hline 90 & 15.4 & 0.0351 & 96.3 & & & & \\
\hline 120 & 15.8 & 0.0360 & 98.8 & & & & \\
\hline 150 & 15.9 & 0.0362 & 99.4 & & & & \\
\hline
\end{tabular}

Comments: In method A, the reaction started slowly but picked up speed, suggesting autocatalysis (catalysis by one of the products). In method $\mathrm{B}$, the reaction was fast, and dissolution of $\mathrm{HCl}(\mathrm{g})$ in the excess water was fairly fast; maxima on the $\mathrm{HCl}(\mathrm{g})$ yield curve are evident. In method $\mathrm{B}$, all of the $\mathrm{HCl}$ eventually dissolved in the excess water, but in an open spill 
the duration of contact between $\mathrm{HCl}$ and the water would likely be lesser, and emission might be substantial.

\section{UN 1754 - Chlorosulfonic Acid, $\mathrm{ClSO}_{2}(\mathrm{OH})$}

Chlorosulfonic acid is a liquid with relative molar mass of 116.52 and a roomtemperature density of $1.753 \mathrm{~g} / \mathrm{cm}^{3}$. In method A, $1.00 \mathrm{mmol}$ of it was mixed with $1.00 \mathrm{mmol}$ of water. In method B, $1.00 \mathrm{mmol}$ of it was mixed with $5.00 \mathrm{mmol}$ of water. The equation

$$
\mathrm{ClSO}_{2}(\mathrm{OH})+\mathrm{H}_{2} \mathrm{O} \longrightarrow \mathrm{HCl}+\mathrm{H}_{2} \mathrm{SO}_{4}
$$

was taken to represent the evolution of $\mathrm{HCl}(\mathrm{g})$. The theoretical yield of $\mathrm{HCl}(\mathrm{g})$ was $1.00 \mathrm{mmol}$, which corresponded to $16.0 \mathrm{~mL}$ of gas in the calibrated experimental apparatus.

2003 Experimental Data for Chlorosulfonic Acid

\begin{tabular}{|c|c|c|c|c|c|c|c|}
\hline \multicolumn{4}{|c|}{ Method A } & \multicolumn{4}{c|}{ Method B } \\
\hline $\begin{array}{c}\text { Time } \\
(\mathrm{min})\end{array}$ & $\begin{array}{c}\text { Volume of } \\
\text { Gas (mL) }\end{array}$ & $\begin{array}{c}\text { Mass of } \\
\mathrm{HCl}(\mathrm{g})\end{array}$ & $\begin{array}{c}\text { Yield } \\
(\%)\end{array}$ & $\begin{array}{c}\text { Time } \\
(\mathrm{min})\end{array}$ & $\begin{array}{c}\text { Volume of } \\
\text { Gas (mL) }\end{array}$ & $\begin{array}{c}\text { Mass of } \\
\mathrm{HCl}(\mathrm{g})\end{array}$ & $\begin{array}{c}\text { Yield } \\
(\%)\end{array}$ \\
\hline 0.167 & 11.1 & 0.025 & 69.4 & 0.167 & 15.1 & 0.034 & 94.4 \\
\hline 0.33 & 13.9 & 0.032 & 86.9 & 0.5 & 14.5 & 0.033 & 90.6 \\
\hline 0.5 & 15.4 & 0.035 & 96.3 & 1 & 14.1 & 0.032 & 88.1 \\
\hline 1 & 15.5 & 0.035 & 96.9 & 2 & 13.5 & 0.031 & 84.4 \\
\hline 2 & 15.5 & 0.035 & 96.9 & 3 & 13.0 & 0.030 & 81.3 \\
\hline 5 & 15.6 & 0.036 & 97.5 & 5 & 12.5 & 0.028 & 78.1 \\
\hline & & & & 7 & 12.2 & 0.028 & 76.3 \\
\hline & & & & 10 & 12.0 & 0.027 & 75.0 \\
\hline & & & & 15 & 11.8 & 0.027 & 73.8 \\
\hline & & & & 20 & 11.7 & 0.027 & 73.1 \\
\hline
\end{tabular}

Comments: In method $A$, the reaction was rapid and yielded essentially the theoretical amount of $\mathrm{HCl}(\mathrm{g})$. In method $\mathrm{B}$, hydrolysis was similarly rapid and was followed by a slower dissolution of $\mathrm{HCl}(\mathrm{g})$ in the excess water (as shown by the maximum on the yield curve). The acidic by-product $\mathrm{H}_{2} \mathrm{SO}_{4}$ that was co-dissolved in the excess water certainly lowered the solubility of the $\mathrm{HCl}$ and so raised the yield of $\mathrm{HCl}(\mathrm{g})$. In a real spill, the by-product $\mathrm{H}_{2} \mathrm{SO}_{4}$ would be diluted and the amount of $\mathrm{HCl}(\mathrm{g})$ would be less.

\section{UN 1758 - Chromium Oxychloride, $\mathrm{CrO}_{2} \mathrm{Cl}_{2}$}

Chromium oxychloride, also called chromyl chloride, is a dark-red liquid with a relative molar mass of 154.90 and a room-temperature density of $1.911 \mathrm{~g} / \mathrm{cm}^{3}$. In method A, $1.00 \mathrm{mmol}$ 
of it was mixed with $1.00 \mathrm{mmol}$ of water. In method B, $1.00 \mathrm{mmol}$ of it was mixed with $3.00 \mathrm{mmol}$ (rather than $5.00 \mathrm{mmol}$ ) of water. Reaction according to the equation

$$
\mathrm{CrO}_{2} \mathrm{Cl}_{2}+\mathrm{H}_{2} \mathrm{O} \longrightarrow 2 \mathrm{HCl}+\mathrm{CrO}_{3}
$$

was taken as the source of $\mathrm{HCl}(\mathrm{g})$. The theoretical yield of $\mathrm{HCl}(\mathrm{g})$ was $2.00 \mathrm{mmol}$, which corresponded to $32.0 \mathrm{~mL}$ of gas in the calibrated experimental apparatus.

\section{Experimental Data for Chromium Oxychloride}

\begin{tabular}{|c|c|c|c|c|c|c|c|}
\hline \multicolumn{4}{|c|}{ Method A } & \multicolumn{4}{c|}{ Method B } \\
\hline $\begin{array}{c}\text { Time } \\
(\mathrm{min})\end{array}$ & $\begin{array}{c}\text { Volume of } \\
\text { Gas (mL) }\end{array}$ & $\begin{array}{c}\text { Mass of } \\
\mathrm{HCl}(\mathrm{g})\end{array}$ & $\begin{array}{c}\text { Yield } \\
(\%)\end{array}$ & $\begin{array}{c}\text { Time } \\
(\mathrm{min})\end{array}$ & $\begin{array}{c}\text { Volume of } \\
\text { Gas (mL) }\end{array}$ & $\begin{array}{c}\text { Mass of } \\
\mathrm{HCl}(\mathrm{g})\end{array}$ & $\begin{array}{c}\text { Yield } \\
(\%)\end{array}$ \\
\hline 2 & 0.2 & 0.00046 & 0.6 & 2 & 0.8 & 0.0014 & 1.9 \\
\hline 5 & 0.3 & 0.00068 & 0.9 & 5 & 1.4 & 0.0018 & 2.5 \\
\hline 10 & 0.3 & 0.00068 & 0.9 & 10 & 1.9 & 0.0032 & 4.4 \\
\hline 20 & 0.3 & 0.00068 & 0.9 & 15 & 2.4 & 0.0043 & 5.9 \\
\hline & & & & 20 & 2.8 & 0.0055 & 7.5 \\
\hline & & & & 30 & 2.9 & 0.0064 & 8.8 \\
\hline & & & & 45 & 2.9 & 0.0066 & 9.1 \\
\hline & & & & 60 & 0 & 0.0066 & 9.1 \\
\hline
\end{tabular}

Comments: In method $\mathrm{A}$, the amount of gaseous $\mathrm{HCl}(\mathrm{g})$ that formed was negligibly small - less than $1 \%$ of the theoretical yield. In method B, the rate of hydrolysis of the substance was again very slow, but the yield of $\mathrm{HCl}$ was substantially higher, despite the possible loss of $\mathrm{HCl}(\mathrm{g})$ by dissolution.

\section{UN 1763 - Cyclohexyltrichlorosilane, $\mathrm{C}_{6} \mathrm{H}_{11} \mathrm{SiCl}_{3}$}

Cyclohexyltrichlorosilane is a liquid with a relative molar mass of 217.60 and a roomtemperature density of $1.232 \mathrm{~g} / \mathrm{cm}^{3}$. In method A, $1.00 \mathrm{mmol}$ of it was mixed with $1.50 \mathrm{mmol}$ of water. In method B, $1.00 \mathrm{mmol}$ of it was mixed with $7.50 \mathrm{mmol}$ of water. Reaction according to the equation

$$
\mathrm{C}_{6} \mathrm{H}_{11} \mathrm{SiCl}_{3}+3 / 2 \mathrm{H}_{2} \mathrm{O} \longrightarrow 3 \mathrm{HCl}+\left[\mathrm{C}_{6} \mathrm{H}_{11} \mathrm{SiO}_{3 / 2}\right]_{\mathrm{n}} \text { polymer }
$$

was taken as the source of $\mathrm{HCl}(\mathrm{g})$. The theoretical yield of $\mathrm{HCl}(\mathrm{g})(3.00 \mathrm{mmol})$ corresponded to $48.0 \mathrm{~mL}$ of gas in the calibrated experimental apparatus. 


\begin{tabular}{|c|c|c|c|c|c|c|c|}
\hline \multicolumn{8}{|c|}{2003 Experimental Results for Cyclohexyltrichlorosilane } \\
\hline \multicolumn{4}{|c|}{ Method A } & \multicolumn{4}{|c|}{ Method B } \\
\hline $\begin{array}{l}\text { Time } \\
(\min )\end{array}$ & $\begin{array}{l}\text { Volume of } \\
\text { Gas (mL) }\end{array}$ & $\begin{array}{l}\text { Mass of } \\
\mathrm{HCl}(\mathrm{g})\end{array}$ & $\begin{array}{c}\text { Yield } \\
(\%)\end{array}$ & $\begin{array}{l}\text { Time } \\
\text { (min) }\end{array}$ & $\begin{array}{l}\text { Volume of } \\
\text { Gas (mL) }\end{array}$ & $\begin{array}{l}\text { Mass of } \\
\mathrm{HCl}(\mathrm{g})\end{array}$ & $\begin{array}{c}\text { Yield } \\
(\%)\end{array}$ \\
\hline 1 & 0.2 & 0.00046 & 0.4 & 1 & 0.2 & 0.00046 & 0.4 \\
\hline 2 & 0.3 & 0.00068 & 0.6 & 2 & 0.4 & 0.00091 & 0.8 \\
\hline 3 & 0.4 & 0.00091 & 0.8 & 3 & 0.6 & 0.00137 & 1.3 \\
\hline 4 & 0.6 & 0.00137 & 1.3 & 4 & 0.8 & 0.00182 & 1.7 \\
\hline 5 & 1.0 & 0.0023 & 2.1 & 5 & 1.5 & 0.0034 & 3.1 \\
\hline 6 & 1.4 & 0.0032 & 2.9 & 6 & 3.6 & 0.0082 & 7.5 \\
\hline 7 & 2.0 & 0.0046 & 4.2 & 7 & 5.7 & 0.0130 & 11.9 \\
\hline 8 & 2.5 & 0.0057 & 5.2 & 8 & 7.2 & 0.0164 & 15.0 \\
\hline 9 & 3.0 & 0.0068 & 6.3 & 9 & 8.4 & 0.0191 & 17.5 \\
\hline 10 & 3.7 & 0.0084 & 7.7 & 10 & 9.3 & 0.0212 & 19.4 \\
\hline 12 & 4.9 & 0.0112 & 10.2 & 12 & 10.7 & 0.0244 & 22.3 \\
\hline 15 & 6.6 & 0.0150 & 13.8 & 15 & 11.6 & 0.0264 & 24.2 \\
\hline 20 & 9.2 & 0.0210 & 19.2 & 20 & 12.6 & 0.0287 & 26.3 \\
\hline 25 & 11.7 & 0.027 & 24.4 & 25 & 13.2 & 0.030 & 27.5 \\
\hline 30 & 13.8 & 0.031 & 28.8 & 30 & 13.5 & 0.031 & 28.1 \\
\hline 40 & 17.8 & 0.041 & 37.1 & 35 & 13.7 & 0.031 & 28.5 \\
\hline 50 & 21.7 & 0.049 & 45.2 & 40 & 13.8 & 0.031 & 28.8 \\
\hline 60 & 25.8 & 0.059 & 53.8 & 45 & 13.8 & 0.031 & 28.8 \\
\hline 70 & 29.8 & 0.068 & 62.1 & & & & \\
\hline 80 & 30.8 & 0.070 & 64.2 & & & & \\
\hline 90 & 30.9 & 0.070 & 64.4 & & & & \\
\hline 120 & 31.0 & 0.071 & 64.6 & & & & \\
\hline
\end{tabular}

Comments: In method $\mathrm{A}$, the reaction slowly formed a white solid polysiloxane that sequestered water. In method B, the solid polysiloxane by-product apparently sequestered a large portion of the monomer, thus preventing it from contacting the water.

\section{UN 1766 (Surrogate) - 4-(Chloromethyl)Phenyltrichlorosilane, $\mathrm{ClCH}_{2} \mathrm{C}_{6} \mathrm{H}_{4} \mathrm{SiCl}_{3}$}

4-(Chloromethyl)phenyltrichlorosilane was studied as a surrogate for dichlorophenyltrichlorosilane (UN 1766). It is a liquid with a relative molar mass of 260.02 and a roomtemperature density of $1.361 \mathrm{~g} / \mathrm{cm}^{3}$. In method A, $1.00 \mathrm{mmol}$ of it was mixed with $1.50 \mathrm{mmol}$ of water. In method B, $1.00 \mathrm{mmol}$ of it was mixed with $7.50 \mathrm{mmol}$ of water. Reaction according to the equation

$$
\mathrm{ClCH}_{2} \mathrm{C}_{6} \mathrm{H}_{4} \mathrm{SiCl}_{3}+3 / 2 \mathrm{H}_{2} \mathrm{O} \longrightarrow 3 \mathrm{HCl}+\left[\mathrm{ClCH}_{2} \mathrm{C}_{6} \mathrm{H}_{4} \mathrm{SiO}_{3} / 2\right] \text { n polymer }
$$

was taken as the source of $\mathrm{HCl}(\mathrm{g})$. The theoretical yield of $\mathrm{HCl}(\mathrm{g})(3.00 \mathrm{mmol})$ corresponded to $48.0 \mathrm{~mL}$ of gas in the calibrated experimental apparatus. 


\begin{tabular}{|c|c|c|c|c|c|c|c|}
\hline \multicolumn{8}{|c|}{2003 Experimental Results for 4-(Chloromethyl)Phenyltrichlorosilane } \\
\hline \multicolumn{4}{|c|}{ Method A } & \multicolumn{4}{|c|}{ Method B } \\
\hline $\begin{array}{l}\text { Time } \\
\text { (min) }\end{array}$ & $\begin{array}{l}\text { Volume of } \\
\text { Gas (mL) }\end{array}$ & $\begin{array}{l}\text { Mass of } \\
\mathrm{HCl}(\mathrm{g})\end{array}$ & $\begin{array}{c}\text { Yield } \\
(\%)\end{array}$ & $\begin{array}{l}\text { Time } \\
\text { (min) }\end{array}$ & $\begin{array}{l}\text { Volume of } \\
\text { Gas (mL) }\end{array}$ & $\begin{array}{l}\text { Mass of } \\
\mathrm{HCl}(\mathrm{g})\end{array}$ & $\begin{array}{c}\text { Yield } \\
(\%)\end{array}$ \\
\hline 1 & 0.6 & 0.0014 & 1.3 & 1 & 24.8 & 0.057 & 51.7 \\
\hline 2 & 1.2 & 0.0027 & 2.5 & 2 & 28.5 & 0.065 & 59.4 \\
\hline 3 & 2.1 & 0.0048 & 4.4 & 3 & 28.4 & 0.065 & 59.2 \\
\hline 4 & 2.9 & 0.0066 & 6.0 & 4 & 27.7 & 0.063 & 57.7 \\
\hline 5 & 3.8 & 0.0087 & 7.9 & 5 & 26.9 & 0.061 & 56.0 \\
\hline 6 & 4.8 & 0.0109 & 10.0 & 6 & 26.3 & 0.060 & 54.8 \\
\hline 8 & 6.7 & 0.0153 & 14.0 & 8 & 25.1 & 0.057 & 52.3 \\
\hline 10 & 8.5 & 0.0194 & 17.7 & 10 & 24.0 & 0.055 & 50.0 \\
\hline 12 & 10.0 & 0.0228 & 20.8 & 12 & 23.5 & 0.054 & 49.0 \\
\hline 15 & 11.9 & 0.0271 & 24.8 & 15 & 22.3 & 0.051 & 46.5 \\
\hline 20 & 14.2 & 0.0324 & 29.6 & 20 & 21.0 & 0.048 & 43.8 \\
\hline 25 & 15.9 & 0.0362 & 33.1 & 25 & 20.0 & 0.046 & 41.7 \\
\hline 30 & 16.9 & 0.0385 & 35.2 & 30 & 19.3 & 0.044 & 40.2 \\
\hline 45 & 17.6 & 0.0401 & 36.7 & 45 & 17.3 & 0.039 & 36.0 \\
\hline 60 & 17.7 & 0.0403 & 36.9 & 60 & 15.8 & 0.036 & 32.9 \\
\hline 75 & 17.8 & 0.0406 & 37.1 & 75 & 14.8 & 0.034 & 30.8 \\
\hline \multirow[t]{2}{*}{90} & 17.8 & 0.0406 & 37.1 & 90 & 14.1 & 0.032 & 29.4 \\
\hline & & & & 120 & 13.7 & 0.031 & 28.5 \\
\hline
\end{tabular}

Comments: In method A, the reaction formed a solid white polysiloxane that sequestered water and so restricted the yield of $\mathrm{HCl}(\mathrm{g})$. In method $\mathrm{B}$, the yield of $\mathrm{HCl}(\mathrm{g})$ that was evolved was less than the theoretical amount because of (1) sequestration of some water by the polysiloxane product and (2) dissolution of some $\mathrm{HCl}(\mathrm{g})$ in the excess water to produce $\mathrm{HCl}(\mathrm{aq})$. A maximum on the yield curve of $\mathrm{HCl}(\mathrm{g})$ was reached in $2 \mathrm{~min}$.

\section{UN 1767 - Diethyldichlorosilane, $\left(\mathrm{C}_{2} \mathrm{H}_{5}\right)_{2} \mathrm{SiCl}_{2}$}

Diethyldichlorosilane is a liquid with relative molar mass of 157.12 and a roomtemperature density of $1.050 \mathrm{~g} / \mathrm{cm}^{3}$. In method A, $1.00 \mathrm{mmol}$ of it was mixed with $1.00 \mathrm{mmol}$ of water. In method B, $1.00 \mathrm{mmol}$ of it was mixed with $5.00 \mathrm{mmol}$ of water. Reaction according to the equation

$$
\left(\mathrm{CH}_{3} \mathrm{CH}_{2}\right)_{2} \mathrm{SiCl}_{2}+\mathrm{H}_{2} \mathrm{O} \longrightarrow 2 \mathrm{HCl}+\left[\left(\mathrm{CH}_{3} \mathrm{CH}_{2}\right)_{2} \mathrm{SiO}\right]_{n} \text { polymer }
$$

was taken as the source of $\mathrm{HCl}(\mathrm{g})$. The theoretical yield of $\mathrm{HCl}(\mathrm{g})$ was $2.00 \mathrm{mmol}$, which corresponded to $32.0 \mathrm{~mL}$ of gas in the calibrated experimental apparatus. 
2003 Experimental Data for Diethyldichlorosilane

\begin{tabular}{|c|c|c|c|c|c|c|c|}
\hline \multicolumn{4}{|c|}{ Method A } & \multicolumn{4}{c|}{ Method B } \\
\hline $\begin{array}{c}\text { Time } \\
(\mathrm{min})\end{array}$ & $\begin{array}{c}\text { Volume of } \\
\text { Gas (mL) }\end{array}$ & $\begin{array}{c}\text { Mass of } \\
\text { HCl (g) }\end{array}$ & $\begin{array}{c}\text { Yield } \\
(\%)\end{array}$ & $\begin{array}{c}\text { Time } \\
(\mathrm{min})\end{array}$ & $\begin{array}{c}\text { Volume of } \\
\text { Gas (mL) }\end{array}$ & $\begin{array}{c}\text { Mass of } \\
\text { HCl (g) }\end{array}$ & $\begin{array}{c}\text { Yield } \\
(\%)\end{array}$ \\
\hline 1 & 0.4 & 0.0009 & 1.3 & 1 & 0.2 & 0.0005 & 0.6 \\
\hline 2 & 0.7 & 0.0016 & 2.2 & 2 & 0.4 & 0.0009 & 1.3 \\
\hline 3 & 0.9 & 0.0021 & 2.8 & 3 & 0.6 & 0.0014 & 1.9 \\
\hline 5 & 1.3 & 0.0030 & 4.1 & 5 & 0.8 & 0.0018 & 2.5 \\
\hline 10 & 2.4 & 0.0055 & 7.5 & 7 & 1.3 & 0.0030 & 4.1 \\
\hline 15 & 3.4 & 0.0077 & 10.6 & 10 & 2.0 & 0.0046 & 6.3 \\
\hline 20 & 4.3 & 0.0098 & 13.4 & 12 & 2.4 & 0.0055 & 7.5 \\
\hline 25 & 5.2 & 0.0118 & 16.3 & 15 & 3.0 & 0.0068 & 9.4 \\
\hline 30 & 5.9 & 0.0134 & 18.4 & 20 & 3.8 & 0.0087 & 11.9 \\
\hline 40 & 7.2 & 0.0164 & 22.5 & 25 & 4.4 & 0.0100 & 13.8 \\
\hline 50 & 8.3 & 0.0189 & 25.9 & 30 & 4.9 & 0.0112 & 15.3 \\
\hline 60 & 9.2 & 0.0210 & 28.8 & 40 & 5.6 & 0.0128 & 17.5 \\
\hline 90 & 10.9 & 0.0248 & 34.1 & 50 & 6.1 & 0.0139 & 19.1 \\
\hline 120 & 12.0 & 0.0273 & 37.5 & 60 & 6.4 & 0.0146 & 20.0 \\
\hline 150 & 12.5 & 0.0285 & 39.1 & 90 & 6.6 & 0.0150 & 20.6 \\
\hline 180 & 12.7 & 0.0289 & 39.7 & & & & \\
\hline
\end{tabular}

Comments: In method $\mathrm{A}$, the reaction formed a very viscous liquid by-product polysiloxane. This by-product sequestered a considerable amount of water so that less than half of the stoichiometric amount of $\mathrm{HCl}(\mathrm{g})$ was evolved. In method $\mathrm{B}$, the polymer again formed and remained liquid throughout in the course of the reaction. The presence of this liquid may have accelerated the dissolution of $\mathrm{HCl}(\mathrm{g})$ in the excess water. No maximum on the $\mathrm{HCl}(\mathrm{g})$ yield curve was observed.

\section{UN 1769 - Dichlorodiphenylsilane, $\left(\mathrm{C}_{6} \mathrm{H}_{5}\right)_{2} \mathrm{SiCl}_{2}$}

Dichlorodiphenylsilane is a liquid with a relative molar mass of 253.20 and a roomtemperature density of $1.204 \mathrm{~g} / \mathrm{cm}^{3}$. In method A, $1.00 \mathrm{mmol}$ of it was mixed with $1.00 \mathrm{mmol}$ of water. In method B, $1.00 \mathrm{mmol}$ of it was mixed with $5.00 \mathrm{mmol}$ of water. Reaction according to the equation

$$
\left(\mathrm{C}_{6} \mathrm{H}_{5}\right)_{2} \mathrm{SiCl}_{2}+\mathrm{H}_{2} \mathrm{O} \longrightarrow 2 \mathrm{HCl}+\left[\left(\mathrm{C}_{6} \mathrm{H}_{5}\right)_{2} \mathrm{SiO}\right]_{n} \text { polymer }
$$

was taken as the source of $\mathrm{HCl}(\mathrm{g})$. The theoretical yield of $\mathrm{HCl}(\mathrm{g})$ was $2.00 \mathrm{mmol}$, which corresponded to $32.0 \mathrm{~mL}$ of gas in the calibrated experimental apparatus. 
2003 Experimental Data for Dichlorodiphenylsilane

\begin{tabular}{|c|c|c|c|c|c|c|c|}
\hline \multicolumn{4}{|c|}{ Method A } & \multicolumn{4}{c|}{ Method B } \\
\hline $\begin{array}{c}\text { Time } \\
(\mathrm{min})\end{array}$ & $\begin{array}{c}\text { Volume of } \\
\text { Gas (mL) }\end{array}$ & $\begin{array}{c}\text { Mass of } \\
\mathrm{HCl}(\mathrm{g})\end{array}$ & Yield (\%) & $\begin{array}{c}\text { Time } \\
(\mathrm{min})\end{array}$ & $\begin{array}{c}\text { Volume of } \\
\text { Gas (mL) }\end{array}$ & $\begin{array}{c}\text { Mass of } \\
\mathrm{HCl}(\mathrm{g})\end{array}$ & $\begin{array}{c}\text { Yield } \\
(\%)\end{array}$ \\
\hline 1 & 0.0 & 0.0000 & 0.0 & 1 & 0.3 & 0.0007 & 0.9 \\
\hline 3 & 0.1 & 0.0002 & 0.3 & 2 & 0.5 & 0.0011 & 1.6 \\
\hline 5 & 0.6 & 0.0014 & 1.9 & 3 & 1.3 & 0.0030 & 4.1 \\
\hline 8 & 2.3 & 0.0052 & 7.2 & 4 & 1.7 & 0.0039 & 5.3 \\
\hline 10 & 3.3 & 0.0075 & 10.3 & 5 & 2.0 & 0.0046 & 6.3 \\
\hline 12 & 4.4 & 0.0100 & 13.8 & 6 & 2.2 & 0.0050 & 6.9 \\
\hline 15 & 5.8 & 0.0132 & 18.1 & 8 & 2.5 & 0.0057 & 7.8 \\
\hline 20 & 7.5 & 0.0171 & 23.4 & 10 & 2.9 & 0.0066 & 9.1 \\
\hline 30 & 9.1 & 0.0207 & 28.4 & 12 & 3.1 & 0.0071 & 9.7 \\
\hline 40 & 10.0 & 0.0228 & 31.3 & 15 & 3.5 & 0.0080 & 10.9 \\
\hline 50 & 10.5 & 0.0239 & 33.4 & 30 & 3.9 & 0.0089 & 12.2 \\
\hline 90 & 10.8 & 0.0246 & 33.8 & 40 & 3.9 & 0.0089 & 12.2 \\
\hline
\end{tabular}

Comments: In method $\mathrm{A}$, the reaction slowly formed a solid polysiloxane in its early stages. This material apparently sequestered water, restricting the yield of $\mathrm{HCl}(\mathrm{g})$. In method $\mathrm{B}$, the solid polymer trapped a substantial portion of the reactant. No dissolution of $\mathrm{HCl}(\mathrm{g})$ in the excess water was observed towards the end of the experiment [no maximum on the $\mathrm{HCl}(\mathrm{g})$ yield curve].

\section{UN 1771 - Dodecyltrichlorosilane, $\mathrm{CH}_{3}\left(\mathrm{CH}_{2}\right)_{11} \mathrm{SiCl}_{3}$}

Dodecyltrichlorosilane is a liquid with a relative molar mass of 303.78 and a roomtemperature density of $1.020 \mathrm{~g} / \mathrm{cm}^{3}$. In method A, $1.00 \mathrm{mmol}$ of it was mixed with $1.50 \mathrm{mmol}$ of water. In method B, $1.00 \mathrm{mmol}$ of it was mixed with $7.50 \mathrm{mmol}$ of water. Reaction according to the equation

$$
\mathrm{CH}_{3}\left(\mathrm{CH}_{2}\right)_{11} \mathrm{SiCl}_{3}+3 / 2 \mathrm{H}_{2} \mathrm{O} \longrightarrow 3 \mathrm{HCl}+\left[\mathrm{CH}_{3}\left(\mathrm{CH}_{2}\right)_{11} \mathrm{SiO}_{3 / 2}\right]_{\mathrm{n}} \text { polymer }
$$

was taken as the source of $\mathrm{HCl}(\mathrm{g})$. The theoretical yield of $\mathrm{HCl}(\mathrm{g})(3.00 \mathrm{mmol})$ corresponded to $48.0 \mathrm{~mL}$ of gas in the calibrated experimental apparatus. 


\begin{tabular}{|c|c|c|c|c|c|c|c|}
\hline \multicolumn{8}{|c|}{2003 Experimental Results for Dodecyltrichlorosilane } \\
\hline \multicolumn{4}{|c|}{ Method A } & \multicolumn{4}{|c|}{ Method B } \\
\hline $\begin{array}{l}\text { Time } \\
\text { (min) }\end{array}$ & $\begin{array}{l}\text { Volume of } \\
\text { Gas (mL) }\end{array}$ & $\begin{array}{l}\text { Mass of } \\
\mathrm{HCl}(\mathrm{g})\end{array}$ & $\begin{array}{c}\text { Yield } \\
(\%)\end{array}$ & $\begin{array}{l}\text { Time } \\
\text { (min) }\end{array}$ & $\begin{array}{l}\text { Volume of } \\
\text { Gas (mL) }\end{array}$ & $\begin{array}{l}\text { Mass of } \\
\mathrm{HCl}(\mathrm{g})\end{array}$ & $\begin{array}{c}\text { Yield } \\
(\%)\end{array}$ \\
\hline 1 & 0.3 & 0.0007 & 0.62 & 1 & 1.1 & 0.00525 & 2.3 \\
\hline 2 & 0.5 & 0.0011 & 1.0 & 2 & 2.3 & 0.0052 & 4.8 \\
\hline 3 & 3.5 & 0.0080 & 7.3 & 3 & 4.0 & 0.0091 & 8.3 \\
\hline 4 & 8.8 & 0.0201 & 18.3 & 4 & 4.7 & 0.0107 & 9.8 \\
\hline 5 & 11.8 & 0.0269 & 24.6 & 5 & 5.2 & 0.0119 & 10.8 \\
\hline 6 & 13.3 & 0.0303 & 27.7 & 6 & 5.8 & 0.0132 & 12.1 \\
\hline 8 & 16.3 & 0.0372 & 34.0 & 8 & 7.2 & 0.0164 & 15.0 \\
\hline 10 & 17.7 & 0.0403 & 36.9 & 10 & 8.1 & 0.0185 & 16.9 \\
\hline 12 & 19.1 & 0.0435 & 39.8 & 12 & 9.1 & 0.0207 & 19.0 \\
\hline 15 & 20.5 & 0.0467 & 42.7 & 15 & 10.4 & 0.0237 & 21.7 \\
\hline 20 & 22.4 & 0.0511 & 46.7 & 20 & 12.3 & 0.0280 & 25.6 \\
\hline 25 & 23.6 & 0.538 & 49.2 & 25 & 13.5 & 0.0308 & 28.1 \\
\hline 30 & 24.4 & 0.0556 & 50.8 & 30 & 14.4 & 0.0328 & 30.0 \\
\hline 45 & 25.8 & 0.0588 & 53.8 & 45 & 15.7 & 0.0358 & 32.7 \\
\hline 60 & 26.3 & 0.0599 & 54.8 & 60 & 16.0 & 0.0365 & 33.3 \\
\hline 90 & 26.6 & 0.0606 & 55.4 & 90 & 16.1 & 0.0367 & 33.5 \\
\hline
\end{tabular}

Comments: In method, A the reaction slowly formed a white solid polysiloxane that sequestered some water and so restricted the yield of $\mathrm{HCl}(\mathrm{g})$. In method $\mathrm{B}$, the lesser yield of $\mathrm{HCl}(\mathrm{g})$ (as compared to method A) was caused by dissolution of $\mathrm{HCl}(\mathrm{g})$ in the excess water.

\section{UN 1780 - Fumaryl Chloride, $\mathrm{ClOCCH}=\mathrm{CHCOCI}$}

Fumaryl chloride is a liquid with a relative molar mass of 152.96 and a room-temperature density of $1.41 \mathrm{~g} / \mathrm{cm}^{3}$. In method A, $2.00 \mathrm{mmol}$ of water was added to $1.00 \mathrm{mmol}$ of it. In method B, $1.00 \mathrm{mmol}$ of it was added to $10.0 \mathrm{mmol}$ of water. Reaction according to the equation

\section{$\mathrm{ClOCCH}=\mathrm{CHCOCl}+2 \mathrm{H}_{2} \mathrm{O} \longrightarrow 2 \mathrm{HCl}+\mathrm{HOOCCH}=\mathrm{CHCOOH}$}

was taken as the source of $\mathrm{HCl}$. Generation of $2.00 \mathrm{mmol}$ of gas corresponded to an increase in volume of $32.0 \mathrm{~mL}$ in the calibrated apparatus. 
1999 Experimental Results for Fumaryl Chloride

\begin{tabular}{|c|c|c|c|c|c|c|c|}
\hline \multicolumn{4}{|c|}{ Method A } & \multicolumn{4}{c|}{ Method B } \\
\hline $\begin{array}{c}\text { Time } \\
(\mathrm{min})\end{array}$ & $\begin{array}{c}\text { Volume of } \\
\text { Gas (mL) }\end{array}$ & $\begin{array}{c}\text { Mass of } \\
\mathrm{HCl}(\mathrm{g})\end{array}$ & $\begin{array}{c}\text { Yield } \\
(\%)\end{array}$ & $\begin{array}{c}\text { Time } \\
(\mathrm{min})\end{array}$ & $\begin{array}{c}\text { Volume of } \\
\text { Gas }(\mathrm{mL})\end{array}$ & $\begin{array}{c}\text { Mass of } \\
\mathrm{HCl}(\mathrm{g})\end{array}$ & $\begin{array}{c}\text { Yield } \\
(\%)\end{array}$ \\
\hline 1 & 0 & - & - & 1 & 0.3 & - & - \\
\hline 5 & 0.3 & - & - & 5 & 0.3 & - & - \\
\hline 10 & 0.3 & - & - & 10 & 0 & - & - \\
\hline 20 & 0.3 & - & - & 20 & 0 & - & - \\
\hline
\end{tabular}

Comments: Essentially no gas formation was observed.

\section{UN 1784 - Hexyltrichlorosilane, $\mathrm{C}_{6} \mathrm{H}_{13} \mathrm{SiCl}_{3}$}

Hexyltrichlorosilane is a liquid with a relative molar mass of 219.62 and a roomtemperature density of $1.107 \mathrm{~g} / \mathrm{cm}^{3}$. In method A, $1.00 \mathrm{mmol}$ of it was mixed with $1.50 \mathrm{mmol}$ of water. In method B, $1.00 \mathrm{mmol}$ of it was mixed with $7.50 \mathrm{mmol}$ of water. Reaction according to the equation

$$
\mathrm{C}_{6} \mathrm{H}_{13} \mathrm{SiCl}_{3}+3 / 2 \mathrm{H}_{2} \mathrm{O} \longrightarrow 3 \mathrm{HCl}+\left[\mathrm{C}_{6} \mathrm{H}_{13} \mathrm{SiO}_{3 / 2}\right]_{\mathrm{n}} \text { polymer }
$$

was taken as the source of $\mathrm{HCl}(\mathrm{g})$. The theoretical yield of $\mathrm{HCl}(\mathrm{g})(3.00 \mathrm{mmol})$ corresponded to $48.0 \mathrm{~mL}$ of gas in the calibrated experimental apparatus. 
2003 Experimental Data for Hexyltrichlorosilane

\begin{tabular}{|c|c|c|c|c|c|c|c|}
\hline \multicolumn{4}{|c|}{ Method A } & \multicolumn{4}{c|}{ Method B } \\
\hline $\begin{array}{c}\text { Time } \\
(\mathrm{min})\end{array}$ & $\begin{array}{c}\text { Volume of } \\
\text { Gas (mL) }\end{array}$ & $\begin{array}{c}\text { Mass of } \\
\mathrm{HCl}(\mathrm{g})\end{array}$ & $\begin{array}{c}\text { Yield } \\
(\%)\end{array}$ & $\begin{array}{c}\text { Time } \\
(\mathrm{min})\end{array}$ & $\begin{array}{c}\text { Volume of } \\
\text { Gas (mL) }\end{array}$ & $\begin{array}{c}\text { Mass of } \\
\mathrm{HCl}(\mathrm{g})\end{array}$ & $\begin{array}{c}\text { Yield } \\
(\%)\end{array}$ \\
\hline 1 & 0.5 & 0.0011 & 1.0 & 1 & 0.6 & 0.0014 & 1.3 \\
\hline 2 & 1.0 & 0.0023 & 2.1 & 2 & 1.0 & 0.0023 & 2.1 \\
\hline 3 & 1.4 & 0.0032 & 2.9 & 3 & 1.3 & 0.0030 & 2.7 \\
\hline 4 & 1.8 & 0.0041 & 3.8 & 4 & 1.9 & 0.0043 & 4.0 \\
\hline 5 & 2.1 & 0.0048 & 4.4 & 5 & 2.7 & 0.0062 & 5.6 \\
\hline 6 & 2.4 & 0.0055 & 5.0 & 6 & 4.1 & 0.0093 & 8.5 \\
\hline 8 & 3.0 & 0.0068 & 6.3 & 7 & 5.7 & 0.0130 & 11.9 \\
\hline 10 & 3.6 & 0.0082 & 7.5 & 8 & 7.8 & 0.0178 & 16.3 \\
\hline 12 & 4.2 & 0.0096 & 8.8 & 9 & 10.0 & 0.0228 & 20.8 \\
\hline 15 & 5.2 & 0.0119 & 10.8 & 10 & 13.0 & 0.0296 & 27.1 \\
\hline 20 & 6.9 & 0.0157 & 14.4 & 11 & 15.0 & 0.0342 & 31.3 \\
\hline 30 & 10.2 & 0.0232 & 21.3 & 12 & 16.3 & 0.0372 & 34.0 \\
\hline 40 & 14.4 & 0.033 & 30.0 & 13 & 17.5 & 0.0399 & 36.5 \\
\hline 50 & 18.3 & 0.042 & 38.1 & 15 & 19.1 & 0.0435 & 39.8 \\
\hline 60 & 21.5 & 0.049 & 44.8 & 17 & 19.8 & 0.0451 & 41.3 \\
\hline 70 & 24.8 & 0.057 & 51.7 & 20 & 20.0 & 0.0456 & 41.7 \\
\hline 80 & 26.6 & 0.061 & 55.4 & 30 & 20.0 & 0.0456 & 41.7 \\
\hline 90 & 27.3 & 0.062 & 56.9 & & & & \\
\hline 100 & 27.5 & 0.063 & 57.3 & & & & \\
\hline 120 & 27.6 & 0.063 & 57.5 & & & & \\
\hline 150 & 27.7 & 0.063 & 57.7 & & & & \\
\hline
\end{tabular}

Comments: In method A, The reaction slowly formed a solid polysiloxane that sequestered some water. In method $\mathrm{B}$, the reaction also slowly formed a solid polysiloxane that sequestered some reactant. No maximum was observed on the $\mathrm{HCl}(\mathrm{g})$ yield curve.

\section{UN 1799 - Nonyltrichlorosilane, $\mathrm{C}_{9} \mathrm{H}_{19} \mathrm{SiCl}_{3}$}

Nonyltrichlorosilane is a liquid with a relative molar mass of 261.70 and a roomtemperature density of $1.050 \mathrm{~g} / \mathrm{cm}^{3}$. In method A, $1.00 \mathrm{mmol}$ of it was mixed with $1.50 \mathrm{mmol}$ of water. In method B, $1.00 \mathrm{mmol}$ of it was mixed with $7.50 \mathrm{mmol}$ of water. Reaction according to the equation

$$
\mathrm{C}_{9} \mathrm{H}_{19} \mathrm{SiCl}_{3}+3 / 2 \mathrm{H}_{2} \mathrm{O} \longrightarrow 3 \mathrm{HCl}+\left[\mathrm{C}_{9} \mathrm{H}_{19} \mathrm{SiO}_{3 / 2}\right]_{\mathrm{n}} \text { polymer }
$$

was taken as the source of $\mathrm{HCl}(\mathrm{g})$. The theoretical yield of $\mathrm{HCl}(\mathrm{g})(3.00 \mathrm{mmol})$ corresponded to $48.0 \mathrm{~mL}$ of gas in the calibrated experimental apparatus. 
2003 Experimental Data for Nonyltrichlorosilane

\begin{tabular}{|c|c|c|c|c|c|c|c|}
\hline \multicolumn{4}{|c|}{ Method A } & \multicolumn{4}{c|}{ Method B } \\
\hline $\begin{array}{c}\text { Time } \\
(\mathrm{min})\end{array}$ & $\begin{array}{c}\text { Volume of } \\
\text { Gas (mL) }\end{array}$ & $\begin{array}{c}\text { Mass of } \\
\mathrm{HCl}(\mathrm{g})\end{array}$ & $\begin{array}{c}\text { Yield } \\
(\%)\end{array}$ & $\begin{array}{c}\text { Time } \\
(\mathrm{min})\end{array}$ & $\begin{array}{c}\text { Volume of } \\
\text { Gas (mL) }\end{array}$ & $\begin{array}{c}\text { Mass of } \\
\mathrm{HCl}(\mathrm{g})\end{array}$ & $\begin{array}{c}\text { Yield } \\
(\%)\end{array}$ \\
\hline 1 & 0.9 & 0.0021 & 1.9 & 1 & 1.4 & 0.0032 & 2.9 \\
\hline 2 & 2.0 & 0.0046 & 4.2 & 2 & 2.5 & 0.0057 & 5.2 \\
\hline 3 & 2.8 & 0.0064 & 5.8 & 3 & 3.8 & 0.0087 & 7.9 \\
\hline 4 & 3.4 & 0.0077 & 7.1 & 4 & 4.9 & 0.0112 & 10.2 \\
\hline 5 & 3.8 & 0.0087 & 7.9 & 5 & 5.6 & 0.0128 & 11.7 \\
\hline 7 & 4.6 & 0.0105 & 9.6 & 7 & 6.8 & 0.0155 & 14.2 \\
\hline 10 & 5.5 & 0.0125 & 11.5 & 10 & 8.4 & 0.0191 & 17.5 \\
\hline 15 & 6.8 & 0.0155 & 14.2 & 15 & 10.8 & 0.0246 & 22.5 \\
\hline 20 & 7.6 & 0.0173 & 15.8 & 20 & 13.5 & 0.031 & 28.1 \\
\hline 25 & 8.3 & 0.0189 & 17.3 & 25 & 16.2 & 0.037 & 33.8 \\
\hline 30 & 9.0 & 0.0205 & 18.8 & 30 & 19.3 & 0.044 & 40.2 \\
\hline 40 & 10.1 & 0.0230 & 21.0 & 35 & 22.7 & 0.052 & 47.3 \\
\hline 50 & 11.0 & 0.0251 & 22.9 & 40 & 24.8 & 0.057 & 51.7 \\
\hline 60 & 11.6 & 0.0264 & 24.2 & 45 & 25.7 & 0.059 & 53.5 \\
\hline 75 & 12.3 & 0.0280 & 25.6 & 50 & 26.2 & 0.060 & 54.6 \\
\hline 90 & 13.0 & 0.0296 & 27.1 & 60 & 26.4 & 0.060 & 55.0 \\
\hline 120 & 13.9 & 0.0317 & 29.0 & 90 & 26.5 & 0.060 & 55.2 \\
\hline 150 & 14.2 & 0.0324 & 29.6 & & & & \\
\hline 180 & 14.4 & 0.0328 & 30.0 & & & & \\
\hline
\end{tabular}

Comments: In method $\mathrm{A}$, the reaction formed a viscous polymeric siloxane that apparently sequestered water. In method $\mathrm{B}$, dissolution of $\mathrm{HCl}(\mathrm{g})$ in excess water was not observed towards the end of the reaction [no maximum on the $\mathrm{HCl}(\mathrm{g})$ yield curve].

\section{UN 1800 - Octadecyltrichlorosilane, $\mathrm{C}_{18} \mathrm{H}_{37} \mathrm{SiCl}_{3}$}

Octadecyltrichlorosilane is a liquid with a relative molar mass of 387.94 and a roomtemperature density of $0.984 \mathrm{~g} / \mathrm{cm}^{3}$. In method A, $1.00 \mathrm{mmol}$ of it was mixed with $1.50 \mathrm{mmol}$ of water. In method B, $1.00 \mathrm{mmol}$ of it was mixed with $7.50 \mathrm{mmol}$ of water. Reaction according to the equation

$$
\mathrm{C}_{18} \mathrm{H}_{37} \mathrm{SiCl}_{3}+3 / 2 \mathrm{H}_{2} \mathrm{O} \longrightarrow 3 \mathrm{HCl}+\left[\mathrm{C}_{18} \mathrm{H}_{37} \mathrm{SiO}_{3 / 2}\right]_{\text {n }} \text { polymer }
$$

was taken as the source of $\mathrm{HCl}(\mathrm{g})$. The theoretical yield of $\mathrm{HCl}(\mathrm{g})(3.00 \mathrm{mmol})$ corresponded to $48.0 \mathrm{~mL}$ of gas in the calibrated experimental apparatus. 
2003 Experimental Data for Octadecyltrichlorosilane

\begin{tabular}{|c|c|c|c|c|c|c|c|}
\hline \multicolumn{4}{|c|}{ Method A } & \multicolumn{4}{c|}{ Method B } \\
\hline $\begin{array}{c}\text { Time } \\
(\mathrm{min})\end{array}$ & $\begin{array}{c}\text { Volume of } \\
\text { Gas (mL) }\end{array}$ & $\begin{array}{c}\text { Mass of } \\
\mathrm{HCl}(\mathrm{g})\end{array}$ & $\begin{array}{c}\text { Yield } \\
(\%)\end{array}$ & $\begin{array}{c}\text { Time } \\
(\mathrm{min})\end{array}$ & $\begin{array}{c}\text { Volume of } \\
\text { Gas (mL) }\end{array}$ & $\begin{array}{c}\text { Mass of } \\
\mathrm{HCl}(\mathrm{g})\end{array}$ & $\begin{array}{c}\text { Yield } \\
(\%)\end{array}$ \\
\hline 1 & 0.0 & 0.00 & 0.0 & 1 & 0.0 & 0.00 & 0.0 \\
\hline 2 & 0.0 & 0.00 & 0.0 & 2 & 0.0 & 0.00 & 0.0 \\
\hline 4 & 0.0 & 0.00 & 0.0 & 4 & 0.0 & 0.00 & 0.0 \\
\hline 6 & 0.0 & 0.00 & 0.0 & 6 & 0.0 & 0.00 & 0.0 \\
\hline 8 & 0.0 & 0.00 & 0.0 & 8 & 0.5 & 0.0011 & 1.0 \\
\hline 10 & 0.0 & 0.00 & 0.0 & 9 & 3.0 & 0.0068 & 6.3 \\
\hline 12 & 0.3 & 0.0007 & 0.6 & 10 & 6.9 & 0.0157 & 14.4 \\
\hline 14 & 0.9 & 0.0021 & 1.9 & 11 & 11.2 & 0.0255 & 23.3 \\
\hline 16 & 2.3 & 0.0052 & 4.8 & 12 & 14.7 & 0.034 & 30.6 \\
\hline 18 & 3.9 & 0.0089 & 8.1 & 13 & 17.4 & 0.040 & 36.3 \\
\hline 20 & 5.4 & 0.0123 & 11.3 & 14 & 19.3 & 0.044 & 40.2 \\
\hline 22 & 6.6 & 0.0150 & 13.8 & 15 & 20.8 & 0.047 & 43.3 \\
\hline 25 & 8.5 & 0.0194 & 17.7 & 16 & 22.0 & 0.050 & 45.8 \\
\hline 30 & 11.0 & 0.0251 & 22.9 & 17 & 22.9 & 0.052 & 47.7 \\
\hline 35 & 12.8 & 0.0292 & 26.7 & 18 & 23.6 & 0.054 & 49.2 \\
\hline 40 & 14.5 & 0.033 & 30.2 & 20 & 24.0 & 0.055 & 50.0 \\
\hline 45 & 15.5 & 0.035 & 32.3 & 22 & 24.1 & 0.055 & 50.2 \\
\hline 50 & 16.4 & 0.037 & 34.2 & 25 & 24.2 & 0.055 & 50.4 \\
\hline 60 & 18.4 & 0.042 & 38.3 & 30 & 24.2 & 0.055 & 50.4 \\
\hline 75 & 19.8 & 0.045 & 41.3 & & & & \\
\hline 90 & 20.0 & 0.046 & 41.7 & & & & \\
\hline
\end{tabular}

Comments: In method $\mathrm{A}$, the evolution of gas began only after an induction period, suggesting that the reaction was autocatalytic. A solid polysiloxane formed. Evidently, some water was prevented from reaction by being bound by this by-product. In method B, the reaction got under way slowly. No dissolution of $\mathrm{HCl}(\mathrm{g})$ in excess water was observed towards the end of the reaction [no maximum on the $\mathrm{HCl}(\mathrm{g})$ yield curve].

\section{UN 1801 - Octyltrichlorosilane, $\mathrm{C}_{8} \mathrm{H}_{17} \mathrm{SiCl}_{3}$}

Octyltrichlorosilane is a liquid with a relative molar mass of 247.67 and a roomtemperature density of $1.070 \mathrm{~g} / \mathrm{cm}^{3}$. In method A, $1.00 \mathrm{mmol}$ of it was mixed with $1.50 \mathrm{mmol}$ of water. In method B, $1.00 \mathrm{mmol}$ of it was mixed with $7.50 \mathrm{mmol}$ of water. Reaction according to the equation

$$
\mathrm{C}_{8} \mathrm{H}_{17} \mathrm{SiCl}_{3}+3 / 2 \mathrm{H}_{2} \mathrm{O} \longrightarrow 3 \mathrm{HCl}+\left[\mathrm{C}_{8} \mathrm{H}_{17} \mathrm{SiO}_{3 / 2}\right]_{\mathrm{n}} \text { polymer }
$$

was taken as the source of $\mathrm{HCl}(\mathrm{g})$. The theoretical yield of $\mathrm{HCl}(\mathrm{g})$ was $3.00 \mathrm{mmol}$, which corresponded to $48.0 \mathrm{~mL}$ of gas in the calibrated experimental apparatus. 


\section{Experimental Data for Octyltrichlorosilane}

\begin{tabular}{|c|c|c|c|c|c|c|c|}
\hline \multicolumn{3}{|c|}{ Method A } & \multicolumn{4}{c|}{ Method B } \\
\hline $\begin{array}{c}\text { Time } \\
(\mathrm{min})\end{array}$ & $\begin{array}{c}\text { Volume of } \\
\text { Gas (mL) }\end{array}$ & $\begin{array}{c}\text { Mass of } \\
\mathrm{HCl}(\mathrm{g})\end{array}$ & $\begin{array}{c}\text { Yield } \\
(\%)\end{array}$ & $\begin{array}{c}\text { Time } \\
(\mathrm{min})\end{array}$ & $\begin{array}{c}\text { Volume of } \\
\text { Gas (mL) }\end{array}$ & $\begin{array}{c}\text { Mass of } \\
\mathrm{HCl}(\mathrm{g})\end{array}$ & $\begin{array}{c}\text { Yield } \\
(\%)\end{array}$ \\
\hline 1 & 2.8 & 0.0064 & 5.8 & 1 & 2.6 & 0.0059 & 5.4 \\
\hline 2 & 4.4 & 0.0100 & 9.2 & 2 & 3.8 & 0.0087 & 7.9 \\
\hline 3 & 5.3 & 0.0121 & 11.0 & 3 & 4.5 & 0.0103 & 9.4 \\
\hline 4 & 6.5 & 0.0148 & 13.5 & 4 & 5.0 & 0.0114 & 10.4 \\
\hline 5 & 7.2 & 0.0164 & 15.0 & 5 & 5.7 & 0.0130 & 11.9 \\
\hline 7 & 8.7 & 0.0198 & 18.1 & 7 & 7.0 & 0.0160 & 14.6 \\
\hline 10 & 10.6 & 0.0242 & 22.1 & 10 & 9.5 & 0.0217 & 19.8 \\
\hline 15 & 13.2 & 0.0301 & 27.5 & 15 & 12.1 & 0.0276 & 25.2 \\
\hline 20 & 15.5 & 0.035 & 32.3 & 20 & 13.4 & 0.031 & 27.9 \\
\hline 25 & 17.5 & 0.040 & 36.5 & 30 & 14.2 & 0.032 & 29.6 \\
\hline 30 & 19.3 & 0.044 & 40.2 & 40 & 14.3 & 0.033 & 29.8 \\
\hline 45 & 22.6 & 0.052 & 47.1 & 50 & 14.3 & 0.033 & 29.8 \\
\hline 60 & 24.9 & 0.057 & 51.9 & & & & \\
\hline 75 & 26.2 & 0.060 & 54.6 & & & & \\
\hline 90 & 29.1 & 0.066 & 60.6 & & & & \\
\hline 120 & 31.1 & 0.071 & 64.8 & & & & \\
\hline 150 & 31.1 & 0.071 & 64.8 & & & & \\
\hline
\end{tabular}

Comments: In method A, the substance slowly reacted to form a viscous polymeric siloxane that sequestered some water and about two-thirds of the theoretical amount of $\mathrm{HCl}(\mathrm{g})$. In method B, a solid polymer formed in the early stage of the reaction. This material trapped a portion of the monomer, preventing it from reacting with water. No absorption of $\mathrm{HCl}(\mathrm{g})$ in excess water was observed towards the end of the reaction [i.e., there was no maximum on the $\mathrm{HCl}(\mathrm{g})$ yield curve].

\section{UN 1804 - Phenyltrichlorosilane, $\mathrm{C}_{6} \mathrm{H}_{5} \mathrm{SiCl}_{3}$}

Phenyltrichlorosilane is a liquid with a relative molar mass of 211.55 and a roomtemperature density of $1.321 \mathrm{~g} / \mathrm{cm}^{3}$. In method A, $1.00 \mathrm{mmol}$ of it was mixed with $1.50 \mathrm{mmol}$ of water. In method B, $1.00 \mathrm{mmol}$ of it was mixed with $7.50 \mathrm{mmol}$ of water. Reaction according to the equation

$$
\mathrm{C}_{6} \mathrm{H}_{5} \mathrm{SiCl}_{3}+3 / 2 \mathrm{H}_{2} \mathrm{O} \longrightarrow 3 \mathrm{HCl}+\left[\mathrm{C}_{6} \mathrm{H}_{5} \mathrm{SiO}_{3 / 2}\right]_{\mathrm{n}} \text { polymer }
$$

was taken as the source of $\mathrm{HCl}(\mathrm{g})$. The theoretical yield of $\mathrm{HCl}(\mathrm{g})(3.00 \mathrm{mmol})$ corresponded to $48.0 \mathrm{~mL}$ of gas in the calibrated experimental apparatus. 
2003 Experimental Results for Phenyltrichlorosilane

\begin{tabular}{|c|c|c|c|c|c|c|c|}
\hline \multicolumn{4}{|c|}{ Method A } & \multicolumn{4}{c|}{ Method B } \\
\hline $\begin{array}{c}\text { Time } \\
(\mathrm{min})\end{array}$ & $\begin{array}{c}\text { Volume of } \\
\text { Gas (mL) }\end{array}$ & $\begin{array}{c}\text { Mass of } \\
\mathrm{HCl}(\mathrm{g})\end{array}$ & $\begin{array}{c}\text { Yield } \\
(\%)\end{array}$ & $\begin{array}{c}\text { Time } \\
(\mathrm{min})\end{array}$ & $\begin{array}{c}\text { Volume of } \\
\text { Gas (mL) }\end{array}$ & $\begin{array}{c}\text { Mass of } \\
\text { HCl (g) }\end{array}$ & $\begin{array}{c}\text { Yield } \\
(\%)\end{array}$ \\
\hline 1 & 0.5 & 0.0011 & 1.0 & 1 & 7.1 & 0.016 & 14.8 \\
\hline 2 & 0.9 & 0.0021 & 1.9 & 2 & 21.0 & 0.054 & 43.8 \\
\hline 3 & 1.5 & 0.0034 & 3.1 & 3 & 23.8 & 0.109 & 49.6 \\
\hline 4 & 2.1 & 0.0048 & 4.4 & 4 & 24.5 & 0.056 & 51.0 \\
\hline 5 & 2.9 & 0.0066 & 6.0 & 5 & 24.5 & 0.056 & 51.0 \\
\hline 6 & 3.6 & 0.0082 & 7.5 & 6 & 24.4 & 0.056 & 50.8 \\
\hline 7 & 4.2 & 0.0096 & 8.8 & 7 & 24.2 & 0.055 & 50.4 \\
\hline 8 & 4.9 & 0.0112 & 10.2 & 8 & 24.0 & 0.055 & 50.0 \\
\hline 9 & 5.5 & 0.0125 & 11.5 & 9 & 23.9 & 0.054 & 49.8 \\
\hline 10 & 6.2 & 0.0141 & 12.9 & 10 & 23.8 & 0.054 & 49.6 \\
\hline 12 & 7.5 & 0.0171 & 15.6 & 12 & 23.2 & 0.053 & 48.3 \\
\hline 15 & 9.3 & 0.0212 & 19.4 & 15 & 22.8 & 0.052 & 47.5 \\
\hline 20 & 12.0 & 0.0274 & 25.0 & 20 & 21.9 & 0.050 & 45.6 \\
\hline 25 & 16.0 & 0.0365 & 33.3 & 30 & 20.5 & 0.048 & 42.7 \\
\hline 30 & 22.4 & 0.0511 & 46.7 & 45 & 18.5 & 0.042 & 38.5 \\
\hline 35 & 39.5 & 0.0900 & 82.3 & 60 & 16.8 & 0.038 & 35.0 \\
\hline 40 & 39.6 & 0.0903 & 82.5 & 90 & 14.1 & 0.032 & 29.4 \\
\hline 50 & 39.6 & 0.0903 & 82.5 & 120 & 12.2 & 0.028 & 25.4 \\
\hline & & & & 180 & 10.5 & 0.024 & 21.9 \\
\hline & & & & 240 & 9.8 & 0.022 & 20.4 \\
\hline
\end{tabular}

Comments: In method A, The reaction formed a voluminous white solid polysiloxane but also produced gaseous $\mathrm{HCl}(\mathrm{g})$ in good yield $(82 \%)$. In the final stages, the rate of reaction suddenly increased, nearly doubling the amount of $\mathrm{HCl}(\mathrm{g})$ within a 5-min interval. In method $\mathrm{B}$, the yield of $\mathrm{HCl}(\mathrm{g})$ was less than the theoretical amount because of sequestration of some reactant by the polysiloxane by-product and dissolution of $\mathrm{HCl}(\mathrm{g})$ in the excess water. A maximum on the yield curve of $\mathrm{HCl}(\mathrm{g})$ was reached in $5 \mathrm{~min}$.

\section{UN 1806 - Phosphorus Pentachloride, $\mathrm{PCl}_{5}$}

Phosphorus pentachloride is a solid with a relative molar mass of 208.24. In method A, $4.00 \mathrm{mmol}$ of water was added to $1.00 \mathrm{mmol}$ of it. In method $\mathrm{B}, 1.00 \mathrm{mmol}$ of it was added to $20.0 \mathrm{mmol}$ of water. The reaction

$$
\mathrm{PCl}_{5}+4 \mathrm{H}_{2} \mathrm{O} \longrightarrow 5 \mathrm{HCl}+\mathrm{H}_{3} \mathrm{PO}_{4}
$$

was taken as the source of evolved gas. Generation of $5.00 \mathrm{mmol}$ of gas corresponded to an increase in volume of $80.0 \mathrm{~mL}$ in the calibrated apparatus. 
2003 Experimental Data for Phosphorus Pentachloride

\begin{tabular}{|c|c|c|c|c|c|c|c|}
\hline \multicolumn{4}{|c|}{ Method A } & \multicolumn{4}{c|}{ Method B } \\
\hline $\begin{array}{c}\text { Time } \\
(\mathrm{min})\end{array}$ & $\begin{array}{c}\text { Volume of } \\
\text { Gas }(\mathrm{mL})\end{array}$ & $\begin{array}{c}\text { Mass of } \\
\mathrm{HCl}(\mathrm{g})\end{array}$ & $\begin{array}{c}\text { Yield } \\
(\%)\end{array}$ & $\begin{array}{c}\text { Time } \\
(\mathrm{min})\end{array}$ & $\begin{array}{c}\text { Volume of } \\
\text { Gas }(\mathrm{mL})\end{array}$ & $\begin{array}{c}\text { Mass of } \\
\mathrm{HCl}(\mathrm{g})\end{array}$ & $\begin{array}{c}\text { Yield } \\
(\%)\end{array}$ \\
\hline 0.17 & 30.0 & 0.068 & 37.5 & 0.17 & 18.0 & 0.0410 & 22.5 \\
\hline 0.33 & 35.0 & 0.080 & 43.8 & 0.33 & 11.0 & 0.0251 & 13.8 \\
\hline 1 & 38.2 & 0.087 & 47.8 & 0.5 & 9.6 & 0.0219 & 12.0 \\
\hline 2 & 41.6 & 0.095 & 52.0 & 1 & 6.8 & 0.0155 & 8.5 \\
\hline 3 & 43.0 & 0.098 & 53.8 & 1.5 & 4.8 & 0.0109 & 6.0 \\
\hline 5 & 44.4 & 0.101 & 55.5 & 2 & 3.4 & 0.0077 & 4.3 \\
\hline 7 & 45.6 & 0.104 & 57.0 & 3 & 2.1 & 0.0048 & 2.6 \\
\hline 10 & 45.8 & 0.104 & 57.3 & 5 & 1.0 & 0.0023 & 1.3 \\
\hline 15 & 45.9 & 0.105 & 57.4 & 10 & 0.0 & 0.0000 & 0.0 \\
\hline
\end{tabular}

Comments: In method $\mathrm{A}$, more than $1 \mathrm{mmol}$ of $\mathrm{HCl}(\mathrm{g})$ appeared within seconds. This confirmed that more than one $\mathrm{Cl}$ hydrolyzed per $\mathrm{PCl}_{5}$ molecule. The reaction, however, remained incomplete after $90 \mathrm{~min}$ with respect to the theoretical yield of $5.0 \mathrm{mmol}$ of $\mathrm{HCl}(\mathrm{g})$. In method $\mathrm{B}, \mathrm{HCl}(\mathrm{g})$ also formed very rapidly. Visible signs of reaction vanished within the first minute. This was followed by dissolution of $\mathrm{HCl}(\mathrm{g})$ in the excess water (as shown by the maximum on the yield curve).

\section{UN 1808 - Phosphorus Tribromide, PBr $_{3}$}

Phosphorus tribromide is a liquid with a relative molar mass of 270.68 and a roomtemperature density of $2.85 \mathrm{~g} / \mathrm{cm}^{3}$. In method A, $3.00 \mathrm{mmol}$ of water was added to $1.00 \mathrm{mmol}$ of the substance. In method B, $1.00 \mathrm{mmol}$ of it was added to $15.0 \mathrm{mmol}$ of water. Reaction according to the equation

$$
\mathrm{PBr}_{3}+3 \mathrm{H}_{2} \mathrm{O} \longrightarrow 3 \mathrm{HBr}+\mathrm{P}(\mathrm{OH})_{3}
$$

was taken as the source of $\mathrm{HBr}$. Generation of $3.00 \mathrm{mmol}$ of gas corresponded to an increase in volume of $48.0 \mathrm{~mL}$ in the calibrated apparatus. 
1999 Experimental Results for Phosphorus Tribromide

\begin{tabular}{|c|c|c|c|c|c|c|c|}
\hline \multicolumn{4}{|c|}{ Method A } & \multicolumn{4}{c|}{ Method B } \\
\hline $\begin{array}{c}\text { Time } \\
(\mathrm{min})\end{array}$ & $\begin{array}{c}\text { Volume of } \\
\text { Gas }(\mathrm{mL})\end{array}$ & $\begin{array}{c}\text { Mass of } \\
\mathrm{HBr}(\mathrm{g})\end{array}$ & $\begin{array}{c}\text { Yield } \\
(\%)\end{array}$ & $\begin{array}{c}\text { Time } \\
(\mathrm{min})\end{array}$ & $\begin{array}{c}\text { Volume of } \\
\text { Gas (mL) }\end{array}$ & $\begin{array}{c}\text { Mass of } \\
\mathrm{HBr}(\mathrm{g})\end{array}$ & $\begin{array}{c}\text { Yield } \\
(\%)\end{array}$ \\
\hline 1 & 1.6 & 0.0081 & 3.3 & 1 & 0 & 0 & 0 \\
\hline 2 & 4 & 0.0202 & 8.3 & 2 & 0 & 0 & 0 \\
\hline 3 & 8 & 0.0405 & 17 & 3 & 0 & 0 & 0 \\
\hline 5 & 16 & 0.0809 & 33 & 5 & 0 & 0 & 0 \\
\hline 10 & 32 & 0.162 & 67 & 10 & 0 & 0 & 0 \\
\hline 20 & 43 & 0.217 & 90 & 20 & 0 & 0 & 0 \\
\hline 25 & 45 & 0.228 & 94 & 25 & 0 & 0 & 0 \\
\hline 30 & 46 & 0.233 & 96 & 30 & 0 & 0 & 0 \\
\hline 60 & 47 & 0.238 & 98 & 60 & 0 & 0 & 0 \\
\hline
\end{tabular}

Comments: In method $\mathrm{A}$, the reaction slowly generated $\mathrm{HBr}(\mathrm{g})$ and after an hour had liberated essentially the full theoretical amount. In method B, in the presence of excess water, the solubility of $\mathrm{HBr}$ in water was never exceeded, and the rate of solution of the $\mathrm{HBr}$ was so high that the gas dissolved before it could be released. Therefore, the substance was considered a nonTIHWR chemical, even though in the exothermic, violent conditions of a large spill, some $\mathrm{HBr}$ might well be released as a gas.

\section{UN 1809 - Phosphorus Trichloride, $\mathbf{P C l}_{3}$}

Phosphorus trichloride is a liquid with a relative molar mass of 137.33. In method A, $3.00 \mathrm{mmol}$ of water was added to $1.00 \mathrm{mmol}$ of it. In method B, $1.00 \mathrm{mmol}$ of it was added to $15.0 \mathrm{mmol}$ of water. The reaction

$$
\mathrm{PCl}_{3}+3 \mathrm{H}_{2} \mathrm{O} \longrightarrow 3 \mathrm{HCl}+\mathrm{P}(\mathrm{OH})_{3}
$$

was taken as the source of $\mathrm{HCl}$. Generation of $2.00 \mathrm{mmol}$ of $\mathrm{HCl}(\mathrm{g})$ corresponded to an increase in volume of $48.0 \mathrm{~mL}$ in the calibrated apparatus. 
1999 Experimental Results for Phosphorus Trichloride

\begin{tabular}{|c|c|c|c|c|c|c|c|}
\hline \multicolumn{4}{|c|}{ Method A } & \multicolumn{4}{c|}{ Method B } \\
\hline $\begin{array}{c}\text { Time } \\
(\mathrm{min})\end{array}$ & $\begin{array}{c}\text { Volume of } \\
\text { Gas (mL) }\end{array}$ & $\begin{array}{c}\text { Mass of } \mathrm{HCl} \\
(\mathrm{g})\end{array}$ & $\begin{array}{c}\text { Yield } \\
(\%)\end{array}$ & $\begin{array}{c}\text { Time } \\
(\mathrm{min})\end{array}$ & $\begin{array}{c}\text { Volume of } \\
\text { Gas (mL) }\end{array}$ & $\begin{array}{c}\text { Mass of } \\
\mathrm{HCl}(\mathrm{g})\end{array}$ & $\begin{array}{c}\text { Yield } \\
(\%)\end{array}$ \\
\hline 1 & 26 & 0.059 & 54 & 1 & 16 & 0.036 & 33 \\
\hline 2 & 40 & 0.091 & 83 & 2 & 9 & 0.021 & 19 \\
\hline 3 & 44 & 0.100 & 92 & 3 & 5 & 0.0114 & 10 \\
\hline 5 & 47 & 0.107 & 98 & 5 & 2 & 0.0046 & 4 \\
\hline 10 & 48 & 0.109 & 100 & 10 & 1 & 0.0023 & 2 \\
\hline 20 & 48 & 0.109 & 100 & 20 & 0.5 & 0.0011 & 1 \\
\hline
\end{tabular}

2003 Experimental Results for Phosphorus Trichloride

\begin{tabular}{|c|c|c|c|c|c|c|c|}
\hline \multicolumn{4}{|c|}{ Method A } & \multicolumn{4}{c|}{ Method B } \\
\hline $\begin{array}{c}\text { Time } \\
(\mathrm{min})\end{array}$ & $\begin{array}{c}\text { Volume of } \\
\text { Gas (mL) }\end{array}$ & $\begin{array}{c}\text { Mass of } \\
\text { HCl (g) }\end{array}$ & $\begin{array}{c}\text { Yield } \\
(\%)\end{array}$ & $\begin{array}{c}\text { Time } \\
(\mathrm{min})\end{array}$ & $\begin{array}{c}\text { Volume of } \\
\text { Gas (mL) }\end{array}$ & $\begin{array}{c}\text { Mass of } \\
\mathrm{HCl}(\mathrm{g})\end{array}$ & $\begin{array}{c}\text { Yield } \\
(\%)\end{array}$ \\
\hline 0.5 & 17.5 & 0.0399 & 36.5 & 0.5 & 11.8 & 0.0269 & 24.6 \\
\hline 1 & 25.1 & 0.0572 & 52.3 & 1 & 16.8 & 0.0383 & 35.0 \\
\hline 2 & 39.1 & 0.089 & 81.5 & 2 & 9.4 & 0.0214 & 19.6 \\
\hline 3 & 43.2 & 0.098 & 90.0 & 3 & 5.2 & 0.0118 & 10.8 \\
\hline 4 & 45.6 & 0.104 & 95.0 & 4 & 3.4 & 0.0077 & 7.1 \\
\hline 5 & 46.7 & 0.106 & 97.3 & 5 & 2.2 & 0.0050 & 4.6 \\
\hline 7 & 47.3 & 0.108 & 98.5 & 7 & 1.4 & 0.0032 & 2.9 \\
\hline 10 & 48.0 & 0.109 & 100 & 10 & 1.1 & 0.0025 & 2.3 \\
\hline 20 & 48.0 & 0.109 & 100 & 20 & 0.5 & 0.0011 & 1.0 \\
\hline & & & & 30 & 0.2 & 0.0005 & 0.4 \\
\hline & & & & 60 & 0.1 & 0.0002 & 0.2 \\
\hline
\end{tabular}

Comments: The experiments were repeated in 2003 to obtain more data on the early stages of the reaction. In method $\mathrm{A}$, the theoretical amount of the $\mathrm{HCl}(\mathrm{g})$ was generated in 10 min. In method $\mathrm{B}$, essentially all of the $\mathrm{HCl}(\mathrm{g})$ that formed soon dissolved in the excess water to produce $\mathrm{HCl}(\mathrm{aq})$ (maxima on the yield curves). In the free atmosphere, probably close to onethird of the maximum yield of $\mathrm{HCl}$ would occur, because the gas would not remain in contact with the water for enough time (on the order of $1 \mathrm{~min}$ ) to permit dissolution.

\section{UN 1810 - Phosphorus Oxychloride, POCl$_{3}$}

Phosphorus oxychloride, also called phosphoryl chloride, is a liquid with a relative molar mass of 153.33 and a room-temperature density of $1.675 \mathrm{~g} / \mathrm{cm}^{3}$. In method A, $3.00 \mathrm{mmol}$ of water was added to $1.00 \mathrm{mmol}$ of it. In method B, $1.00 \mathrm{mmol}$ was added to $15.0 \mathrm{mmol}$ of water. Reaction according to the equation 


$$
\mathrm{OPCl}_{3}+3 \mathrm{H}_{2} \mathrm{O} \longrightarrow 3 \mathrm{HCl}+\mathrm{OP}(\mathrm{OH})_{3}
$$

was taken as the source of evolved gas. Generation of $3.00 \mathrm{mmol}$ of gas corresponded to an increase in volume of $48.0 \mathrm{~mL}$ in the calibrated apparatus.

1999 Experimental Results for Phosphorus Oxychloride

\begin{tabular}{|c|c|c|c|c|c|c|c|}
\hline \multicolumn{4}{|c|}{ Method A } & \multicolumn{4}{c|}{ Method B } \\
\hline $\begin{array}{c}\text { Time } \\
(\mathrm{min})\end{array}$ & $\begin{array}{c}\text { Volume of } \\
\text { Gas (mL) }\end{array}$ & $\begin{array}{c}\text { Mass of } \\
\mathrm{HCl}(\mathrm{g})\end{array}$ & $\begin{array}{c}\text { Yield } \\
(\%)\end{array}$ & Time & $\begin{array}{c}\text { Volume } \\
\text { of Gas }\end{array}$ & $\begin{array}{c}\text { Mass of } \\
\mathrm{HCl}(\mathrm{g})\end{array}$ & $\begin{array}{c}\text { Yield } \\
(\%)\end{array}$ \\
\hline 1 & 7 & 0.016 & 15 & 1 & 10 & 0.0228 & 21 \\
\hline 2 & 7.5 & 0.0171 & 16 & 2 & 4.5 & 0.0103 & 9.4 \\
\hline 3 & 8 & 0.0182 & 17 & 3 & 3 & 0.0068 & 6.3 \\
\hline 5 & 8.5 & 0.0194 & 18 & 5 & 2 & 0.0046 & 4.2 \\
\hline 10 & 9 & 0.0205 & 19 & 10 & 1.9 & 0.0043 & 4.0 \\
\hline 20 & 10 & 0.0228 & 21 & 20 & 1.9 & 0.0043 & 4.0 \\
\hline 30 & 11 & 0.0251 & 23 & & & & \\
\hline 60 & 12 & 0.0274 & 25 & & & & \\
\hline 180 & 13 & 0.0297 & 27 & & & & \\
\hline
\end{tabular}

2003 Experimental Results for Phosphorus Oxychloride

\begin{tabular}{|c|c|c|c|c|c|c|c|}
\hline \multicolumn{4}{|c}{ Method A } & \multicolumn{4}{c|}{ Method B } \\
\hline $\begin{array}{c}\text { Time } \\
(\mathrm{min})\end{array}$ & $\begin{array}{c}\text { Volume of } \\
\text { Gas (mL) }\end{array}$ & $\begin{array}{c}\text { Mass of } \\
\mathrm{HCl}(\mathrm{g})\end{array}$ & $\begin{array}{c}\text { Yield } \\
(\%)\end{array}$ & $\begin{array}{c}\text { Time } \\
(\mathrm{min})\end{array}$ & $\begin{array}{c}\text { Volume of } \\
\text { Gas (mL) }\end{array}$ & $\begin{array}{c}\text { Mass of } \\
\mathrm{HCl}(\mathrm{g})\end{array}$ & $\begin{array}{c}\text { Yield } \\
(\%)\end{array}$ \\
\hline & & & & 0.33 & 13.6 & 0.0310 & 28.3 \\
\hline 0.5 & 6.2 & 0.0141 & 13 & 0.5 & 12.5 & 0.0285 & 26.0 \\
\hline 1 & 7.3 & 0.0166 & 15 & 1 & 10.6 & 0.0242 & 22.1 \\
\hline 2 & 7.8 & 0.0178 & 16 & 2 & 5.1 & 0.0116 & 11 \\
\hline 3 & 8.2 & 0.0187 & 17 & 3 & 3.7 & 0.0084 & 7.7 \\
\hline & & & & 4 & 2.9 & 0.0066 & 6.0 \\
\hline 5 & 8.9 & 0.0203 & 19 & 5 & 2.4 & 0.0055 & 5.0 \\
\hline 10 & 9.5 & 0.0216 & 20 & 10 & 1.8 & 0.0041 & 3.8 \\
\hline 20 & 10.7 & 0.0244 & 22 & 20 & 1.7 & 0.0039 & 3.5 \\
\hline 30 & 11.6 & 0.0264 & 24 & & & & \\
\hline 60 & 12.7 & 0.0289 & 26 & & & & \\
\hline 120 & 13.3 & 0.0303 & 28 & & & & \\
\hline 180 & 13.8 & 0.0314 & 29 & & & & \\
\hline
\end{tabular}

Comments: The 1999 experiments were repeated in 2003 to obtain more data on the early stages of the reaction. In method A, about one-sixth of the $\mathrm{HCl}$ evolved as a gas in the first $2 \mathrm{~min}$. Then the rate of evolution slowed dramatically. This suggested that hydrolysis of the first $\mathrm{Cl}$ atom according to the equation 


$$
\mathrm{OPCl}_{3}+\mathrm{H}_{2} \mathrm{O} \longrightarrow \mathrm{HCl}+\mathrm{OPCl}_{2} \mathrm{OH}
$$

was substantially faster than hydrolysis of the second and third. In method $\mathrm{B}, \mathrm{HCl}(\mathrm{g})$ formed rapidly and then dissolved quickly in the excess water [as indicated by the quick maxima in the $\mathrm{HCl}(\mathrm{g})$ yield curves]. In a spill into open water, air currents across the water's surface would sweep $\mathrm{HCl}(\mathrm{g})$ away and so reduce such dissolution.

\section{UN 1816 - Propyltrichlorosilane, $\mathrm{CH}_{3} \mathrm{CH}_{2} \mathrm{CH}_{2} \mathrm{SiCl}_{3}$}

Propyltrichlorosilane is a liquid with a relative molar mass of 177.53 and a roomtemperature density of $1.195 \mathrm{~g} / \mathrm{cm}^{3}$. In method A, $1.00 \mathrm{mmol}$ of it was mixed with $1.50 \mathrm{mmol}$ of water. In method B, $1.00 \mathrm{mmol}$ of it was mixed with $7.50 \mathrm{mmol}$ of water. Reaction according to the equation

$$
\mathrm{CH}_{3}\left(\mathrm{CH}_{2}\right)_{2} \mathrm{SiCl}_{3}+3 / 2 \mathrm{H}_{2} \mathrm{O} \longrightarrow 3 \mathrm{HCl}+\left[\mathrm{CH}_{3}\left(\mathrm{CH}_{2}\right)_{2} \mathrm{SiO}_{3 / 2}\right]_{\mathrm{n}} \text { polymer }
$$

was taken as the source of $\mathrm{HCl}(\mathrm{g})$. The theoretical yield of $\mathrm{HCl}(\mathrm{g})(3.00 \mathrm{mmol})$ corresponded to $48.0 \mathrm{~mL}$ of gas in the calibrated experimental apparatus.

2003 Experimental Results for Propyltrichlorosilane

\begin{tabular}{|c|c|c|c|c|c|c|c|}
\hline \multicolumn{3}{|c|}{ Method A } & \multicolumn{4}{c|}{ Method B } \\
\hline $\begin{array}{c}\text { Time } \\
(\mathrm{min})\end{array}$ & $\begin{array}{c}\text { Volume of } \\
\text { Gas (mL) }\end{array}$ & $\begin{array}{c}\text { Mass of } \\
\mathrm{HCl}(\mathrm{g})\end{array}$ & $\begin{array}{c}\text { Yield } \\
(\%)\end{array}$ & $\begin{array}{c}\text { Time } \\
(\mathrm{min})\end{array}$ & $\begin{array}{c}\text { Volume of } \\
\text { Gas (mL) }\end{array}$ & $\begin{array}{c}\text { Mass of } \\
\mathrm{HCl}(\mathrm{g})\end{array}$ & $\begin{array}{c}\text { Yield } \\
(\%)\end{array}$ \\
\hline 1 & 2.0 & 0.0046 & 4.2 & 1 & 7.2 & 0.016 & 15.0 \\
\hline 2 & 4.8 & 0.0109 & 10.0 & 2 & 12.6 & 0.029 & 26.3 \\
\hline 3 & 8.2 & 0.0187 & 17.1 & 3 & 15.7 & 0.036 & 32.7 \\
\hline 4 & 12.2 & 0.0278 & 25.4 & 4 & 18.0 & 0.041 & 37.5 \\
\hline 5 & 15.4 & 0.0351 & 32.1 & 5 & 19.1 & 0.044 & 39.8 \\
\hline 6 & 17.8 & 0.0406 & 37.1 & 6 & 20.0 & 0.046 & 41.7 \\
\hline 7 & 20.3 & 0.0463 & 42.3 & 7 & 20.4 & 0.046 & 42.5 \\
\hline 8 & 22.4 & 0.0511 & 46.7 & 8 & 20.6 & 0.047 & 42.9 \\
\hline 9 & 24.2 & 0.0552 & 50.4 & 9 & 20.7 & 0.047 & 43.1 \\
\hline 10 & 25.8 & 0.0588 & 53.8 & 10 & 20.7 & 0.047 & 43.1 \\
\hline 11 & 27.6 & 0.0629 & 57.5 & 15 & 20.4 & 0.046 & 42.5 \\
\hline 12 & 29.7 & 0.0677 & 61.9 & 20 & 19.9 & 0.045 & 41.5 \\
\hline 13 & 32.8 & 0.0748 & 68.3 & 25 & 19.3 & 0.044 & 40.2 \\
\hline 14 & 36.6 & 0.0834 & 76.3 & 30 & 18.7 & 0.043 & 39.0 \\
\hline 15 & 39.1 & 0.0891 & 81.5 & 40 & 18.1 & 0.041 & 37.7 \\
\hline 16 & 40.5 & 0.0923 & 84.4 & 50 & 17.7 & 0.040 & 36.9 \\
\hline 18 & 42.8 & 0.0975 & 89.2 & 60 & 17.3 & 0.039 & 36.0 \\
\hline 20 & 44.1 & 0.1005 & 91.9 & 90 & 17.0 & 0.039 & 35.4 \\
\hline 25 & 44.4 & 0.1012 & 92.5 & & & & \\
\hline 30 & 44.5 & 0.1014 & 92.7 & & & & \\
\hline
\end{tabular}


Comments: In method A, the reaction formed a white solid polymeric by-product. Despite this, the system eventually produced nearly $93 \%$ of the theoretical amount of $\mathrm{HCl}(\mathrm{g})$. In method $\mathrm{B}$, the smaller yield of $\mathrm{HCl}(\mathrm{g})$ was ascribed to (1) sequestration of some monomer by the polysiloxane product and (2) dissolution of $\mathrm{HCl}(\mathrm{g})$ in the excess water to produce $\mathrm{HCl}(\mathrm{aq})$.

\section{UN 1818 - Tetrachlorosilane, $\mathrm{SiCl}_{4}$}

Tetrachlorosilane, a liquid, has a relative molar mass of 169.90 and a room-temperature density of $1.483 \mathrm{~g} / \mathrm{cm}^{3}$. Reaction according to the equation

$$
\mathrm{SiCl}_{4}+2 \mathrm{H}_{2} \mathrm{O} \longrightarrow 4 \mathrm{HCl}+\left(\mathrm{SiO}_{2}\right)_{\mathrm{n}} \text { polymer }
$$

was taken as the only source of evolved gas. In all experiments, $1.00 \mathrm{mmol}(0.170 \mathrm{~g})$ of $\mathrm{SiCl}_{4}$ was used, so the theoretical yield of $\mathrm{HCl}(\mathrm{g})$ was $4.00 \mathrm{mmol}(0.146 \mathrm{~g})$. This amount of gas corresponded to $64.0 \mathrm{~mL}$ of gas in the calibrated experimental apparatus.

1999 Experimental Results for Tetrachlorosilane

\begin{tabular}{|c|c|c|c|c|c|c|c|}
\hline \multicolumn{4}{|c|}{ Method A } & \multicolumn{4}{c|}{ Method B } \\
\hline $\begin{array}{c}\text { Time } \\
(\mathrm{min})\end{array}$ & $\begin{array}{c}\text { Volume of } \\
\text { Gas (mL) }\end{array}$ & $\begin{array}{c}\text { Mass of } \\
\mathrm{HCl}(\mathrm{g})\end{array}$ & $\begin{array}{c}\text { Yield } \\
(\%)\end{array}$ & $\begin{array}{c}\text { Time } \\
(\mathrm{min})\end{array}$ & $\begin{array}{c}\text { Volume of } \\
\text { Gas (mL) }\end{array}$ & $\begin{array}{c}\text { Mass of } \\
\mathrm{HCl}(\mathrm{g})\end{array}$ & $\begin{array}{c}\text { Yield } \\
(\%)\end{array}$ \\
\hline 1 & 15 & 0.034 & 23 & 1 & 20 & 0.046 & 31 \\
\hline 5 & 18 & 0.041 & 28 & 5 & 22 & 0.050 & 34 \\
\hline 10 & 19 & 0.043 & 30 & 10 & 21 & 0.048 & 33 \\
\hline 20 & 20 & 0.046 & 31 & 20 & 19 & 0.043 & 30 \\
\hline
\end{tabular}

2003 Experimental Results for Tetrachlorosilane

\begin{tabular}{|c|c|c|c|c|c|c|c|}
\hline \multicolumn{4}{|c|}{ Method A } & \multicolumn{4}{c|}{ Method B } \\
\hline $\begin{array}{c}\text { Time } \\
(\mathrm{min})\end{array}$ & $\begin{array}{c}\text { Volume of } \\
\text { Gas }(\mathrm{mL})\end{array}$ & $\begin{array}{c}\text { Mass of } \\
\mathrm{HCl}(\mathrm{g})\end{array}$ & $\begin{array}{c}\text { Yield } \\
(\%)\end{array}$ & $\begin{array}{c}\text { Time } \\
(\mathrm{min})\end{array}$ & $\begin{array}{c}\text { Volume of } \\
\text { Gas (mL) }\end{array}$ & $\begin{array}{c}\text { Mass of } \\
\mathrm{HCl}(\mathrm{g})\end{array}$ & $\begin{array}{c}\text { Yield } \\
(\%)\end{array}$ \\
\hline 0.5 & 10.1 & 0.0230 & 15.8 & 0.5 & 14.7 & 0.0335 & 23.0 \\
\hline 1 & 14.7 & 0.0335 & 23.0 & 1 & 20.9 & 0.048 & 32.7 \\
\hline 2 & 16.4 & 0.0374 & 25.6 & 2 & 21.5 & 0.049 & 33.6 \\
\hline 3 & 17.2 & 0.0392 & 26.9 & 3 & 22.3 & 0.051 & 34.8 \\
\hline 4 & 17.8 & 0.0406 & 27.8 & 4 & 22.8 & 0.052 & 35.6 \\
\hline 5 & 18.2 & 0.0415 & 28.4 & 5 & 23.1 & 0.053 & 36.1 \\
\hline 7 & 18.8 & 0.0428 & 29.4 & 7 & 22.9 & 0.052 & 35.8 \\
\hline 10 & 19.3 & 0.0440 & 30.2 & 10 & 22.1 & 0.050 & 34.5 \\
\hline 20 & 20.5 & 0.0467 & 32.0 & 20 & 19.8 & 0.045 & 30.9 \\
\hline 30 & 20.8 & 0.0474 & 32.5 & 30 & 19.4 & 0.044 & 30.3 \\
\hline 45 & 21.1 & 0.0481 & 33.0 & 45 & 18.8 & 0.043 & 29.4 \\
\hline 60 & 21.3 & 0.0485 & 33.3 & 60 & 18.4 & 0.042 & 28.8 \\
\hline
\end{tabular}


Comments: The 1999 experiments were repeated in 2003 to obtain more information on the early course of the reaction. A white solid formed immediately upon mixing the reactants. This siliceous by-product almost certainly sequestered water, preventing productive contact between the reactants [its probable formula was $\left(\mathrm{SiO}_{2}\right)_{\mathrm{n}} \cdot \mathrm{xH}_{2} \mathrm{O}$ ]. The yield-versus-time curves for $\mathrm{HCl}(\mathrm{g})$ in method $\mathrm{B}$ had maxima. This indicated that the rate of dissolution of $\mathrm{HCl}(\mathrm{g})$ in the excess water exceeded its rate of production toward the end of the experiment. The yield of $\mathrm{HCl}(\mathrm{g})$ in the two method B runs settled to a final average of $29.4 \%$, or $1.18 \mathrm{mmol}$. If the hydrolysis of the test substance was complete at this point, then $8.00 \mathrm{mmol}$ of water was present and $(4.00-1.18) \mathrm{mmol}$ of $\mathrm{HCl}$ was dissolved in it. The mole fraction of $\mathrm{HCl}$ in this solution was

$$
X_{\mathrm{HCl}}=\frac{n_{\mathrm{HCl}}}{n_{\mathrm{HCl}}+n_{\mathrm{H}_{2} \mathrm{O}}}=\frac{(4.00-1.18)}{(4.00-1.18)+8.00}=0.26
$$

This value is quite close to the literature value for $X_{\mathrm{HCl}}$ in saturated aqueous solutions of $\mathrm{HCl}$ under the conditions of the experiments (see Section C.1.2).

\section{UN 1827 - Tin Tetrachloride, $\mathrm{SnCl}_{4}$}

Tin tetrachloride, also called stannic chloride, is a liquid with a relative molar mass of 266.6 and a room-temperature density of $2.28 \mathrm{~g} / \mathrm{cm}^{3}$. In method A, $1.00 \mathrm{mmol}$ of the substance was mixed with $4.00 \mathrm{mmol}$ of water. In method B, $1.00 \mathrm{mmol}$ was mixed with $20.0 \mathrm{mmol}$ of water. Reaction according to the equation

$$
\mathrm{SnCl}_{4}+4 \mathrm{H}_{2} \mathrm{O} \longrightarrow 4 \mathrm{HCl}+\mathrm{SnO}_{2} \cdot 2 \mathrm{H}_{2} \mathrm{O}
$$

was used for computations of mass and yield. The theoretical yield of $\mathrm{HCl}(\mathrm{g})$ was $4.00 \mathrm{mmol}$, which corresponded to $64.0 \mathrm{~mL}$ of gas in the calibrated experimental apparatus.

1999 Experimental Results for Tin Tetrachloride

\begin{tabular}{|c|c|c|c|c|c|c|c|}
\hline \multicolumn{3}{|c|}{ Method A } & \multicolumn{4}{c|}{ Method B } \\
\hline $\begin{array}{c}\text { Time } \\
(\mathrm{min})\end{array}$ & $\begin{array}{c}\text { Volume of } \\
\text { Gas (mL) }\end{array}$ & $\begin{array}{c}\text { Mass of } \\
\mathrm{HCl}(\mathrm{g})\end{array}$ & $\begin{array}{c}\text { Yield } \\
(\%)\end{array}$ & $\begin{array}{c}\text { Time } \\
(\mathrm{min})\end{array}$ & $\begin{array}{c}\text { Volume of } \\
\text { Gas (mL) }\end{array}$ & $\begin{array}{c}\text { Mass of } \\
\mathrm{HCl}(\mathrm{g})\end{array}$ & $\begin{array}{c}\text { Yield } \\
(\%)\end{array}$ \\
\hline 1 & 1 & 0.00057 & 1.6 & 1 & 1 & 0.00057 & 1.6 \\
\hline 5 & 1 & 0.00057 & 1.6 & 5 & 0.5 & 0.0028 & 1 \\
\hline 10 & 1 & 0.00057 & 1.6 & 10 & 0 & 0 & 0 \\
\hline 20 & 1 & 0.00057 & 1.6 & 20 & 0 & 0 & 0 \\
\hline
\end{tabular}

Comments: Less than $2 \%$ of the theoretical amount of $\mathrm{HCl}(\mathrm{g})$ was generated. 


\section{UN 1831 — Oleum (fuming sulfuric acid)}

Oleum, a liquid, has an approximate relative molar mass of 122. In method A, $1.0 \mathrm{mmol}$ of it was mixed with $1.00 \mathrm{mmol}$ of water. In method B, $1.0 \mathrm{mmol}$ was mixed with $5.00 \mathrm{mmol}$ of water. No equation was proposed for a reaction generating a TIH gas beyond the following

$$
\mathrm{H}_{2} \mathrm{SO}_{4} \cdot\left(\mathrm{SO}_{3}\right)_{\mathrm{n}}+\mathrm{n} \mathrm{H}_{2} \mathrm{O} \longrightarrow \mathrm{H}_{2} \mathrm{SO}_{4}
$$

1999 Experimental Results for Oleum

\begin{tabular}{|c|c|c|c|c|c|c|c|}
\hline \multicolumn{4}{|c|}{ Method A } & \multicolumn{4}{c|}{ Method B } \\
\hline $\begin{array}{c}\text { Time } \\
(\mathrm{min})\end{array}$ & $\begin{array}{c}\text { Volume of } \\
\text { Gas (mL) }\end{array}$ & $\begin{array}{c}\text { Mass of } \\
\text { Gas (g) }\end{array}$ & $\begin{array}{c}\text { Yield } \\
(\%)\end{array}$ & $\begin{array}{c}\text { Time } \\
(\mathrm{min})\end{array}$ & $\begin{array}{c}\text { Volume of } \\
\text { Gas (mL) }\end{array}$ & $\begin{array}{c}\text { Mass of } \\
\text { Gas (g) }\end{array}$ & $\begin{array}{c}\text { Yield } \\
(\%)\end{array}$ \\
\hline 1 & 1 & - & - & 1 & 1 & - & - \\
\hline 5 & 1 & - & - & 5 & 1 & - & - \\
\hline 10 & 0.5 & - & - & 10 & 0.5 & - & - \\
\hline 20 & 0.5 & - & - & 20 & 0.5 & - & - \\
\hline
\end{tabular}

Comments: Little evidence of a gaseous product was detected. The dissolution reaction in water produced a large amount of heat, which probably caused the slight volume change (on the order of $1 \mathrm{~mL}$ ). The $\mathrm{TIH}$ concern associated with this material was the production of an acidic mist. However, neither method A nor B simulated conditions that might generate an acidic mist.

\section{UN 1834 - Sulfuryl Chloride, $\mathrm{SO}_{2} \mathrm{Cl}_{2}$}

Sulfuryl chloride is a liquid with a relative molar mass of 134.96 and a room-temperature density of $1.67 \mathrm{~g} / \mathrm{cm}^{3}$. In method $\mathrm{A}, 2.00 \mathrm{mmol}$ of water was added to $1.00 \mathrm{mmol}$ of it. In method B, $1.00 \mathrm{mmol}$ of it was mixed with $10.0 \mathrm{mmol}$ of water. The reaction

$$
\mathrm{SO}_{2} \mathrm{Cl}_{2}+2 \mathrm{H}_{2} \mathrm{O} \longrightarrow 2 \mathrm{HCl}+\mathrm{H}_{2} \mathrm{SO}_{4}
$$

was taken as the source of evolved gas. Generation of $2.00 \mathrm{mmol}$ of gas corresponded to an increase in volume of $32.0 \mathrm{~mL}$ in the calibrated apparatus. 
1999 Experimental Results for Sulfuryl Chloride

\begin{tabular}{|c|c|c|c|c|c|c|c|}
\hline \multicolumn{4}{|c|}{ Method A } & \multicolumn{4}{c|}{ Method B } \\
\hline $\begin{array}{c}\text { Time } \\
(\mathrm{min})\end{array}$ & $\begin{array}{c}\text { Volume of } \\
\text { Gas (mL) }\end{array}$ & $\begin{array}{c}\text { Mass of } \\
\mathrm{HCl}(\mathrm{g})\end{array}$ & $\begin{array}{c}\text { Yield } \\
(\%)\end{array}$ & $\begin{array}{c}\text { Time } \\
(\mathrm{min})\end{array}$ & $\begin{array}{c}\text { Volume of } \\
\text { Gas (mL) }\end{array}$ & $\begin{array}{c}\text { Mass of } \\
\mathrm{HCl}(\mathrm{g})\end{array}$ & $\begin{array}{c}\text { Yield } \\
(\%)\end{array}$ \\
\hline 1 & 3 & 0.0068 & 9 & 1 & 4 & 0.0091 & 12 \\
\hline 2 & 5 & 0.0114 & 16 & 2 & 5 & 0.0114 & 16 \\
\hline 3 & 6.5 & 0.015 & 20 & 3 & 5.5 & 0.0125 & 17 \\
\hline 5 & 10 & 0.023 & 31 & 5 & 6 & 0.0137 & 19 \\
\hline 10 & 18 & 0.041 & 56 & 10 & 6.5 & 0.0148 & 20 \\
\hline 15 & 24 & 0.055 & 75 & 15 & 5.5 & 0.0125 & 17 \\
\hline 20 & 26 & 0.059 & 81 & 20 & 5 & 0.0114 & 16 \\
\hline 30 & 28 & 0.064 & 88 & 30 & 5 & 0.0114 & 16 \\
\hline 60 & 30 & 0.068 & 94 & & & & \\
\hline 90 & 31 & 0.071 & 97 & & & & \\
\hline 120 & 31 & 0.071 & 97 & & & & \\
\hline
\end{tabular}

Comments: In method $\mathrm{A}$, essentially the stoichiometric amount of gaseous $\mathrm{HCl}$ was generated, but slowly. In method $\mathrm{B}$, some $\mathrm{HCl}$ dissolved in the excess water after it was generated. The acidic by-product $\mathrm{H}_{2} \mathrm{SO}_{4}$ that was co-dissolved in the excess water certainty reduced the amount of $\mathrm{HCl}$ it could dissolve and so raised the yield of $\mathrm{HCl}(\mathrm{g})$. In a real spill, the by-product $\mathrm{H}_{2} \mathrm{SO}_{4}$ would be diluted and the amount of $\mathrm{HCl}(\mathrm{g})$ would be less.

\section{UN 1836 - Thionyl Chloride, $\mathrm{SOCl}_{2}$}

Thionyl chloride, a liquid, has a relative molar mass of 118.97 and a room-temperature density of $1.64 \mathrm{~g} / \mathrm{cm}^{3}$. In method A, $1.00 \mathrm{mmol}$ was mixed with $1.00 \mathrm{mmol}$ of water. In method $\mathrm{B}, 1.00 \mathrm{mmol}$ was added to $5.00 \mathrm{mmol}$ of water. The reaction with water generates two TIH gases, hydrogen chloride and sulfur dioxide, according to the equation

$$
\mathrm{SOCl}_{2}+\mathrm{H}_{2} \mathrm{O} \longrightarrow 2 \mathrm{HCl}+\mathrm{SO}_{2}
$$

The theoretical yield of TIH gases is $3.00 \mathrm{mmol}$, which corresponds to $48.0 \mathrm{~mL}$ of mixed gases (32.0 $\mathrm{mL}$ of $\mathrm{HCl}$ and $16.0 \mathrm{~mL}$ of $\mathrm{SO}_{2}$ ) in the calibrated experimental apparatus. 


\begin{tabular}{|c|c|c|c|c|c|}
\hline \multicolumn{6}{|c|}{1999 Experimental Results for Thionyl Chloride, Method A } \\
\hline \multicolumn{6}{|c|}{\begin{tabular}{c|c|c|c|c|c}
$\begin{array}{c}\text { Time } \\
(\mathrm{min})\end{array}$ & $\begin{array}{c}\text { Volume of } \\
\text { Gases }(\mathrm{mL})\end{array}$ & $\begin{array}{c}\text { Mass of } \mathrm{HCl} \\
(\mathrm{g})\end{array}$ & $\begin{array}{c}\text { Yield of } \\
\mathrm{HCl}(\%)\end{array}$ & $\begin{array}{c}\text { Mass of } \\
\mathrm{SO}_{2}(\mathrm{~g})\end{array}$ & $\begin{array}{c}\text { Yield of } \\
\mathrm{SO}_{2}(\%)\end{array}$ \\
\end{tabular}} \\
\hline 1 & 15 & 0.023 & 31 & 0.020 & 31 \\
\hline 2 & 35 & 0.053 & 73 & 0.047 & 73 \\
\hline 3 & 46 & 0.070 & 96 & 0.061 & 96 \\
\hline 5 & 48 & 0.073 & 100 & 0.064 & 100 \\
\hline 10 & 46 & 0.073 & 100 & 0.056 & 87.5 \\
\hline 20 & 45 & 0.073 & 100 & 0.052 & 81 \\
\hline
\end{tabular}

\begin{tabular}{|c|c|c|c|c|c|}
\hline \multicolumn{6}{|c|}{1999 Experimental Results for Thionyl Chloride, Method B } \\
\hline $\begin{array}{l}\text { Time } \\
(\mathrm{min})\end{array}$ & $\begin{array}{l}\text { Volume of } \\
\text { Gases (mL) }\end{array}$ & \begin{tabular}{|c} 
Mass of $\mathrm{HCl}$ \\
$(\mathrm{g})$
\end{tabular} & $\begin{array}{l}\text { Yield of } \\
\mathrm{HCl}(\%)\end{array}$ & $\begin{array}{c}\text { Mass of } \mathrm{SO}_{2} \\
(\mathrm{~g})\end{array}$ & $\begin{array}{l}\text { Yield of } \\
\mathrm{SO}_{2}(\%)\end{array}$ \\
\hline 1 & 33 & \begin{tabular}{|l|}
0.041 \\
\end{tabular} & 56 & \begin{tabular}{|l|}
0.060 \\
\end{tabular} & 94 \\
\hline 2 & 35 & 0.043 & 59 & 0.064 & 100 \\
\hline 3 & 33.5 & 0.040 & 55 & 0.064 & 100 \\
\hline 5 & 32 & 0.039 & 53 & 0.060 & 94 \\
\hline 10 & 30 & 0.039 & 53 & 0.052 & 81 \\
\hline 20 & 29 & 0.039 & 53 & 0.048 & 75 \\
\hline
\end{tabular}

Comments: The reaction generated two $\mathrm{TIH}$ gases, $\mathrm{HCl}$ and $\mathrm{SO}_{2}$, concurrently in a 2:1 molar ratio, but the experiment measured only the total volume of gas, not their separate volumes. In method A, the theoretical yield of the mixed gases appeared within 5 min of mixing. The amounts of $\mathrm{HCl}(\mathrm{g})$ and $\mathrm{SO}_{2}(\mathrm{~g})$ at 1,2 , and 3 min were calculated by assuming that the 2:1 molar ratio held at those points in time (that is, that no differential dissolution of gas occurred before the water was consumed by the reaction). At the end of the reaction some absorption of sulfur dioxide into the manometric fluid (octane saturated with $\mathrm{HCl}$ ) was observed. In method $\mathrm{B}$, the partition into mass of $\mathrm{HCl}(\mathrm{g})$ and mass of $\mathrm{SO}_{2}(\mathrm{~g})$ was estimated by assuming that $40 \%$ of the total $\mathrm{HCl}$ dissolved instantly in the excess water.

\section{UN 1838 - Titanium Tetrachloride, TiCl $_{4}$}

Titanium tetrachloride, a liquid, has a relative molar mass of 189.71 and a roomtemperature density of $1.730 \mathrm{~g} / \mathrm{cm}^{3}$. In method A, $1.00 \mathrm{mmol}$ of the substance was mixed with $2.00 \mathrm{mmol}$ of water. In method B, $1.00 \mathrm{mmol}$ was mixed with $10.0 \mathrm{mmol}$ of water. Reaction according to the equation

$$
\mathrm{TiCl}_{4}+2 \mathrm{H}_{2} \mathrm{O} \longrightarrow 4 \mathrm{HCl}+\mathrm{TiO}_{2}
$$

was taken as the source of $\mathrm{HCl}(\mathrm{g})$. The theoretical yield of $\mathrm{HCl}(\mathrm{g})$ was $4.00 \mathrm{mmol}$, which corresponded to $64.0 \mathrm{~mL}$ of gas in the calibrated experimental apparatus. 
1999 Experimental Results for Titanium Tetrachloride

\begin{tabular}{|c|c|c|c|c|c|c|c|}
\hline \multicolumn{4}{|c|}{ Method A } & \multicolumn{4}{c|}{ Method B } \\
\hline $\begin{array}{c}\text { Time } \\
(\mathrm{min})\end{array}$ & $\begin{array}{c}\text { Volume of } \\
\text { Gas (mL) }\end{array}$ & $\begin{array}{c}\text { Mass of } \\
\mathrm{HCl}(\mathrm{g})\end{array}$ & $\begin{array}{c}\text { Yield } \\
(\%)\end{array}$ & $\begin{array}{c}\text { Time } \\
(\mathrm{min})\end{array}$ & $\begin{array}{c}\text { Volume of } \\
\text { Gas (mL) }\end{array}$ & $\begin{array}{c}\text { Mass of } \\
\mathrm{HCl}(\mathrm{g})\end{array}$ & $\begin{array}{c}\text { Yield } \\
(\%)\end{array}$ \\
\hline 1 & 10 & 0.023 & 16 & 1 & 10 & 0.0182 & 16 \\
\hline 5 & 17 & 0.039 & 27 & 5 & 14 & 0.027 & 8 \\
\hline 10 & 17 & 0.039 & 27 & 10 & 4 & 0.030 & 6 \\
\hline 20 & 17 & 0.039 & 27 & 20 & 4 & 0.030 & 6 \\
\hline
\end{tabular}

2003 Experimental Results for Titanium Tetrachloride

\begin{tabular}{|c|c|c|c|c|c|c|c|}
\hline \multicolumn{4}{|c|}{ Method A } & \multicolumn{4}{c|}{ Method B } \\
\hline $\begin{array}{c}\text { Time } \\
(\mathrm{min})\end{array}$ & $\begin{array}{c}\text { Volume of } \\
\text { Gas (mL) }\end{array}$ & $\begin{array}{c}\text { Mass of } \\
\text { HCl (g) }\end{array}$ & $\begin{array}{c}\text { Yield } \\
(\%)\end{array}$ & $\begin{array}{c}\text { Time } \\
(\mathrm{min})\end{array}$ & $\begin{array}{c}\text { Volume of } \\
\text { Gas }(\mathrm{mL})\end{array}$ & $\begin{array}{c}\text { Mass of } \\
\mathrm{HCl}(\mathrm{g})\end{array}$ & $\begin{array}{c}\text { Yield } \\
(\%)\end{array}$ \\
\hline 0.5 & 7.2 & 0.0164 & 11.3 & 0.5 & 7.4 & 0.0169 & 11.6 \\
\hline 1 & 11.3 & 0.0257 & 17.7 & 1 & 11.2 & 0.0255 & 17.5 \\
\hline 2 & 15.7 & 0.0358 & 24.5 & 2 & 9.2 & 0.0210 & 14.4 \\
\hline 3 & 17.1 & 0.0390 & 26.7 & 3 & 7.1 & 0.0162 & 11.1 \\
\hline 4 & 18.2 & 0.0415 & 28.4 & 4 & 5.7 & 0.0130 & 8.9 \\
\hline 5 & 18.6 & 0.0424 & 29.1 & 5 & 5.1 & 0.0116 & 8.0 \\
\hline 7 & 18.9 & 0.0431 & 29.5 & 7 & 4.7 & 0.0107 & 7.3 \\
\hline 10 & 19.1 & 0.0435 & 29.8 & 10 & 4.2 & 0.0096 & 6.6 \\
\hline 20 & 19.2 & 0.0438 & 30.0 & 20 & 4.0 & 0.0091 & 6.3 \\
\hline 30 & 19.3 & 0.0440 & 30.2 & 30 & 3.8 & 0.0087 & 5.9 \\
\hline 60 & 19.4 & 0.0442 & 30.3 & 60 & 3.7 & 0.0084 & 5.8 \\
\hline
\end{tabular}

Comments: The 1999 experiments were repeated in 2003 to obtain more data on the early course of the reaction. $\mathrm{TiCl}_{4}$ reacts very vigorously with water. In method $\mathrm{A}$, the injected water was immediately covered with a white crust, probably titanium dioxide. This might have prevented complete reaction of water with the remaining $\mathrm{TiCl}_{4}$, despite the stirring. In method $\mathrm{B}$, the yield of the gas peaked and then diminished. Apparently, some $\mathrm{HCl}(\mathrm{g})$ later dissolved in the excess water. In a spill into open water, air currents across the water's surface would probably reduce such dissolution by blowing the $\mathrm{HCl}(\mathrm{g})$ away.

According to the literature, ${ }^{4}$ mixing $\mathrm{TiCl}_{4}$ and a 50 -fold excess of $\mathrm{H}_{2} \mathrm{O}$ gives an immediate voluminous precipitate of $\mathrm{TiO}_{2} \bullet \times\left(\mathrm{H}_{2} \mathrm{O}\right)$, but the yield of $\mathrm{HCl}(\mathrm{aq})$ is substantially less than the amount calculated on the basis of the preceding chemical equation.

4 Information obtained from an on-line commercial database, "Gmelin," through a multi-database portal provided by CAS. Additional information can be obtained at http://www.cas.org/ONLINE/DBSS/gmelinss.html. 


\section{UN 1939 - Phosphorus Oxybromide, $\mathrm{OPBr}_{3}$}

Phosphorus oxybromide, also called phosphoryl bromide, is a solid with a relative molar mass of 286.70. In method A, $3.00 \mathrm{mmol}$ of water was added to $1.00 \mathrm{mmol}$ of it. In method B, $1.00 \mathrm{mmol}$ of it was added to $15.0 \mathrm{mmol}$ of water. The reaction

$$
\mathrm{OPBr}_{3}+3 \mathrm{H}_{2} \mathrm{O} \longrightarrow 3 \mathrm{HBr}+\mathrm{OP}(\mathrm{OH})_{3}
$$

was taken as the source of evolved gas. Generation of $3.00 \mathrm{mmol}$ of gas corresponded to an increase in volume of $48.0 \mathrm{~mL}$ in the calibrated apparatus.

\section{Experimental Data for Phosphorus Oxybromide}

\begin{tabular}{|c|c|c|c|c|c|c|c|}
\hline \multicolumn{4}{|c|}{ Method A } & \multicolumn{4}{c|}{ Method B } \\
\hline $\begin{array}{c}\text { Time } \\
(\mathrm{min})\end{array}$ & $\begin{array}{c}\text { Volume of } \\
\text { Gas (mL) }\end{array}$ & $\begin{array}{c}\text { Mass of } \\
\mathrm{HBr}(\mathrm{g})\end{array}$ & $\begin{array}{c}\text { Yield } \\
(\%)\end{array}$ & $\begin{array}{c}\text { Time } \\
(\mathrm{min})\end{array}$ & $\begin{array}{c}\text { Volume of } \\
\text { Gas (mL) }\end{array}$ & $\begin{array}{c}\text { Mass of } \\
\mathrm{HBr}(\mathrm{g})\end{array}$ & $\begin{array}{c}\text { Yield } \\
(\%)\end{array}$ \\
\hline 3 & 0.9 & 0.0046 & 1.9 & 1 & 0.0 & 0.00 & 0.0 \\
\hline 5 & 1.5 & 0.0076 & 3.1 & 2 & 0.0 & 0.00 & 0.0 \\
\hline 10 & 2.3 & 0.0116 & 4.8 & 3 & 0.0 & 0.00 & 0.0 \\
\hline 15 & 3.4 & 0.0172 & 7.1 & 5 & 0.0 & 0.00 & 0.0 \\
\hline 20 & 4.4 & 0.0223 & 9.2 & 10 & 0.0 & 0.00 & 0.0 \\
\hline 30 & 5.7 & 0.0288 & 11.9 & 15 & 0.0 & 0.00 & 0.0 \\
\hline 40 & 6.8 & 0.0344 & 14.2 & 20 & 0.0 & 0.00 & 0.0 \\
\hline 50 & 7.6 & 0.038 & 15.8 & 30 & & 0.00 & 0.0 \\
\hline 60 & 8.4 & 0.042 & 17.5 & & & & \\
\hline 90 & 9.8 & 0.050 & 20.4 & & & & \\
\hline 120 & 11.4 & 0.058 & 23.8 & & & & \\
\hline 180 & 13.0 & 0.066 & 27.0 & & & & \\
\hline 240 & 13.3 & 0.067 & 27.7 & & & & \\
\hline
\end{tabular}

Comments: In method A, somewhat less than one-third of the theoretical amount of $\mathrm{HBr}$ evolved slowly. This suggests little hydrolysis of the second and third $\mathrm{Br}$ atoms. Because the amount of water used in the experiment was $3.00 \mathrm{mmol}$, water was effectively in excess and some $\mathrm{HBr}(\mathrm{g})$ dissolved in it. In method $\mathrm{B}$, there was evidence of reaction, but the $\mathrm{HBr}$ evidently dissolved immediately in the excess water.

It was interesting to compare the hydrolysis of this compound to the hydrolysis of phosphorus oxychloride (UN 1810). In method A, the ultimate yields of $\mathrm{HBr}(\mathrm{g})$ and $\mathrm{HCl}(\mathrm{g})$ were nearly identical but $\operatorname{HBr}(\mathrm{g})$ formed much more slowly. The hydrolysis of phosphorus oxychloride in method $\mathrm{B}$ generated $\mathrm{HCl}(\mathrm{g})$ that subsequently mostly dissolved in the excess water. This contrasts to the hydrolysis of phosphorus oxybromide in method $\mathrm{B}$, where the dissolution of $\mathrm{HBr}$ in the excess water was rapid enough, relative to the rate of formation of $\mathrm{HBr}$, to prevent evolution of $\operatorname{HBr}(\mathrm{g})$. 


\section{UN 2308 - Nitrosylsulfuric Acid, $\mathrm{HO}_{3} \mathrm{SONO}$}

Pure nitrosylsulfuric acid is a solid with a relative molar mass of 127.07. In method A, $1.00 \mathrm{mmol}$ of water was added to $1.00 \mathrm{mmol}$ of it. In method B, $1.00 \mathrm{mmol}$ of it was added to $5.00 \mathrm{mmol}$ of water. The generation of the TIH gas nitrogen dioxide $\left(\mathrm{NO}_{2}\right)$ was modeled by the equations:

$$
\begin{aligned}
& 3 \mathrm{HO}_{3} \mathrm{SONO}+3 \mathrm{H}_{2} \mathrm{O} \longrightarrow 2 \mathrm{H}_{2} \mathrm{SO}_{4}+3 \mathrm{HONO} \\
& 3 \mathrm{HONO} 2 \mathrm{NO}+\mathrm{HNO}_{3}+\mathrm{H}_{2} \mathrm{O} \\
& 2 \mathrm{NO}+\mathrm{O}_{2} \longrightarrow 2 \mathrm{NO}_{2}
\end{aligned}
$$

The sum of these equations is

$$
3 \mathrm{HO}_{3} \mathrm{SONO}+3 \mathrm{H}_{2} \mathrm{O} \longrightarrow 3 \mathrm{H}_{2} \mathrm{SO}_{4}+\mathrm{HNO}_{3}+\mathrm{H}_{2} \mathrm{O}+2 \mathrm{NO}_{2}
$$

Because the formation of $\mathrm{NO}_{2}$ requires $\mathrm{O}_{2}$, the nitrogen purge was omitted in the experiments.

1999 Experimental Results for Nitrosylsulfuric Acid

\begin{tabular}{|c|c|c|c|c|c|c|c|}
\hline \multicolumn{4}{|c|}{ Method A } & \multicolumn{4}{c|}{ Method B } \\
\hline $\begin{array}{c}\text { Time } \\
(\mathrm{min})\end{array}$ & $\begin{array}{c}\text { Volume of } \\
\text { Gas }(\mathrm{mL})\end{array}$ & $\begin{array}{c}\text { Mass of } \\
\mathrm{NO}_{2}(\mathrm{~g})\end{array}$ & $\begin{array}{c}\text { Yield } \\
(\%)\end{array}$ & $\begin{array}{c}\text { Time } \\
(\mathrm{min})\end{array}$ & $\begin{array}{c}\text { Volume of } \\
\text { Gas (mL) }\end{array}$ & $\begin{array}{c}\text { Mass of } \mathrm{NO}_{2} \\
(\mathrm{~g})\end{array}$ & $\begin{array}{c}\text { Yield } \\
(\%)\end{array}$ \\
\hline 1 & 3 & 0.0086 & 28 & 1 & 11 & 0.0316 & 103 \\
\hline 5 & 1 & 0.0028 & 9.1 & 5 & 10.5 & 0.0302 & 98 \\
\hline 10 & 0.5 & 0.0014 & 4.5 & 10 & 9.5 & 0.0273 & 89 \\
\hline 20 & 0 & 0 & 0 & 20 & 8.5 & 0.0244 & 79 \\
\hline
\end{tabular}

Comments: According to the chemical equations, $1 \mathrm{mmol}$ of $\mathrm{O}_{2}$ was consumed for every $2 \mathrm{mmol}$ of $\mathrm{NO}_{2}$ that was generated. For this reason, the true yield of $\mathrm{NO}_{2}(\mathrm{~g})$ exceeded the amount indicated by the observed changes in volume by a factor of $3 / 2$. The chemical equations also indicated that the theoretical yield of $\mathrm{NO}_{2}$ from $1.00 \mathrm{mmol}$ of nitrosylsulfuric acid is $0.667 \mathrm{mmol}(0.0307 \mathrm{~g})$. These factors were taken into account in computing the masses and yields in the table.

The formation of red $\mathrm{NO}_{2}(\mathrm{~g})$ was observed immediately in both experiments. In method $\mathrm{A}$, the amount of $\mathrm{NO}_{2}(\mathrm{~g})$ diminished after the first minute as the gas dissolved in the byproduct water or otherwise interacted with the other products. The drop in the amount of $\mathrm{NO}_{2}(\mathrm{~g})$ after the first minute in method $\mathrm{B}$ had a similar cause. The amount of $\mathrm{NO}_{2}$ produced at $1 \mathrm{~min}$ in method B slightly exceeded the theoretical maximum, possibly because of side-reactions not represented above. 


\section{UN 2437 - Methylphenyldichlorosilane, $\mathrm{CH}_{3}\left(\mathrm{C}_{6} \mathrm{H}_{5}\right) \mathrm{SiCl}_{2}$}

Methylphenyldichlorosilane is a liquid with relative molar mass of 191.13 and a roomtemperature density of $1.176 \mathrm{~g} / \mathrm{cm}^{3}$. In method A, $1.00 \mathrm{mmol}$ of it was mixed with $1.00 \mathrm{mmol}$ of water. In method B, $1.00 \mathrm{mmol}$ of it was mixed with $5.00 \mathrm{mmol}$ of water. Reaction according to the equation

$$
\mathrm{CH}_{3}\left(\mathrm{C}_{6} \mathrm{H}_{5}\right) \mathrm{SiCl}_{2}+\mathrm{H}_{2} \mathrm{O} \longrightarrow 2 \mathrm{HCl}+\left[\left(\mathrm{CH}_{3}\right)\left(\mathrm{C}_{6} \mathrm{H}_{5}\right) \mathrm{SiO}\right]_{\mathrm{n}} \text { polymer }
$$

was taken as the source of $\mathrm{HCl}(\mathrm{g})$. The theoretical yield of $\mathrm{HCl}(\mathrm{g})$ was $2.00 \mathrm{mmol}$, which corresponded to $32.0 \mathrm{~mL}$ of gas in the calibrated experimental apparatus.

\section{Experimental Data for Methylphenyldichlorosilane}

\begin{tabular}{|c|c|c|c|c|c|c|c|}
\hline \multicolumn{4}{|c|}{ Method A } & \multicolumn{4}{c|}{ Method B } \\
\hline $\begin{array}{c}\text { Time } \\
(\mathrm{min})\end{array}$ & $\begin{array}{c}\text { Volume of } \\
\text { Gas }(\mathrm{mL})\end{array}$ & $\begin{array}{c}\text { Mass of } \\
\mathrm{HCl}(\mathrm{g})\end{array}$ & $\begin{array}{c}\text { Yield } \\
(\%)\end{array}$ & $\begin{array}{c}\text { Time } \\
(\mathrm{min})\end{array}$ & $\begin{array}{c}\text { Volume of } \\
\text { Gas }(\mathrm{mL})\end{array}$ & $\begin{array}{c}\text { Mass of } \\
\mathrm{HCl}(\mathrm{g})\end{array}$ & $\begin{array}{c}\text { Yield } \\
(\%)\end{array}$ \\
\hline 2 & 0.5 & 0.0011 & 1.6 & 2 & 0.6 & 0.038 & 1.9 \\
\hline 5 & 1.3 & 0.0030 & 4.1 & 5 & 1.0 & 0.063 & 3.1 \\
\hline 10 & 2.3 & 0.0052 & 7.2 & 10 & 1.6 & 0.100 & 5.0 \\
\hline 15 & 3.2 & 0.0073 & 10.0 & 15 & 1.8 & 0.113 & 5.6 \\
\hline 20 & 4.0 & 0.0091 & 12.5 & 20 & 2.0 & 0.125 & 6.3 \\
\hline 25 & 4.7 & 0.0107 & 14.7 & 30 & 2.1 & 0.131 & 6.6 \\
\hline 30 & 5.3 & 0.0121 & 16.6 & 45 & 2.2 & 0.138 & 6.9 \\
\hline 40 & 6.2 & 0.0141 & 19.4 & 60 & 2.3 & 0.144 & 7.2 \\
\hline 50 & 6.9 & 0.0157 & 21.6 & 90 & 2.3 & 0.144 & 7.2 \\
\hline 60 & 7.3 & 0.0166 & 22.8 & & & & \\
\hline 90 & 7.8 & 0.0178 & 24.4 & & & & \\
\hline 120 & 8.1 & 0.0185 & 25.3 & & & & \\
\hline 150 & 8.2 & 0.0187 & 25.6 & & & & \\
\hline
\end{tabular}

Comments: In method A, the reaction slowly formed a very viscous liquid polysiloxane that sequestered a considerable proportion of the water. In method B, the liquid polysiloxane also formed. The fact that polymer was a liquid probably aided to the smooth dissolution of the $\mathrm{HCl}(\mathrm{g})$ in the excess water. No maximum was observed on the $\mathrm{HCl}(\mathrm{g})$ yield curve. The reaction strongly resembled that of diethyldichlorosilane (UN 1767) in its rate and yield and in the appearance of polysiloxane in the by-product.

\section{UN 2442 -Trichloroacetyl Chloride, $\mathrm{Cl}_{3} \mathrm{CCOCl}$}

Trichloroacetyl chloride is a liquid with relative molar mass of 181.83 and a roomtemperature density of $1.629 \mathrm{~g} / \mathrm{cm}^{3}$. In method A, $1.00 \mathrm{mmol}$ of it was mixed with $1.00 \mathrm{mmol}$ of water. In method B, $1.00 \mathrm{mmol}$ of it was mixed with $5.00 \mathrm{mmol}$ of water. Reaction according to the equation 


$$
\mathrm{Cl}_{3} \mathrm{CCOCl}+\mathrm{H}_{2} \mathrm{O} \longrightarrow \mathrm{HCl}+\mathrm{Cl}_{3} \mathrm{CCOOH}
$$

was taken as the source of $\mathrm{HCl}(\mathrm{g})$. The theoretical yield of $\mathrm{HCl}(\mathrm{g})$ was $1.00 \mathrm{mmol}$, which corresponded to $16.0 \mathrm{~mL}$ of gas in the calibrated experimental apparatus.

Comments: Neither method A nor B yielded a measurable amount of gaseous $\mathrm{HCl}$ within 20 min. Thus, the rate of hydrolysis of the substance was negligibly small within this period.

\section{UN 2691 - Phosphorus Pentabromide, PBr5}

Phosphorus pentabromide is a solid with a relative molar mass of 430.49. In method A, $4.00 \mathrm{mmol}$ of water was added to $1.00 \mathrm{mmol}$ of it. In method B, $1.00 \mathrm{mmol}$ of it was added to $20.0 \mathrm{mmol}$ of water. Reaction according to the equation

$$
\mathrm{PBr}_{5}+4 \mathrm{H}_{2} \mathrm{O} \longrightarrow 5 \mathrm{HBr}+\mathrm{OP}(\mathrm{OH})_{3}
$$

was taken as the source of $\mathrm{HBr}$. Generation of $5.00 \mathrm{mmol}$ of gas corresponded to an increase in volume of $80.0 \mathrm{~mL}$ in the calibrated apparatus.

1999 Experimental Results for Phosphorus Pentabromide

\begin{tabular}{|c|c|c|c|c|c|c|c|}
\hline \multicolumn{4}{|c|}{} & \multicolumn{4}{c|}{ Method B } \\
\hline \multirow{2}{*}{$\begin{array}{c}\text { Time } \\
(\mathrm{min})\end{array}$} & $\begin{array}{c}\text { Volume of } \\
\text { Gas }(\mathrm{mL})\end{array}$ & $\begin{array}{c}\text { Mass of } \\
\mathrm{HBr}(\mathrm{g})\end{array}$ & $\begin{array}{c}\text { Yield } \\
(\%)\end{array}$ & $\begin{array}{c}\text { Time } \\
(\mathrm{min})\end{array}$ & $\begin{array}{c}\text { Volume of } \\
\text { Gas }(\mathrm{mL})\end{array}$ & $\begin{array}{c}\text { Mass of } \\
\text { HBr }(\mathrm{g})\end{array}$ & $\begin{array}{c}\text { Yield } \\
(\%)\end{array}$ \\
\hline 1 & 25.9 & 0.131 & 32.4 & 1 & 24.9 & 0.126 & 31.1 \\
\hline 2 & 24.9 & 0.126 & 31.1 & 2 & 3.0 & 0.015 & 3.7 \\
\hline 5 & 24.9 & 0.126 & 31.1 & 5 & 0 & 0 & 0 \\
\hline 10 & 24.9 & 0.126 & 31.1 & 10 & 0 & 0 & 0 \\
\hline
\end{tabular}


2003 Experimental Results for Phosphorus Pentabromide

\begin{tabular}{|c|c|c|c|c|c|c|c|}
\hline \multicolumn{4}{|c|}{ Method A } & \multicolumn{4}{c|}{ Method B } \\
\hline $\begin{array}{c}\text { Time } \\
(\mathrm{min})\end{array}$ & $\begin{array}{c}\text { Volume of } \\
\text { Gas (mL) }\end{array}$ & $\begin{array}{c}\text { Mass of } \\
\mathrm{HBr}(\mathrm{g})\end{array}$ & $\begin{array}{c}\text { Yield } \\
(\%)\end{array}$ & $\begin{array}{c}\text { Time } \\
(\mathrm{min})\end{array}$ & $\begin{array}{c}\text { Volume of } \\
\text { Gas }(\mathrm{mL})\end{array}$ & $\begin{array}{c}\text { Mass of } \\
\mathrm{HBr}(\mathrm{g})\end{array}$ & $\begin{array}{c}\text { Yield } \\
(\%)\end{array}$ \\
\hline 0.5 & 20.5 & 0.104 & 25.6 & 0.5 & 20.1 & 0.102 & 25.1 \\
\hline 1 & 27.2 & 0.138 & 34.4 & 1 & 26.2 & 0.133 & 32.8 \\
\hline 1.5 & 26.5 & 0.134 & 33.1 & 1.5 & 10.5 & 0.053 & 13.1 \\
\hline 2 & 26.2 & 0.133 & 32.8 & 2 & 4.1 & 0.021 & 5.1 \\
\hline 3 & 26.2 & 0.133 & 32.8 & 3 & 0.0 & 0.000 & 0.0 \\
\hline 5 & 26.1 & 0.132 & 32.6 & 5 & 0.0 & 0.000 & 0.0 \\
\hline
\end{tabular}

Comments: In method A, the reaction was rapid and so violent that small droplets of water were spattered onto the walls of the container and out of contact with the $\mathrm{PBr}_{5}$. This initial reaction quickly gave about $35 \%$ of the theoretical amount of $\mathrm{HBr}$ as a gas. This was roughly $2 \mathrm{mmol}$ of $\mathrm{HBr}$ out of the theoretical yield of $5 \mathrm{mmol}$. The initial reaction is therefore better represented as

$$
\mathrm{PBr}_{5}+\mathrm{H}_{2} \mathrm{O} \longrightarrow 2 \mathrm{HBr}+\mathrm{OPBr}_{3}
$$

Subsequent production of $\mathrm{HBr}(\mathrm{g})$ took place only slowly. In method $\mathrm{B}, \mathrm{HBr}(\mathrm{g})$ also formed very rapidly but then dissolved in the excess water within the first $3 \mathrm{~min}$. This indicated that no more than first $2 / 5$ of the theoretical amount of $\operatorname{HBr}(\mathrm{g})$ is unlikely to contribute to a TIH in a real spill. The remaining $3 / 5$ of the $\mathrm{HBr}$ would dissolve as rapidly as it formed.

\section{UN 2692 - Boron Tribromide, BBr3}

Boron tribromide is a colorless liquid with a relative molar mass of 250.54 and a roomtemperature density of $2.650 \mathrm{~g} / \mathrm{cm}^{3}$. In method $\mathrm{A}, 3.00 \mathrm{mmol}$ of water was added to $1.00 \mathrm{mmol}$ of it. In method B, $1.00 \mathrm{mmol}$ of it was mixed with $15.0 \mathrm{mmol}$ of water. Reaction according to the equation

$$
2 \mathrm{BBr}_{3}+2 \mathrm{H}_{2} \mathrm{O} \longrightarrow 3 \mathrm{HBr}+\mathrm{B}(\mathrm{OH})_{3}
$$

was taken as the source of $\operatorname{HBr}(\mathrm{g})$ for computations of mass and yield. The theoretical yield of $\mathrm{HBr}(\mathrm{g})$ was $1.00 \mathrm{mmol}$, which corresponded to $48.0 \mathrm{~mL}$ of gas in the calibrated experimental apparatus. 
2003 Experimental Data for Boron Tribromide

\begin{tabular}{|c|c|c|c|c|c|c|c|}
\hline \multicolumn{4}{|c|}{ Method A } & \multicolumn{4}{c|}{ Method B } \\
\hline $\begin{array}{c}\text { Time } \\
(\mathrm{min})\end{array}$ & $\begin{array}{c}\text { Volume of } \\
\text { Gas (mL) }\end{array}$ & $\begin{array}{c}\text { Mass of } \\
\text { HBr }(\mathrm{g})\end{array}$ & $\begin{array}{c}\text { Yield } \\
(\%)\end{array}$ & $\begin{array}{c}\text { Time } \\
(\mathrm{min})\end{array}$ & $\begin{array}{c}\text { Volume of } \\
\text { Gas }(\mathrm{mL})\end{array}$ & $\begin{array}{c}\text { Mass of } \\
\mathrm{HBr}(\mathrm{g})\end{array}$ & $\begin{array}{c}\text { Yield } \\
(\%)\end{array}$ \\
\hline 0.17 & 35.0 & 0.1770 & 72.9 & 0.17 & 9.0 & 0.0455 & 18.8 \\
\hline 0.35 & 47.6 & 0.2407 & 99.2 & 0.35 & 3.5 & 0.0177 & 7.3 \\
\hline 0.5 & 48.9 & 0.2473 & 102 & 0.5 & 1.5 & 0.0076 & 3.1 \\
\hline 1 & 49.8 & 0.2519 & 104 & 1 & 0.6 & 0.0030 & 1.3 \\
\hline 2 & 49.9 & 0.2524 & 104 & 2 & 0.2 & 0.0010 & 0.4 \\
\hline 5 & 49.9 & 0.2524 & 104 & 5 & 0.0 & 0.0000 & 0.0 \\
\hline
\end{tabular}

Comments: In method $\mathrm{A}$, the reaction was explosively rapid and generated the full theoretical amount of $\operatorname{HBr}(\mathrm{g})$ within $5 \mathrm{~s}$. In method $\mathrm{B}$, the reaction was again very rapid. Essentially all of the $\operatorname{HBr}(\mathrm{g})$ that formed quickly dissolved in the excess water to produce $\mathrm{HBr}(\mathrm{aq})$ (note the quick maximum on the yield curve).

\section{UN 2806 - Lithium Nitride, $\mathrm{Li}_{3} \mathrm{~N}$}

Lithium nitride is a solid with a relative molar mass of 34.82. In method A, $1.12 \mathrm{mmol}$ of water was added to $0.373 \mathrm{mmol}$ of it. In method B, $0.373 \mathrm{mmol}$ of it was mixed with $5.60 \mathrm{mmol}$ of water. Reaction according to the equation

$$
\mathrm{Li}_{3} \mathrm{~N}+3 \mathrm{H}_{2} \mathrm{O} \longrightarrow 3 \mathrm{LiOH}+\mathrm{NH}_{3}
$$

was taken as the source of $\mathrm{NH}_{3}(\mathrm{~g})$ for computations of mass and yield. The theoretical yield of $\mathrm{NH}_{3}(\mathrm{~g})$ was $0.373 \mathrm{mmol}$, which corresponded to $5.96 \mathrm{~mL}$ of gas in the calibrated experimental apparatus.

2003 Experimental Data for Lithium Nitride

\begin{tabular}{|c|c|c|c|c|c|c|c|}
\hline \multicolumn{4}{|c|}{ Method A } & \multicolumn{4}{c|}{ Method B } \\
\hline $\begin{array}{c}\text { Time } \\
(\mathrm{min})\end{array}$ & $\begin{array}{c}\text { Volume of } \\
\text { Gas (mL) }\end{array}$ & $\begin{array}{c}\text { Mass of } \\
\mathrm{NH}_{3}(\mathrm{~g})\end{array}$ & $\begin{array}{c}\text { Yield } \\
(\%)\end{array}$ & $\begin{array}{c}\text { Time } \\
(\mathrm{min})\end{array}$ & $\begin{array}{c}\text { Volume of } \\
\text { Gas }(\mathrm{mL})\end{array}$ & $\begin{array}{c}\text { Mass of } \\
\mathrm{NH}_{3}(\mathrm{~g})\end{array}$ & $\begin{array}{c}\text { Yield } \\
(\%)\end{array}$ \\
\hline 0.167 & 6.0 & 0.00619 & 97.4 & 0.167 & 6.0 & 0.00619 & 97.4 \\
\hline 0.5 & 6.0 & 0.00619 & 97.4 & 0.5 & 6.0 & 0.00619 & 97.4 \\
\hline 1 & 6.0 & 0.00619 & 97.4 & 1 & 5.9 & 0.00609 & 95.8 \\
\hline 2 & 6.0 & 0.00619 & 97.4 & 2 & 5.8 & 0.00599 & 94.2 \\
\hline 5 & 6.0 & 0.00619 & 97.4 & 5 & 5.7 & 0.00588 & 92.5 \\
\hline & & & & 10 & 5.70 & 0.00588 & 92.5 \\
\hline
\end{tabular}


Comments: Less than $1.00 \mathrm{mmol}$ of substance was used because of the violence of the reaction. The safe amount was established in preliminary tests. A flame-like flash occurred instantly upon mixing the $\mathrm{Li}_{3} \mathrm{~N}$ and water. This suggested that some air-oxidation [according to the equation: $2 \mathrm{Li}_{3} \mathrm{~N}+\mathrm{O}_{2} \longrightarrow 3 \mathrm{Li}_{2} \mathrm{O}+2 \mathrm{NO}_{2}(\mathrm{~g})$, for example] might have occurred. However, the product gases were colorless, and $\mathrm{NO}_{2}(\mathrm{~g})$ is brown. Close to the theoretical amount of $\mathrm{NH}_{3}(\mathrm{~g})$ was generated very rapidly in both methods. When excess water was present (method B), some of the $\mathrm{NH}_{3}(\mathrm{~g})$ dissolved to give $\mathrm{NH}_{3}(\mathrm{aq})$. However, the excess water in method $\mathrm{B}$ had a high concentration of dissolved by-product LiOH, which greatly limited the dissolution of the $\mathrm{NH}_{3}(\mathrm{~g})$.

\section{UN2806 (Surrogate) — Magnesium Nitride, $\operatorname{Mg}_{3} \mathrm{~N}_{2}$}

Magnesium nitride was studied as a surrogate for lithium nitride (UN 2806). Magnesium nitride is a solid with a relative molar mass of 100.95. In method A, $6.00 \mathrm{mmol}$ of water was added to $1.00 \mathrm{mmol}$ of it. In method B, $1.00 \mathrm{mmol}$ of it was mixed with $30.0 \mathrm{mmol}$ of water. Reaction according to the equation

$$
\mathrm{Mg}_{3} \mathrm{~N}_{2}+6 \mathrm{H}_{2} \mathrm{O} \longrightarrow 2 \mathrm{NH}_{3}+3 \mathrm{Mg}(\mathrm{OH})_{2}
$$

was taken as the source of $\mathrm{NH}_{3}(\mathrm{~g})$ for computations of mass and yield. The theoretical yield of $\mathrm{NH}_{3}$ (g) was $2.00 \mathrm{mmol}$, which corresponded to $32.0 \mathrm{~mL}$ of gas in the calibrated experimental apparatus.

\section{Experimental Data for Magnesium Nitride}

\begin{tabular}{|c|c|c|c|c|c|c|c|}
\hline \multicolumn{4}{|c|}{ Method A } & \multicolumn{4}{c|}{ Method B } \\
\hline $\begin{array}{c}\text { Time } \\
(\mathrm{min})\end{array}$ & $\begin{array}{c}\text { Volume of } \\
\text { Gas }(\mathrm{mL})\end{array}$ & $\begin{array}{c}\text { Mass of } \\
\mathrm{NH}_{3}(\mathrm{~g})\end{array}$ & $\begin{array}{c}\text { Yield } \\
(\%)\end{array}$ & $\begin{array}{c}\text { Time } \\
(\mathrm{min})\end{array}$ & $\begin{array}{c}\text { Volume of } \\
\text { Gas }(\mathrm{mL})\end{array}$ & $\begin{array}{c}\text { Mass of } \\
\mathrm{NH}_{3}(\mathrm{~g})\end{array}$ & $\begin{array}{c}\text { Yield } \\
(\%)\end{array}$ \\
\hline 0.33 & 16.0 & 0.0168 & 49.2 & 0.33 & 22.0 & 0.0231 & 67.7 \\
\hline 0.67 & 16.3 & 0.0171 & 50.2 & 0.5 & 26.4 & 0.0277 & 81.2 \\
\hline 1 & 17.0 & 0.0178 & 52.3 & 1 & 20.6 & 0.0216 & 63.4 \\
\hline 2 & 17.8 & 0.0187 & 54.8 & 2 & 15.8 & 0.0166 & 48.6 \\
\hline 3 & 18.2 & 0.0191 & 56.0 & 3 & 13.0 & 0.0136 & 40.0 \\
\hline 5 & 18.4 & 0.0193 & 56.6 & 5 & 10.0 & 0.0105 & 30.8 \\
\hline 10 & 18.6 & 0.0195 & 57.2 & 7 & 8.4 & 0.0088 & 25.8 \\
\hline 20 & 18.8 & 0.0197 & 57.8 & 10 & 7.0 & 0.0073 & 21.5 \\
\hline & & & & 15 & 5.8 & 0.0061 & 17.8 \\
\hline & & & & 20 & 5.2 & 0.0054 & 16.0 \\
\hline & & & & 30 & 5.0 & 0.0052 & 15.4 \\
\hline
\end{tabular}

Comments: This substance, which does not have a UN number, was tested because it is chemically related to lithium nitride, which reacted explosively with water. The reaction in this case was less violent, although still quite rapid. In method $\mathrm{A}$, the yield of $\mathrm{NH}_{3}(\mathrm{~g})$ was only about 
half of the theoretical yield. In method $\mathrm{B}$, the maximum on the yield curve indicated that $\mathrm{NH}_{3}(\mathrm{~g})$ that formed at the start of the reaction later dissolved to form $\mathrm{NH}_{3}(\mathrm{aq})$. In this case, the byproduct of the hydrolysis reaction, $\operatorname{Mg}(\mathrm{OH})_{2}$, is only sparingly soluble in water. The excess water was accordingly not as basic as it would have been if a soluble base had formed and more $\mathrm{NH}_{3}(\mathrm{~g})$ had dissolved.

\section{UN 3246 - Methanesulfonyl Chloride, $\mathrm{CH}_{3} \mathrm{SO}_{2} \mathrm{Cl}$}

Methanesulfonyl chloride is a yellow liquid with a relative molar mass of 114.54 and a room-temperature density of $1.485 \mathrm{~g} / \mathrm{cm}^{3}$. In method A, $1.00 \mathrm{mmol}$ of it was mixed with 1.00 mmol of water. In method B, $1.00 \mathrm{mmol}$ was mixed with $5.00 \mathrm{mmol}$ of water. The reaction

$$
\mathrm{CH}_{3} \mathrm{SO}_{2} \mathrm{Cl}+\mathrm{H}_{2} \mathrm{O} \longrightarrow \mathrm{HCl}+\mathrm{CH}_{3} \mathrm{SO}_{2} \mathrm{OH}
$$

was proposed as the source of $\mathrm{HCl}(\mathrm{g})$. The theoretical yield of $\mathrm{HCl}(\mathrm{g})$ was $1.00 \mathrm{mmol}$, which corresponded to $16.0 \mathrm{~mL}$ of gas in the calibrated experimental apparatus.

Comments: Neither method A nor B generated any measurable gases within 20 min. 
APPENDIX D:

ADDITIONAL DETAILS ON WATER-REACTIVE MATERIALS 


\section{APPENDIX D:}

\section{ADDITIONAL DETAILS ON WATER-REACTIVE MATERIALS}

This appendix provides detailed information on the water-reactive materials listed in the Table of Initial Isolation and Protective Action Distances. We begin in Section D.1 with a detailed discussion of how we reduce the experimental data listed in Appendix $\mathrm{C}$ to determine the key parameters necessary to model TIHWR releases. The parameters themselves for all TIHWR materials considered in the ERG2004 are then provided in Section D.2. Finally, Section D.3 explains why various materials were selected for the TIHWR list and reviews changes from previous recommendations.

\section{D.1 ANALYSIS OF EXPERIMENTAL DATA: EXTRACTION OF KEY PARAMETERS}

As discussed in Section 3.3, we assume that the evolution of a TIHWR gas can be modeled (for most materials) using the first-order rate equation

$$
M(t)=M_{s t} \beta\left(1-e^{-\lambda t}\right)
$$

where $M_{\mathrm{st}}$ is the stoichiometric mass that could evolve, and $M(t)$ is the total mass of TIH gas actually emitted by time $t$ from the spill. The parameter $\lambda$ is the first-order rate constant for the process, and $\beta$ is an empirically determined efficiency factor defined from Eq. D.1 as

$$
\beta=\frac{M_{\infty}}{M_{s t}}
$$

where $M_{\infty}$ is the maximum mass of TIH gas that is evolved from the water at long times. Note that more product may be formed than actually evolves from the water due to dissolution of the gas in the water, as discussed in Section D.1.3. We generally determine these parameters by least-squares fitting from the amount of TIH gas emitted as a function of time, as observed in our method B experiments.

Four types of behavior were observed in the 55 experiments performed in 1999 and 2003 (for the 2000 and 2004 ERGs, respectively). They are described in the following subsections.

\section{D.1.1 No Emissions}

Some experiments produced no TIH gases in either method (A or B), even though we had descriptive evidence that they were quite reactive with water. These numbered 13 , or $24 \%$ of cases measured. An additional two materials (phosphorus tribromide and phosphorus oxybromide) exhibited slow emissions in method A but no emissions with method B due to 
sequestration of the evolved gas by the excess water. Null results for these chemicals illustrate the importance of actual experiments over anecdotal information from the chemistry literature.

\section{D.1.2 Simple First-Order Process}

Twelve experiments (22\%) showed the simple first-order process described above. A typical example of these simple cases is shown in Figure D.1. As shown, we used the actual observed maximum mass of gas evolved, $M_{\infty}$, divided by the stoichiometric maximum $M_{\mathrm{st}}$ to determine $\beta$. Using a least-squares fit for $M_{\infty}$ gave almost identical results in all cases.

\section{D.1.3 First-Order Process with Dissolution}

Many experiments showed an apparent first-order process where the evolved TIH gas dissolved back into the water, thus serving as a removal mechanism. Twenty-three out of the 55 experiments, or approximately $45 \%$, showed this behavior, an example of which is shown in Figure D.2. Because the TIH gas was kept in a closed environment in contact with water in the experimental apparatus, dissolution was promoted. In the natural environment, the TIH gas produced would likely bubble out of the water quickly and dilute in the atmosphere, thus avoiding significant dissolution. For this reason, $M_{\infty}$ was determined with a least-squares fit of the data to obtain the completion fraction $\beta$, rather than simply using the observed maximum that occurred as dissolution overcame the rate of evolution. This procedure may tend to overestimate the source term for atmospheric dispersion of the TIH gas. Three subcases of strong dissolution materials were identified and treated differently:

1. Three or more rising points. For 7 of the 23 dissolution cases, the data contained 3 or more points, as shown in Figure D.2. In these cases, the first-order rate curve resulting from fitting only the rising points is overdetermined and the goodness of fit confirms the appropriateness of a first-order model.

2. Two rising points. For 8 of the 23 dissolution cases, only two points were obtained on the rising portion of the $M(\mathrm{t})$ curve due to the rapidity with which dissolution occurred. The origin and these two points exactly determine the parameters $M_{\infty}$ and $\lambda$, and the fit to the model is perfect. These cases were handled in the same way as those with 3 or more meaningful measurements. An example of such a case is given in Figure D.3.

3. Only one rising point. Finally, in Figure D. 4 we show an example of the other 8 dissolution cases, for which only the first nontrivial measurement was higher and the second and subsequent evolved mass values had decreased. These represent cases in which dissolution of the gas competes strongly with production. These cases generally exhibited very fast reaction rates as well. For these chemicals, it is not possible to determine two parameters from a fit. Therefore, we determined the rate constant $\lambda$ by fitting a first-order process to the data obtained in method $\mathrm{A}$, in which 


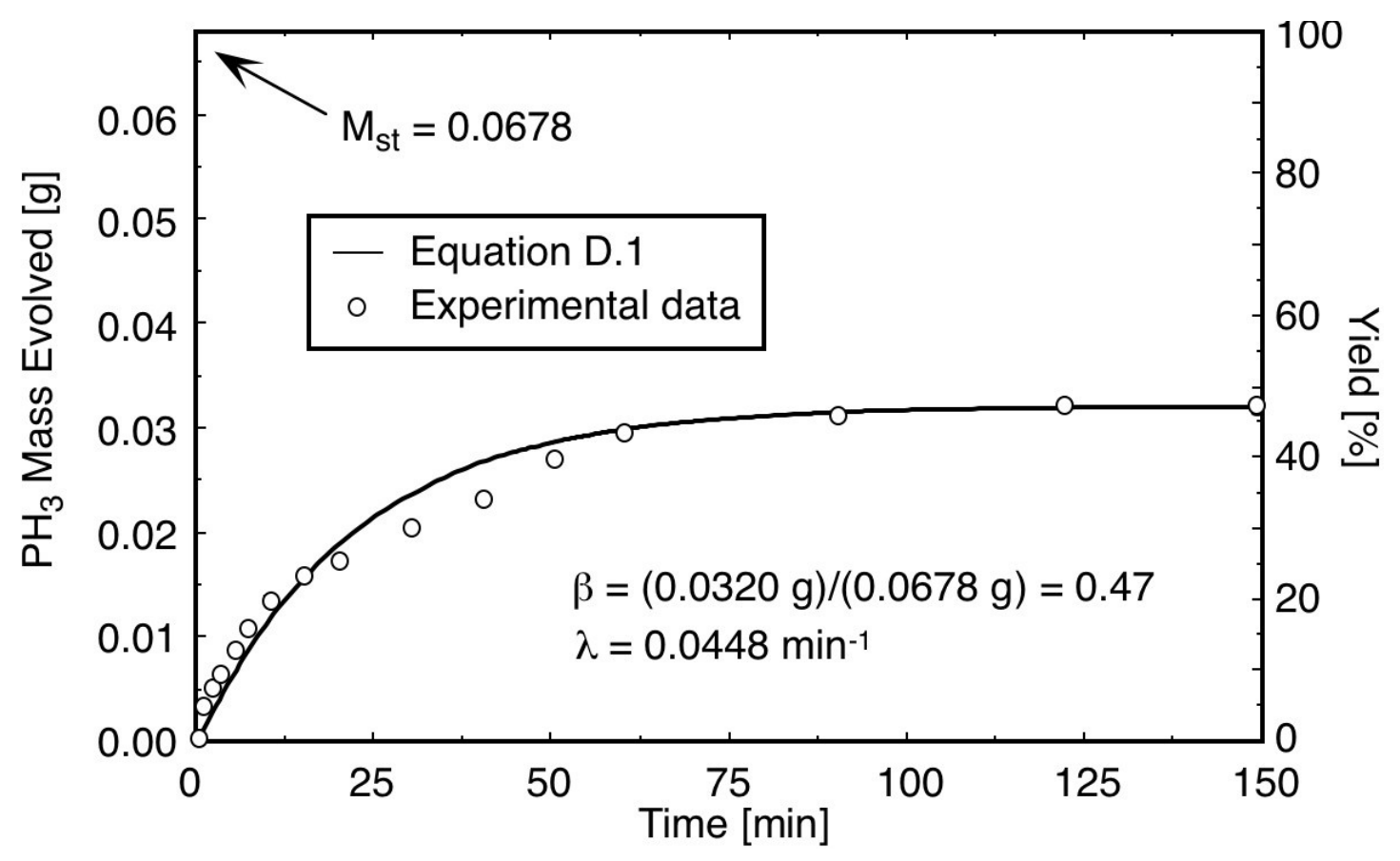

FIGURE D.1 Example of a Simple First-Order Process from the Experimental Series Together with the Derived Parameters for Eq. D.1. Results are shown for calcium phosphide (UN 1360).

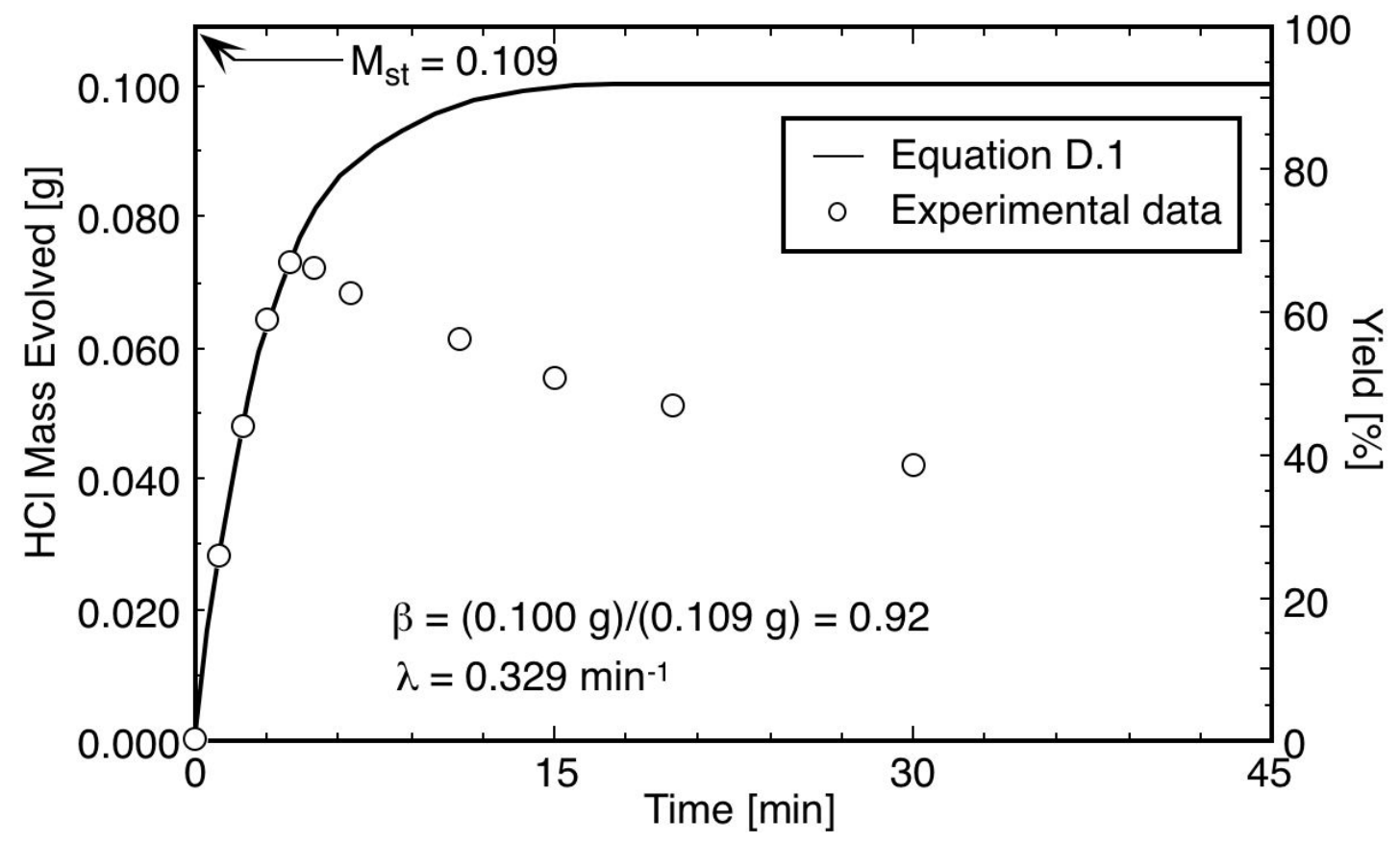

FIGURE D.2 Example of a First-Order Process from the Experimental Series where Dissolution of the TIH Product Competes with the Evolution. Data are shown together with the derived parameters for Eq. D.1. Results are shown for ethyltrichlorosilane (UN 1196). 


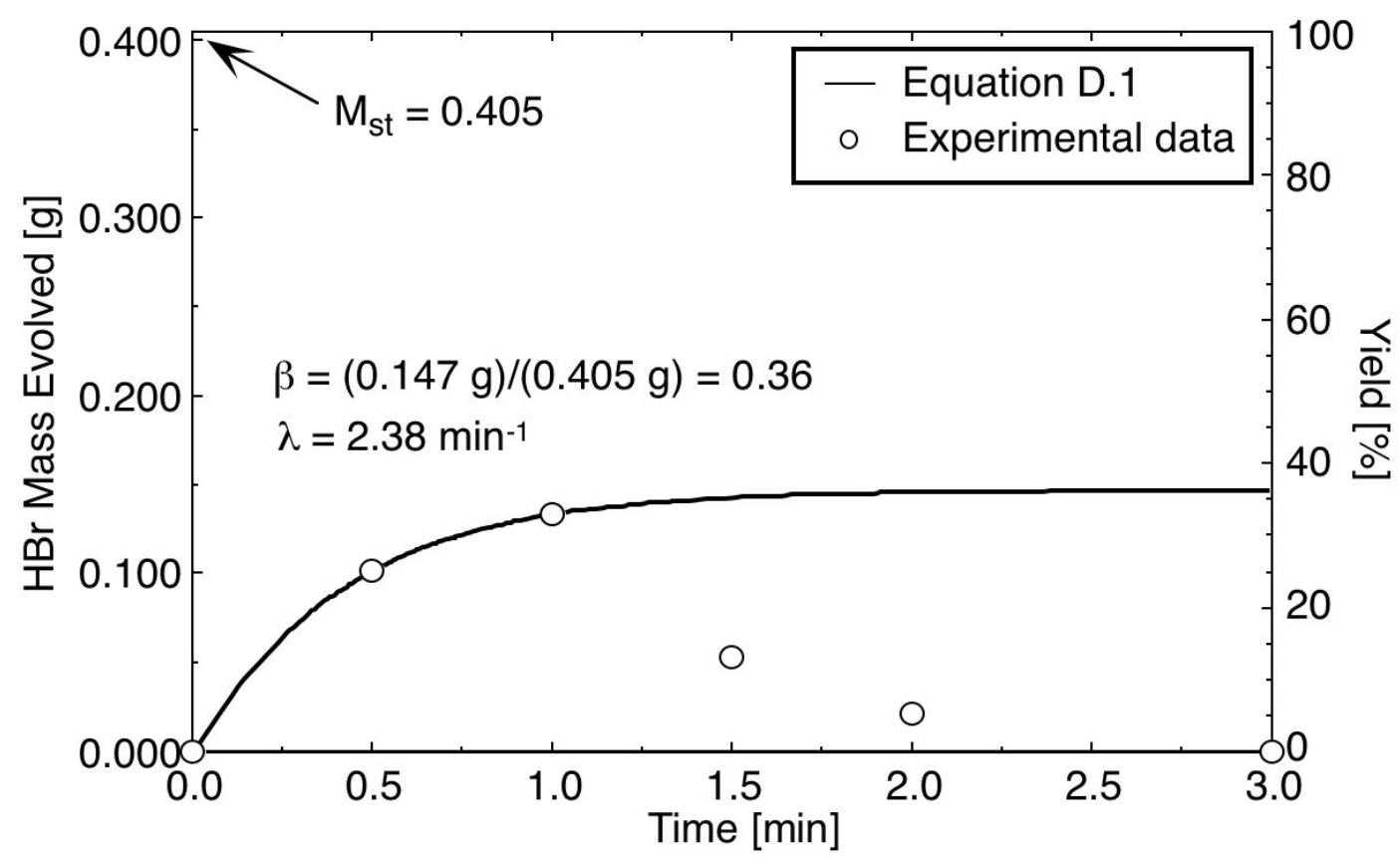

FIGURE D.3 Example of a First-Order Rate Process Competing with Dissolution of the TIH Gas for which only Two Rising Points were Measured. Data are shown together with the derived parameters for Eq. D.1. Results are shown for phosphorus pentabromide (UN 2691).

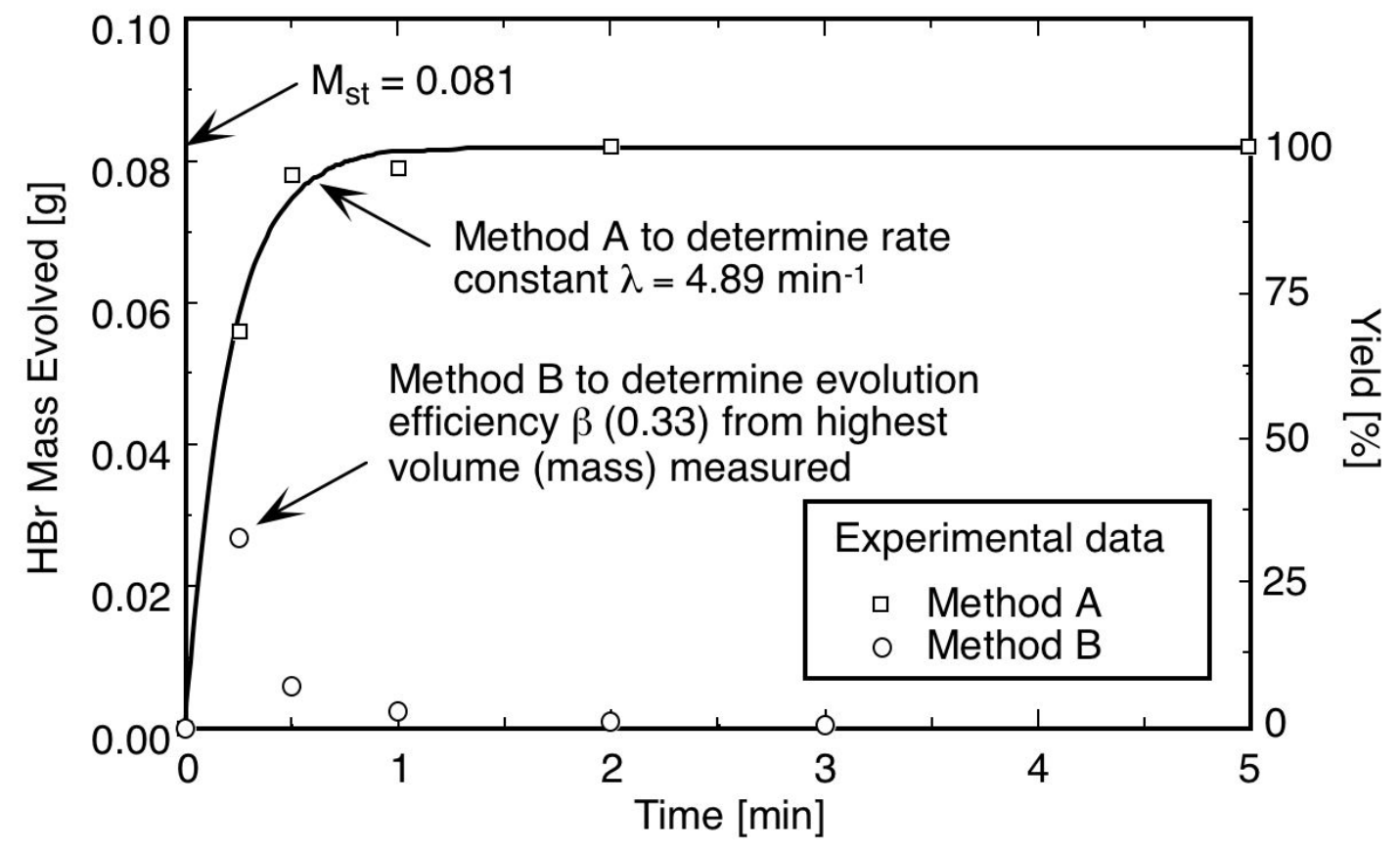

FIGURE D.4 Example of a First-Order Rate Process Competing with Dissolution of the TIH Gas for which only One Rising Point was Measured. Data are shown together with the derived parameters for Eq. D.1. Results are shown for acetyl bromide (UN 1716). 
water was not in stoichiometric excess. Then, rather than using the known $\lambda$ to fit the one nontrivial point to a first-order process and find $M_{\infty}$, we used the largest evolved TIH gas mass actually observed, $M_{\max }$, to obtain $\beta$ as the ratio $M_{\max } / M_{\text {st }}$. This procedure reflects the very rapid dissolution that occurs in these cases. To extrapolate an exponential fitted in this way to find $M_{\infty}$ is probably too conservative.

Two exceptional cases were observed: nitrosylsulfuric acid (UN 2308) and lithium nitride (UN 2806). The nitrosylsulfuric acid experiment was performed in 1999 but not repeated in 2003. In this case, the method A experiment also gave a single high initial point followed by a rapid decrease of the gas volume to zero. Therefore, we could not fit the method A data to find $\lambda$, but had to estimate it based on the maximum observed gas volume, which occurred at $t=1 \mathrm{~min}$. The lithium nitride experiment was performed in 2003, and produced $97 \%$ of the stoichiometric maximum amount of $\mathrm{NO}_{2}$ after $10 \mathrm{~s}$, in both methods $\mathrm{A}$ and $\mathrm{B}$. Again, we could not use method A data to fit for a value of $\lambda$. We estimated it as $6 \mathrm{~min}^{-1}$, the inverse of the smallest time studied.

\section{D.1.4 Autocatalytic Reactions}

In 5 of the 55 experiments, we observed a more complex pattern of reaction. These cases all involved silanes but represented only $23 \%$ of the 22 silanes studied. In these cases, a polymeric by-product formed during the first stages of the reaction apparently slowed the reaction of the remaining silane with water. A slow emission of gas at a constant rate ensued for a period of 2-10 $\mathrm{min}$. At that point the reactions appeared to autocatalyze, and subsequently followed what appeared to be a normal first-order reaction process. In these cases, we used a four-parameter fit. The values of $M_{\infty}$ and $\lambda$ were given their normal meaning, but the time was measured from $T_{\text {ind }}$, a new parameter that indicated when the autocatalysis began strongly. The mass of gas that had evolved during the interval from $t=0$ to $t=T_{\text {ind }}$ was also used as a parameter, called $m_{i}$. The formal relationships are as follows:

$$
\begin{array}{cc}
M(t)=m_{i} \frac{t}{T_{\text {ind }}} & \left(t \leq T_{\text {ind }}\right) \\
M(t)=m_{i}+M_{\text {st }} \beta^{\prime}\left(1-e^{-\lambda\left(t-T_{\text {ind }}\right)}\right), & \left(t>T_{\text {ind }}\right)
\end{array}
$$

where

$$
\beta^{\prime}=\beta-\frac{M_{i}}{M_{s t}} .
$$

These formulas are continuous at $t=T_{\text {ind }}$, but their slopes are not. Nevertheless, they give a good fit to the time dependence of the data in these cases. Figure D.5 illustrates this behavior and the goodness of the fit. 


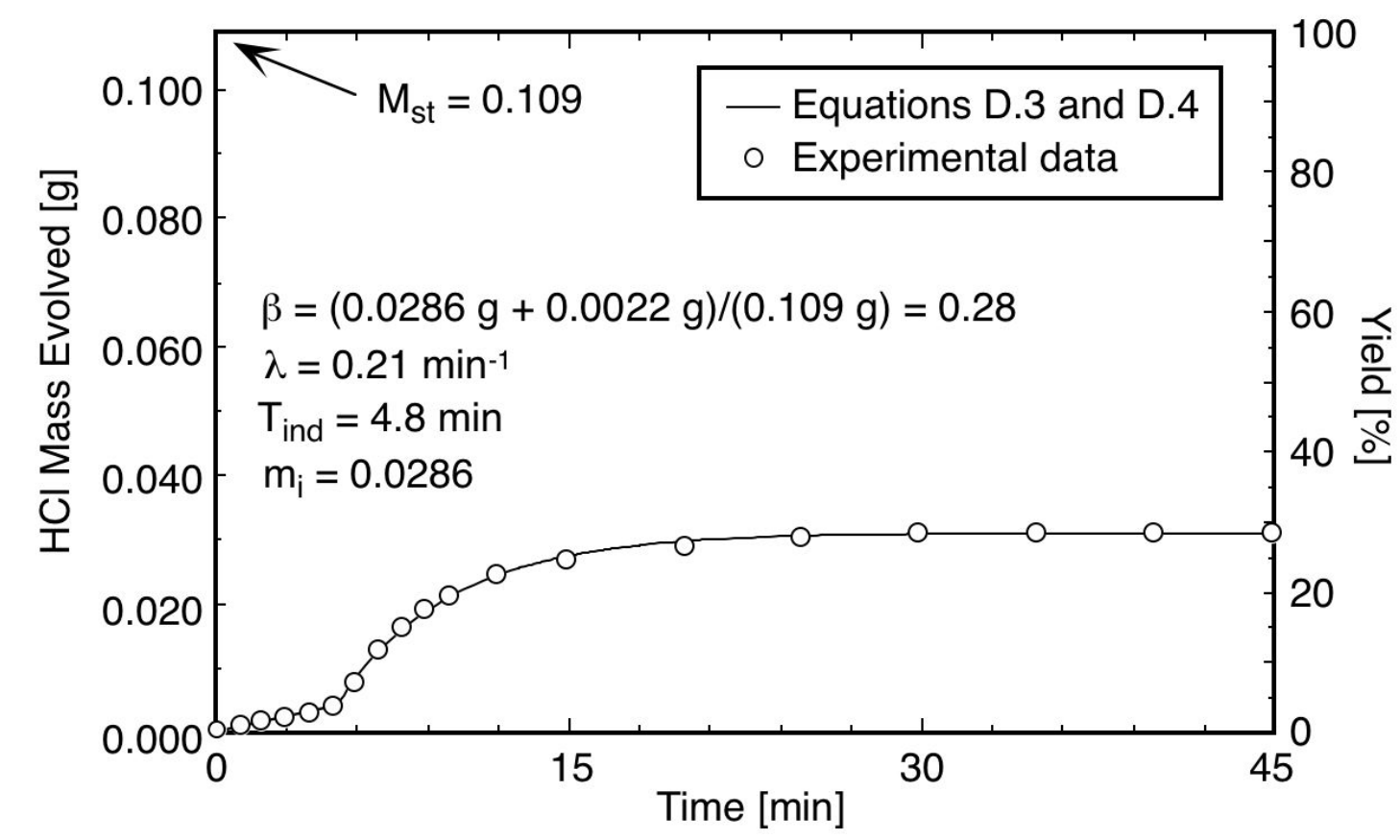

FIGURE D.5 Example of an Autocatalytic Reaction with an Initial By-Product Greatly Reducing Availability of Water for Subsequent Reaction. Data are shown together with the derived parameters for Eqs. D.3 and D.4. Results are shown for cyclohexyltrichlorosilane (UN 1763).

\section{D.1.5 Summary}

The four types of emission time dependence, along with the three in which dissolution and production competed significantly, describe all of the 55 cases studied experimentally. The parameters obtained by fitting the data were used in CASRAM to describe the time dependent emissions of TIH gas when a water-reactive substance is spilled into water. Larger-scale experiments in the future should verify that these millimole results do indeed give a good approximation of an actual large-scale spill.

\section{D.2 KEY PARAMETERS EMPLOYED IN THE TIWHR ANALYSIS}

Supplementary information on all water-reactive materials in the TIHWR list is provided in Tables D.1 and D.2. 
TABLE D.1 Supplementary Information on Water-Reactive Materials in the ERG2004 for Materials Modeled by a Simple First-order Process ${ }^{a}$

\begin{tabular}{|c|c|c|c|c|c|c|c|c|c|}
\hline $\begin{array}{l}\text { UN } \\
\text { No. }\end{array}$ & Name & ERG & Type & Prod. & St & Dens. & S.Y. & $\beta$ & $\begin{array}{c}\lambda_{\mathrm{o}} \\
\left(\mathrm{min}^{-1}\right)\end{array}$ \\
\hline 1162 & Dimethyldichlorosilane & $00 / 04$ & 2 & $\mathrm{HCl}$ & $\mathrm{L}$ & 1.10 & 0.565 & 0.41 & 0.935 \\
\hline 1196 & Ethyltrichlorosilane & 04 & 3.1 & $\mathrm{HCl}$ & $\mathrm{L}$ & 1.238 & 0.669 & 0.92 & 0.329 \\
\hline 1242 & Methyldichlorosilane & 04 & 3.2 & $\mathrm{HCl}$ & $\mathrm{L}$ & 1.11 & 0.634 & 0.60 & 9.24 \\
\hline 1250 & Methyltrichlorosilane & $00 / 04$ & 2 & $\mathrm{HCl}$ & $\mathrm{L}$ & 1.27 & 0.732 & 0.33 & 1.31 \\
\hline 1295 & Trichlorosilane & & & $\mathrm{HCl}$ & $\mathrm{L}$ & 1.34 & 0.808 & 0.41 & 1.14 \\
\hline 1298 & Trimethylchlorosilane & $00 / 04$ & 2 & $\mathrm{HCl}$ & $\mathrm{L}$ & 0.85 & 0.336 & 0.37 & 1.45 \\
\hline 1305 & Vinyltrichlorosilane & 04 & 3.2 & $\mathrm{HCl}$ & $\mathrm{L}$ & 1.26 & 0.681 & 0.60 & 1.53 \\
\hline 1340 & Phosphorus pentasulfide & & & $\mathrm{H}_{2} \mathrm{~S}$ & $\mathrm{~S}$ & 2.09 & 0.766 & 0.6 & 0.2 \\
\hline 1360 & Calcium phosphide & $00 / 04$ & 2 & $\mathrm{PH}_{3}$ & $\mathrm{~S}$ & 2.51 & 0.373 & 0.47 & 0.045 \\
\hline 1384 & Sodium hydrosulfite & 00 & 1 & $\mathrm{H}_{2} \mathrm{~S}$ & S & 0.9 & 0.390 & 0.02 & 5 \\
\hline 1397 & Aluminum phosphide & & & $\mathrm{PH}_{3}$ & $\mathrm{~S}$ & 2.40 & 0.587 & 0.47 & 0.045 \\
\hline 1412 & Lithium amide & 04 & 3.2 & $\mathrm{NH}_{3}$ & $\mathrm{~S}$ & 1.18 & 0.740 & 0.24 & 4.51 \\
\hline 1419 & Magnesium aluminum phosphide & & & $\mathrm{PH}_{3}$ & S & 2.20 & 0.529 & 0.47 & 0.05 \\
\hline 1432 & Sodium phosphide & & & $\mathrm{PH}_{3}$ & S & 1.74 & 0.283 & 0.47 & 0.05 \\
\hline 1541 & Acetone cyanohydrin & 04 & 1 & $\mathrm{HCN}$ & $\mathrm{L}$ & 0.93 & 0.317 & 0.3 & 0.1 \\
\hline 1680 & Potassium cyanide & & & $\mathrm{HCN}$ & $\mathrm{S}$ & 1.52 & 0.415 & 0.4 & 0.1 \\
\hline 1689 & Sodium cyanide & & & $\mathrm{HCN}$ & $\mathrm{S}$ & 1.52 & 0.552 & 0.4 & 0.1 \\
\hline 1716 & Acetyl bromide & 04 & 3.3 & $\mathrm{HBr}$ & $\mathrm{L}$ & 1.66 & 0.658 & 0.33 & 4.89 \\
\hline 1717 & Acetyl chloride & 04 & 3.3 & $\mathrm{HCl}$ & $\mathrm{L}$ & 1.11 & 0.464 & 0.38 & 3.15 \\
\hline 1724 & Allyl trichlorosilane, stabilized & 04 & 3.2 & $\mathrm{HCl}$ & $\mathrm{L}$ & 1.21 & 0.623 & 0.76 & 1.06 \\
\hline 1725 & Aluminum bromide, anhydrous & 00 & 1 & $\mathrm{HBr}$ & $\mathrm{S}$ & 2.54 & 0.910 & 0.5 & 0.1 \\
\hline 1726 & Aluminum chloride, anhydrous & 00 & 2 & $\mathrm{HCl}$ & $\mathrm{S}$ & 2.44 & 0.820 & 0.5 & 0.2 \\
\hline 1732 & Antimony pentafluoride & 04 & 1 & $\mathrm{HF}$ & $\mathrm{L}$ & 2.99 & 0.461 & 0.4 & 5 \\
\hline 1745 & Bromine pentafluoride $\mathrm{b}^{\mathrm{b}}$ & & & $\mathrm{Br}_{2}$ & $\mathrm{~L}$ & 2.47 & 0.456 & 0.04 & 0.2 \\
\hline 1745 & Bromine pentafluoride $\mathrm{b}$ & & & $\mathrm{HF}$ & $\mathrm{L}$ & 2.47 & 0.572 & 0.4 & 5 \\
\hline 1746 & Bromine trifluoride $^{\mathrm{b}}$ & & & $\mathrm{Br}_{2}$ & $\mathrm{~L}$ & 2.80 & 0.591 & 0.02 & 0.2 \\
\hline 1746 & Bromine trifluoride ${ }^{\mathrm{b}}$ & & & $\mathrm{HF}$ & $\mathrm{L}$ & 2.80 & 0.438 & 0.4 & 5 \\
\hline 1752 & Chloroacetyl chloride & $00 / 04$ & 3.1 & $\mathrm{HCl}$ & $\mathrm{L}$ & 1.50 & 0.323 & 0.1 & 0.391 \\
\hline 1754 & Chlorosulfonic acid & 04 & 3.3 & $\mathrm{HCl}$ & $\mathrm{L}$ & 1.76 & 0.313 & 0.91 & 7.31 \\
\hline 1758 & Chromium oxychloride & 04 & 2 & $\mathrm{HCl}$ & $\mathrm{L}$ & 1.91 & 0.471 & 0.09 & 0.067 \\
\hline 1766 & Dichlorophenyltrichlorosilane $\mathrm{c}^{\mathrm{c}}$ & 04 & 3.2 & $\mathrm{HCl}$ & $\mathrm{L}$ & 1.56 & 0.84 & 0.61 & 1.96 \\
\hline 1767 & Diethyldichlorosilane & 04 & 2 & $\mathrm{HCl}$ & $\mathrm{L}$ & 1.05 & 0.464 & 0.21 & 0.0364 \\
\hline 1769 & Diphenyldichlorosilane & 04 & 2 & $\mathrm{HCl}$ & $\mathrm{L}$ & 1.22 & 0.288 & 0.12 & 0.129 \\
\hline 1771 & Dodecyltrichlorosilane & 04 & 2 & $\mathrm{HCl}$ & $\mathrm{L}$ & 1.03 & 0.359 & 0.33 & 0.0733 \\
\hline 1777 & Fluorosulfonic acid & & & $\mathrm{HF}$ & $\mathrm{L}$ & 1.73 & 0.200 & 0.1 & 5 \\
\hline 1799 & Nonyltrichlorosilane & 04 & 2 & $\mathrm{HCl}$ & $\mathrm{L}$ & 1.0 & 0.417 & 0.55 & 0.037 \\
\hline 1801 & Octyltrichlorosilane & 04 & 2 & $\mathrm{HCl}$ & $\mathrm{L}$ & 1.33 & 0.442 & 0.3 & 0.109 \\
\hline 1804 & Phenyltrichlorosilane & 04 & 3.1 & $\mathrm{HCl}$ & $\mathrm{L}$ & 1.321 & 1.035 & 0.68 & 0.399 \\
\hline 1806 & Phosphorus pentachloride & 04 & 3.3 & $\mathrm{HCl}$ & $\mathrm{S}$ & 1.60 & 0.875 & 0.22 & 6.13 \\
\hline 1809 & Phosphorus trichloride & 00 & 3.2 & $\mathrm{HCl}$ & $\mathrm{L}$ & 1.57 & 0.796 & 0.43 & 1.72 \\
\hline 1810 & Phosphorus oxychloride & $00 / 04$ & 3.3 & $\mathrm{HCl}$ & $\mathrm{L}$ & 1.67 & 0.713 & 0.85 & 1.90 \\
\hline 1816 & Propyltrichlorosilane & $00 / 04$ & 3.1 & $\mathrm{HCl}$ & $\mathrm{L}$ & 1.20 & 0.618 & 0.45 & 0.45 \\
\hline 1818 & Silicon tetrachloride & 04 & 3.1 & $\mathrm{HCl}$ & $\mathrm{L}$ & 1.48 & 0.858 & 0.37 & 2.20 \\
\hline 1828 & Sulfur chlorides ${ }^{\mathrm{b}}$ & & & $\mathrm{SO}_{2}$ & $\mathrm{~L}$ & 1.62 & 0.252 & 0.12 & 0.2 \\
\hline 1828 & Sulfur chlorides ${ }^{b}$ & & & $\mathrm{HCl}$ & $\mathrm{L}$ & 1.62 & 0.540 & 0.4 & 5 \\
\hline 1828 & Sulfur chlorides ${ }^{\mathrm{b}}$ & & & $\mathrm{H}_{2} \mathrm{~S}$ & $\mathrm{~L}$ & 1.62 & 0.474 & 0.1 & 0.6 \\
\hline 1834 & Sulfuryl chloride ${ }^{b}$ & 00 & 3.1 & $\mathrm{HCl}$ & $\mathrm{L}$ & 1.66 & 0.540 & 0.2 & 0.92 \\
\hline 1836 & Thionyl chloride $\mathrm{b}^{\mathrm{b}}$ & 04 & 3.1 & $\mathrm{SO}_{2}$ & $\mathrm{~L}$ & 1.63 & 0.538 & 1 & 2.75 \\
\hline 1836 & Thionyl chloride $\mathrm{b}$ & 04 & 3.1 & $\mathrm{HCl}$ & $\mathrm{L}$ & 1.63 & 0.613 & 0.59 & 3.02 \\
\hline 1838 & Titanium tetrachloride & 00 & 3.1 & $\mathrm{HCl}$ & $\mathrm{L}$ & 1.73 & 0.769 & 0.24 & 1.35 \\
\hline
\end{tabular}


TABLE D.1 (Cont.)

\begin{tabular}{|c|c|c|c|c|c|c|c|c|c|}
\hline $\begin{array}{l}\text { UN } \\
\text { No. }\end{array}$ & Name & ERG & Type & Prod. & St & Dens. & S.Y. & $\beta$ & $\begin{array}{c}\lambda_{\mathrm{o}} \\
\left(\mathrm{min}^{-1}\right)\end{array}$ \\
\hline 1898 & Acetyl iodide & & & HI & $\mathrm{L}$ & 2.07 & 0.753 & 0.4 & 0.2 \\
\hline 1923 & Calcium hydrosulfite & & & $\mathrm{H}_{2} \mathrm{~S}$ & $\mathrm{~S}$ & 1.0 & 0.327 & 0.02 & 5 \\
\hline 1931 & Zinc hydrosulfite & & & $\mathrm{H}_{2} \mathrm{~S}$ & $\mathrm{~S}$ & 0.7 & 0.384 & 0.02 & 5 \\
\hline 2004 & Magnesium diamide & & & $\mathrm{NH}_{3}$ & $\mathrm{~S}$ & 1.39 & 0.604 & 0.97 & 6 \\
\hline 2011 & Magnesium phosphide & & & $\mathrm{PH}_{3}$ & $\mathrm{~S}$ & 2.06 & 0.504 & 0.47 & 0.05 \\
\hline 2012 & Potassium phosphide & & & $\mathrm{PH}_{3}$ & $\mathrm{~S}$ & 2.50 & 0.229 & 0.47 & 0.05 \\
\hline 2013 & Strontium phosphide & & & $\mathrm{PH}_{3}$ & $\mathrm{~S}$ & 2.68 & 0.209 & 0.47 & 0.05 \\
\hline 2308 & Nitrosylsulfuric acid & 00 & 3.3 & $\mathrm{NO}_{2}$ & $\mathrm{~L}$ & 1.890 & 0.724 & 1.00 & 1.0 \\
\hline 2437 & Methylphenyldichlorosilane & 04 & 2 & $\mathrm{HCl}$ & $\mathrm{L}$ & 1.176 & 0.382 & 0.071 & 0.125 \\
\hline 2495 & Iodine pentafluoride & & & $\mathrm{HF}$ & $\mathrm{L}$ & 3.75 & 0.450 & 0.4 & 5 \\
\hline 2691 & Phosphorus pentabromide & $00 / 04$ & 3.2 & $\mathrm{HBr}$ & $\mathrm{S}$ & 2.00 & 0.940 & 0.36 & 2.38 \\
\hline 2692 & Boron tribromide ${ }^{b}$ & 04 & 3.3 & $\mathrm{HBr}$ & $\mathrm{L}$ & 2.65 & 0.971 & 0.19 & 7.36 \\
\hline 2806 & Lithium nitride & 04 & 3.3 & $\mathrm{NH}_{3}$ & S & 1.27 & 0.489 & 0.97 & 6 \\
\hline 2977 & Uranium hexafluoride, fissile & & & $\mathrm{HF}$ & $\mathrm{S}$ & 4.68 & 0.341 & 0.2 & 5 \\
\hline 2978 & Uranium hexafluoride, non-fissile & & & $\mathrm{HF}$ & $\mathrm{S}$ & 4.68 & 0.341 & 0.2 & 5 \\
\hline 2985 & Chlorosilanes, n.o.s. & & & $\mathrm{HCl}$ & $\mathrm{L}$ & 1.10 & 0.565 & 0.43 & 1.01 \\
\hline 2986 & Chlorosilanes, flam., corr, n.o.s. & & & $\mathrm{HCl}$ & $\mathrm{L}$ & 1.10 & 0.565 & 0.43 & 1.01 \\
\hline 2987 & Chlorosilanes, corrosive, n.o.s. & & & $\mathrm{HCl}$ & $\mathrm{L}$ & 1.10 & 0.565 & 0.43 & 1.01 \\
\hline 2988 & Chlorosilanes, wtr-rctv, flam., corr., n.o.s. & & & $\mathrm{HCl}$ & $\mathrm{L}$ & 1.10 & 0.565 & 0.43 & 1.01 \\
\hline 3048 & Aluminum phosphide pesticide & & & $\mathrm{PH}_{3}$ & $\mathrm{~S}$ & 2.40 & 0.587 & 0.47 & 0.05 \\
\hline 3049 & Metal alkyl halides n.o.s. & & & $\mathrm{HCl}$ & $\mathrm{S}$ & 1.60 & 0.500 & 0.05 & 5 \\
\hline 3049 & Metal alkyl halides n.o.s. & & & $\mathrm{HBr}$ & $\mathrm{S}$ & 1.60 & 0.500 & 0.05 & 5 \\
\hline 3052 & Aluminum alkyl halides & & & $\mathrm{HCl}$ & $\mathrm{S}$ & 1.60 & 0.500 & 0.05 & 5 \\
\hline 9191 & Chlorine dioxide, hydrate, frozen & & & $\mathrm{Cl}_{2}$ & $\mathrm{~S}$ & 1.40 & 0.084 & 0.05 & 5 \\
\hline
\end{tabular}

a $\quad E R G=E R G$ edition (2000 or 2004) for which experiment was performed, Type = type of analysis used to derive parameters from experimental data (numbers refer to section and paragraph numbers in Section D.1), St = normal state during shipment (solid, liquid), Dens $=$ density $\left(\mathrm{g} / \mathrm{cm}^{3}\right), \mathrm{S} . \mathrm{Y} .=$ stoichiometric yield of TIH gas $(\mathrm{kg}$ TIH gas $/ \mathrm{kg}$ spilled parent), $\beta=$ efficiency factor (average fraction of S.Y. produced), and $\lambda_{\mathrm{o}}=$ primary rate constant at $20^{\circ} \mathrm{C}$.

b Parent chemical is TIH gas.

c Experiments performed on the closely related compound 4-(chloromethyl)phenyltrichlorosilane were taken to apply to this compound. 
TABLE D.2 Supplementary Information on Water-Reactive Materials in the ERG2004 for 5 Liquid Chlorosilanes Modeled Using an Initial Induction Period Followed by Simple First-order Process ${ }^{a}$

\begin{tabular}{cccccccc}
\hline UN & & & & & & & \\
No. & \multicolumn{1}{c}{ Chemical } & Dens & S.Y. & $\mathrm{m}_{\mathrm{i}}$ & $\beta$ & $\lambda_{\mathrm{o}}\left(\mathrm{min}^{-1}\right)$ & $\begin{array}{c}\mathrm{T}_{\text {ind }} \\
(\mathrm{min})\end{array}$ \\
\hline & & & & & & & \\
1728 & Amyltrichlorosilane & 1.16 & 0.532 & 0.0205 & 0.22 & 0.054 & 5.3 \\
1747 & Butyltrichlorosilane & 1.16 & 0.571 & 0.0233 & 0.26 & 0.099 & 8.6 \\
1763 & Cyclohexyltrichlorosilane & 1.23 & 0.503 & 0.0286 & 0.28 & 0.21 & 4.8 \\
1784 & Hexyltrichlorosilane & 1.0 & 0.496 & 0.0355 & 0.43 & 0.29 & 7.4 \\
1800 & Octadecyltrichlorosilane & 0.984 & 0.281 & 0.0528 & 0.51 & 0.31 & 9.2 \\
\hline
\end{tabular}

a Experiment performed on all materials, Dens $=$ density $\left(\mathrm{g} / \mathrm{cm}^{3}\right), \mathrm{S} . Y .=$ stoichiometric yield of $\mathrm{TIH}$ gas ( $\mathrm{kg}$ TIH gas $/ \mathrm{kg}$ spilled parent), $\mathrm{m}_{\mathrm{i}}=$ the initial production coefficient, $\beta=$ total efficiency factor (average fraction of S.Y. produced), $\lambda_{0}=$ primary rate constant at $20^{\circ} \mathrm{C}$, and $\mathrm{T}_{\text {ind }}=$ initial induction period.

\section{D.3 SUPPLEMENTARY DETAILS IN SUPPORT OF MATERIALS APPEARING IN TABLE D.1}

Most materials appear in Tables D.1 and D.2 and the ERG either because they evolved $\mathrm{TIH}$ gases at reasonable rates in the experiments described in Appendix $\mathrm{C}$ or because they are described as evolving TIH gases in the chemical literature. Reasons for the inclusion of materials in the Table are listed in Sections D.3.1-D.3.3. We also briefly describe why materials were deleted from the list or otherwise not included in Section D.3.4.

\section{D.3.1 Materials that Evolved TIH Gases During Experiments}

The ERG Table contains 40 materials that were shown to evolve gas during the experimental program described in Appendix C. These are listed in Table D.1.

\section{D.3.2 Materials Described as TIHWR in the Literature}

The Table includes 29 materials because of descriptions of their water reactivity in the chemical literature. These are listed in Table D.3. 
TABLE D.3 Chemicals Reported to Be Water-Reactive in the Scientific Literature

\begin{tabular}{|c|c|c|c|}
\hline $\begin{array}{l}\text { UN } \\
\text { No. }\end{array}$ & Name & Comment & Reference \\
\hline 1295 & Trichlorosilane & $\begin{array}{l}\text { Reacts with water and steam to produce heat and toxic } \\
\text { and corrosive fumes (of } \mathrm{HCl} \text { ). }\end{array}$ & Lewis (1993) \\
\hline 1340 & Phosphorus pentasulfide & $\begin{array}{l}\text { Readily liberates toxic hydrogen sulfide and phosphorus } \\
\text { pentoxide and evolves heat on contact with moisture. }\end{array}$ & Lewis (1993) \\
\hline 1397 & Aluminum phosphide & Slowly evolves $\mathrm{PH}_{3}$ in contact with water. & Lewis (1993) \\
\hline 1419 & $\begin{array}{l}\text { Magnesium aluminum } \\
\text { phosphide }\end{array}$ & Evolves $\mathrm{PH}_{3}$ in contact with water. & Lewis (1993) \\
\hline 1432 & Sodium phosphide & Is known to evolve $\mathrm{PH}_{3}$ in contact with water. & Lewis (1993) \\
\hline 1680 & Potassium cyanide & $\begin{array}{l}\text { Included on the basis of its chemical similarity to sodium } \\
\text { cyanide. }\end{array}$ & \\
\hline 1689 & Sodium cyanide & $\begin{array}{l}\text { A spill into a river (on May 20, } 1998 \text { in Kyrgyzstan) led } \\
\text { to evolution of gaseous HCN despite the fact that sodium } \\
\text { cyanide is soluble in water; the evolution of gaseous HCN } \\
\text { is favored by acidic water and by higher temperatures; the } \\
\text { PAD is greater for a spill into water because of the } \\
\text { possibility of such evolution. }\end{array}$ & $\begin{array}{l}\text { Cleven and van } \\
\text { Bruggen }(2000)\end{array}$ \\
\hline 1745 & Bromine pentafluoride & $\begin{array}{l}\text { Explodes on contact with water; products of this rapid } \\
\text { reaction include } \mathrm{HF} \text { and possibly } \mathrm{Br}_{2} \text {. }\end{array}$ & Lewis (1989) \\
\hline 1746 & Bromine trifluoride & $\begin{array}{l}\text { Smokes in air; decomposed violently in water; products of } \\
\text { reaction include } \mathrm{HF} \text { and possibly } \mathrm{Br}_{2} \text {. }\end{array}$ & $\begin{array}{l}\text { Budavari (1996); Lewis } \\
\text { (1989) }\end{array}$ \\
\hline 1777 & Fluorosulfonic acid & $\begin{array}{l}\text { Fumes in moist air; reacts violently with water, although } \\
\text { it is incompletely and reversibly hydrolyzed; products of } \\
\text { reaction include HF. }\end{array}$ & Budavari (1996) \\
\hline 1828 & Sulfur chlorides & $\begin{array}{l}\text { Decomposes when contacted by water to produce heat } \\
\text { and toxic/corrosive fumes. }\end{array}$ & $\begin{array}{l}\text { Carson and Mumford } \\
\text { (1994) }\end{array}$ \\
\hline 1898 & Acetyl iodide & Included by analogy to acetyl chloride. & \\
\hline 1923 & Calcium hydrosulfite & Included by analogy to sodium hydrosulfite. & \\
\hline 1931 & Zinc hydrosulfite & Included by analogy to sodium hydrosulfite. & \\
\hline 2004 & Magnesium diamide & Reacts violently with water, evolving $\mathrm{NH}_{3}$. & Budavari (1996) \\
\hline 2011 & Magnesium phosphide & $\begin{array}{l}\text { Phosphides tend to decompose to } \mathrm{PH}_{3} \text { upon contact with } \\
\text { moisture or acids. }\end{array}$ & Lewis (1989). \\
\hline 2012 & Potassium phosphide & $\begin{array}{l}\text { Phosphides tend to decompose to } \mathrm{PH}_{3} \text { upon contact with } \\
\text { moisture or acids. }\end{array}$ & Lewis (1989). \\
\hline 2013 & Strontium phosphide & $\begin{array}{l}\text { Phosphides tend to decompose to } \mathrm{PH}_{3} \text { upon contact with } \\
\text { moisture or acids. }\end{array}$ & Lewis (1989). \\
\hline 2495 & Iodine pentafluoride & Violent reaction with water; products include HF. & Lewis and Sax (1992) \\
\hline 2977 & $\begin{array}{l}\text { Uranium hexafluoride, } \\
\text { fissile }\end{array}$ & $\begin{array}{l}\text { Is rapidly hydrolyzed by water. Reacts vigorously with } \\
\text { water; products include HF. }\end{array}$ & $\begin{array}{l}\text { Cotton and Wilkinson } \\
\text { (1966); Lewis (1989). }\end{array}$ \\
\hline 2978 & $\begin{array}{l}\text { Uranium hexafluoride, } \\
\text { non-fissile }\end{array}$ & $\begin{array}{l}\text { Is rapidly hydrolyzed by water; reacts vigorously with } \\
\text { water; products include HF. }\end{array}$ & $\begin{array}{l}\text { Cotton and Wilkinson } \\
\text { (1966); Lewis (1989). }\end{array}$ \\
\hline 2985 & Chlorosilanes n.o.s. & $\begin{array}{l}\text { Most chlorosilanes generate } \mathrm{HCl} \text { at some rate on contact } \\
\text { with water. }\end{array}$ & \\
\hline 2986 & $\begin{array}{l}\text { Chlorosilanes, flammable, } \\
\text { corrosive n.o.s. }\end{array}$ & $\begin{array}{l}\text { Most chlorosilanes generate } \mathrm{HCl} \text { at some rate on contact } \\
\text { with water. }\end{array}$ & \\
\hline 2987 & $\begin{array}{l}\text { Chlorosilanes, corrosive } \\
\text { n.o.s. }\end{array}$ & $\begin{array}{l}\text { Most chlorosilanes generate } \mathrm{HCl} \text { at some rate on contact } \\
\text { with water. }\end{array}$ & \\
\hline 2988 & $\begin{array}{l}\text { Chlorosilanes, water } \\
\text { reactive, flammable, } \\
\text { corrosive, n.o.s. }\end{array}$ & $\begin{array}{l}\text { Most chlorosilanes generate } \mathrm{HCl} \text { at some rate if spilled } \\
\text { into water. }\end{array}$ & \\
\hline 3048 & $\begin{array}{l}\text { Aluminum phosphide } \\
\text { pesticide }\end{array}$ & $\begin{array}{l}\text { Aluminum phosphide slowly evolves } \mathrm{PH}_{3} \text { in contact with } \\
\text { water; included despite the fact that the coating applied to } \\
\text { the particles in the pesticide application is likely to slow } \\
\text { the hydrolysis. }\end{array}$ & Lewis (1993) \\
\hline
\end{tabular}


TABLE D.3 (Cont.)

\begin{tabular}{clll}
\hline $\begin{array}{l}\text { UN } \\
\text { No. }\end{array}$ & \multicolumn{1}{c}{ Name } & \multicolumn{1}{c}{ Comment } & Reference \\
\hline 3049 & Metal alkyl halides n.o.s. & $\begin{array}{l}\text { Metal alkyl halides generally react to form hydrogen } \\
\text { halides (HCl, HBr, HI) when mixed with water. }\end{array}$ \\
3052 & Aluminum alkyl halides & $\begin{array}{l}\text { Aluminum alkyl halides generally react to form hydrogen } \\
\text { halides (HCl, HBr, HI) when mixed with water. }\end{array}$ & \\
9191 & $\begin{array}{l}\text { Chlorine dioxide hydrate, } \\
\text { Decomposes in water; products likely to include gaseous } \\
\mathrm{Cl}_{2} .\end{array}$ & Lewis (1989) \\
\hline
\end{tabular}

\section{D.3.3 Materials Included Due to Special Concerns}

Four materials appearing in Table D.1 did not evolve TIH gases during the Appendix C experiments. They were included because they might evolve $\mathrm{TIH}$ gases if spilled under the right circumstances.

1. UN 1384 - sodium hydrosulfite. Experiments found no evolution of gas when the chemical was mixed with room-temperature water, although $\mathrm{SO}_{2}$ and $\mathrm{H}_{2} \mathrm{~S}$ were both detected by their odor. However, this material was retained because of the possibility of substantial evolution of TIH gas from decomposition caused by confined heat of dissolution in restricted amounts of water in spills having the proper geometry (such as a heap on a puddle). Of particular note is the following statement from the EPA/OHSA Joint Chemical Accident Investigation Report on an April 21, 1995 accident at Napp Technologies, Inc., Lodi, NJ: "Sodium hydrosulfite is unstable in the presence of water, heat or humid air, giving off sulfur dioxide gas and other sulfur products in an exothermic reaction. Once initiated, the decomposition process of sodium hydrosulfite supports continued decomposition due to the generation of heat in the exothermic reaction."

2. UN 1541 - acetone cyanohydrin. Experiments found no evolution of gas when the chemical was mixed with room-temperature water. This material was retained on the basis of its chemical similarity to sodium cyanide. A May 20, 1998, spill of sodium cyanide into a river in Kyrgyzstan led to evolution of gaseous HCN (Cleven and van Bruggen 2000).

3. UN 1725 - aluminum bromide (anhydrous). Experiments found no evolution of gas when the chemical was mixed with room-temperature water. It was retained on the basis of its chemical similarity to aluminum chloride.

4. UN 1726 - aluminum chloride (anhydrous). Experiments found no evolution of gas when the chemical was mixed with room-temperature water. It was retained because the small scale of the experiment might have masked its TIHWR character. Aluminum chloride is cited as generating $\mathrm{HCl}$ in contact 
with water: "Reacts with air moisture to form corrosive $\mathrm{HCl}$ gas. Violent reaction when a stream of water hits a large amount" (Carson and Mumford 1994).

\section{D.3.4 Materials No Longer Recommended as TIHWR Materials}

Eleven materials previously recommended by ANL and the University of Illinois at Chicago as TIWHR materials were removed from the current list. (Note that not all these materials actually appeared in the ERG2000).

1. UN 1433 - stannic phosphide. No evolution of TIH gas was observed in the experiments (see Appendix C).

2. UN 1714 - zinc phosphide. No evolution of TIH gas was observed in the experiments (see Appendix C).

3. UN 1736 - benzoyl chloride. No evolution of TIH gas was observed in the experiments (see Appendix C). However, literature sources do mention TIH gas evolution, for example: "Reacts strongly with water or water vapor, producing heat and toxic, corrosive fumes" (Carson and Mumford 1994).

4. UN 1808 - phosphorus tribromide (solid). This compound reacted with water, but no gaseous $\mathrm{HBr}$ was evolved when excess water was present; the excess water apparently dissolved the $\mathrm{HBr}$ as rapidly as it was formed

5. UN 1939 - phosphorus oxybromide (solid). Reacted with water, similarly to phosphorus tribromide; the excess water apparently dissolved the $\mathrm{HBr}$ as rapidly as it was formed.

6. UN 1749 - chlorine trifluoride. This material reacts explosively with water to generate $\mathrm{HF}$ and possibly $\mathrm{Cl}_{2}$. The compound is gaseous above $11.8^{\circ} \mathrm{C}$ and was therefore treated as a TIH gas in its own right.

7. UN 1807 - phosphorus pentoxide. This material reacts explosively with water to generate water-soluble phosphoric acid. Previously included by analogy to $\mathrm{SO}_{3}$ because of the possibility that this very rapid, exothermic reaction might raise a toxic acidic mist in a spill. Since phosphoric acid is not a TIH material, DOT chose not to include phosphorus pentoxide.

8. UN 1831 - oleum. Oleum is a solution of sulfur trioxide in sulfuric acid. It fumes strongly in moist air (Lewis 1993) and reacts with water and water vapor to form sulfuric acid mists (NIOSH Substance Profile; see http://ntp.niehs.nih.gov/ntp/roc/eleventh/ profiles/s164sulf.pdf). The reaction is quite exothermic. However, oleum is already a TIH material and spills into 
water would likely not lead to PADs in excess of those already listed for land based spills.

9. UN 1829 - sulfur trioxide. Like oleum, this compound reacts with water and water vapor to form sulfuric acid mists (NIOSH Substance Profile). Excluded for the same reasons as oleum.

10. UN 2442 - trichloroacetyl chloride. This compound was reacted with water in the experiments, but no evolution of gaseous $\mathrm{HCl}$ was observed (see Appendix C).

11. Sodium methylcarbamodithioate (metam sodium) (no UN number). This material has a known history of water reactivity but is excluded because it does not have a $\mathrm{UN}$ number. 
